TM-1505

\title{
Fermilab D-0 Experimental Facility Energy Conservation Report and Mechanical Systems Design Optimization and Cost Analysis Study
}

\author{
S. F. Krstulovich \\ Fermilab Construction Engineering Services \\ Fermi National Accelerator Laboratory \\ P.O. Box 500, Batavia, Illinois 60510
}

October 31, 1987 


\section{FEFMILAE D-O EXFEFIMENTAL FACILITY ENERGY CONSEFUATION FEFOFT AND}

MECHANICAL SYSTEMS DESIGN OFTIMIZATION AND OOST ANALYSIS STUDY

21 DCTDEEF, 1987

S.F.FFSTULOUICH

FEFMLAE CONSTEUCTION ENGINEEFING SEFUTCES 


\section{FERMILAB D-0 EXPERIMENTAL FACILITY}

\section{Energy Conservation Report and}

Mechanical Systems Design Optimization and Cost Analysis Study

S. F. Krstulovich

Fermi National Accelerator Laboratory

P.O. Box 500

Batavia, IL 60510

\section{TABLE OF CONTENTS}

Title

$\underline{\text { Page }}$

I. ANALYSIS DATA SUMMARY

II. SITE WEATHER DATA AND DESIGN PROFILES

II-1

III. SITE MAIN RING LAKE COOLING DATA

III-1

IV. SUBCONSTRUCTION 1/3-COLLISION AND ASSEMBLY HALLS SYSTEMS ANALYSIS
a. Building Sector Physical Parameters
IV-1
b. Building Load Calculations
IV-3
c. System Comparison Performance Simulations
IV-7
1. Design Basis System (Reciprocating
IV-8
Chillers with Energy Features)
2. Alternate System No. 1 (Centrifugal
IV-24
3. Alternate System No. 2 (FY1975
IV -40 Reciprocating Chillers - Basic System) 
V. SUBCONSTRUCTION 2/3-MCH, PLATFORM \& SIDE BAY AREAS WITH DCHW SYSTEMS ANALYSIS

a. Building Sector Physical Parameters V-1

b. Building Load Calculations V-3

$\begin{array}{ll}\text { c. System Comparison Performance Simulations } & \text { V-7 }\end{array}$

1. Design Basis System (Reciprocating Chillers V-8 with Energy Features)

2. Alternate System No. 1 (Centrifugal Chillers V-22 with Energy Features)

3. Alternate System No. 2 (FY1975 Reciprocating V-36 Chillers - Basic System)

VI. SUBCONSTRUCTION 3/3-LCW COOLING SYSTEMS ANALYSIS

a. Building Sector Physical Parameters VI-1

b. Building Load Calculations VI-3

c. System Comparison Performance Simulations VI-5

1. Design Basis System (Lake Water Cooling VI-6 with Energy Features)

2. Alternate System No. 1 (Pond Water VI-15 Cooling - Basic System)

VII. SYSTEMS LIFE CYCLE COST ANALYSIS AND PAYBACK CALCULATIONS
a. LCC Analysis for Design Basis System
VII-1
b. LCC Analysis for Alternate System No. 1
VII-5
c. LCC Analysis for FY1975 Alternate System No. 2 VII-9
d. LCC Analysis Present Worth Comparisons VII-13
e. Payback Analysis
VII-16 
$\underline{I}$

mom

किनि कालाला

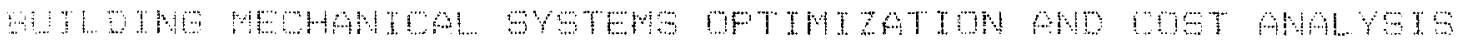

31 OMTOBEF, 59

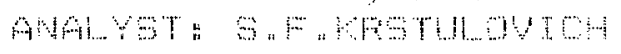

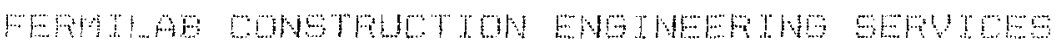




\section{ENERGY CONSERVATION REPORT}

Analysis Data Summary

SCOPE: This report is developed as part of the Fermilab D-0 Experimental Facility Project Title II Design Documentation Update under the provisions of D.O.E. Document 6430.1, Chapter XIII-21, Section 14, paragraph a. As such, it concentrates primarily on HVAC mechanical systems design optimization and cost analysis.

a. Identification of Methods used for Building Energy Consumption Analysis: Carrier Corporation Model E20-II software run on an IBM PS/2 computer has been used to generate all loads, weather calculations, system simulations, life cycle costs, and payback data. This program has been used in conjunction with published data from:

1. ASHRAE Fundamentals - Chapter 24: Weather data and design considerations

2. Fermilab On-Site Records of lake and cooling pond performance

3. Air Force Manual 88-29: Facility design and planning engineering weather data

4. Richardson Engineering Services Corporation: Construction Costs Data

b. Methodology of Analysis: Life cycle cost analysis employs the unified present worth approach. Significant figures used in this exercise are 20 years reflecting minimum useful system life and $7.0 \%$ discount factor based upon projected outlooks from Chase, Data Resource Institute, Mellon and Evans. Project construction costs are paid off as a lump sum in the first year without amortization. The electrical energy escalation costs, used in consideration of the same projected outlooks used for the discount factor, seem rather conservative in the light of Illinois Edison's particular problems with over-built nuclear facilities coming on line.

Payback analysis is based on the simple payback method with a maximum two (2) year return on investment period targeted.

Building energy analysis is based on the bin method with temperature/humidity projections made by algorithm from the ASHRAE design weather data. System performance is executed in an integrated dynamic fashion from selected points of rating. 
To analyze a complex structure, like this facility, the total building was divided into three (3) Subconstructions based upon the entirely different design conditions and systems employed in each of these zones. In essence, each of these subconstructions is analyzed as a separate building except insofar as they affect one another at their thermodynamic boundaries. The three (3) basic subconstructions are as follows:

Subcon 1/3: Describes the Collision and Assembly Halls

Subcon 2/3: Describes the Movable Counting House, Detector Platform and Side Bay Areas, together with the Deionized Chilled Water Systems

Subcon 3/3: Describes the Low Conductivity Cooling Water Systems

Applicable alternative mechanical systems were selected for each of the Subconstructions and were then combined as total building combined systems into several possible arrangements. These were then compared to each other, including the designed combined systems arrangement and FY1975 combined systems arrangement, to evaluate their merits.

For Subconstruction $1 / 3$ (the Collision and Assembly Halls), three (3) alternative HVAC systems were evaluated, including a typical FY1975 design. The systems were:

1. Reciprocating chillers with lake water cooling, chilled water free cooling heat exchanger, pump cycling, high bay heat circulating fans, lake water filtration, integrated DDC controls, high efficiency motors, central station air handling units with nested fan inlet vanes and integrated enthalpy economizer. This represents our as designed system.

2. Centrifugal chillers with all other energy features incorporated in the systems arrangement above. This represents Alternate System No. 1.

3. Reciprocating chillers with pond water cooling and none of the energy features incorporated in the systems arrangements above. This represents Alternate System No. 2, our FY1975 basic system design.

For Subconstruction 2/3 (the Movable Counting House, Detector Platform and Side Bay Areas, together with the Deionized Chilled Water Systems), three (3) alternative HVAC systems were evaluated including a typical FY1975 design. The systems were: 
1. Reciprocating chillers with lake water cooling, central station air handling units, chilled water computer room air conditioning units, water cooled electronics racks and these energy features: chilled water free cooling heat exchanger, pump cycling, lake water filtration, integrated DDC controls, high efficiency motors, integrated enthalpy economizer and supply air cooling temperature reset. This represents our as designed system.

2. Centrifugal chillers with lake water cooling, central station air handling units, chilled water computer room air conditioning units, water cooled electronics racks and all of the energy features incorporated in the systems arrangement above. This represents Alternate System No. 1.

3. Reciprocating chillers with pond water cooling, central station air handling units, chilled water computer room air conditioning units, water cooled electronics racks and none of the energy features incorporated in the systems arrangement above. This represents Alternate System No. 2, our FY1975 basic system design.

For Subconstruction $3 / 3$ (the Low Conductivity Cooling Water Systems), two (2) alternative HVAC systems were evaluated, including a typical FY1975 design. The systems were:

1. Hydronic free cooling heat exchanger with these energy features: lake water cooling, lake water filtration, high efficiency motors and automatic lake water pump staging. This represents the $3 / 3$ configuration for both our as designed system and the Alternate System No. 1.

2. Hydronic free cooling heat exchanger with pond water cooling and none of the energy features incorporated in the systems arrangement above. This represents Alternative System No. 2, our FY1975 basic system design.

All of these alternative HVAC systems for each Subconstruction area were then combined to yield three (3) total building combined systems arrangements for comparison. These arrangements are as follows:

1. The as designed arrangement (consisting of the first alternative from each Subconstruction), which is a reciprocating chiller system with lake water cooling and full building energy conservation features.

2. Alternate Arrangement No. 1 (consisting of the second alternative from Subconstructions $1 / 3$ and $2 / 3$ combined with the first alternative from Subconstruction $3 / 3$ ), which is a centrifugal chiller system with lake water cooling and full building energy conservation features. 
3. Alternate Arrangement No. 2 - our base case FY1975 design (consisting of the third alternative from Subconstructions $1 / 3$ and $2 / 3$ combined with the second alternative from Subconstruction $3 / 3$, which is a reciprocating chiller system with pond water cooling and none of the energy conservation features of the arrangements above.

The systems arrangements used in this analysis as our FY1975 base case are actually typical of designs used as late as the early 1980s and, therefore, are probably more favorable than an actual 1975 design would be. This, however, only acts to make the calculated savings advantage of the designed systems more conservative. The advantage to using these arrangements as our base case is that we can use actual experience in the operation of such facilities for a more realistic comparison to the designed systems arrangement. This experience factor is especially valuable to analysis accuracy since it is impossible to find a generic example typical of such specialized nuclear research facilities from the $1970 \mathrm{~s}$, with complex building pressurization profiles, massive detector load cycling, and so forth.

In adapting simulation profiles to standard software format options, several modeling approximation techniques were employed. Fermilab lake water for cooling is seen from historical data to be sensitive to weather conditions, and is therefore modeled after open cooling towers rather than stable groundwater sources. The Main Ring cooling ponds are also weather sensitive according to historical data, but maintain higher temperatures than lake water due to Main Ring cooling loads, and are therefore modeled after closed circuit cooling towers. The low conductivity water systems (Subconstruction 3/3) were modeled as a one square foot perimeter area with no exterior surface boundaries, no load other than the LCW cooling wattages, and as having "cooling only" requirements. Due to software constraints, the LCW system equipment is fictionally configured as a constant volume central air handler with virtually no air side static pressure drop, coupled to a free cooling heat exchanger and pumping system. This effectively eliminates fictional fan energy from the equation and permits us to simulate all of the other system features. The effect of nested air handling unit inlet vanes is modeled through a corresponding adjustment in fan static pressure requirements. The staging of pumps is likewise modeled by an adjustment in pump head requirements corresponding to the facility load cycling profiles. Both integrated DDC building pressurization control and DDC calculated outside air intake control are modeled by an adjustment to the average outside air volume corresponding to historical operating data from a similar facility on site. Lake water filtration and high efficiency motors are both modeled by a corresponding adjustment in fan, pump and chiller efficiency. Finally, high bay area ceiling heat circulation fans are modeled by an adjustment to the area heating set point corresponding to lowered temperature averages due to decreased area stratification. 
A major question in the design of the D-0 facility mechanical; systems was the selection of reciprocating versus centrifugal chillers. Apart from the danger of driving the centrifugal units into a surge condition during periods of abnormally high lake water temperatures and the need for higher condenser water flows to lower LWT, it was decided to favor the centrifugal over reciprocating units in this analysis so as to place beyond question any benefits of reciprocating units. To this end, it was assumed that the construction costs of both types of units would be the same, even though the centrifugal units would actually cost about $\$ 20,000$ more than the reciprocating type.

c. Description of the Major Energy Conservation Features Selected: The design building HVAC systems arrangement constitutes a central reciprocating chiller plant with filtered lake water cooling, computerized Direct Digital Controls (DDC) and full building energy conservation features. Due to the strict process requirements of this facility, advances in energy conservation are primarily focused on refinement of necessary system types rather than in a comparison of optional system varieties.

The selection of reciprocating versus centrifugal chillers is borne out in the analysis by the fact that although centrifugal units have a higher rated efficiency at full load than reciprocating units, the wide swings in load cycling coupled with prolonged periods of chilled water free cooling put our chillers at modest to very low load during most of the year. At this point the curves for reciprocating chillers outperform the centrifugals significantly, such that there is actually an overall annual energy savings for the reciprocating units. Another feature along these lines is that the centrifugal units would require higher condenser water flows at times to prevent surging, and this in turn shows up as increased energy costs for pumping over the reciprocating units selection. The reciprocating chillers are of the high efficiency, multi-staged type with dual circuited condensers for increased efficiency at part load, and are augmented by an integrated hydronic economizer free cooling heat exchanger using lake water to permit full or partial chiller shut-down for several months out of the year. Chiller efficiency as well as low conductivity and chilled water free cooling heat exchanger approach is improved by use of lake water high efficiency filtration which reduces both heat transfer fouling factors as well as heat exchanger maintenance costs.

The use of lake water over Main Ring cooling pond water is also a significant energy saving factor. First of all, the lower temperatures and annual ice-pack of this large body of water, which is not presently being used for any other cooling purposes, permits chillers to operate at greater efficiencies and allows for longer periods of free cooling at lower temperatures than would be obtainable from the Main Ring cooling ponds. Secondly, however, by not using the Main Ring cooling ponds, we keep them from increasing in temperature which would adversely affect the efficiency of the major Main Ring 
cooling systems. The energy cost avoidance in this case would be considerable, but is not credited in this analysis for the sake of simplicity. Along these same lines, reduced LCW cooling water temperatures obtainable in summer using lake water will increase equipment efficiencies at the D-0 facility, which is also not credited in this analysis for the sake of simplicity. The operation of lake water pumps is also automatically staged by computer through a reading of supply and return water temperatures to match cooling water load requirements and thus allow for lake water pumping costs energy savings. The incremental nature of load staging at this facility favored the use of simple, low-cost pump staging techniques over variable speed designs in life cycle cost analysis.

Both fan and pump performance in every case is enhanced through the use of high efficiency motors, which contribute significantly to energy savings in the light of the 24 hour operation of these units at the D-0 site. All fans, pumps and air handling units at this site are of the high quality, industrial grade type, selected specifically for high efficiency performance. Air handling units are equipped with nested inlet vanes to increase fan efficiency, in addition to integrated enthalpy economizer systems for free air-side cooling capacity.

The Assembly Hall High Bay Area is equipped with ceiling mounted heat circulating fans to reduce air temperature stratification and thus conserve energy by reducing required indoor air temperature at the roof level to maintain temperature setpoint at the floor. This in turn reduces building heat transmission losses by reducing the average effective temperature difference across building surfaces exposed to the outdoors.

Air handling systems in the Side Bay areas are designed to further conserve energy by computerized supply air temperature reset which looks at all zone temperatures and recalculates optimum AHU supply air temperatures so as to delay turn-on of any individual zone heating coil for as long as possible before temperature in any other zone rises above the comfort tolerance level. These savings are not credited in this analysis for the sake of simplicity. Along these same lines, dehumidification reheat in the Assembly and Collision Halls is reset in a similar manner by computer to minimize heating coil turn-on, which is also not credited in this analysis for the sake of simplicity.

System performance is further enhanced by the use of dynamic control through a Direct Digital Control system employing proportional-integral-derivative response coupled to pulse width modulation for permanent calibration - high accuracy tracking. This technology also allows for sophisticated strategy through its boolean operatives. The system is of a fully modular and distributed processing architecture which is expandable by adding to each subsystem as required. The DDC controls system consists of standalone intelligent local field processors for all building systems 
connected to one another and to a local front end computer to create a local area network. The system is modular both in design of software as well as hardware and shall allow for change of function and operation in the field without programming changes or changes to hardware. Control strategies and setpoints may be changed on line by Fermilab personnel to the end that integral energy management features (such as demand limiting) may be brought on line as historical data is generated. Energy conservation through high accuracy control tuning which eliminates the constant setpoint over-shooting associated with conventional control systems is also not credited in this analysis for the sake of simplicity.

Two significant energy conservation features deriving directly from the use of DDC computerized controls at the D-0 facility serve to reduce excessive intake of outdoor air by HVAC systems. The first feature constantly calculates and resets outdoor air intake dampers to maintain exact minimum percentage of outdoor air based on mathematical comparison of outdoor air, return air and mixed air temperatures. This eliminates excessive outdoor air intake during cold weather due to increased outdoor air density, which is typical of conventional control systems designed to merely maintain preset minimum outdoor air damper position.

The second feature allows the central control computer to simultaneously look at the many building pressurization zones, with their varying degrees of positive, negative and neutral relationships to outdoors and to orchestrate the total building $\mathrm{HVAC}$ system, so as to preclude individual control systems from fighting each other and gulping or exhausting excessive outdoor air while maintaining fine pressure control. Our experience with conventional controls in similar applications proves that this is impossible without DDC, and results in periods of uncontrollable cycling with subsequent energy waste beyond the conservative factors employed in this analysis.

d. Evaluation of Solar Applications: The overwhelming balance of excess heat in this structure precludes the use of either active or passive solar energy applications.

e. Discussion of Evaluation of Non-Renewable Energy Supply Alternatives: Due to the electrically intensive nature of this facility, coupled with the abundance of waste heat, the use of natural gas (the only other viable non-renewable energy supply on site) is precluded over electricity.

In engineering the overall integrated mechanical systems configuration, consideration has been made to assure minimal utilization of non-renewable resources in the form of electrical energy with respect to D.O.E. Document 4330.3 . 
f. Estimates of Total HVAC Energy Input to the Building: The Fermilab D-0 Experimental Facility is characterized by exceptionally intense cooling loads. Overall building design load intensity is calculated at 100 square feet per ton.

Building Annual Energy Input for Designed HVAC Systems:

KWH Per Year: $1,182,331$

Annual DOE Resource Impact Factor: 4,035,910 MBH

Annual BTU/Gross Square Foot/Year: 95,638

g. $45 \%$ Energy Use Reduction Goal Data:

1. The following information represents the estimated total HVAC energy use of a representative building of the appropriate category in FY1975:

Building Annual Energy Input for FY1975 HVAC Systems:

KWH Per Year: $2,128,445$

Annual DOE Resource Impact Factor: 7,264,383 $\mathrm{MBH}$

Annual BTU/Gross Square Foot/Year: 172,142

2. The following is the estimated percentage of energy use reduction for the designed HVAC systems as opposed to typical FY1975 HVAC Systems:

Building Present Scope: $45 \%$ Reduction

As described within the text, this goal is realized on the basis of conservative analysis, with several energy conservation features not being credited for the sake of analysis simplicity.

3. Due to the atypical requirements, loads, and design of this building, the method of simulating by computer the performance of typical FY1975 HVAC systems in the as-designed structure has been used to estimate the baseline HVAC energy use of a representative building of the appropriate category in FY1975.

4. The estimated additional HVAC systems construction cost to achieve the $45 \%$ percent of energy use reduction over the typical FY1975 systems is as follows:

Building Present Scope: $\$ 77,500$ additional costs (10\%)

Most of the additional costs derive from extension of cooling water lines to the Main Ring lake in lieu of the Main Ring cooling ponds, but are not fully credited in energy cost avoidance to operation of the Main Ring systems as discussed above. Furthermore, these additional costs do not take into account actual construction cost savings from using the designed DDC control systems in lieu of conventional controls. Actual 
comparison of controls costs for D-0 with those for similar facilities on site suggest savings of as much as $\$ 50,000$, which would reduce actual additional costs to about $\$ 27,500(4 \%)$. In view of the complexity of calculating exact control cost differences however, it was decided to ignore such savings for the sake of simplicity in this analysis.

5. The estimated energy cost savings over the minimal 20-year projected HVAC systems life including additional HVAC systems construction cost, first year energy cost savings, and present worth energy source factors are as follows:

Building Present Scope: $\$ 969,807$ cost savings

h. Outline of Metering Provisions: Due to the nature of DDC control systems and the need for metering data acquisition to execute the energy conservation strategies, virtually all major energy flows are intrinsically monitored, summarized, and printed at the local front end computer printer. This information will also be useful in generating site weather data, evaluating lake cooling water performance data (especially as it is related to weather), give Fermilab personnel data to evaluate alterations to initial energy management strategies and confirm HVAC systems performance in relation to estimated projections. Among the items metered are:

1. All lake cooling water flows.

2. All energy BTU's wasted from building through both cooling water and exhaust air.

3. All hours of free cooling for individual systems.

4. All compressor run time hours for chillers.

5. All heater stages run time hours.

6. All general equipment status data.

j. Comparison of Design HVAC System, Alternate Centrifugal Chiller System, and FY1975 Base Case System: Results of these comparisons are summarized on the three (3) Systems Summary Sheets attached hereafter. 
TOTAL BUILDING SYSTEMS COMBINED PERFORMANCE SUMMARY

DESIGNED CONFIGURATION

RECIPROCATING CHILLERS - FULL BUILDING ENERGY FEATURES

Statistic

Present Scope

HVAC Systems Parameters

Area:

Tons:

SF/Ton:

Annual Operating Cost:

Annual Op. Cost/SF:

Annual Maintenance Cost:

Construction Cost:

$42,200 \mathrm{SF}$

420

100

$\$ 59,125$

$\$ \quad 1.40$

$\$ 12,000$

$\$ 752,900$

20 Yr. Cumulative Cost:

HVAC Energy

$\mathrm{KWH} / \mathrm{Yr}$.

DOE RUF MBH:

DOE RIF MBH:

$\mathrm{BTU} / \mathrm{SF} / \mathrm{Yr}$.

Energy Reduction from 1975:

Non-HVAC Energy

$\mathrm{KWH} / \mathrm{Yr}$.

DOE RUF MBH:

DOE RIF MBH:

$\mathrm{BTU} / \mathrm{SF} / \mathrm{Yr}$.

$$
\begin{array}{r}
1,182,331 \\
13,717,127 \\
4,035,910 \\
95,638 \\
45 \%
\end{array}
$$

\section{Total Building Energy}

$\mathrm{KWH} / \mathrm{Yr}$.

DOE RUF MBH:

DOE RIF MBH:

$\mathrm{BTU} / \mathrm{SF} / \mathrm{Yr}$.
$7,686,513$

$89,163,546$

$26,234,068$

621,660
Report Page Number Statistic References

$(\mathrm{IV}-11)+(\mathrm{V}-11)+(\mathrm{VI}-9)$

(VII-2)

(VII-2)

(VII-4)

$(\mathrm{IV}-11)+(\mathrm{V}-11)+(\mathrm{VI}-9)$

$(\mathrm{IV}-11)+(\mathrm{V}-11)+(\mathrm{VI}-9)$

$(\mathrm{IV}-11)+(\mathrm{V}-11)+(\mathrm{VI}-9)$

$(\mathrm{IV}-11)+(\mathrm{V}-11)+(\mathrm{VI}-9)$

IV-11) +(V-11)+(VI-9)

(IV-11) $+(\mathrm{V}-11)+(\mathrm{VI}-9)$ 
TOTAL BUILDING SYSTEMS COMBINED PERFORMANCE SUMMARY

ALTERNATE NO. 1 CONFIGURATION

CENTRIFUGAL CHILLERS - FULL BUILDING ENERGY FEATURES

Statistic

Present Scope

HVAC Systems Parameters

Area:

Tons:

SF/Ton:

Annual Operating Cost:

Annual Op. Cost/SF:

Annual Maintenance Cost:

Construction Cost:

$\%$ of Design Constr. Cost:

Constr. Cost Difference:

Annual Op. Cost Diff.:

Design System Payback:

20 Yr. Cumulative Cost:

Cumulative Cost Diff.:

HVAC Energy

$\mathrm{KWH} / \mathrm{Yr}$.

DOE RUF MBH:

DOE RIF MBH:

$\mathrm{BTU} / \mathrm{SF} / \mathrm{Yr}$.

Energy Increase

from Design:

Energy Reduction

from 1975:

Non-HVAC Energy

$\mathrm{KWH} / \mathrm{Yr}$.

DOE RUF MBH:

DOE RIF MBH:

$\mathrm{BTU} / \mathrm{SF} / \mathrm{Yr}$.

$\underline{\text { Total Building Energy }}$

$\mathrm{KWH} / \mathrm{Yr}$.

DOE RUF MBH:

DOE RIF MBH:

$\mathrm{BTU} / \mathrm{SF} / \mathrm{Yr}$.
Report Page Number Statistic References

$$
\begin{aligned}
& 42,200 \mathrm{SF} \\
& 420 \\
& 100 \\
& \begin{array}{rr}
\$ & 65,087 \\
\$ & 1.54 \\
\$ & 12,000 \\
\$ & 752,900 \\
& 100 \%
\end{array} \\
& \begin{array}{rr}
\$ & 0.0 \\
\$ & 5,962
\end{array} \\
& 0.0 \text { years } \\
& \$ 2,431,364 \\
& \$ \quad 91,217 \\
& \begin{array}{l}
\left.(\mathrm{IV}-2)+\left(\begin{array}{l}
\mathrm{V}-2)+(\mathrm{VI}-2) \\
(\mathrm{IV}-4)
\end{array}\right)+(\mathrm{V}-4)+\mathrm{VI}-4\right)
\end{array} \\
& (\mathrm{IV}-27)+(\mathrm{V}-25)+(\mathrm{VI}-9) \\
& \text { (VII-6) } \\
& \text { (VII-6) } \\
& \left\{\begin{array}{l}
\text { VII-6)-(VII-2) } \\
(\text { IV-27) }+(\text { V-25 })+(\text { VI-9) })- \\
(\text { IV-11 })+(\text { V-11 })+(\text { VI-9) } \\
\text { VII-17) }
\end{array}\right.
\end{aligned}
$$

$$
\begin{array}{r}
7,686,513 \\
89,163,546 \\
26,234,068 \\
621,660
\end{array}
$$

$$
\begin{array}{r}
1,261,817 \\
14,639,178 \\
4,307,200 \\
102,066
\end{array}
$$$$
107 \%
$$$$
41 \%
$$

$$
\begin{aligned}
& (\mathrm{IV}-27)+(\mathrm{V}-25)+(\mathrm{VI}-9) \\
& (\mathrm{IV}-27)+(\mathrm{V}-25)+(\mathrm{VI}-9) \\
& (\mathrm{IV}-27)+(\mathrm{V}-25)+(\mathrm{VI}-9)
\end{aligned}
$$


TOTAL BUILDING SYSTEMS COMBINED PERFORMANCE SUMMARY

ALTERNATE NO. 2 FY1975 CONFIGURATION

RECIPROCATING CHILLERS - BASIC BUILDING DESIGN

Statistic

Present Scope

HVAC Systems Parameters

Area:

Tons:

SF/Ton:

Annual Operating Cost:

Annual Op. Cost/SF:

Annual Maintenance Cost:

Construction Cost:

$\%$ of Design Constr. Cost:

Constr. Cost Difference:

Annual Op. Cost Diff.:

Design System Payback:

20 Yr. Cumulative Cost:

Cumulative Cost Diff.:

HVAC Energy

$\mathrm{KWH} / \mathrm{Yr}$.

DOE RUF MBH:

DOE RIF MBH:

$\mathrm{BTU} / \mathrm{SF} / \mathrm{Yr}$.

Energy Increase

from Design:

Non-HVAC Energy

$\mathrm{KWH} / \mathrm{Yr}$.

DOE RUF MBH:

DOE RIF MBH:

$\mathrm{BTU} / \mathrm{SF} / \mathrm{Yr}$.

$$
\begin{array}{r}
2,128,445 \\
24,689,962 \\
7,264,383 \\
172,142
\end{array}
$$

$180 \%$

$$
\begin{array}{r}
7,686,513 \\
89,163,546 \\
26,234,068 \\
621,660
\end{array}
$$

Report Page Number Statistic References
$(\mathrm{IV}-2)+(\mathrm{V}-2)+(\mathrm{VI}-2)$

$(\mathrm{IV}-4)+(\mathrm{V}-4)+(\mathrm{VI}-4)$

420

100

$\$ 106,423$

2.52

$\$ 10,000$

$\$ 675,400$

$90 \%$

$-\$ 77,500$

$\$ 47,298$

1.7 years

$\$ 3,309,954$

$\$ 969,807$

(VII-12)

\section{Total Building Energy}

KWH/Yr.

DOE RUF MBH:

DOE RIF MBH:

BTU/SF/Yr.
(VII-15)

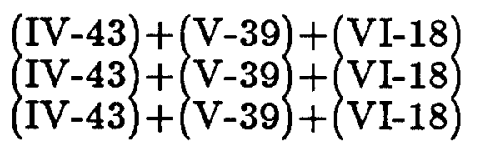

(VII-10)

(VII-10)

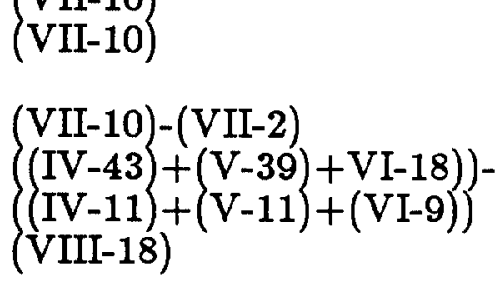

$(\mathrm{IV}-43)+(\mathrm{V}-39)+(\mathrm{VI}-18)$

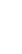




\section{APPENDICES}

At the authors' request, the Appendices to TM-1505 are being distributed upon request only.

If you would like a copy of same, please contact the Publications Office at MS \#107 or Ext. 3278. 
TM-1505

\title{
Appendices
}

\section{Fermilab D-0 Experimental Facility Energy Conservation Report and Mechanical Systems Design Optimization and Cost Analysis Study}

\author{
S. F. Krstulovich \\ Fermilab Construction Engineering Services \\ Fermi National Accelerator Laboratory \\ P.O. Box 500, Batavia, Illinois 60510
}

October 31,1987 
FERMILAB D-O EXFEFIMENTAL FACILITY ENERGY CONSEFVATION REFORT

AND

MECHANICAL SYSTEMS DESIGN OFTIMIZATION AND COST ANALYSIS STUDY

31 OCTOBER, 1987

S.F.KRSTULOVICH

FEFMILAB CONSTRUCTION ENGINEERING SERVICES 
II.

\section{FEFMILAE DOO EXFEFIMENTAL FACILITY \\ SITE WEATHEF DATA AND DESIGN FRUFILES}

उ1 OCTOEEF, 1987

ANALYST: S.F.FESTULOVICH

FEFMTLAE CONSTFUCTION ENGINEERING SEFUICES 
Site Name : AUROFA, IL FEFMI, User Defined

Date : $11-03-87$

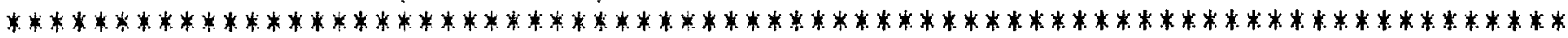

TAELE 1. SITE DESIGN WEATHEF: DATA

Latitude $=42$ dea.

Suminer design dry bulb $=9 . \mathrm{F}$

Summer desian wet bulb $=76 \mathrm{~F}$

Elevation

$=744$ Feet

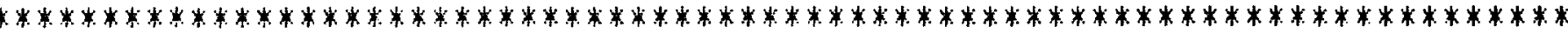

TAELE 2. FEAY SOLAF: GAINS (Etu/ hr- $5 q f t$ ))

\begin{tabular}{|c|c|c|c|c|c|c|c|c|c|c|}
\hline I Month & ! & $N$ & $N E$ & $E$ & $S E$ & 5 & SW & $w$ & NW & $H$ \\
\hline $\begin{array}{l}\text { January } \\
\text { July }\end{array}$ & i & $\begin{array}{r}5.6 \\
17.1\end{array}$ & $\begin{array}{r}13.2 \\
144.7\end{array}$ & $\begin{array}{l}107.4 \\
189.6\end{array}$ & $\begin{array}{l}175.8 \\
148.8\end{array}$ & $\begin{array}{r}189.1 \\
88.4\end{array}$ & $\begin{array}{l}175.8 \\
148.8\end{array}$ & $\begin{array}{l}107.4 \\
189.6\end{array}$ & $\begin{array}{r}13.2 \\
144.7\end{array}$ & $\begin{array}{l}107.6 \\
264.5\end{array}$ \\
\hline
\end{tabular}

Carrier peat: solar data used.

Values corrected for altitude ( $744 \mathrm{ft}$ ), design dewpoint ( $69.3 \mathrm{~F}$ ) \% Available Sunshine : Average data: Summer $=73 \%$; winter $=35 \%$

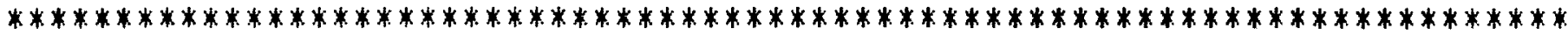
TABLE $\Xi$. DCCUFANCY SCHEDULE DATA

\begin{tabular}{|c|c|c|c|c|c|c|c|c|c|c|c|c|c|c|}
\hline$i$ & $i$ & Sun & $i$ & Mon & i & Tule & $i$ & Wed & : & Thu & $i$ & Fri & $i$ & Sat \\
\hline Eegin & : & OFF & : & $7 \mathrm{~A}$ & ! & $7 \mathrm{~A}$ & i & $7 \mathrm{~A}$ & i & $7 A$ & : & $7 \mathrm{~A}$ & i & $\Rightarrow A$ \\
\hline End & 1 & DFF & 1 & $\Leftrightarrow F$ & 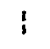 & $B F$ & $i$ & $\Leftrightarrow F$ & $!$ & $6 F$ & i & $\therefore F$ & $!$ & $2 \mathrm{~F}$ \\
\hline
\end{tabular}

Average occupancy : 10.0 hrs/day 6.0 days/weel:

Number of summer shutdown days $=0$

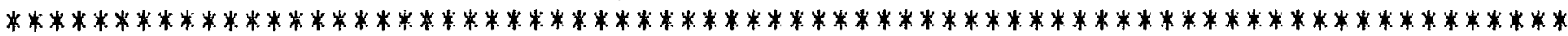
TAELE 4. TEMFEFIATLIFE EIN DATA

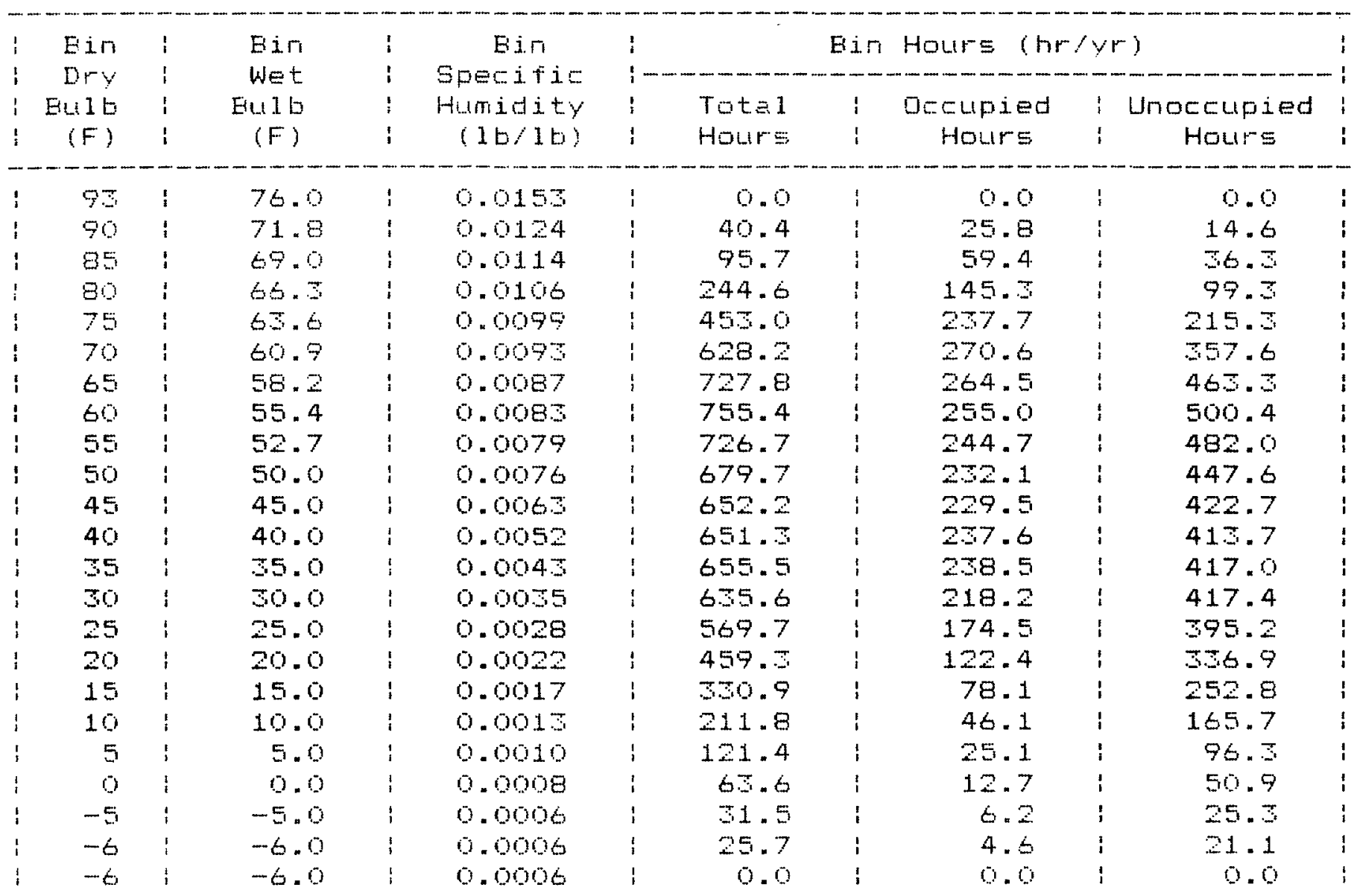


BIN HOUR PROFILE

Site : AURORA IL FERMI, User Def ined Summer Dry Bulb $=93 \mathrm{~F}$

Date : 11-03-87

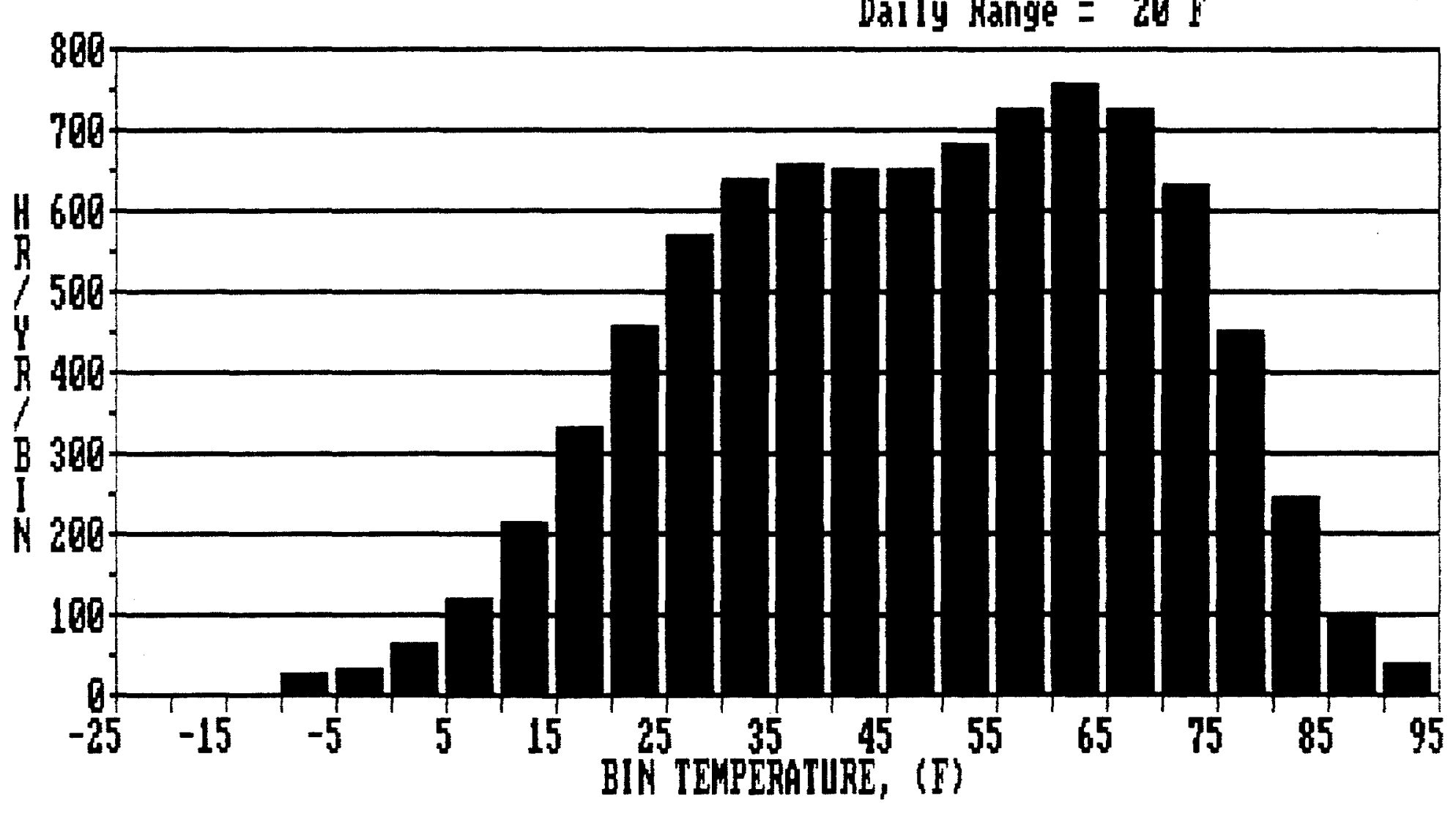


III.

FEFMILAE D-O EXFEFIMENTAL FACILITY SITE MAIN FING LAKE COOLING DATA

31. DCTOEEF, 1987

ANALYST: S.F. KFSTULOUICH

FEFMILAE CONSTFUCTION ENGINEEFING SEFVICES 
FEFUILAE STE GOOLIUG WTEF PFOFILES

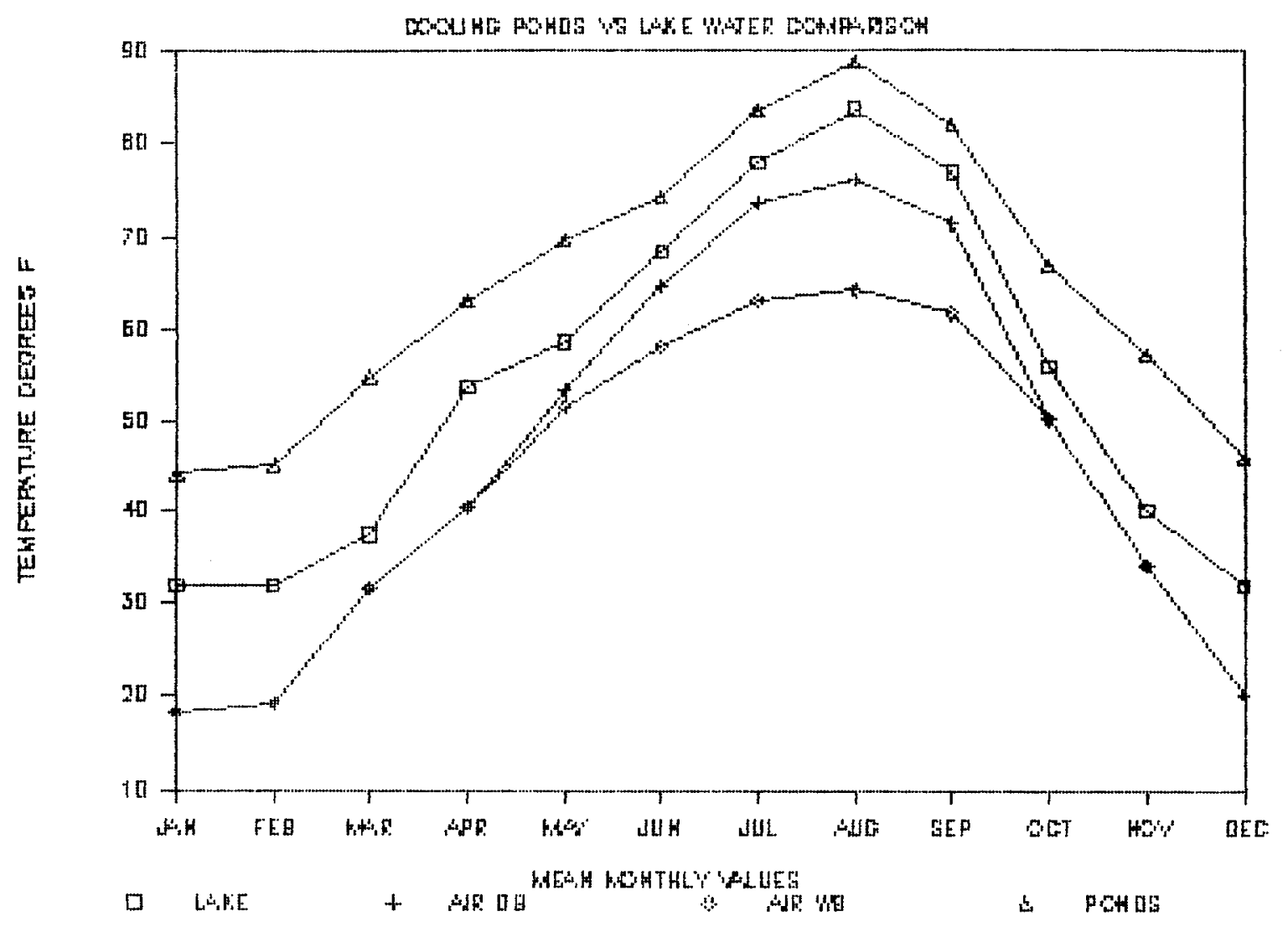

FERAILAE GITE COOLIMG WATER PROFILEE

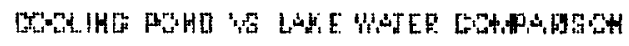

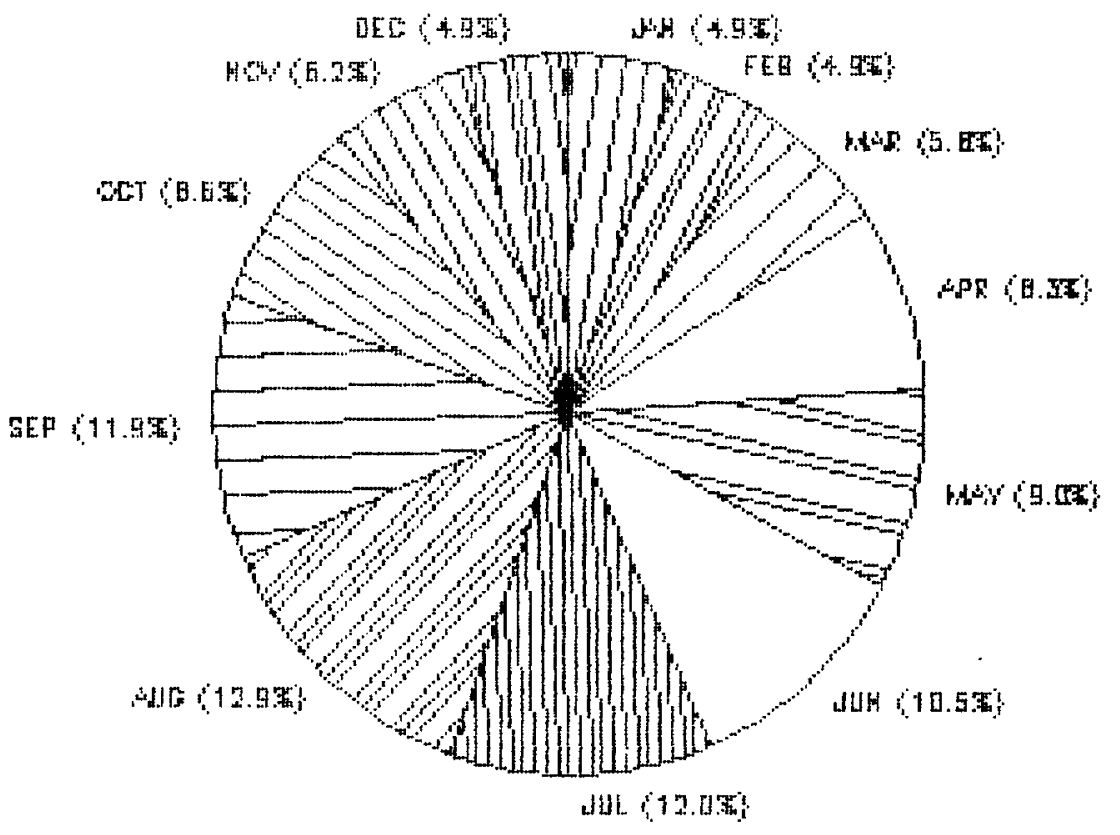




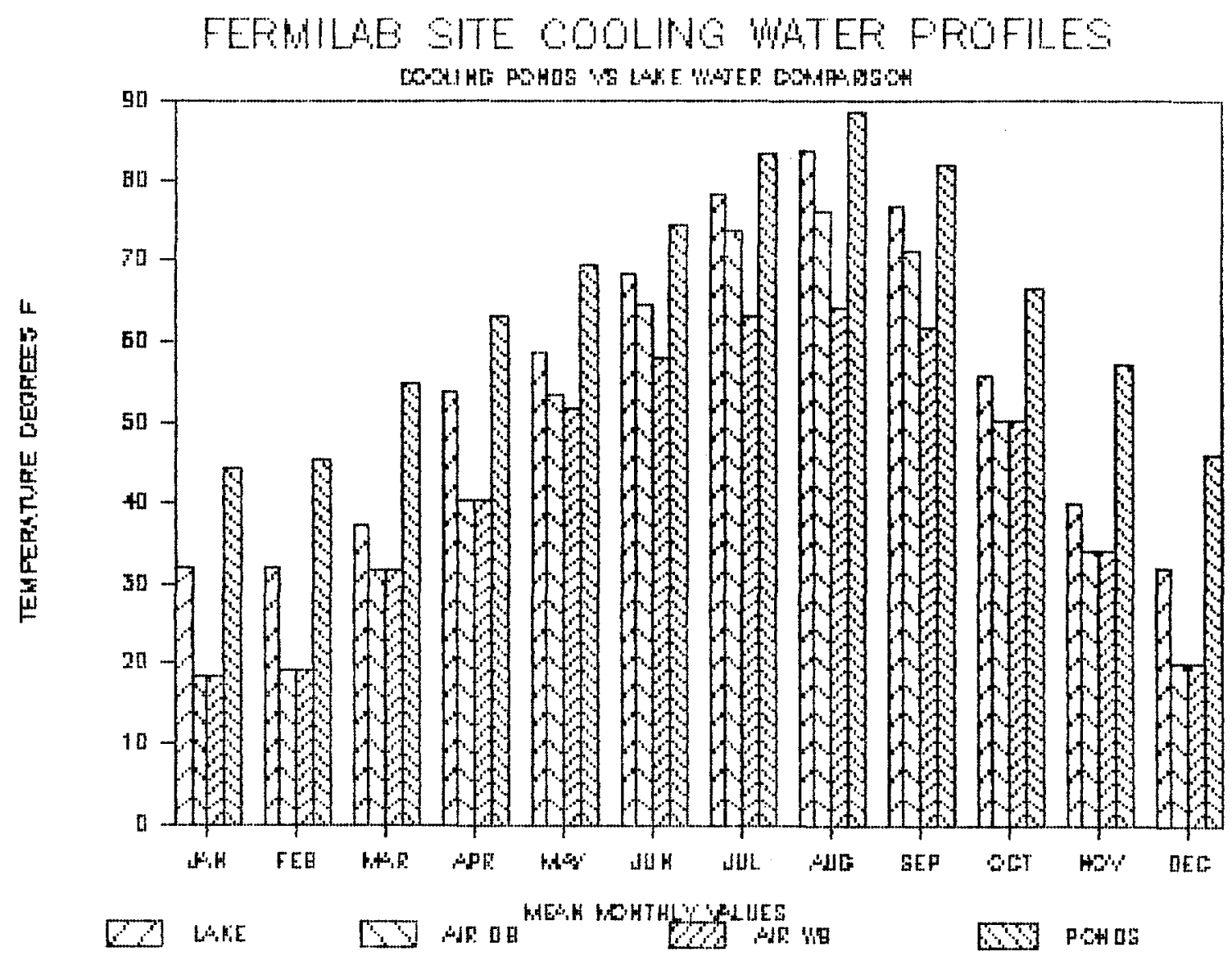


FEFMILAE ANWUAL STTE BURFACE WATEF FFOFILES

MEAN MONTH VALLES OF SIGNTFICANT INDICATOFS

LAKE WATEF TEMF

DA AIF TEMF DE

OA AIF TEMF WE

FOND WATEF TENF

\begin{tabular}{|c|c|c|c|c|c|}
\hline JAN & $\mathrm{FEE}$ & MAF: & $A F F^{i}$ & MAY & \\
\hline 22.0 & 200 & 37.4 & 53.8 & & 58.6 \\
\hline 18.3 & 19.2 & 31.6 & 40.4 & & 53.3 \\
\hline 18.3 & 19.2 & 31.6 & 40.4 & & 51.7 \\
\hline 44.5 & 45.2 & 54.8 & $6 \overline{S .1}$ & & 69.5 \\
\hline
\end{tabular}

\begin{tabular}{|c|c|c|c|c|}
\hline FLUE & $S E F$ & OCT & NOV & $\mathrm{DEC}$ \\
\hline $8 \% .7$ & 76.9 & 55.9 & $40 \times 1$ & 32.0 \\
\hline 76. & 71.3 & 50.3 & 34.2 & 20.1 \\
\hline 64. & 61.7 & $50 \cdot 2$ & 34.2 & 20.1 \\
\hline 88.9 & 82.1 & 66.7 & 57,2 & 46.1 \\
\hline
\end{tabular}


IV.

\author{
FEFIILAE DWO EXFEFUEMTAL FALILITY

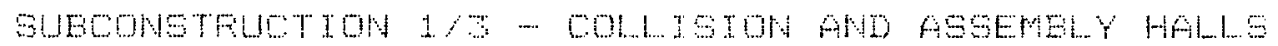

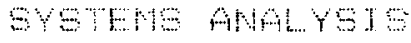

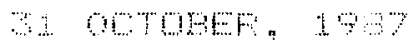 \\ कीम

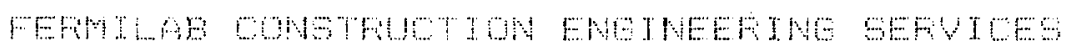


IV-a.

FEFMILAE D-O EXFEFIMENTAL FACILITY

SUECONSTFUCTION $1 / 3$ - COLLISION AND ASSEMELY HALLS EUILDING SECTOF: FHYSICAL. FAFAMETEFS AND DESCFIFTION

I OCTOEEF, 1987

ANALYST: S.F.FFSTULOUTCH

FEFMILAE CONSTFUCTION ENGINEEFING SEFUICES

IV -1 
Euilding Name : D-O SUECONSTFUET $1 / 3$

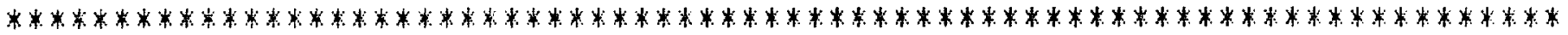

1. FIOUT CONDITIONS AUD FIOOF: AFEAS

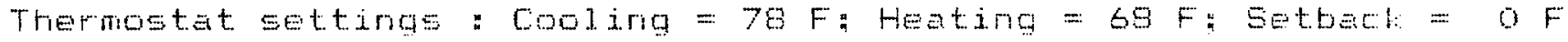
Wam-up factor $=0 \%$ Foom design melative rimidity $\%$ o $\%$

Floor Areas: Ferimeter = 24.400 Saft: Interior $=3$ oft Cooling provided during unoceupied period.

2. WALLS, FOOF, GLASS, SYYLIGHTS:

\begin{tabular}{|c|c|c|c|c|}
\hline Exposure & $\begin{array}{c}\text { Area } \\
(S q f t)\end{array}$ & $\begin{array}{c}\text { U-Factor } \\
\text { ETU: (hr-sqft-F) }\end{array}$ & $\begin{array}{c}\text { GIass Area } \\
(S q f t)\end{array}$ & $\begin{array}{l}\text { Glass U-Factor } \\
\text { BTU/(hr-sqft-F) }\end{array}$ \\
\hline North wall & $1, .700$ & 0.150 & 100 & 1.130 \\
\hline East Wall & $\because 100$ & 0.150 & 0 & $1 \cdot 1.50$ \\
\hline Southeast wal1 & 0 & 0.150 & 0 & 1.1 .50 \\
\hline South wall & 6,600 & 0.150 & 0 & 1.1 .0 \\
\hline West Wal] & 2.800 & 0.150 & 0 & 1.1 .20 \\
\hline Northwest WaII & 0 & 0.150 & 0 & 1. 1.0 \\
\hline Fer imeter Foof & 14.900 & 0,130 & \% & 0.550 \\
\hline Interior Fioof & 0 & 0.10 & 0 & 0.55 \\
\hline
\end{tabular}

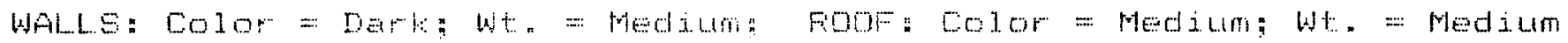

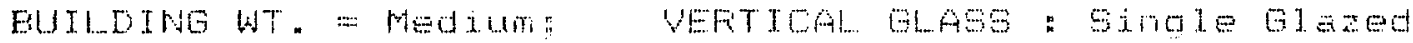

Shade Factors"

Internal shades are not used. y A weil ing olemum is not used.

\section{S. LIEHTS:}

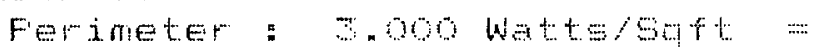

Interior : onoo Hattersatt =

Diversity Factors " Oocupied $=100 \%$ " umowempied $=100 \%$

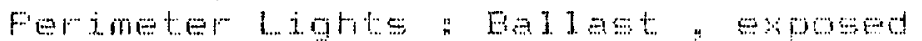

Triter"ior Lights " Eal Jast remesed

\section{3,200 watts Total \\ o wates Total}

4. MIOCELIANEOUS ELETTTOAL LOAOS:

\begin{tabular}{|c|c|c|c|c|c|c|}
\hline$F e r$ ineter & $\nabla c-c u p x \in 0$ & 2.455 & Watte/8at = & 60.00 & $\omega=t+=$ & Toted \\
\hline & unoceupied & 0.620 & Wat $t=150 t$ & 20,000 & watts $=$ & Totel \\
\hline Interior & oecupied & 1000 & Wattcs $/ 59+\mathrm{t}$ & 0 & wat & Total \\
\hline & Urocoupied & . & Wat $t=/ 50 \mathrm{ft}$ & 0 & wets & Totel 1 \\
\hline
\end{tabular}

5. FEOFLE LOADS

Total Decupancy $=2,440$ eqtaprsom $=\quad 10$ people total

Activity Level: 4. Medium wort:

Serisible = 295.0 ETU/hr/person: Latent $=455.0$ ETU/hm/person

Diversity Factors : Decupied $=70 \%$ Unoccupied $=20 \%$

6. MISCELLANEOUS INTEFNAL LODAOS:

$\begin{array}{llll}\text { Sensible : occupied }= & \text { o ETU/hr: Unoccupied }= & \text { BTu/hr } \\ \text { Latent : occupied }= & \text { OETU/hr: Unoccupied }= & \text { O BTU/hr }\end{array}$

7. WALLS ADJACENT TO NON-CONOTTIONED FEGTONS:

Wal1 Areas "Ferimeter = 21.5005 aft: Interior"w

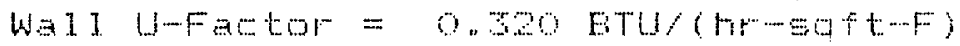

Adjacent region heating temperature $=60 \mathrm{~F}$

Adjacent region wooling temperature $=60 \mathrm{~F}$

๑. INFILTRATION DATA:

Ajr Flow Fates: 0woupied =-

200 efin: Urictopied $=$

$100, \mathrm{fm}$ 
IV-b.

\section{FEFMILAE D-O EXFEFTHENTAL FACILITY \\ SUECONSTFUCTION $1 / 3$ - COLLISION AND ASSEMELY HALLS BUILDTNG LOAD CALCULATIONS}

3 OCTOEEF, 1987

ANALYST: S.F.FFSTULOVICH

FEFMILAE CONOTFUCTION ENGINEEFING SEFVICES 
LOAD FGOFILE SUMMAFY

Bujlding Name : D-O SUECORGTFUCT $1 / 3$ (Complex)

Site Name : AUFOFA, IL FEFMT, User Defined

Dete: $: 1.1-16-87$

601178620

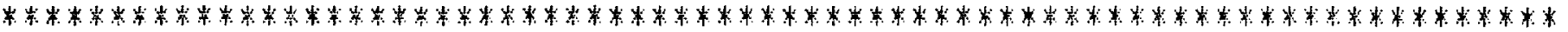

TABLE 1. OCLUFIED FEFIOD (LOEd doE not jrolude vent. load, fan reat)

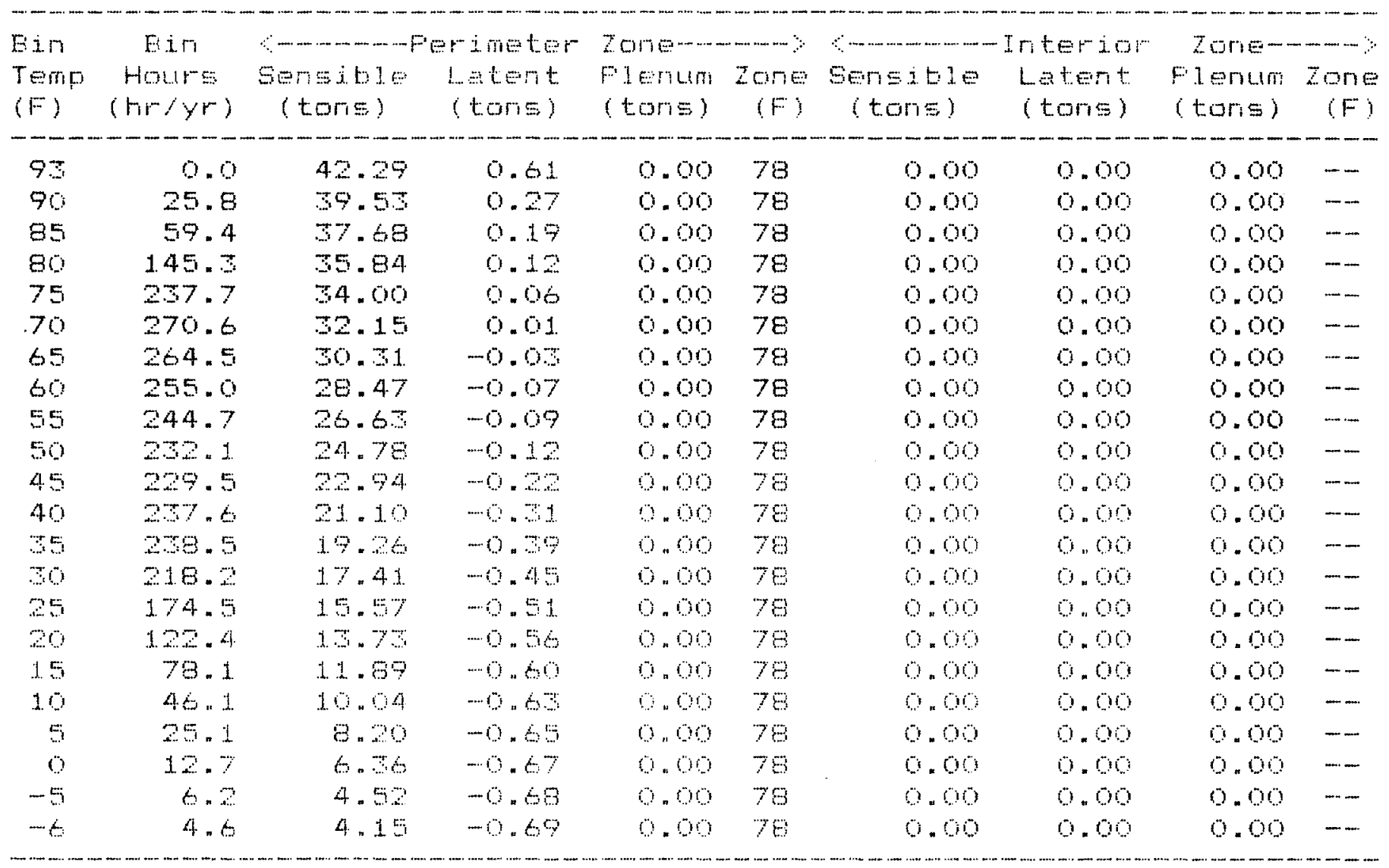

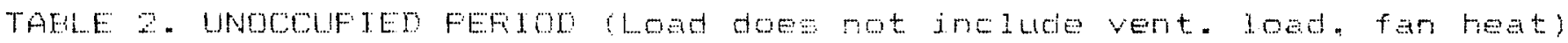

\begin{tabular}{|c|c|c|c|c|c|c|c|c|c|}
\hline 9 & 14.6 & 27.92 & 0.08 & 9 (y) & $7 \theta$ & 0.90 & i & 0.00 & -- \\
\hline 85 & 26.5 & 26,12 & 0,04 & 0.00 & 78 & 0.60 & 0.00 & 0.00 & $\ldots-$ \\
\hline 80 & 99. & 24.3 & 0,00 & 0,0 & 78 & 0.00 & 0,00 & 0.0 & -- \\
\hline 70 & 95.6 & 20,76 & -0.06 & 0.00 & 78 & 0.00 & 0. ब0 & o. & -- \\
\hline 65 & $46 \%, 7$ & 18.9 & -6.07 & 0.0 & 78 & 9.0 & 0. & 0.90 & -- \\
\hline 60 & 500.4 & 17.14 & -0.09 & 0.00 & $7 \mathrm{e}$ & 0.00 & 0.00 & 0.00 & $-\cdots$ \\
\hline 50 & 447.6 & 13.54 & -0.12 & 0.00 & 78 & 0.00 & 0.00 & 0.00 & - \\
\hline 45 & 422.7 & 11.74 & -0.17 & 0.00 & 78 & 0.00 & 0.00 & 0.00 & -- \\
\hline 40 & 41.3 .7 & 9.95 & -0.21 & 0.00 & 78 & 0.00 & 0.00 & 0.00 & -- \\
\hline 55 & 417.0 & 8.15 & -0.25 & 0.00 & 78 & 0.00 & 0.00 & 0.00 & -- \\
\hline 30 & 417.4 & 6.5 & -0.28 & 0.00 & 78 & 0.00 & 0.00 & 0.00 & -- \\
\hline 25 & 395.2 & 4.56 & -0.31 & 0.00 & 78 & 0.00 & 0.00 & 0.00 & -- \\
\hline 5 & 96.3 & 0.00 & 0.00 & 0.00 & $\ldots$ & 0.00 & 0.00 & 0.00 & -- \\
\hline 0 & 50.9 & 0.90 & 0.00 & 0.03 & $-n \cdot \cdots$ & i & 0,00 & 0.00 & -- \\
\hline-5 & 25,7 & 0.00 & 0.00 & 0.0 & $\cdots \cdots$ & 0.00 & 0.90 & 1. & -- \\
\hline-6 & $21 \cdot 1$ & 0.0 & 0.00 & 0.00 & $\cdots \cdots$ & 0.00 & 9.80 & 0,00 & $-\cdots$ \\
\hline-6 & 0.0 & -30.67 & $4 \times$ & 9.9 & $6 \theta$ & व. ओ & 1) & 0.90 & $-\cdots$ \\
\hline
\end{tabular}


Building Name : L-O SUECONSTFUCT 1/J (Complex)

TABLE 1. SOLAR GAINS EY EXFOSUFE

\begin{tabular}{|c|c|c|c|c|c|c|c|c|c|c|}
\hline \multicolumn{3}{|c|}{$\begin{array}{l}\text { Bin } \\
\text { Temp }\end{array}$} & \multicolumn{6}{|c|}{$\begin{array}{c}\text { Solar Gains by Exposure } \\
\text { (ETU/(hr-sqft)) }\end{array}$} & \multirow{2}{*}{$\begin{array}{l}\text { Ferim } \\
\text { Horiz }\end{array}$} & \multirow{2}{*}{$\begin{array}{l}\text { Inter } \\
\text { Horiz }\end{array}$} \\
\hline$(F)$ & North & $N E$ & East & $S E$ & South & $S W$ & West & NW & & \\
\hline 9.5 & 15.0 & 30.4 & 47.4 & 5.6 & 50.4 & 74.4 & 56.9 & 27.5 & 0.0 & 0.0 \\
\hline 90 & 9.2 & 21.5 & 5.6 & 38.1 & 55.9 & 52.9 & 40.4 & 19.5 & 0.0 & 0.0 \\
\hline 85 & 8.8 & 20.4 & 2.0 & 36.3 & 34.4 & 50.5 & 38.6 & 18.5 & 0.0 & 0.0 \\
\hline 80 & 8.3 & 19.5 & 30.3 & 34.6 & 32.9 & 48.1 & 36.7 & 17.5 & 0.0 & 0.0 \\
\hline 75 & 7.8 & 18.2 & 28.7 & 32.9 & 31.4 & 45.8 & 34.9 & 16.5 & 0.0 & 0.0 \\
\hline 70 & 7.4 & 17.1 & 27.1 & 51.2 & 29.9 & 45.4 & 3.0 & 15.5 & 0.0 & 0.0 \\
\hline 65 & 6.9 & 16.0 & 25.4 & 29.4 & 28.4 & 41.0 & 31.2 & 14.5 & 0.0 & 0.0 \\
\hline 60 & 6.5 & 14.9 & 23.8 & 27.7 & 27.0 & 58.6 & 29.5 & 15.5 & 0.0 & 0.0 \\
\hline 55 & 6.0 & 1.8 .8 & 22.2 & 26.0 & 25.5 & $\therefore 6.5$ & 27.5 & 12.6 & 0.0 & 0.0 \\
\hline 50 & 5.5 & 12.6 & 20.5 & 24.2 & 24.0 & 3.9 & 25.6 & 11.6 & 0.0 & 0.0 \\
\hline 45 & 5.1 & 11.5 & 18.9 & 22.5 & 22.5 & 31.5 & 25.8 & 10.6 & 0.0 & 0.0 \\
\hline 40 & 4.6 & 10.4 & 17.9 & 20.8 & 21.0 & 29.1 & 21.9 & 9.6 & 0.0 & 0.0 \\
\hline 5 & 4.1 & 9.5 & 15.6 & 19.0 & 17.5 & 26.8 & $20 \cdot 1$ & 8.6 & 0.0 & 0.0 \\
\hline 30 & $\therefore .7$ & a.2 & 14.0 & 17.5 & 10.0 & 24.4 & 18.2 & 7.6 & 0.0 & 0.0 \\
\hline 25 & 32 & 7.1 & 12,4 & 15.6 & 16.5 & 22.0 & 16.4 & 6.6 & 0.0 & 0.0 \\
\hline 20 & 2.8 & 6.0 & 10.7 & 13.9 & 15.0 & 19.6 & 14.5 & 5.6 & 0.0 & 0.0 \\
\hline 15 & 2.3 & 4.9 & 9.1 & 12.1 & 13.5 & 17.3 & $12 \times 7$ & 4.7 & 0.0 & 0.0 \\
\hline 10 & 1.8 & $\therefore .8$ & 7.5 & 10.4 & 12.0 & 14.9 & 10.8 & 3.7 & 0.0 & 0.0 \\
\hline 5 & 1.4 & 2.7 & 5.8 & Q.7 & 10.6 & 12.5 & 9.0 & 2.7 & 0.0 & 0.0 \\
\hline 0 & 0.9 & 1.6 & 4.2 & 6.9 & 9.1 & 10.1 & 7.1 & 1.7 & 0.0 & 0.0 \\
\hline-5 & 0.4 & 0.5 & 2.6 & 5.2 & 7.6 & 7.8 & 5.3 & 0.7 & 0.0 & 0.0 \\
\hline-6 & 0.8 & 0.2 & 2.3 & 4.9 & 7.3 & 7.3 & 4.9 & 0.5 & 0.0 & 0.0 \\
\hline-6 & 0.0 & 0.0 & 0.0 & 0,0 & 0.0 & 0.0 & 0.0 & 0.0 & 0.0 & 0.0 \\
\hline
\end{tabular}


ETD SUMMAFY

Euildirig Nane : D-O SUECONSTFUCT 1/S (COMPlex)

Date: $11-16-87$

Site Name: AUFOFA, IL FEFMI, USER Defined 60117862.0

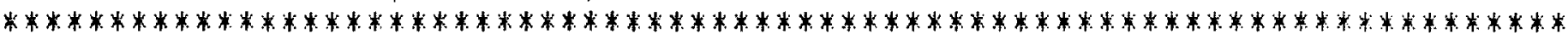
TAELE 1. DCLUFIED FEFIOD

Eiri

Temp

(F

Equivalent Temperature Differences

Ey Exposure

\begin{tabular}{|c|c|c|c|c|c|c|c|c|c|c|}
\hline 93 & 8.0 & 11.4 & $1 \% .8$ & 25.5 & 29.8 & 27.0 & 20.7 & $10 . \overline{5}$ & 50.4 & ---- \\
\hline 90 & 5.4 & 7.8 & 9.5 & 16.4 & 20.8 & 18.9 & 14.4 & 7.0 & 21.7 & ---- \\
\hline 85 & 1.0 & $\Xi$ & 4.9 & 11.5 & 15.7 & 13.9 & 9.6 & 2.5 & 16.4 & $--\cdots$ \\
\hline 80 & -3.4 & -1.5 & 0.3 & 6.6 & 10.5 & 8.9 & 4.7 & -2.0 & 11.1 & $--\cdots$ \\
\hline 75 & -7.8 & -5.8 & -4.3 & 1.7 & 5.4 & 3.9 & -0.1 & -6.5 & 5.8 & $-\cdots-\cdots$ \\
\hline 70 & -12.2 & $-10,3$ & -8.9 & -7.2 & 0.2 & -1.1 & -4.9 & -10.9 & 0.5 & ---- \\
\hline 65 & -16.6 & -14.8 & -1.5 & -8.1 & -4.9 & -6.1 & -9.8 & -15.4 & -4.9 & ---- \\
\hline 60 & -21.0 & -19.7 & $-1 \theta .1$ & $-13 \cdot 0$ & -10.1 & -11.1 & -14.6 & -19.9 & -10.2 & $---\cdots$ \\
\hline 55 & -25.4 & $-2 \square .8$ & -22.7 & -17.9 & -15.2 & $-16 \cdot 1$ & -19.4 & -24.4 & -15.5 & $-\cdots-\cdots$ \\
\hline 50 & -29.8 & -20.4 & -27.3 & -22.8 & -20.4 & $-21=1$ & -24.2 & -20.8 & -20.8 & \\
\hline 45 & $-\because 4.2$ & -22.9 & -31.7 & .27 .7 & -25.5 & $-26 \cdot 1$ & $-29,1$ & -3.5 & -26.1 & ---- \\
\hline 40 & -38.6 & -37.4 & $-36,5$ & -72.5 &. .90 .7 & -1.1 & -3.5 & -37.8 & -31.4 & ---- \\
\hline$\because 5$ & -4.0 & -41.9 & -41.1 & 97,4 & -35.8 & -36.1 & -50.7 & $-42 \cdot 3$ & -56.7 & \\
\hline$\because 0$ & -47.4 & -46.4 & -45.7 & -42 & -41.0 & -41.1 & $-4 \pi \cdot 6$ & -46.7 & -42.0 & \\
\hline 25 & $--m-\cdots$ & $-\cdots-\cdots$ & $-\cdots-\cdots$ & - & $-m$ & $--\cdots$ & 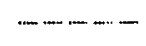 & $-\cdots-\cdots$ & $-\cdots---$ & ----- \\
\hline 20 & $-\cdots$ & $---\cdots$ & 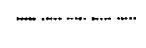 & $\ldots$ & 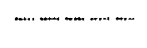 & $-\ldots$ & $-\cdots-\cdots$ & $---\cdots$ & 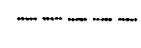 & -- \\
\hline 15 & $-\cdots-\cdots$ & $-\cdots-\cdots$ & $--\cdots$ & 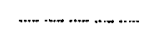 & 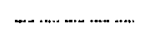 & 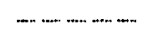 & $-\cdots$ & $\cdots$ & $-\ldots-\ldots$ & $-\cdots$ \\
\hline 10 & -54.9 & -54.5 & -54.1 & -5.5 .9 & -51.6 & $-51,1$ & -52.9 & -54.6 & -5.2 .2 & ---- \\
\hline 5 & -59.3 & -.59 .0 & -58.7 & -56.8 & -56.7 & $-56 \cdot 1$ & -57.7 & -59.1 & -58.6 & ---- \\
\hline 0 & -6.7 & -6.5 & $-6 \square=3$ & -61.7 & -61.9 & $-61,1$ & -62.5 & -6.0 & -6.9 & $-\cdots-\cdots$ \\
\hline-5 & $-68 \times 1$ & -68.1 & -67.9 & $-66 \cdot 6$ & -67.0 & $-66 \cdot 1$ & -67. & $-68 \cdot 1$ & -69.2 & $-\cdots-\cdots$ \\
\hline-6 & -69.0 & -69.0 & -60.8 & -67.5 & -60.0 & -67.1 & -68. & $-6 \% .0$ & -70.2 & ----- \\
\hline
\end{tabular}

TAELE 2. UNOCOUFIED FEFIOD

\begin{tabular}{|c|c|c|c|c|c|c|c|c|c|c|}
\hline 90 & 5.4 & 7.8 & 9.5 & $16 n 4$ & 20.8 & 18.9 & 14.4 & 7.0 & 21.7 & ---- \\
\hline 85 & 1.0 & $y$ & 4.9 & 1.1 .5 & 15.7 & $13 \%$ & 9.6 & 2.5 & 16.4 & ----- \\
\hline $8 \%$ & -3.4 & $-1, \pi$ & 0.5 & 6.5 & 10.5 & 8.9 & 4.7 & -2.0 & 11.1 & - \\
\hline 75 & -7.8 & $-5,8$ & $-4, \because$ & 1.7 & 5.4 & $7 . y$ & -0.1 & -6.5 & 5.8 & 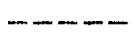 \\
\hline 70 & -12.2 & -10.3 & -8.9 & $-\square, 2$ & 0.2 & $-1,1$ & -.4 .7 & -10.9 & 0.5 & ----- \\
\hline 65 & -16.6 & -14.8 & -13.5 & $-8,1$ & -4.9 & -6.1 & -9.8 & -15.4 & -4.9 & \\
\hline 60 & -21.0 & $-19 . .7$ & -18.1 & $-1 \div 0$ & $-10 \cdot 1$ & -11.1 & -14.6 & -19.9 & -10.2 & \\
\hline 55 & -25.4 & $-2 \div .8$ & -22.7 & -17.9 & -1.5 .2 & -16.1 & -1.9 .4 & -24.4 & -15.5 & $---\cdots$ \\
\hline 50 & -29.8 & -28.4 & -27.5 & -22.8 & -20.4 & -21.1 & -24.2 & -28.8 & -20.8 & $--\cdots$ \\
\hline 45 & ---- & ----- & $----m$ & ------ & $---\cdots$ & $---\cdots$ & ----- & ---- & ----- & ----- \\
\hline 40 & ---- & $---\cdots$ & $----\cdots$ & ----- & $-\cdots-\cdots$ & $--\cdots$ & 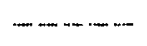 & $--\cdots$ & ----- & \\
\hline 55 & $-\cdots-\cdots$ & 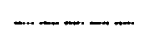 & $-\cdots+\cdots$ & $-\cdots-\cdots$ & $---\cdots$ & $---\cdots$ & $--\cdots$ & $\cdots---$ & ----- & $---\cdots$ \\
\hline 50 & $--\cdots$ & $-\cdots+-\cdots$ & $--\cdots$ & $-\cdots-\cdots$ & - & $-m--\cdots$ & $-\ldots-m-n$ & $-\cdots \cdots$ & $--m-m-m$ & $--\cdots$ \\
\hline 25 & -41.8 & -41.0 & -40.5 & -37.2 & $-\exists 6.1$ & $-36 \cdot 1$ & -38.4 & -41.2 & -37.3 & $-\cdots$ \\
\hline 20 & -46.2 & -45.5 & -44.9 & -42.1 & $-41 . \Xi$ & $-41 \cdot 1$ & $-4 \div, 2$ & -45.7 & -42.6 & $-\cdots-\cdots$ \\
\hline 15 & -50.5 & -50.0 & -49.5 & -47.0 & -46.4 & -40.1 & $-4 B .0$ & -50.2 & -47.9 & $--\cdots$ \\
\hline 10 & -54.9 & -54.5 & -54.1 & -51.9 & -51.6 & $-51 \cdot 1$ & -52.9 & -54.6 & -5.5 & ----- \\
\hline 5 & -59. & -59.0 & -50.7 & $-56,8$ & $-56,7$ & $-5, \ldots 1$ & -57.7 & $-5 \%=1$ & -58.6 & 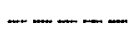 \\
\hline 0 & $-6 \square .7$ & -63.5 & -63.5 & -61.7 & -61.9 & $-61=1$ & $-62 \cdot 5$ & -63.6 & -6.9 & $-\ldots$ \\
\hline-5 & -68.1 & -68.1 & -67.9 & -66.6 & -67.0 & $-6 \dot{0}, 1$ & -67.3 & $-6 \theta .1$ & -69.2 & ----- \\
\hline-6 & -69.0 & -69.0 & $-6 \theta .8$ & $-67 \times 5$ & -68. & $-67,1$ & $-60, \because$ & -69.0 & -70.2 & ----- \\
\hline-6 & -74.0 & -74.0 & -74.0 & -74.0 & -74.0 & -74.0 & -74.0 & -74.0 & -74.0 & $\cdots$ \\
\hline
\end{tabular}


IV-C.

FEFMILAE DWO EXFEFTMENTAL FACILITY

SUECONSTFUETION $1 / 3$ - COLLISTON AND ASSEMELY HALLS SYSTEM COMHAISON FEFFOFIANCE SIMLLATIONS

I. OOTOEEF, 1907

ANALYST" ${ }_{\text {"F }}$.AFETULOVICH

FEFMTLAE CONOTRUCT ION ENGTHEEFING SEFUTCES 
IV-C. -1 .

FEFHIAE DWO EXFEFTMENTAL. FACILITY

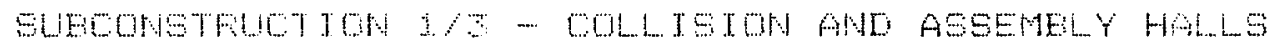

OFEFAT IOHAL FEFOFMANE SIMULATIOH

DESIGU BASIS SYSTEM

PETTFEOATING WHLLES ANO OENTRAL STATTON AHU

WTH THESE ENEFOY FEATLFES:

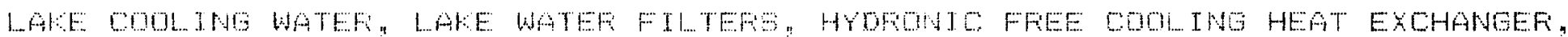

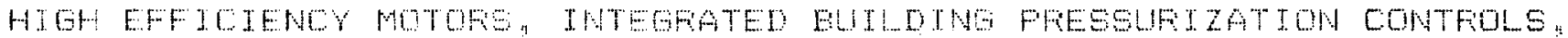

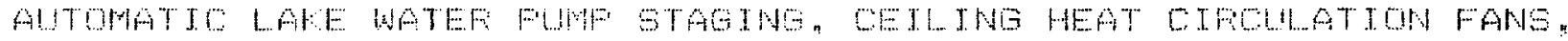

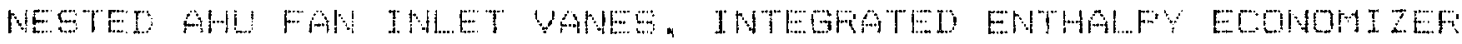

-1 OCTOFE, $199 \%$

ANALYI:

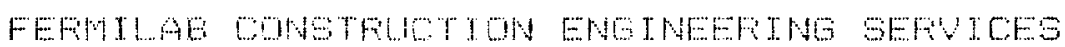




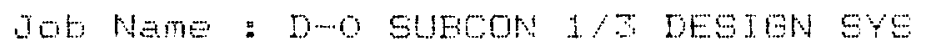

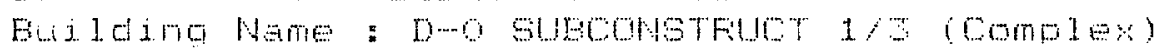
Fage 1

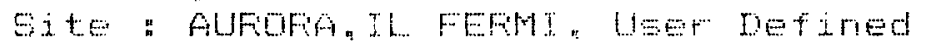

sedpe of maj.

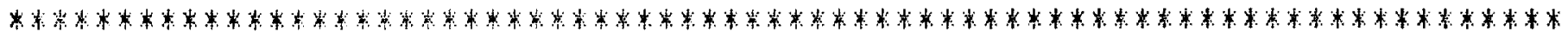

A. ATF HANDL TMO SYGEM

1. FEFIMETEF TEFMINAL TYFE : CONSTANT YOLUPE

Supply far type : Eastuard inelined or airfoid with inlet vares

Suppiy fan totaj. statio prestre $=$ mod ir. wo.

Fieturn fan type = Controlled pitah axial.

Fieturn fan total static pressure = 1.75 in. 19 .

Design supply aif $=42000$ efm gb7 F. Vent. ait= $9000 \mathrm{~cm}$

fre cooling terminals used for heating $?$ CY

Economizer type : Integrated, enthelpy controlled

IS a ventilation reclaim device used ? CN

Are ventilation ar dampers closed for unoceunied periods ? lN

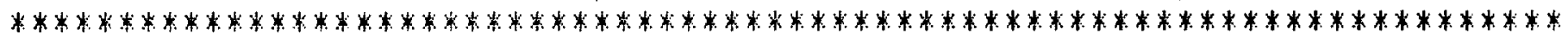

B. HEATING F!_ANTS

\% 1 . FEFIMETEF FLANT : ELECTFICAL FESISTANCE

IE rydromie hegtim used

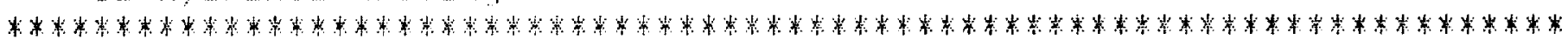




\section{SYSTEM INFUT SUMMAFY}

Job Nane : D-O SURCON $1 / 3$ DESIGN SYS

Building Name : D-0 SUECONSTFUCT $1 / \mathrm{s}$ (Complex)

Fage 2

Date: $11-16-87$

60117862.0

Site : AUFOFA, IL FEFMI, USEr Defined

Scope of Analysis: Cooling and Heating Systems

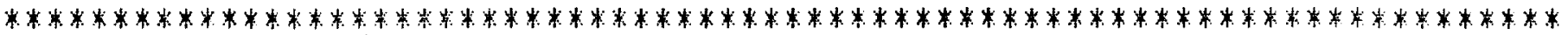

C. COOLING FLANTS

1. FEFIMETEF FLANT : FECIFFOCATING WATEF COOLED

Capacity a $85 \mathrm{~F}$ entering water $=75.0$ tons

$\mathrm{kW} /$ Ton G $85 \mathrm{~F}$ entering water $=0.85 \mathrm{kw} / \mathrm{ton}$

Is hydronic cooling used ? CY

Is chilled water reset used $?$ dy

Is not gas bypass used ? N

Is condenser performance altitude adjusted ? $Y$ ?

Is there one compressor per condenser circuit ? $? \mathrm{y}$

Are compressoms cycled $? \mathrm{r}$

Heat sink type : Open cooling tower

Minimum entering water temperature $=3 \mathrm{~F}$

Is a hydronic economizer used $?$ Y $Y$; Economizer efficiency $=80 \%$

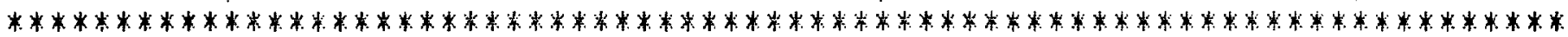

D. FUMFING SYSTEMS

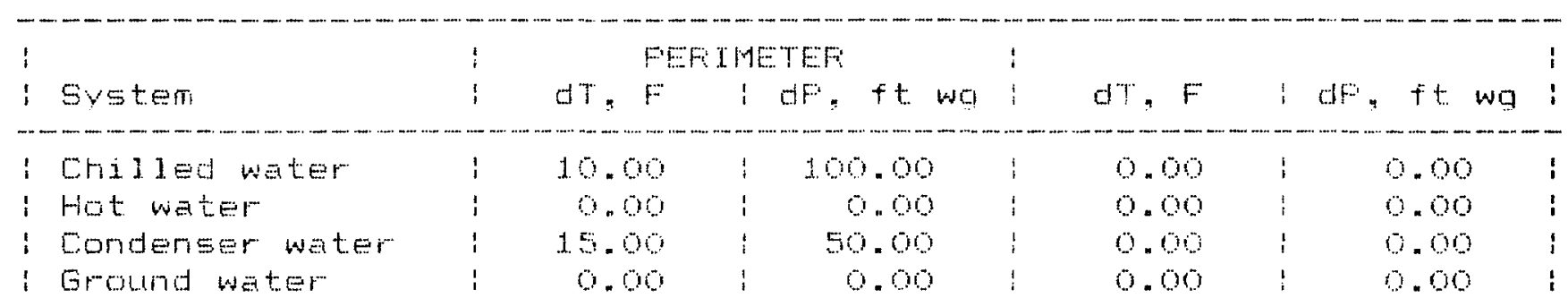

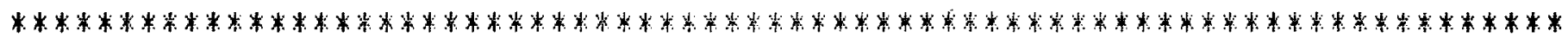

E. NON W HAC SYSTEMS

1. NON-HVAC ELEETFICAL ENEFEY UEE
Lighting tw: Ocoupied =
$7 x_{2}$ tw: Unocoupied =
$73210 \%$
Misce 1 : : Occupied =
60.0 ka: Unoceupied =
$20.010 \mathrm{w}$
Other l:w : Dectoied =
0.0 la: Uroecupied =:
a. $10 \omega$

2. DHW SYSTEM (Not utilized im trie system)

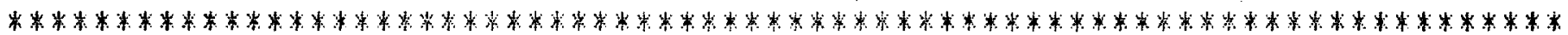

F. FUEL COSTS

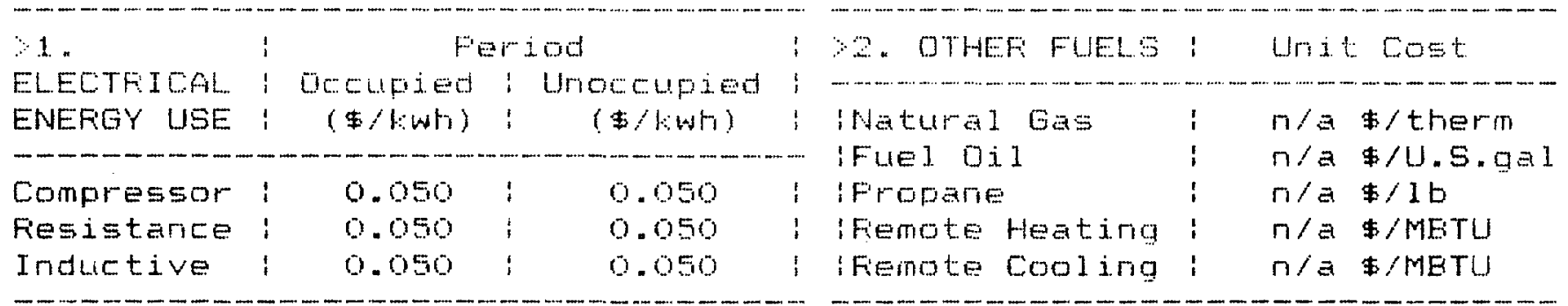


TOTAL EUTLDING ENEFGY SUMMAFY

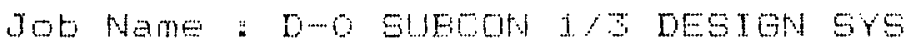

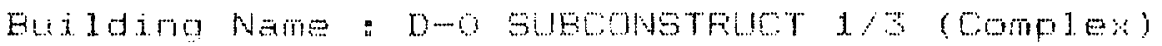

Feriod:Al]

Site "AUFOFA, IL FEFIV "Wser Defined

Zone : Elock

Dati: ; $11-16-97$

Serial Number:

60117662.0

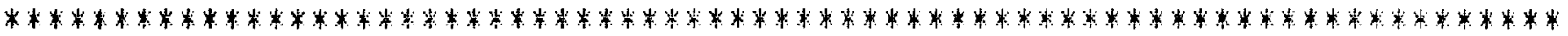

HVAC EMEFGY

Comporimt

Anलut 1

Cost

$\left.(s ; y)^{\prime}\right)$

Electric (Oco)

Electric (Unoce)

Natural Ges

Fue 1 Oil

Fropane

Fiemote Heating

Fiemote Coolimg

HVAC Total.
Energy or Fue?

Units:

Coristined

7855

1.3612

0

6

\section{0}

)

0

21467

272247 fwh y

o $1 \mathrm{~b} / \mathrm{yr}$
DOE FIUF DOE FITF (1000 BTU) (1000 BTU)

- - - - - - - - - - - - -

\section{1 lwh/yr}

o therms/yr

o U.S. ga1/vr

O METU YY

O MBTU/Yr"

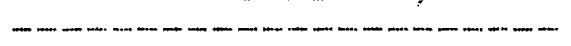

$\begin{array}{rr}0 & 0 \\ 4980516 & 1465.29\end{array}$

1822255

5.56152

929176

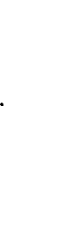

NOH-HVAC: ENEFGY

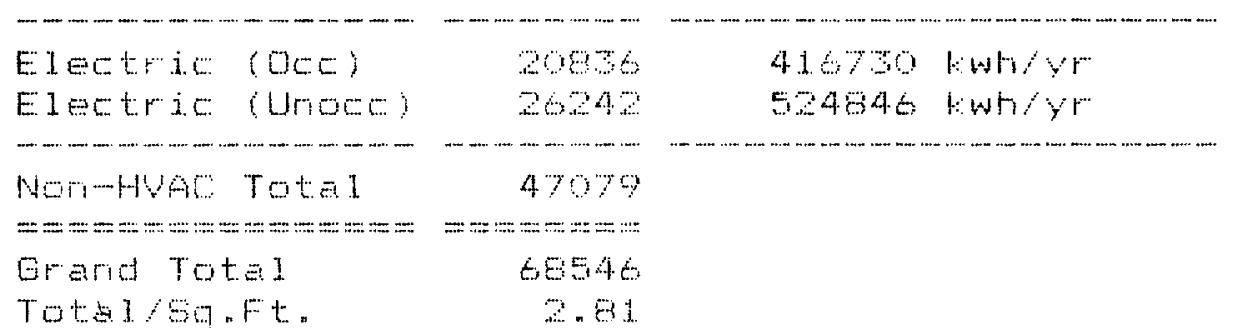

Tots] $180 . F t . \quad 2.81$

$\begin{array}{rr}4834069 & 1422298 \\ 6088219 & 1791201 \\ 10922282 & -21299 \\ -15902597 & 4678928 \\ -651.75 & 191.76\end{array}$

HWA Bummery

\section{HAE: Totel [ost}

$=\quad 0.8 \theta+/ 8 q . F+\ldots / \%$

Total HYaC Elemtacel Eneroy 1. 2\% " of Total Cost

The

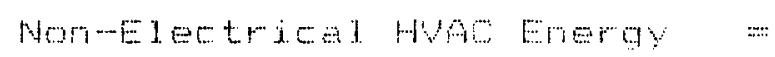

1.7.60 kwh/89.Ft. . Y Y

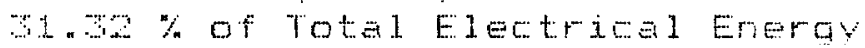
$-0.00 \%$ of Total Erieroy

tey

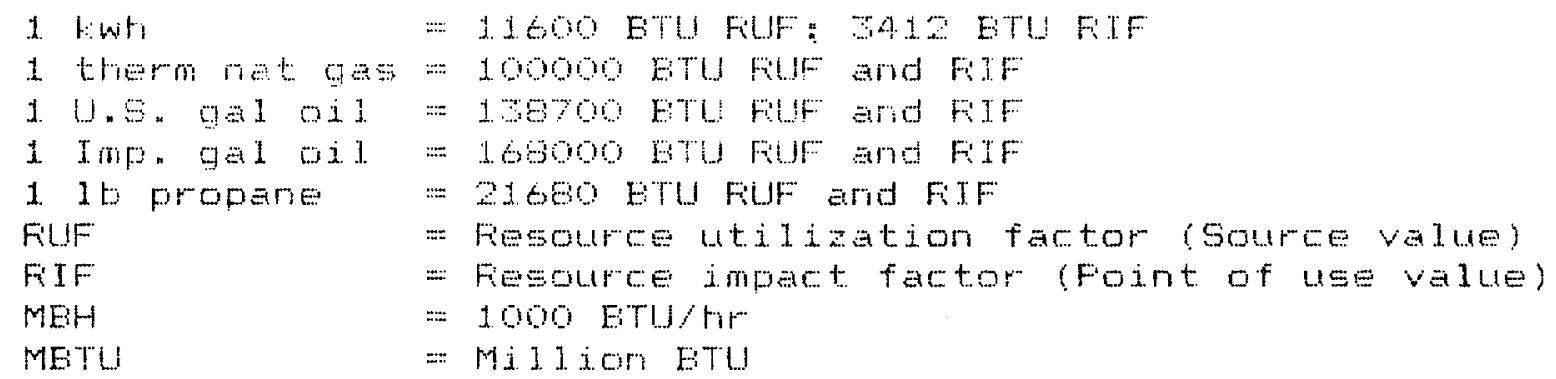


Jot Name : D-O SUECON $1 / 3$ DESIGN SYS

Euilding Name : D-O SUECONSTFUCT $1 / 3$ (Complex)

Site : AUFOFA, IL FEFMI, UeEr Detined

$$
\text { Date : } \begin{array}{r}
\text { Fog } 1 \\
11-16-87 \\
60117862.0
\end{array}
$$

Scope of Amelysis " Cooling ard Heating Systems

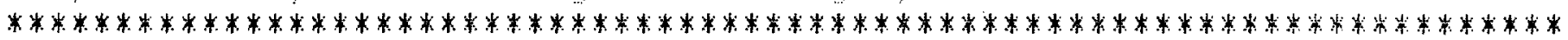

A. AIF HANDLING EYETEMS (ANNUALL FAN OFEFATING COST =

$\$ 20,043$

1. FEFIMETEF TEFMINAL TYFE: CONSTANT VOLUME

Supply fan type : Eactward inclined or airfoil with inlet vanes

Supply fan total static pressure $=3.00 \mathrm{in}$. wg.

Fieturn fan type : Controlled piteh axial

Feturn fan total static pressure $=1.75$ in. $w g$.

Design supply air $=42000$ efm e $67 \mathrm{~F}$ : Vent. air= $3000 \mathrm{cfm}$

Are cooling terminals used for heating ? CY

Economizer type : Integrated, enthalpy controlled

Is a ventilation reclaim device used?

Are ventilation air dampers closed for unoccupied periods $?$ N?

*********************************************************************************

E. HEATING FLANTS (ANNUAL HEATING FLANT DFEFATING CDST =

$\$ 498)$

$>1$. FEFIMETEF FLANT : ELECTAICAL RESISTANCE

Is hydronic heating used ? N

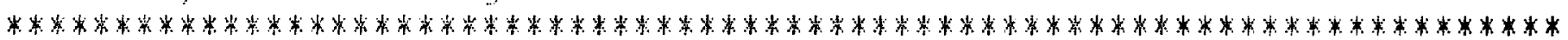




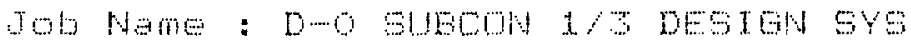

Euilding Name : D-o suedonetfuct $1: 3$ (Complen)

Fage 2

Site : AUFOFA, RL. FEFMI, User Defined

Date: $: 11-16-67$

60117862.0

Scope of Anelysi: Coolding and Heating Syeteme

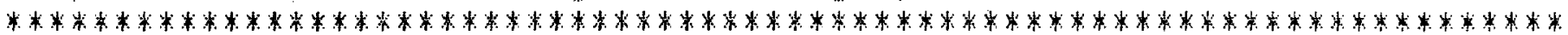

C. COOLING FILANTE IANVIAL COOLING PLANT OFERATING COET =

\$580

1. FERIMETEF FLANT : REOPFOUATING WATEF CODLED

Capacity a 85 F entering water $=75.0$ tons

KW/Ton e $85 \mathrm{~F}$ entering water $=0.85 \mathrm{kw} / \mathrm{ton}$

Is hydronic cooling beed $?$ Yy

Is chilled weter reset used ? r w

Is hot qas bypass lised ? U

Is condenser performance eltitude adjusted $?<Y$

Is there one compressor per condenser circuit $?<y$

Are compressors cyeled ? ry

Heat sink type: Open cooling tower

Minimum entering water temperature $=32 \mathrm{~F}$

Is a hydronic economizer used 7 y : Economizer efficiency $=80 \%$

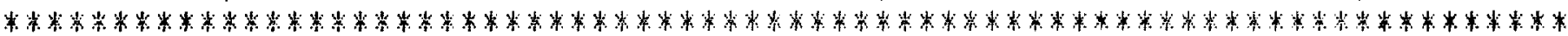

D. FUMATHE SYSTEHS

(ANANUAL PUMF DFEATING OOST =:

$+46)$

\begin{tabular}{|c|c|c|c|c|c|c|c|c|}
\hline & i & & 19 & $7 m$ & & & & \\
\hline systm & $i$ & His & i & $d F=f t \omega G$ & $i$ & $d T, F$ & $d F=$ & WO \\
\hline Cridlad water & $i$ & 10.6 & ! & $100 \cdot 90$ & & 9.9 & 1 & $00^{\circ}$ \\
\hline Hot water & $!$ & T. & 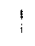 & o. & i & ओं & $i$ & $\mathrm{OCO}^{\mathrm{O}}$ \\
\hline 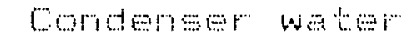 & $i$ & 15.60 & $i$ & 50,0 & 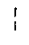 & 0.0 & $i$ & 0.00 \\
\hline Grobime water & i & 9.96 & $\vdots$ & 0.0 & ; & 0.00 & $:$ & 0.00 \\
\hline
\end{tabular}

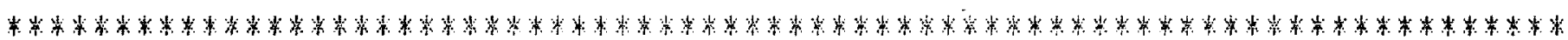

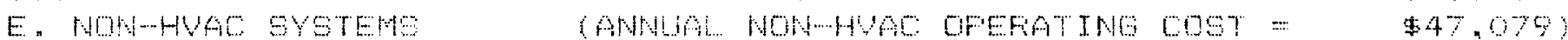

1. NON HUAC ELECTETCGL ENEPCY UEF

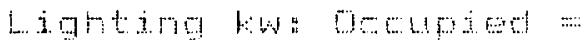
7 . 2 tw: Unoweupled $=$
$7+2 \mathrm{kN}$

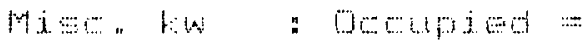
60.0 kw: Unow upied =
other ta a oreupied =
0.0 tw: Unocenpled =
$20.010 \mathrm{w}$
o. $1: 4$

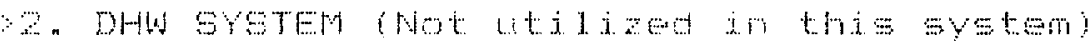

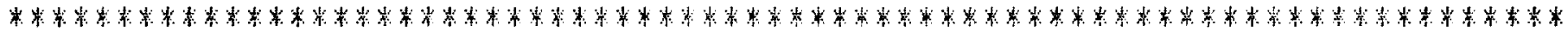
F. FUEl TOPS

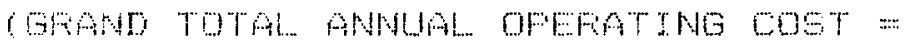

$+60.546)$

\begin{tabular}{|c|c|c|c|c|c|}
\hline$x_{n}$ & $i$ & \multicolumn{3}{|c|}{ Fepiot } & \\
\hline $\begin{array}{l}\text { ELETFIOAL } \\
\text { ENEFGY USE }\end{array}$ & 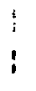 & $\begin{array}{r}\text { oftupied } \\
(\text { thwh })\end{array}$ & i & $\begin{array}{c}\text { Woocubied } \\
\text { (\$) fwh) }\end{array}$ & \\
\hline Compressor & : & 0.050 & i & 0.050 & $\mathrm{i}$ \\
\hline FeE istanEe & i & 0.050 & ! & 0.050 & \\
\hline Inductive & $!$ & 0.050 & $i$ & 0.050 & $i$ \\
\hline
\end{tabular}

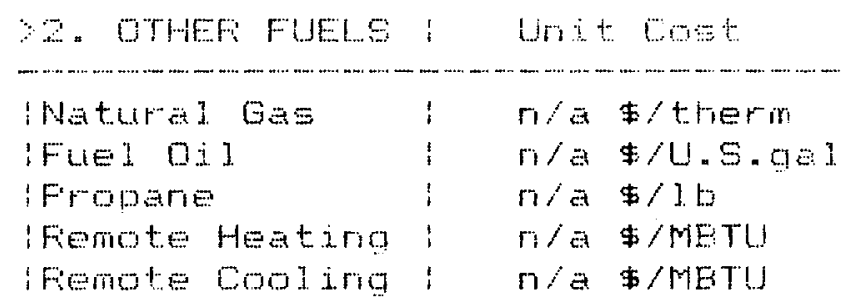


Tob Wame " D-O GUECOH $1 / 3$ DESTGN SYS

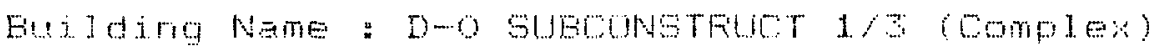

Site Name : AUFOFA, IL FEFMI, UEEr DEfined
Dete $=11-16-87$

Serial. Number: 00117862.0

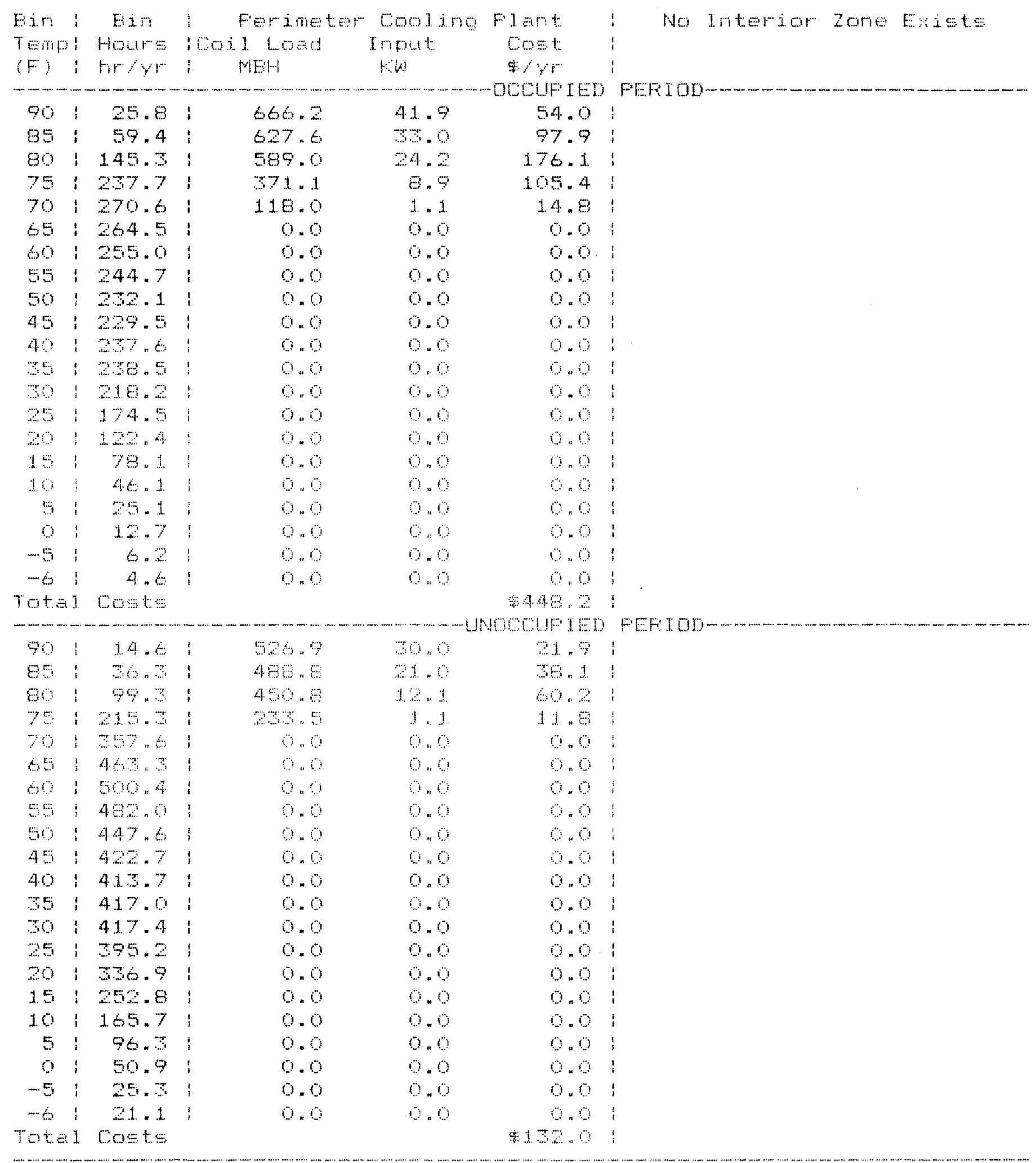




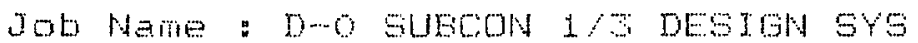

Date: $: 11-16-87$

Serial Number: 60117862.0

Site Name : AUropa, IL FEFrt, User Defined

No Auxiliary Flant Used

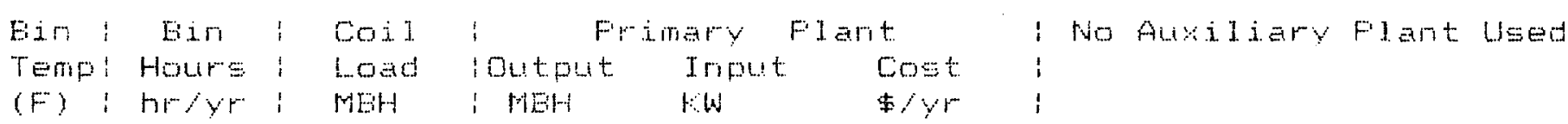

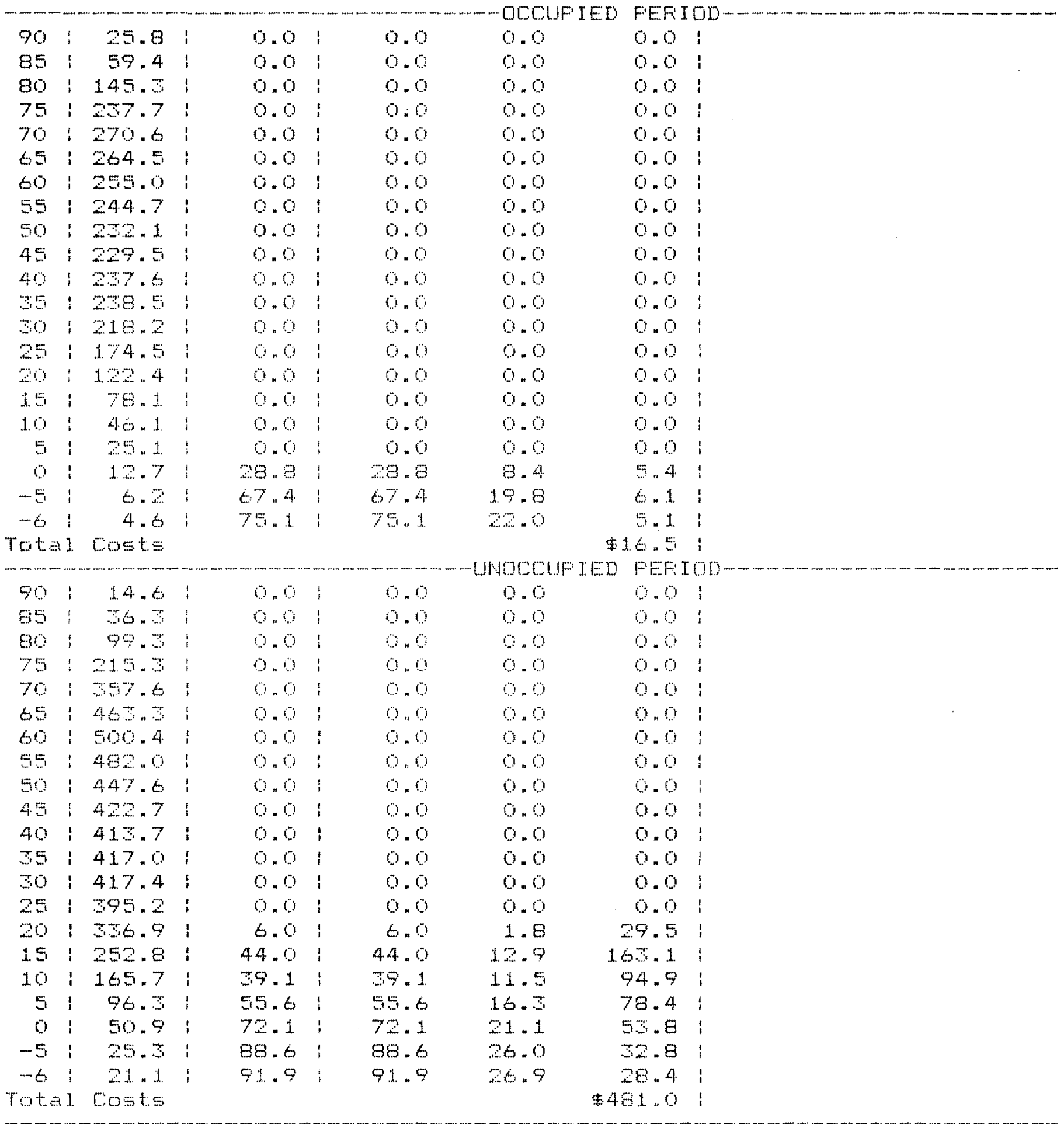


Job Name : D-O SUECON $1 / 3$ DESIGN SYS

Euilding Name: D-O SUECONGTFUCT 1/3 (Complex)

Site Name: AUFOFA, IL FEFMI, User Defined
Date: $11-16-87$

Serial Number":

60117862.0

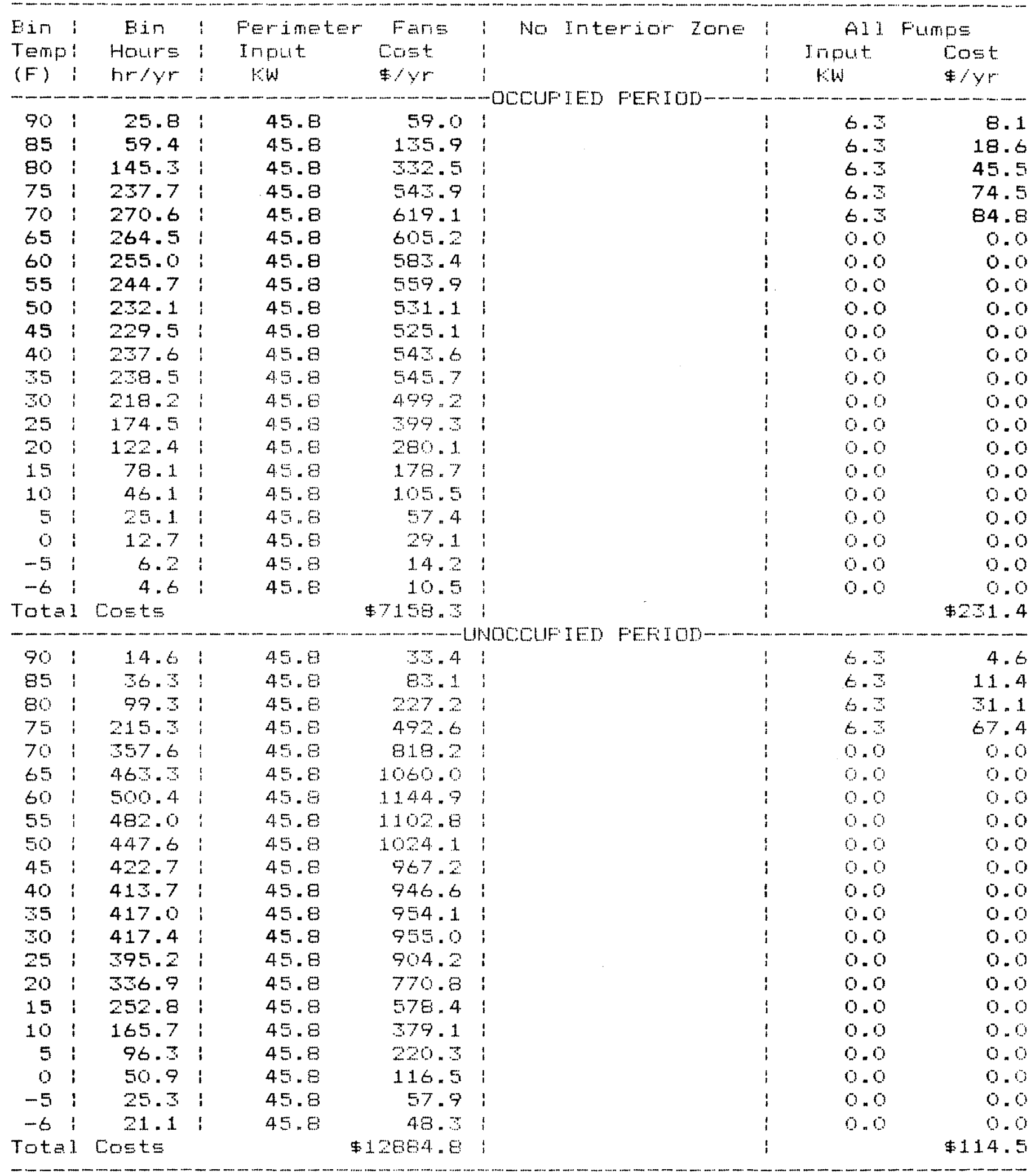




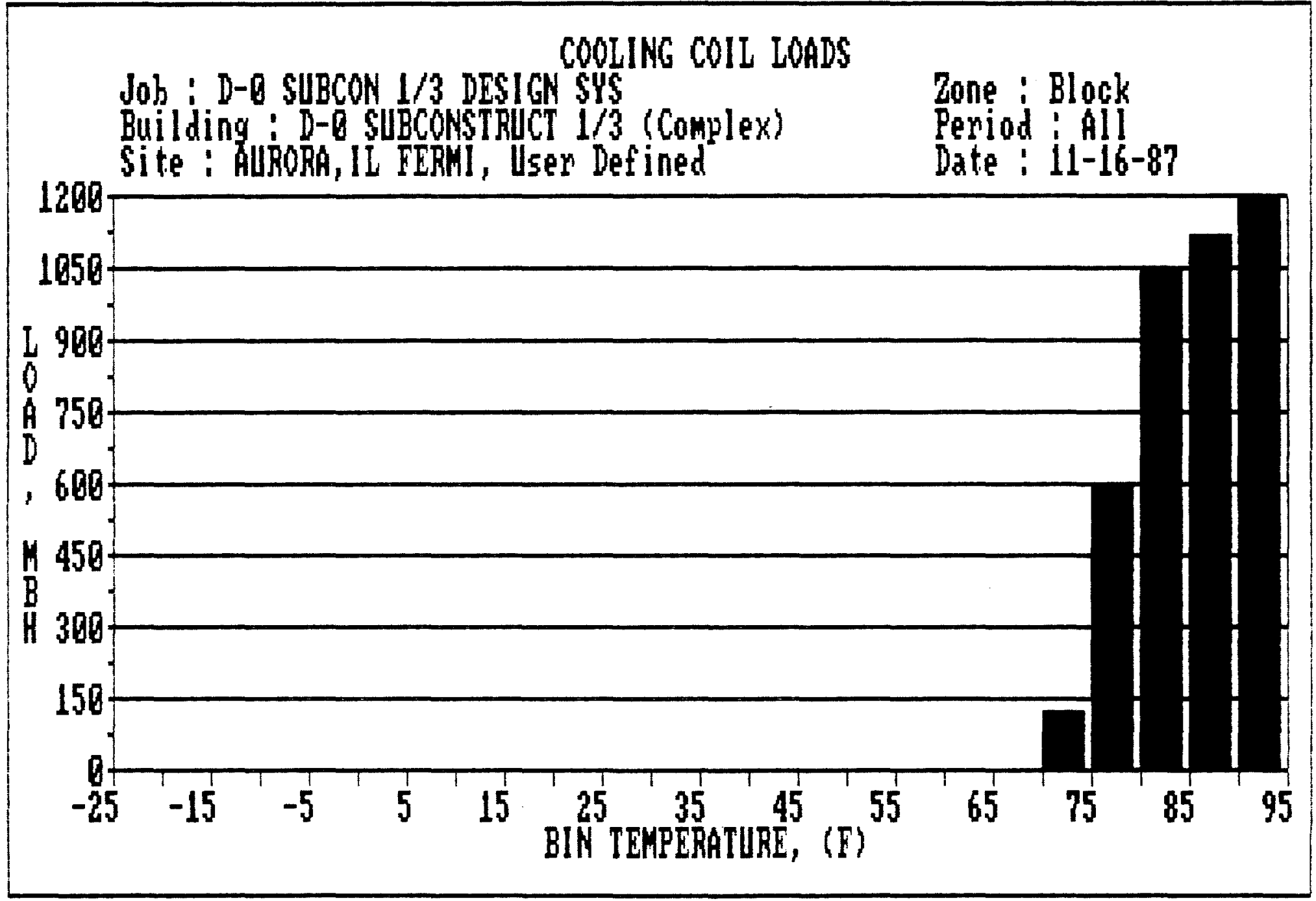




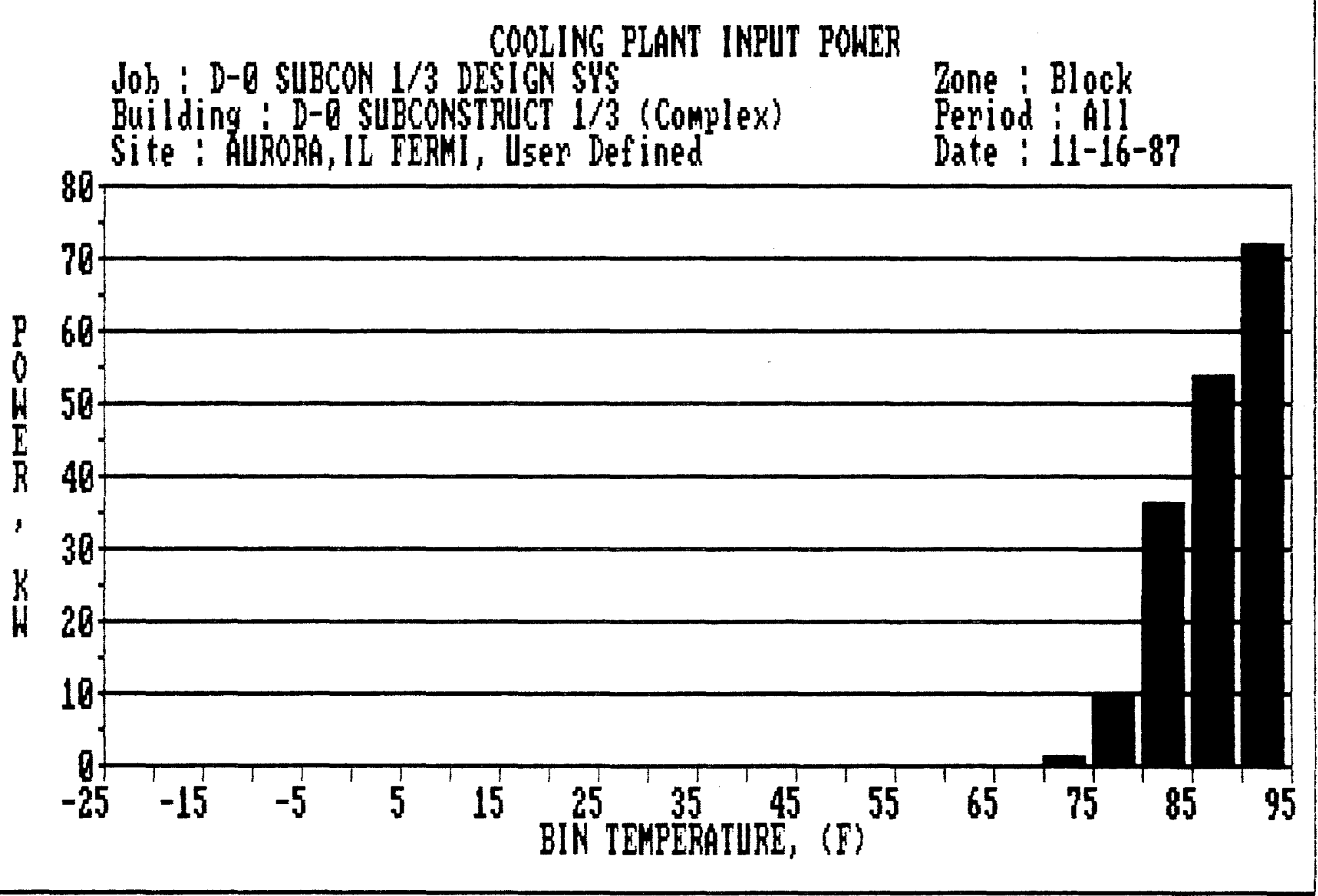




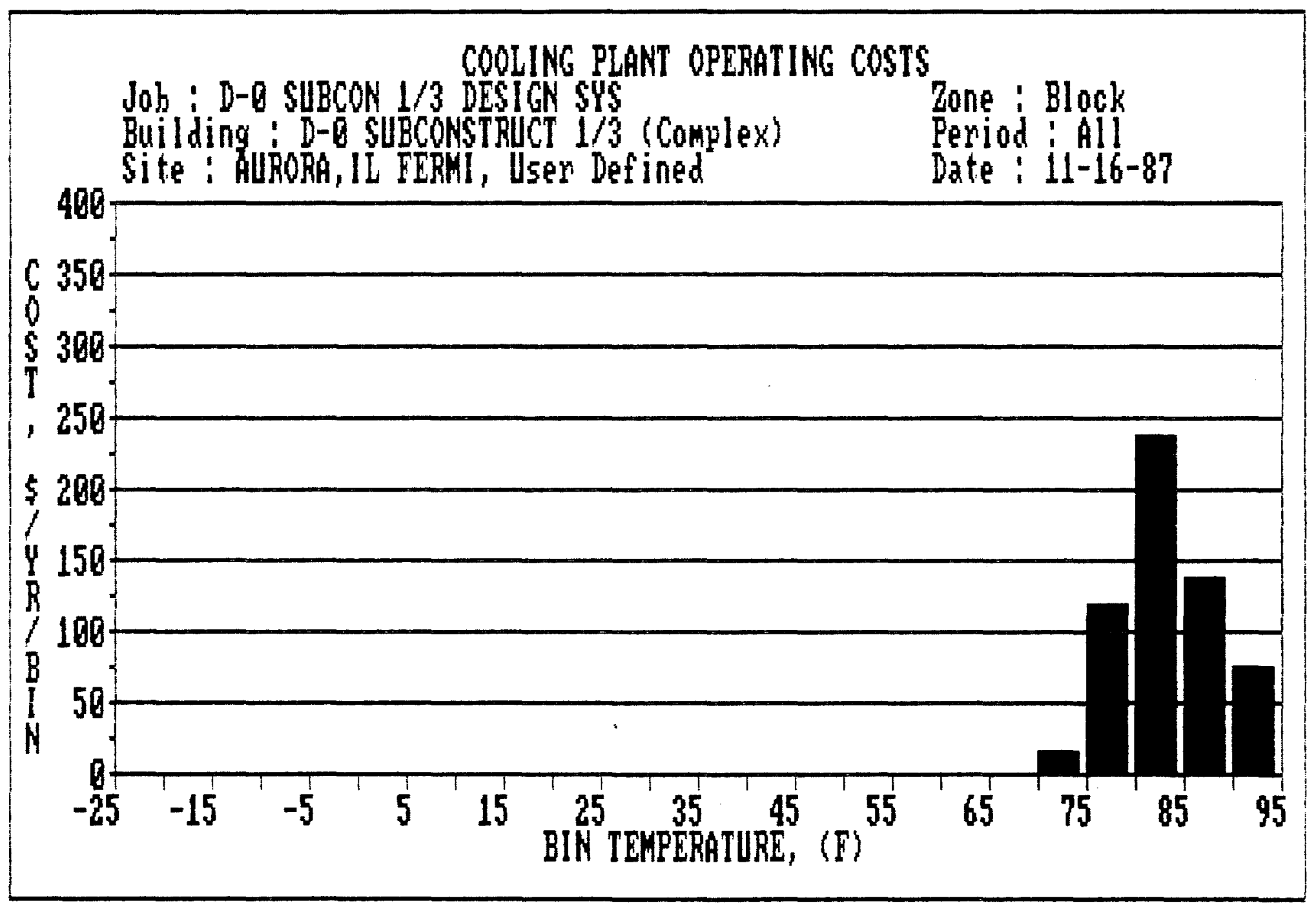




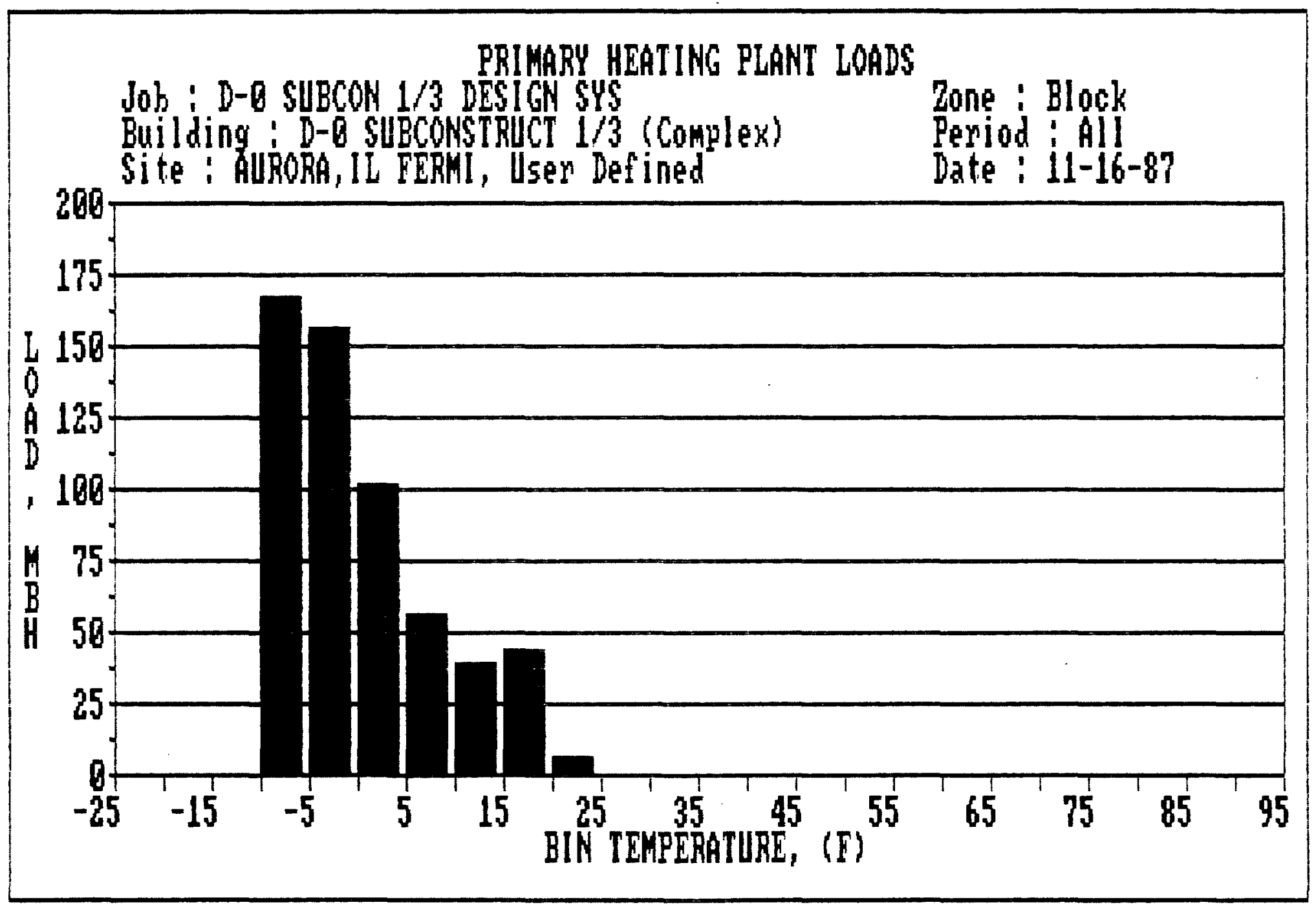




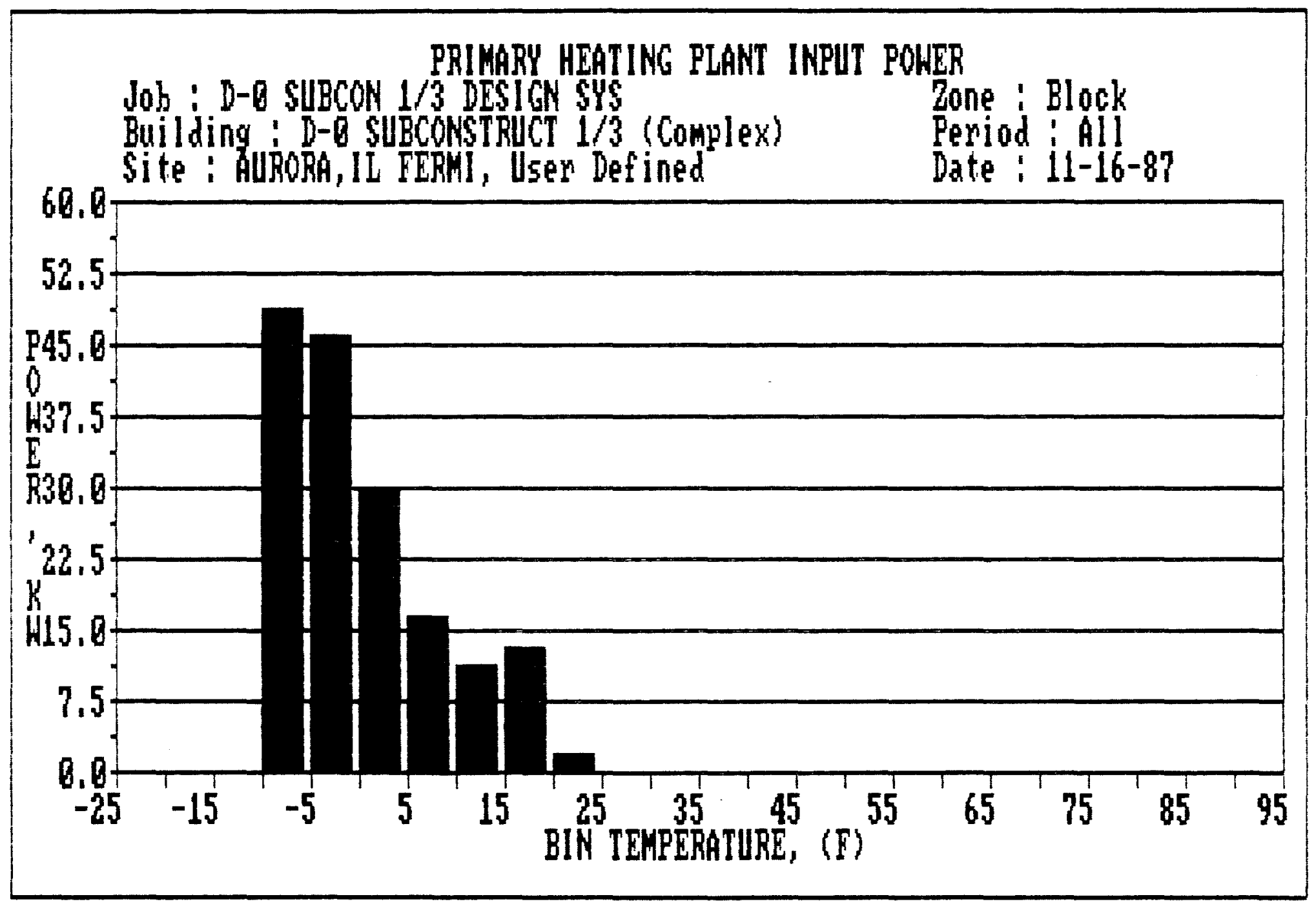




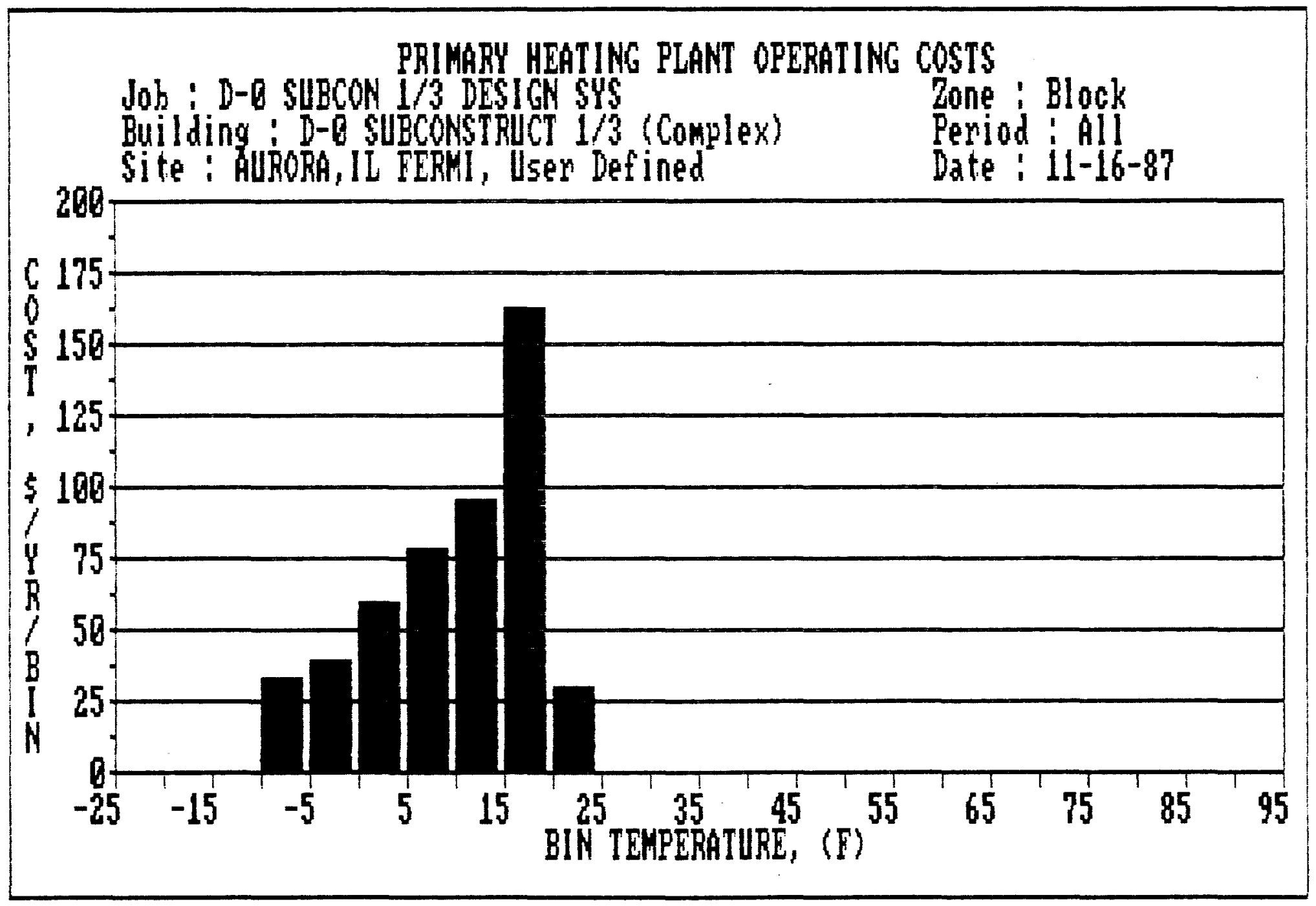




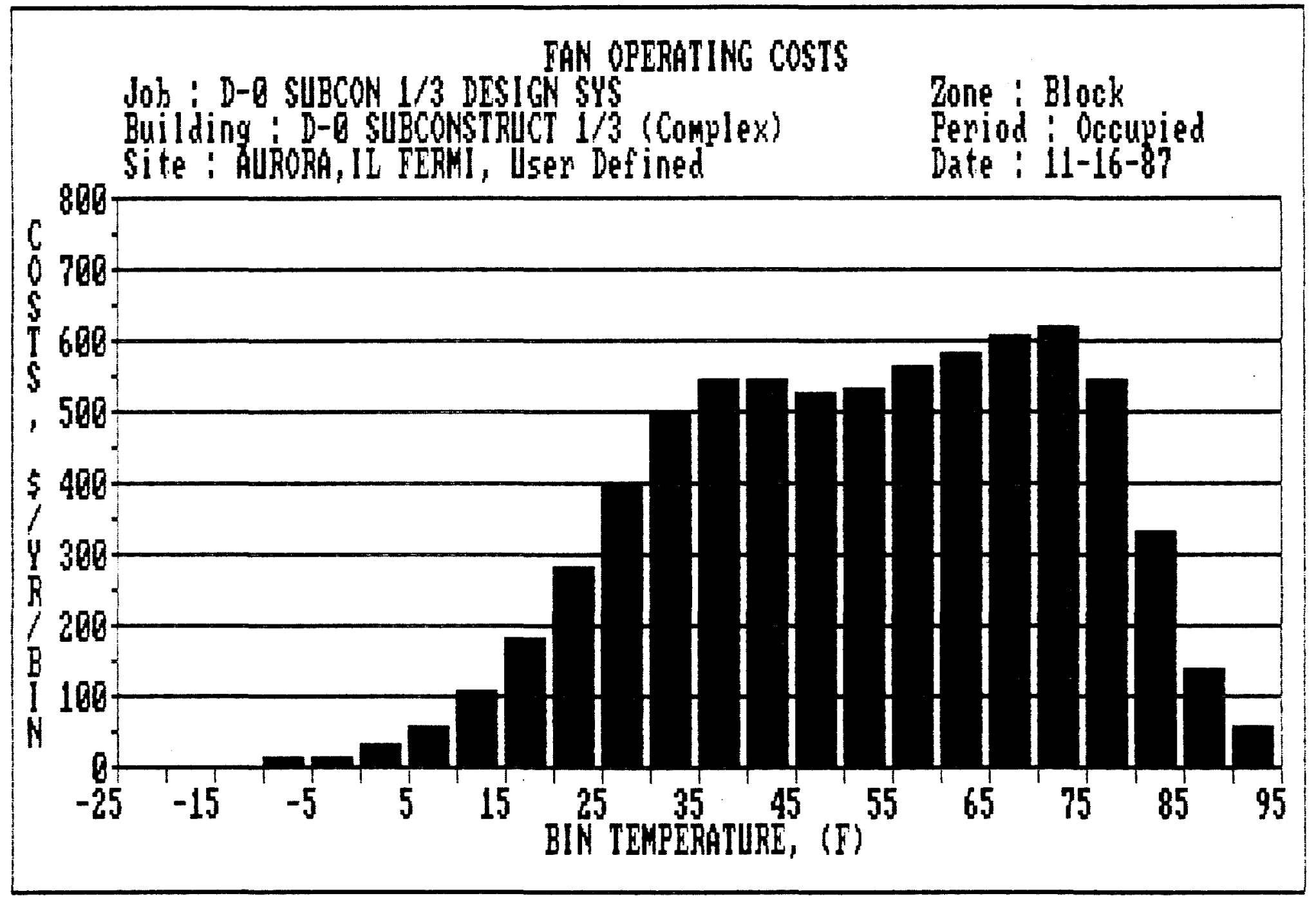




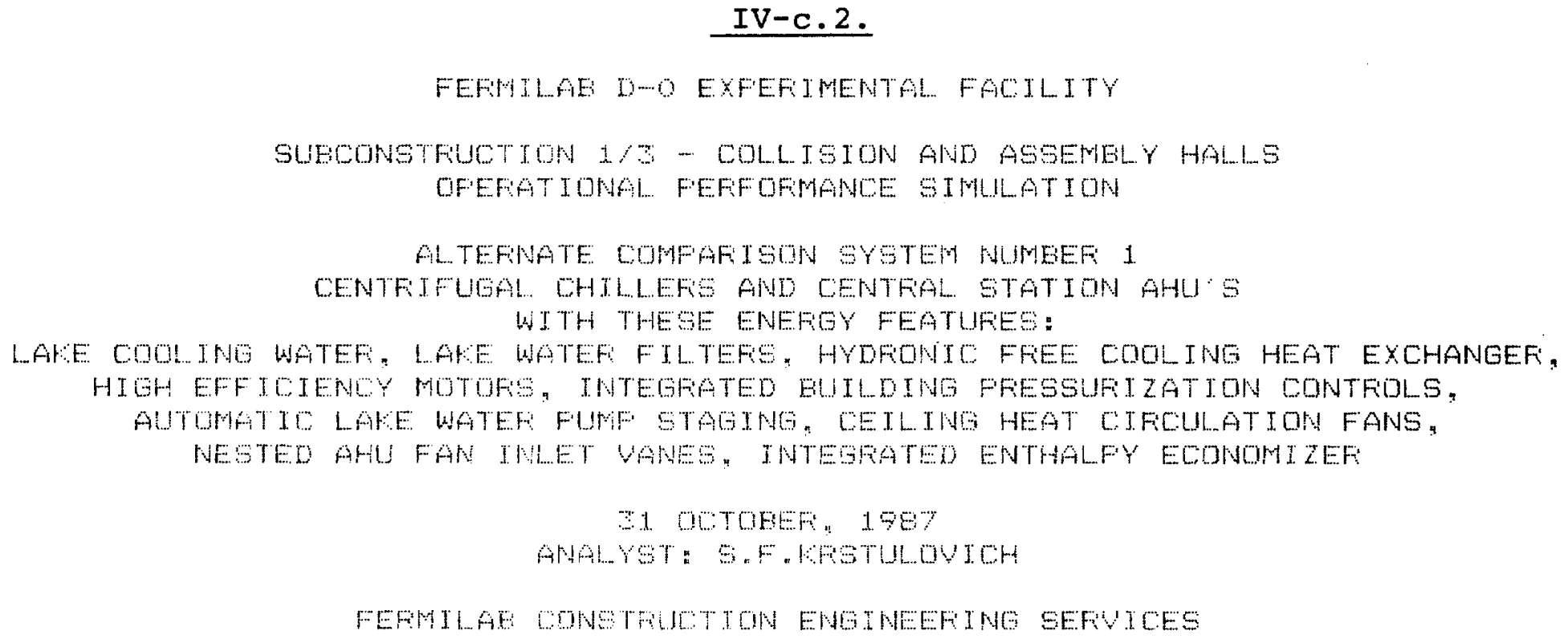




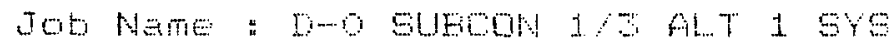

Euiddiro Nane : D-O SuECONSTFUT $1 / 3$ (Complex)

Site : AUFOFA, IL FEFHI USE Defined

Scope of Aratysi= : Cooling and Heating syeteme

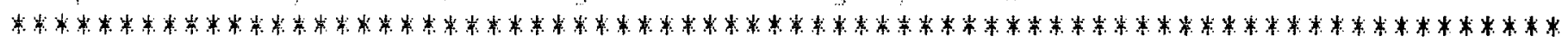

A. AIF: HANDLING SYSTEME

1. FEFIMETEF TEFMINAL TYFE : CONSTANT VOLUME

Supply fan type : Eackward inelined or airfoil with inlet vanes

Supply fan total static pressure = E.oo in. wa.

Feturn fan type : Controlled pitch axial.

Fieturn fan total static pressure $=1.75 \mathrm{in.}$. wo.

Design supply air= $42000 \mathrm{cfm}$ a $67 \mathrm{~F}$; Vent. air= $3000 \mathrm{cfm}$

Are cooling terminals used for heating? YY

Economizer type : Integrated, enthalpy controlled

Is a ventilation reclaim device used? N?

Are ventilation air dampers closed for unoccupied periods $? \mathrm{~N}$ :

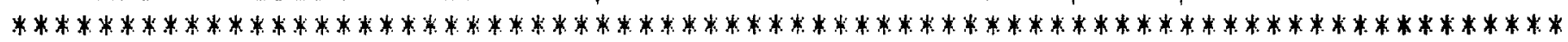

B. HEATING FLAANTS

\% 1. FEFIMETEF PLANT : ELEETFICAL FESISTANCE

Is hydronic heating used? $? \mathrm{~N}$

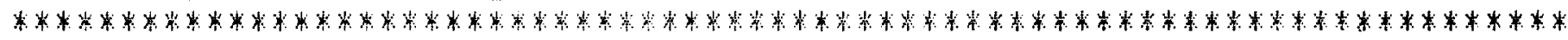


Tot Name : D-O SUEOON $1 / 3$ fl.T 1 SYS

Euilding Nane : D-O SUECONSTFUCT 1/Z (EOMPlEx)

Site : AUFOFA IL FEFWI UEE Defiried

Soope of fnelysis y Coolimg and Heating Systems

Date " $11-16-87$

601.7862 .0

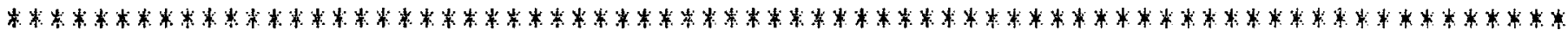

C. COOLING FLANTS

1. FEFIMETEF FLANT : CENTFTFUGAL WATEF COOLED CHILLEF

Capacity $\mathrm{g}$ S $\mathrm{F}$ entering water $=75.0$ tons

rw/Ton e $85 \mathrm{~F}$ entering water $=0.78 \mathrm{kw} / \mathrm{tor}$

Is hydronic cooling used? Yy

IE chilled water reset used $? \mathrm{~N}$

Is condenser performance altitude adjusted? ¿Y

Heat sink type: Open cooling tower

Minimum entering water temperature = $22 \mathrm{~F}$

Is a hydronic economizer used? cy ; Economizer efficiency $=80 \%$ Number of sequenced chillers $=2$

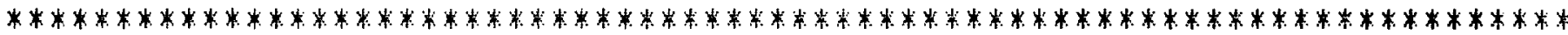

D. FUFFTHE SYTEFS

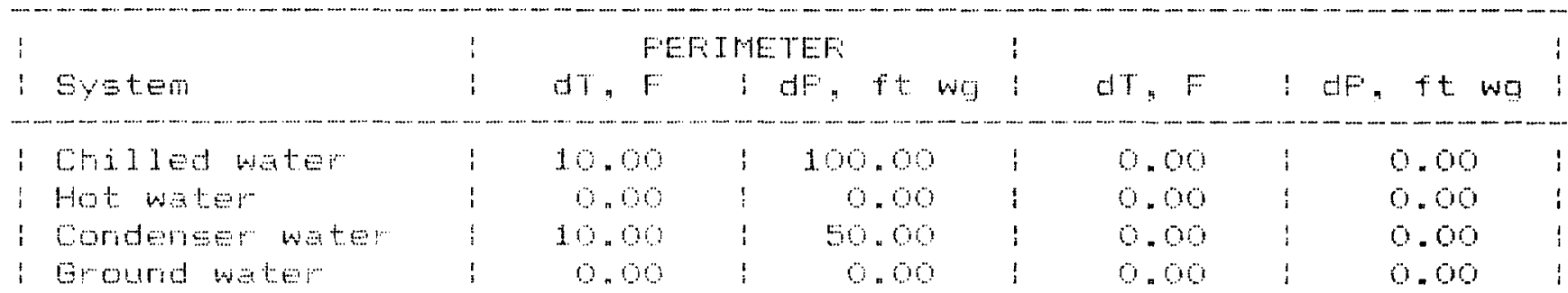

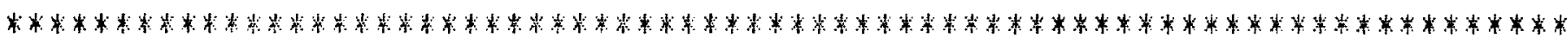

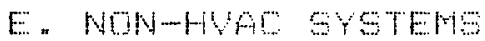

1. NOHWHAO ELETTFIOCL EVEROY USE

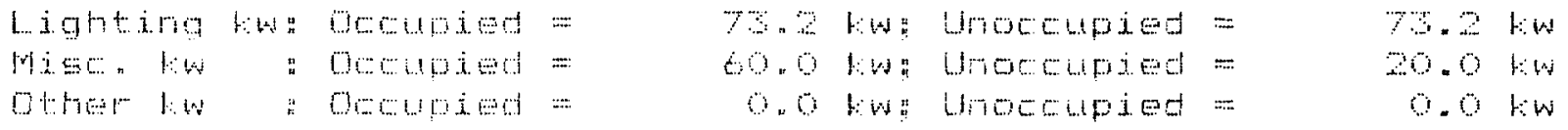

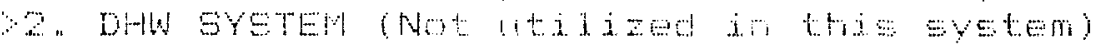

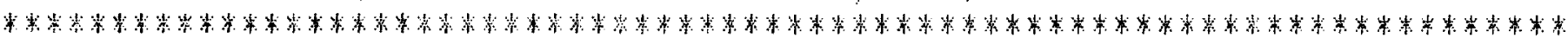
F. FUEL WOST:

\begin{tabular}{|c|c|c|c|c|c|c|c|c|}
\hline$\because 4$ & 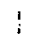 & \multicolumn{3}{|c|}{$\operatorname{Fen}+\mathrm{sed}$} & $i$ & OTHE FUELS & $\vdots$ & Whit Cost \\
\hline ELECTATIAL & $\vdots$ & $0 \ldots \cos \theta$ & $i$ & Wromotipted & $\vdots$ & 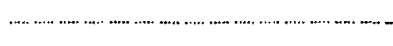 & 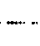 & 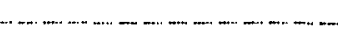 \\
\hline ENEFGY LOE & i & $(\mathrm{s} / \mathrm{tan})$ & i & 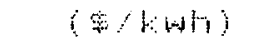 & $!$ & Hatumal $b a$ & $i$ & $r / a+t h e r m$ \\
\hline 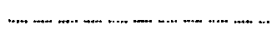 & $-\cdots$ & a.t. & $\cdots . .$. & 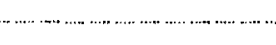 & ........ & 'wne] Oij & 1 & $\$ / \mathrm{L} .6 .901$ \\
\hline Compresssor & $i$ & 0.650 & $i$ & 0.050 & $\mathfrak{i}$ & IFroparie & $!$ & 事/ Ib \\
\hline FEsistance & 1 & 6.09 & $i$ & 6. & 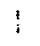 & Femote Heating & 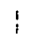 & \$/METU \\
\hline Inductive & $i$ & 0.06 & $i$ & 0.050 & $i$ & :Femote Cooling & $i$ & $n / a$ METU \\
\hline
\end{tabular}


TOTAL DUILDING EHEFGY SUMAF'

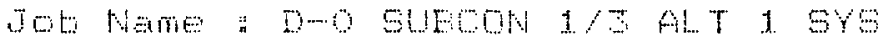

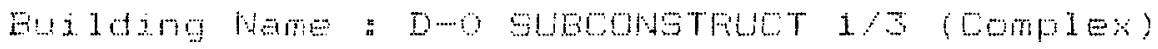

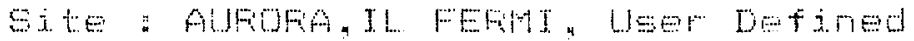
Fimiod: AII
Zone: Elocl
Deto: $11-16-87$
Serial Number:
6011706.0

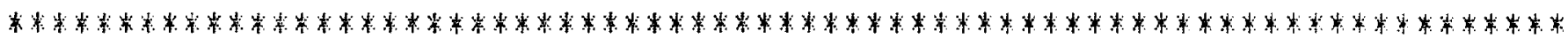

HWC ENEFGY

Ariri!n

cost

Component

Electric (oco)

Electric (Unact)

Natural Gas

Fuel. [i. 1.

Fropane

Fiemote Heating

Femote Coolino

HUAC, Total

(

7894
13624

3624

0

o

i)

())

i)

(1)

21517
Energy or Fuel

$$
\text { Limites }
$$

Gomsimed

\begin{tabular}{|c|c|}
\hline 157072 & lah/y $/ \mathrm{r}^{-}$ \\
\hline 272475 & futh $/ y+r$ \\
\hline 0 & therms $/ y r^{m}$ \\
\hline 0 & U.S. 9aj/yr \\
\hline 0 & $1 b / y r$ \\
\hline 0 & METU/YF \\
\hline 0 & METU/Yr \\
\hline
\end{tabular}

\begin{tabular}{rr} 
DUE FUF & DOE FIF \\
$(1000$ BTU) & $(1000$ ETU \\
\hline 1851320 & 538819 \\
\hline 160711 & 929958 \\
0 & 0 \\
0 & 0 \\
0 & 0 \\
0 & 0 \\
0 & 0 \\
-499201 & 1468776
\end{tabular}

NON-HYA ENEWY

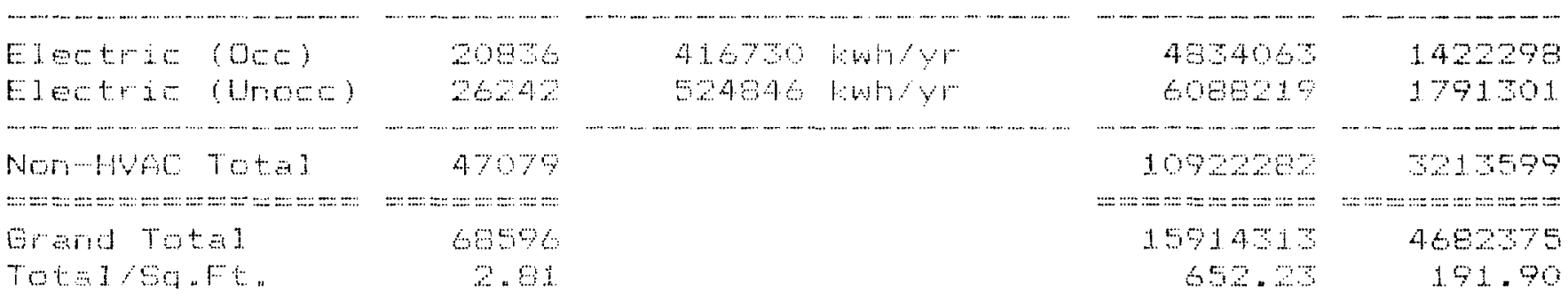

भบक्ष
HAL TOEA ROE.

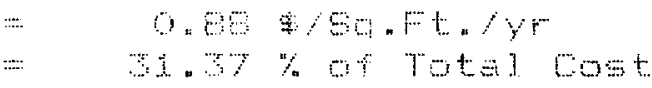
Total Hyat Elemerame Erama

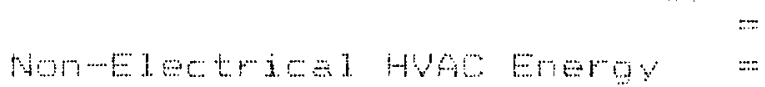

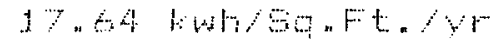

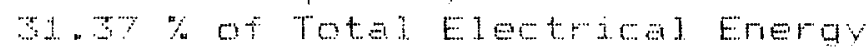
मо भ की Totol Energy

$1+1$

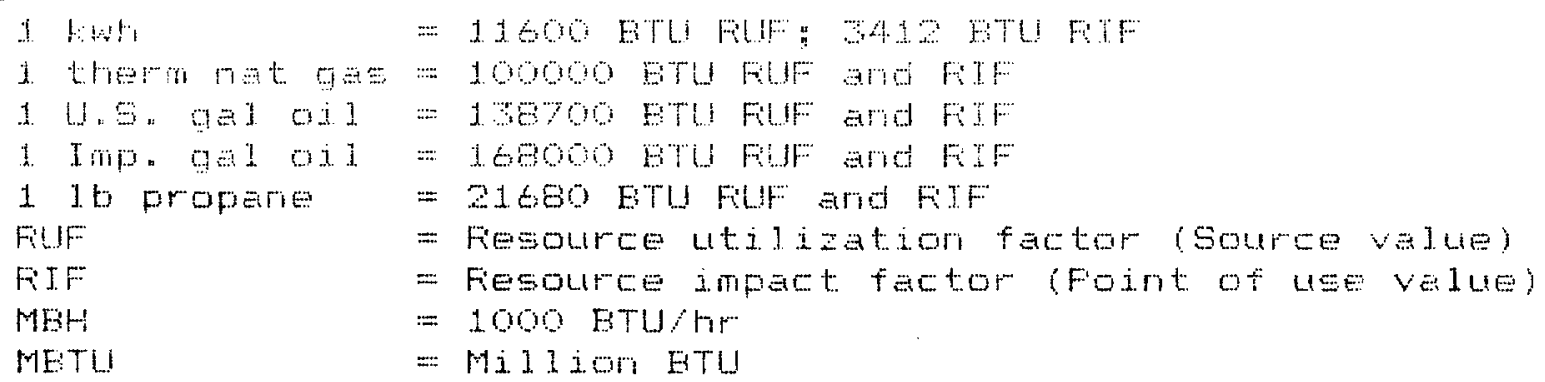




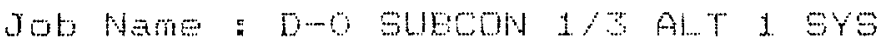

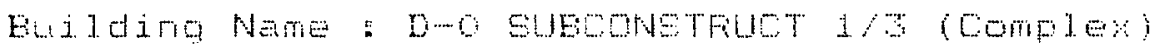

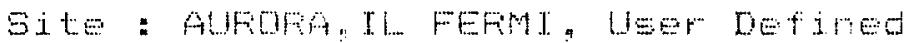

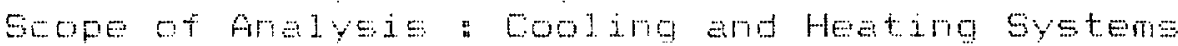

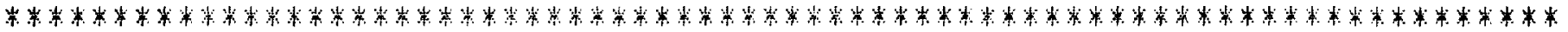

A. AJF HANDL ING SYSTERS

CANHUA FAN OFEFATMNG COST=

$\$ 0,045$ ?

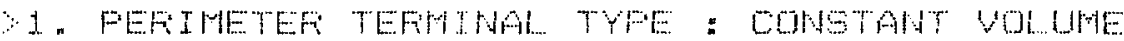

Supply tan type : Eachard incined or airfoil with inlet vanee

Supply fan total. stetic pressure $=$ Joo in. wa.

Fieturn fan type : Controlled pitch axial

Fieturn fan tota]. statio pressure $=1.75$ in. wo.

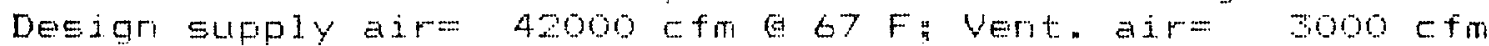

Are cooling termina]s used for heating $?$ YY

Economizer type : Integrated, enthalpy controlied

Is a ventilation reciaim device used $?$ in

Are ventilation air dampers closed for unoccupied periods ? cN?

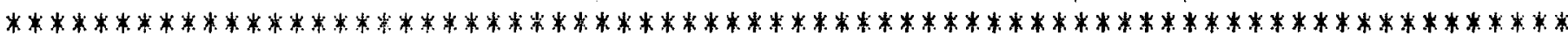

E. HEATING FLANTS (ANUUAL HEATING FLANT OFEFATING COST =

Y 1. FEFTMETEF FLANT \# ELECTFIOAL FESISTANOE

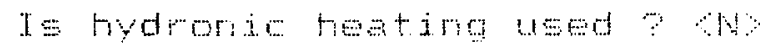

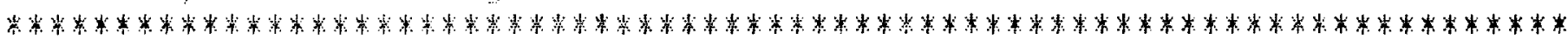


Job Name : D-O SUECON $1 / \mathrm{ALT}$ I SYS

Fige 2

Building Name : D-O EUECONSTFUCT $1 / 3$ (Complex)

Date: $: 11-16-87$

60117862.0

Site a AUFOFA II. FEFMT, USer Defined

Scope of Analysis a Cooling and Heating Systems

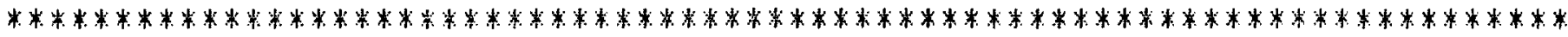

C. EOULING FLANTS IANHUAL EOOLINO FLANT OFEFATING COST

\$8)

1. FEFIMETEF FLANT : EENTFIFUBAL WATEF COOLED CHILLEF

Capacity a $85 \mathrm{~F}$ entering water $=75.0$ tons

HW/Ton e $85 \mathrm{~F}$ entering water $=0.78 \mathrm{kw} / \mathrm{ton}$

Is hydronic cooling lised ? Y?

Is chilled water reset used ? Ny

Is condenser performance al titude adjusted ? Yे

Heat sink type : open cooling tower

Minimum entering water temperature $=32 \mathrm{~F}$

Is a hydronic economizer used? CY ; Economizer efficiency $=80 \%$

Number of sequenced chililers $=2$

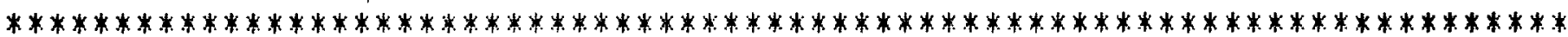

D. FUMFING SYSTEMS

(ANNUAL FUMF DFEFATINE COST =

$\$ 594)$

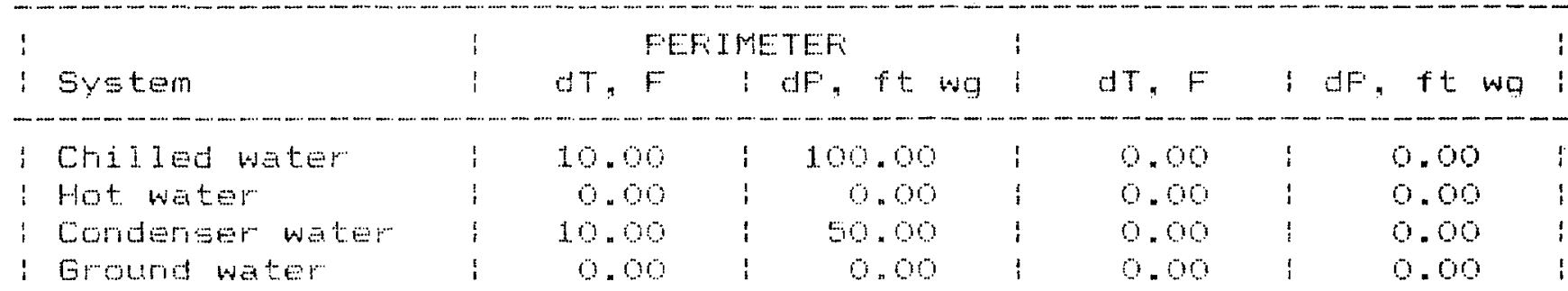

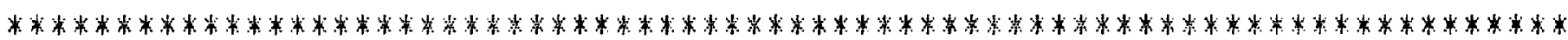

E. NON-HYAC SYSTETIS

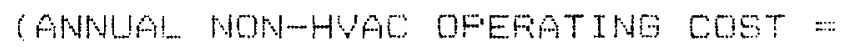

$+47,079$ )

1. NON-HVAC ELEOTFIOAL ENEFGY USE
Lighting kw: Oocupied =

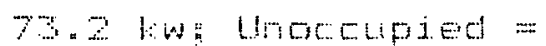
$7 \times 21: 6$
Mis.

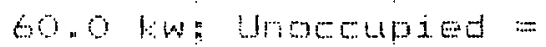
o. twe UMOCEtipied =
$20,0 \mathrm{tw}$
Other las : OCELipied =

2. DHW SYSTEM (Not litilized in this systen)

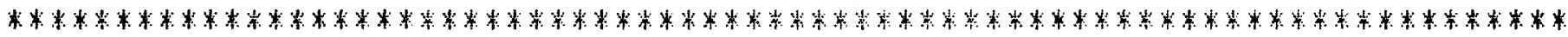
F. FUEL COETS

GAMD TOTAL AHHAL DFEFATING COST =

$\$ 68.596$ )

\begin{tabular}{|c|c|c|c|c|}
\hline 1 & 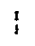 & \multicolumn{3}{|c|}{ Period } \\
\hline ELECTFICAL & i & BEEL IEd & $i$ & Uriocoupded \\
\hline ENEFGY USE & 1 & (s/lowlo) & $!$ & (t) $(4 \omega)$ \\
\hline Compressor & ! & 0.050 & $i$ & 0.000 \\
\hline Fiesistance & $i$ & 0.050 & $\vdots$ & 0.050 \\
\hline Induictive & 1 & 0.050 & ! & 0.050 \\
\hline
\end{tabular}

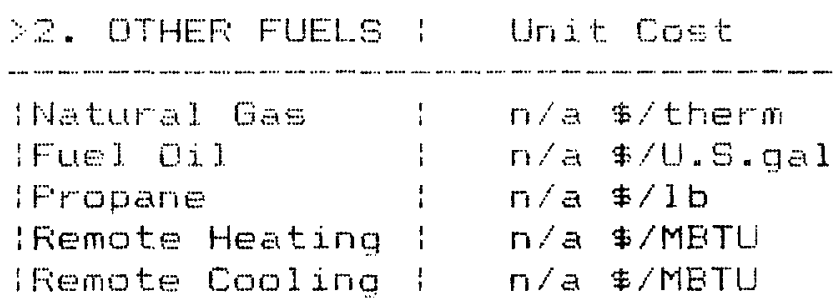




\begin{tabular}{|c|c|c|c|c|c|c|c|}
\hline Ein: & i & Ein & i & Fer ineter & Eom 1 ing & $F^{\prime} 1$ ant & No Interior Zore Exjsts \\
\hline Temp: & & Hour $=$ & {$[\mathrm{C}, \mathrm{i}]$} & I Loat & Input & $50=t$ & 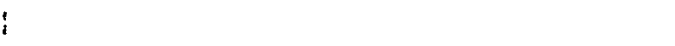 \\
\hline$(F)$ & $\mathbf{i}$ & Hiritr & $i \quad r$ & WEFI & rol & syr & 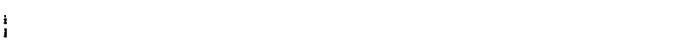 \\
\hline 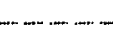 & - & 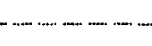 & 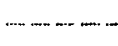 & 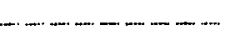 & 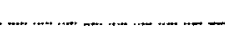 & $-0004 F]=0$ & FEFIOD - - D - \\
\hline $90 i$ & i & 25.8 & ! & 666.2 & 40.2 & 51.8 & i \\
\hline 85 & ! & 57.4 & i & 627.6 & $\because 4.9$ & 103.7 & ! \\
\hline 80 & $i$ & 145.3 & : & 589.0 & 22.2 & 161.2 & ! \\
\hline 75 & $!$ & 237.7 & i & 371.1 & 10.4 & 123.7 & $i$ \\
\hline 70 & i & 270.6 & $i$ & 118.0 & 1.1 & 14.6 & $\vdots$ \\
\hline 65 & 3 & 264.5 & 1 & 0.0 & 0.0 & 0.0 & 1 \\
\hline 60 & $i$ & 255.0 & $i$ & 0.0 & 0.0 & 0.0 & $i$ \\
\hline 55 & 1 & 244.7 & $i$ & 0.0 & 0.0 & 0.0 & : \\
\hline 50 & i & 232.1 & $i$ & 0.0 & 0.0 & 0.0 & $i$ \\
\hline 45 & 1 & 297.5 & 1 & 0.0 & 0.0 & $0 \times 0$ & $!$ \\
\hline 40 & $!$ & 277.6 & i & 0.0 & 0.0 & \% & i \\
\hline 35 & ! & 230.5 & 1 & 0.0 & 0.0 & 0.0 & $!$ \\
\hline $\mathrm{HC}$ & $i$ & 218,7 & $i$ & 9.0 & $\mathrm{O}$ & 0.0 & $i$ \\
\hline 25 & $i$ & 174.6 & 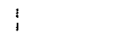 & 0 & 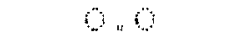 & $3: 0$ & $i$ \\
\hline 20 & $\vdots$ & $192=4$ & $i$ & i. & 0,0 & $\mathrm{O}$ & $\stackrel{3}{i}$ \\
\hline 15 & $i$ & 70.1 & $\vdots$ & 0.0 & 0.3 & ओ & i \\
\hline 10 & i & 46.1 & $i$ & ओ & ). & 1. & $i$ \\
\hline 5 & $i$ & 25.1 & $\vdots$ & 1 & a & 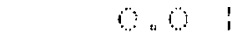 & i \\
\hline 0 & $!$ & 12.7 & $\vdots$ & $\theta=0$ & 0. & 0.0 & $!$ \\
\hline-5 & $i$ & 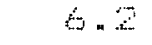 & $i$ & $\mathrm{o}^{\circ}$ & 1 & 0 & $i$ \\
\hline-6 & $!$ & 4. & 1 & औ & $\mathrm{a}$ & 0.0 & $\vdots$ \\
\hline Tot & 1. & 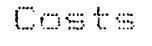 & & & & 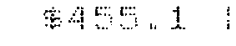 & $i^{-}$ \\
\hline …………… & $\cdots \cdots$ & 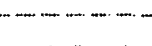 & 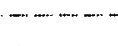 & 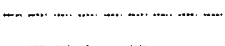 & 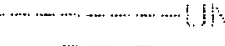 & 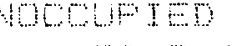 & 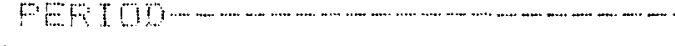 \\
\hline 90 & $\vdots$ & 14.6 & $\dot{3}$ & $86 n$ & $\because 7,7$ & धथ & 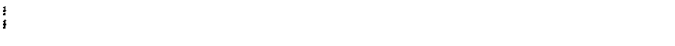 \\
\hline$\theta$ & $i$ & 76, & $!$ & $40 \mathrm{\theta}$ & 19 & 3 & $\vdots$ \\
\hline 80 & $i$ & $99 . \cdots$ & $!$ & 45,6 & $\therefore 4$ & , & $\vdots$ \\
\hline 75 & $!$ & 21,3 & 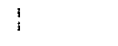 & ar 6 & 1.1 & 11,6 & ; \\
\hline 7 & 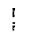 & 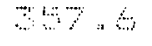 & $\vdots$ & 0 & " & ओ. & 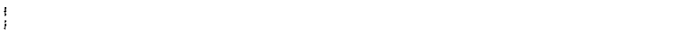 \\
\hline 5 & $\vdots$ & $46 \times$ & i & 0 & " & 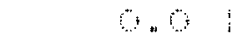 & 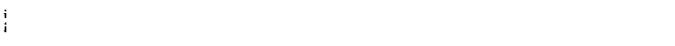 \\
\hline 6 & $i$ & 50. & $\vdots$ & औ" & a & ब & $!$ \\
\hline : & $i$ & $48 \cdots$ & $\vdots$ & ओ & i. & i, & ! \\
\hline 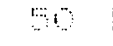 & $\vdots$ & 447.6 & $i$ & i" & $\because \because$ & 0.0 & i \\
\hline 45 & $!$ & $42=7$ & $i$ & $\theta .0$ & i & 0 & 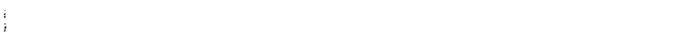 \\
\hline 40 & $!$ & $413=7$ & $!$ & 0 & 0.0 & 0.0 & $\vdots$ \\
\hline \pm 5 & $\vdots$ & 417.0 & $\vdots$ & 0.0 & 0.1 & 0.0 & 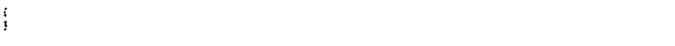 \\
\hline$\because 0$ & 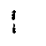 & 417.4 & $i$ & 9.0 & 0.0 & 0.0 & $\vdots$ \\
\hline 25 & 1 & 495.2 & 1 & 0 & 0 & 0 & $\dot{i}$ \\
\hline 20 & 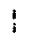 & 756.9 & 1 & 0.0 & iी & 0,0 & 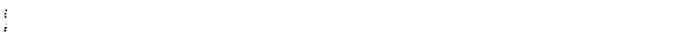 \\
\hline 15 & $i$ & 252.8 & $i$ & 0.0 & 0.0 & 0.0 & $\vdots$ \\
\hline 10 & $i$ & 165,7 & i & 0.0 & 0. & 0 & $\vdots$ \\
\hline 5 & $!$ & 96. & $i$ & 0.0 & ओ. & i. & 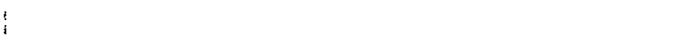 \\
\hline 6 & : & 50.9 & $i$ & 9.0 & 0 & 0.0 & ! \\
\hline-5 & 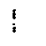 & $25, \because$ & $i$ & 0.0 & 0,0 & 0.0 & ! \\
\hline-6 & $i$ & 21.1 & $\vdots$ & 0.0 & 0 & 0. & $\vdots$ \\
\hline Tots & & $20=0$ & & & & 427,5 & $!$ \\
\hline
\end{tabular}


Job Name " $\mathrm{B}$ - SUECON $1 / 3$ ALT 1 SYS

Euijding Nene a D-o sueconetaum 1/s (complex)

Site Name : Aurarant FEmT, User Defined

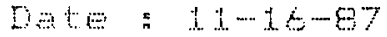

Serial Mumber :

$601179+2.0$

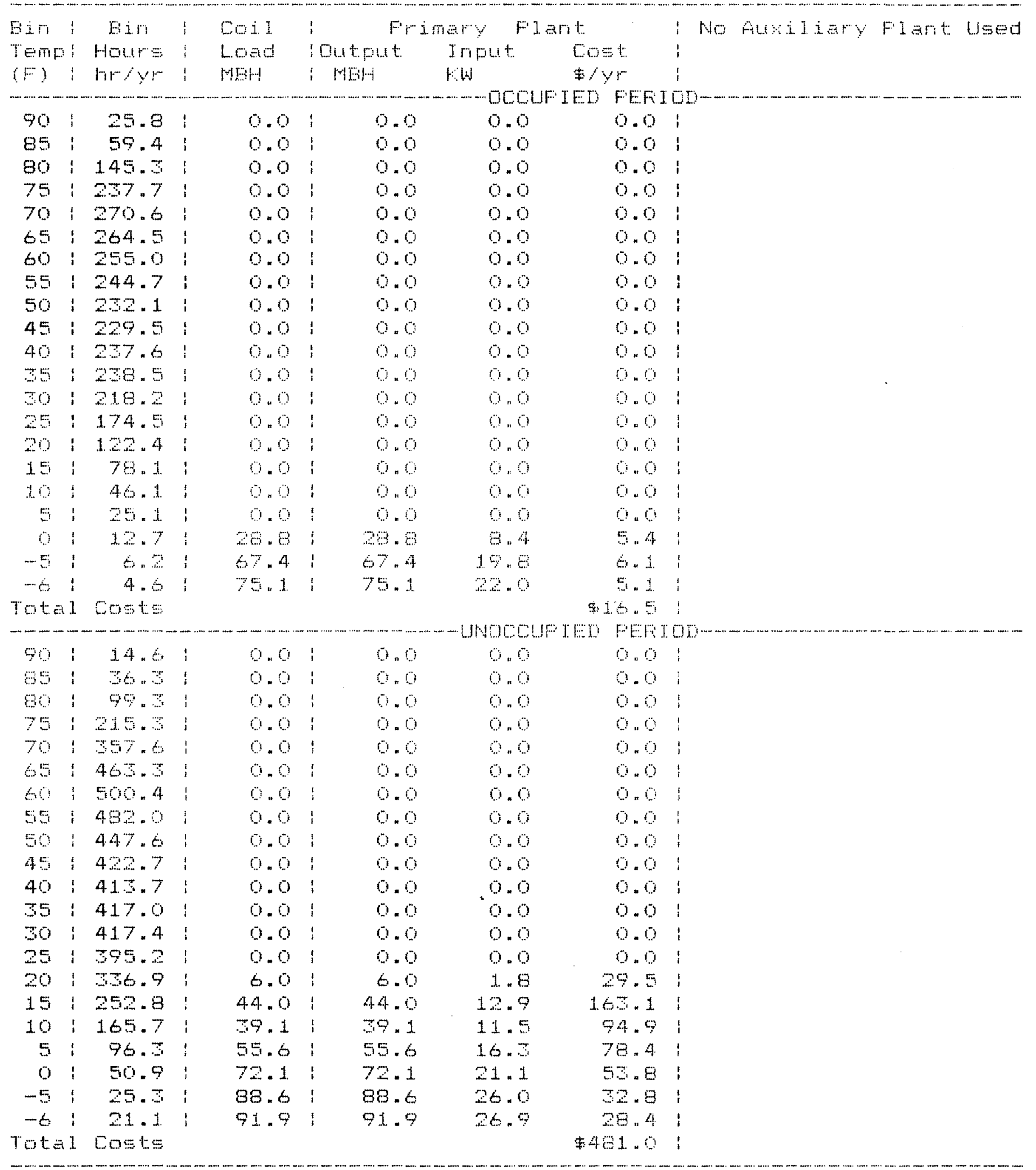


Job Name : D-O SUECOH $1 / 3$ ALT $1.5 Y S$

Buiding Name : D-O SUECONSTFuCT $1 / \mathrm{S}$ (Complex)
Date: $11-16-87$

Serial Number: 60117862.0

\begin{tabular}{|c|c|c|c|c|c|c|c|c|c|}
\hline $\begin{array}{l}\text { Ein : } \\
\text { Temp: } \\
(F):\end{array}$ & $\begin{array}{l}\text { Eirl } \\
\text { Hoirs } \\
\text { Hr/yr }\end{array}$ & $\vdots$ & $\begin{array}{l}\text { Fer imeter } \\
\text { Input } \\
\text { row }\end{array}$ & $\begin{array}{l}\text { Fams } \\
\text { Wost } \\
\text { Wyr }\end{array}$ & $\begin{array}{l}i \\
i \\
i\end{array}$ & Interior Zone & $\begin{array}{l}\vdots \\
\vdots \\
\vdots\end{array}$ & $\begin{array}{l}\text { Anput } \\
\ln \mid\end{array}$ & $\begin{array}{l}\text { Pumps } \\
\text { Cost } \\
\text { q/yr }\end{array}$ \\
\hline 90 & 25.8 & i & 45.8 & 59.0 & 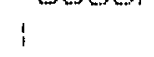 & & $i$ & 7.1 & 9.2 \\
\hline 85 & 59.4 & $i$ & 45.8 & 135.9 & $i$ & & $i$ & 7.1 & 21.2 \\
\hline 80 & 145.3 & i & 45.8 & 342.5 & 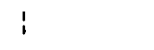 & & 1 & 7.1 & 51.9 \\
\hline 75 & 237.7 & 1 & 45.8 & 54.9 & 1 & & $\mathfrak{i}$ & 7.1 & 84.8 \\
\hline 70 & 270.6 & $i$ & 45.8 & 619.1 & $\vdots$ & & 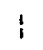 & 7.1 & 96.6 \\
\hline 65 & 264.5 & 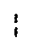 & 45.8 & 605.2 & $!$ & & 1 & 0.0 & 0.0 \\
\hline 60 & 255.0 & $i$ & 45.8 & 583.4 & $i$ & & $i$ & 0.0 & 0.0 \\
\hline 55 & 244.7 & 1 & 45.8 & 559.9 & $i$ & & $!$ & 0.0 & 0.0 \\
\hline 50 & 23.1 & 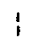 & 45.8 & $5: 1,1$ & 1 & & $i$ & 0.0 & 0.0 \\
\hline 45 & 227.5 & $i$ & 45.8 & 525.1 & $i$ & & $i$ & 0.0 & 0.0 \\
\hline 40 & $237 \cdot 6$ & 1 & 45.8 & 54.6 & $!$ & & $i$ & 0.0 & 0.0 \\
\hline 35 & 230.5 & $!$ & 45.8 & 545.7 & $\vdots$ & & 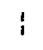 & 0.0 & 0,0 \\
\hline 30 & 218.2 & 1 & 45. & $497 \times 2$ & $!$ & & $i$ & 0.0 & 0.0 \\
\hline 25 & 174.5 & $i$ & 45.8 & 599.3 & $i$ & & $i$ & 0.0 & 0.0 \\
\hline 20 & 122.4 & i & 45.8 & 200.1 & $i$ & & $i$ & 0.0 & 0.0 \\
\hline 15 & 78.1 & i & 45.8 & 17.7 & $i$ & & 1 & 0.0 & 0.0 \\
\hline 10 & $46 \cdot 1$ & $\vdots$ & $45 \times 8$ & 100 & $!$ & & $\vdots$ & 0.0 & 0.0 \\
\hline 5 & 25,1 & $:$ & 45.6 & $5 \% .4$ & $i$ & & 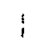 & 0.0 & 0.0 \\
\hline 01 & 12.7 & 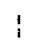 & $45 . e$ & 29.1 & i & & $\vdots$ & 0.0 & 0.0 \\
\hline-5 & 6.2 & 1 & 45,0 & 14.2 & $!$ & & $!$ & 0.0 & 0.0 \\
\hline-6 & 4.6 & $i$ & 45.3 & 10.5 & $\vdots$ & & $\vdots$ & 0.0 & 0.0 \\
\hline Total & $1 \operatorname{costs}$ & & & 9758. & $i$ & & $!$ & & $\$ 263,7$ \\
\hline 90 & 14.6 & $\vdots$ & 45.8 & $-y_{n}$ & $\begin{array}{l}\text { INOCCUI } \\
!\end{array}$ & IED FEFIOD- & $\vdots$ & 7.1 & 5.2 \\
\hline 85 & G. . & $i$ & 4.5 .8 & $8 x \times 1$ & $\vdots$ & & $\vdots$ & 7.1 & 13.0 \\
\hline 80 & 99. & $i$ & 45.8 & 227. & $\vdots$ & & $\vdots$ & 7.1 & 35.4 \\
\hline 75 & 215.8 & $t$ & 45.8 & 492.6 & $\mathrm{i}$ & & $i$ & 7.1 & 76.8 \\
\hline 70 & 7.97 .6 & $t$ & 45.6 & $\operatorname{el\theta } .2$ & 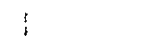 & & $i$ & 0.0 & 0.0 \\
\hline 65 & $46 \square$ & ! & 4.5 .6 & 1060.0 & $!$ & & $!$ & 0.0 & 0.0 \\
\hline 60 & 500.4 & ! & 45.6 & 1244.9 & $!$ & & $\vdots$ & 0 & 0.0 \\
\hline 55 & 482,0 & 1 & 45.8 & $1102 \cdot 9$ & 1 & & $\vdots$ & 0.0 & 0.0 \\
\hline 50 & 4.47 .6 & $i$ & 4.6 & $102+.1$ & $\vdots$ & & 1 & 0.0 & 0.0 \\
\hline 45 & 422.7 & $i$ & 45.6 & 967.2 & 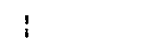 & & $i$ & 0.0 & 0.0 \\
\hline 40 & 413.7 & $i$ & 45.8 & 746.6 & $!$ & & $i$ & 0.0 & 0.0 \\
\hline 35 & 417.0 & $!$ & 45.8 & 954.1 & $!$ & & $i$ & 0.0 & 0.0 \\
\hline 20 & 417.4 & 1 & 45.6 & 955.0 & $i$ & & $i$ & 0.0 & 0.0 \\
\hline 25 & 395.2 & 1 & 45.8 & 904.2 & $i$ & & 1 & 0.0 & 0.0 \\
\hline 20 & 36.9 & $!$ & 45.8 & 770.8 & 1 & & 1 & 0.0 & 0.0 \\
\hline 15 & 252.8 & $:$ & 45.8 & 578.4 & : & & i & 0.0 & 0.0 \\
\hline 10 & 165.7 & i & 45.8 & 379.1 & $i$ & & $i$ & 0.0 & 0.0 \\
\hline 5 & 96.3 & $i$ & 4.5 .8 & 220.3 & i & & $!$ & 0.0 & 0.0 \\
\hline 0 & 50.9 & $i$ & 45.6 & 116.5 & i & & 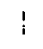 & 0.0 & 0.0 \\
\hline-5 & 25.3 & 1 & 45.8 & 57.9 & $i$ & & $\mathrm{i}$ & 0.0 & 0.0 \\
\hline$-6:$ & 21.1 & 1 & 45.8 & 48. & $i$ & & $i$ & 0.0 & 0.0 \\
\hline Total & 1 costs & & & 12804.8 & $i$ & & $i$ & & $\$ 130.4$ \\
\hline
\end{tabular}




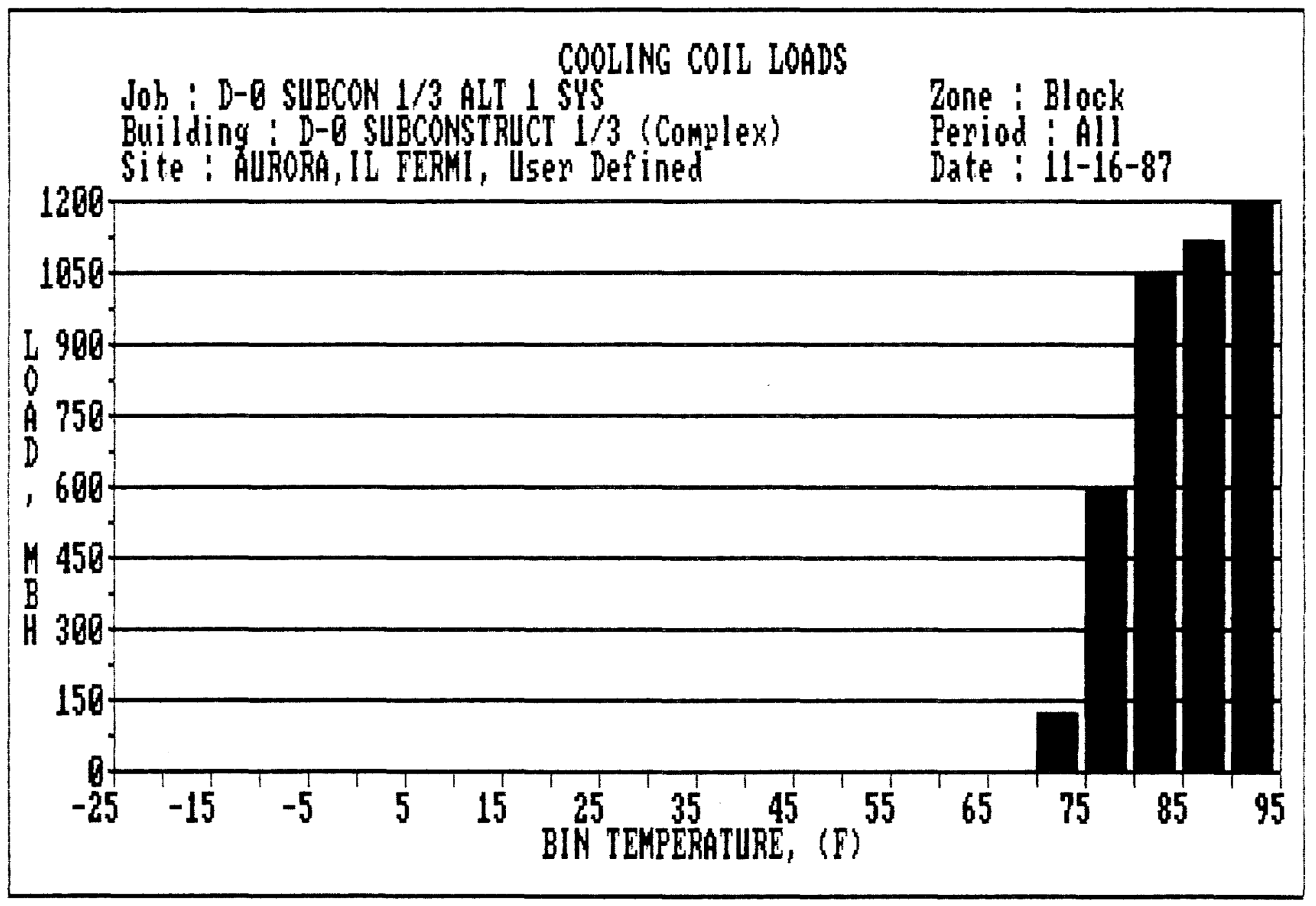




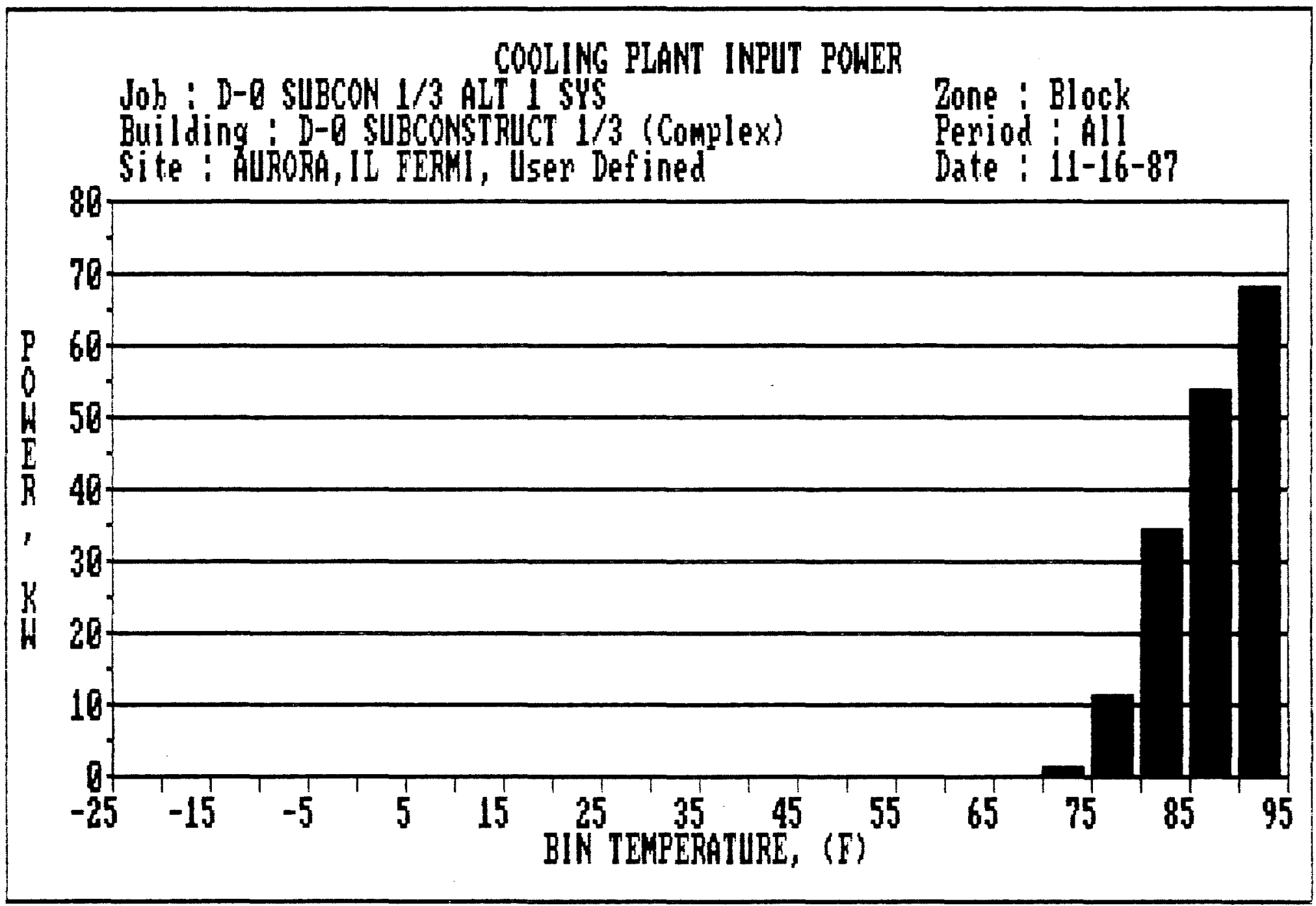




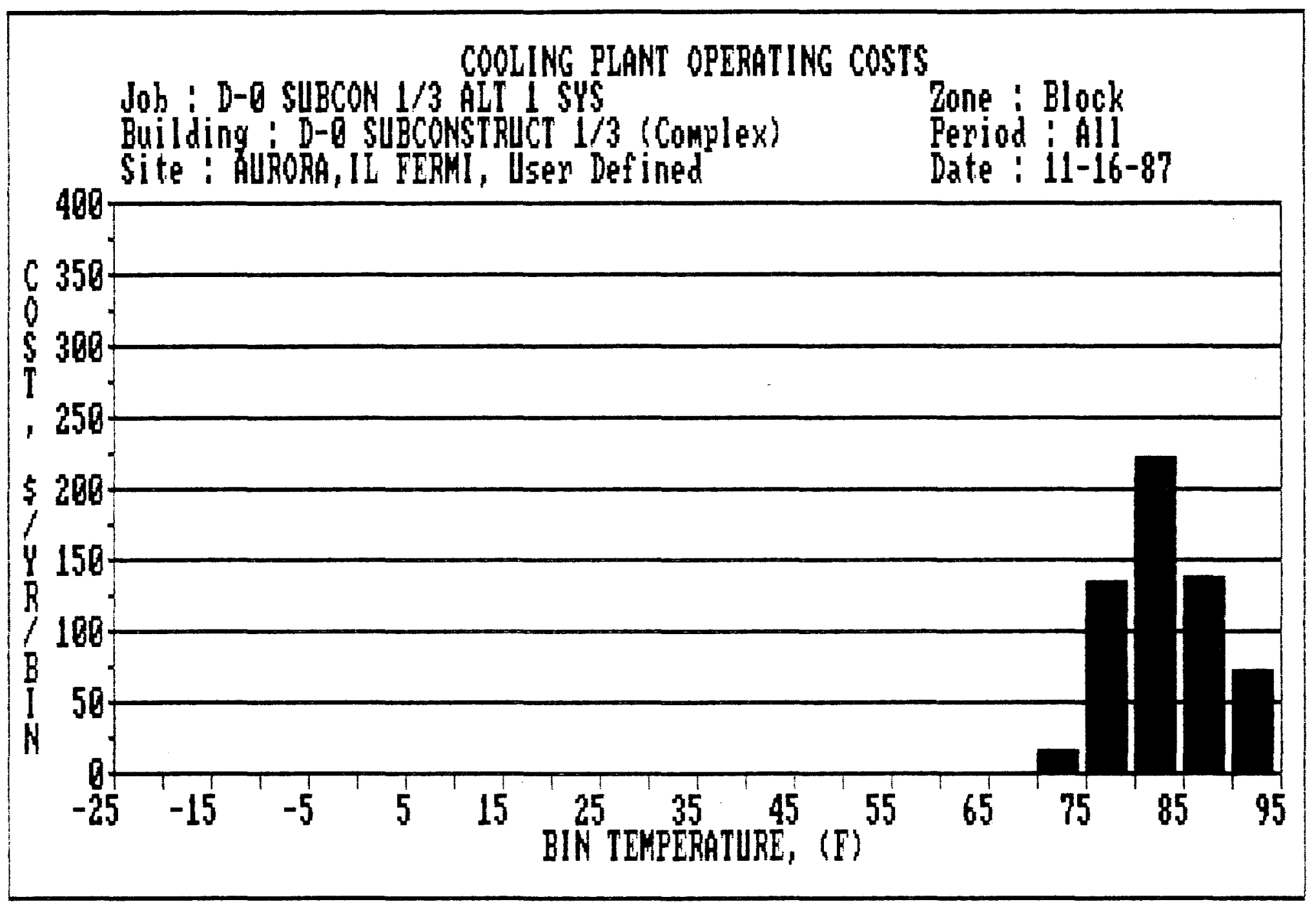




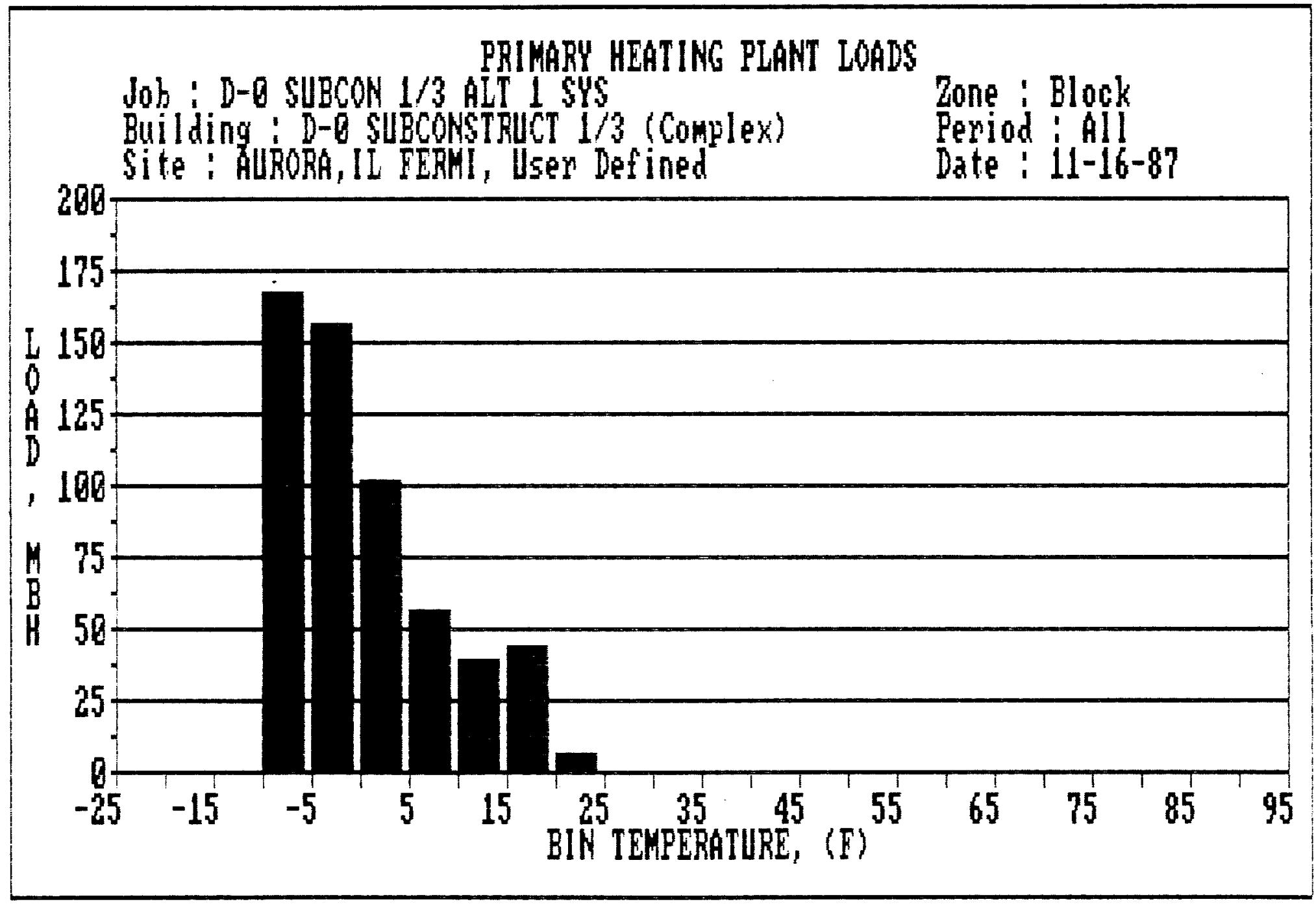




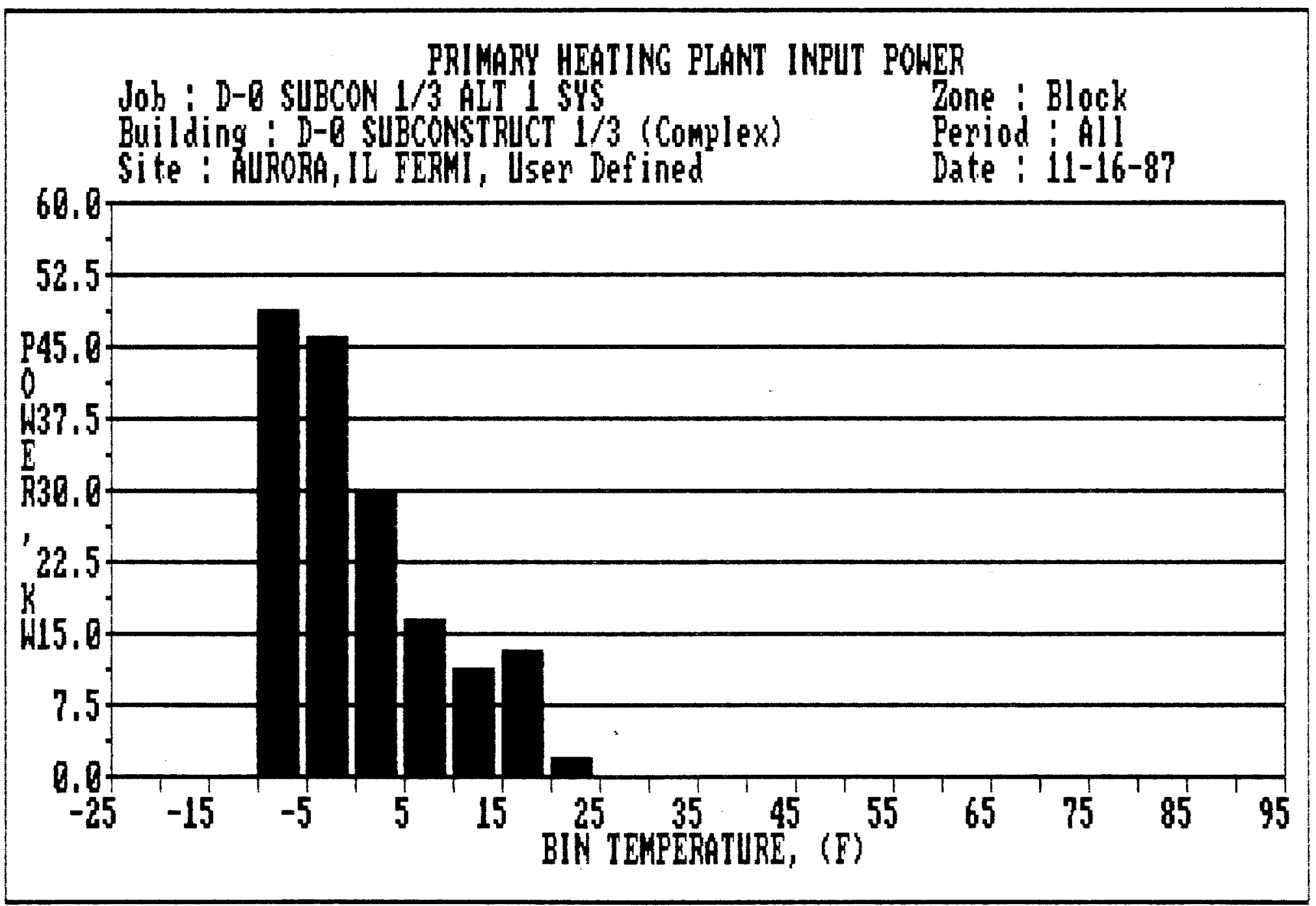




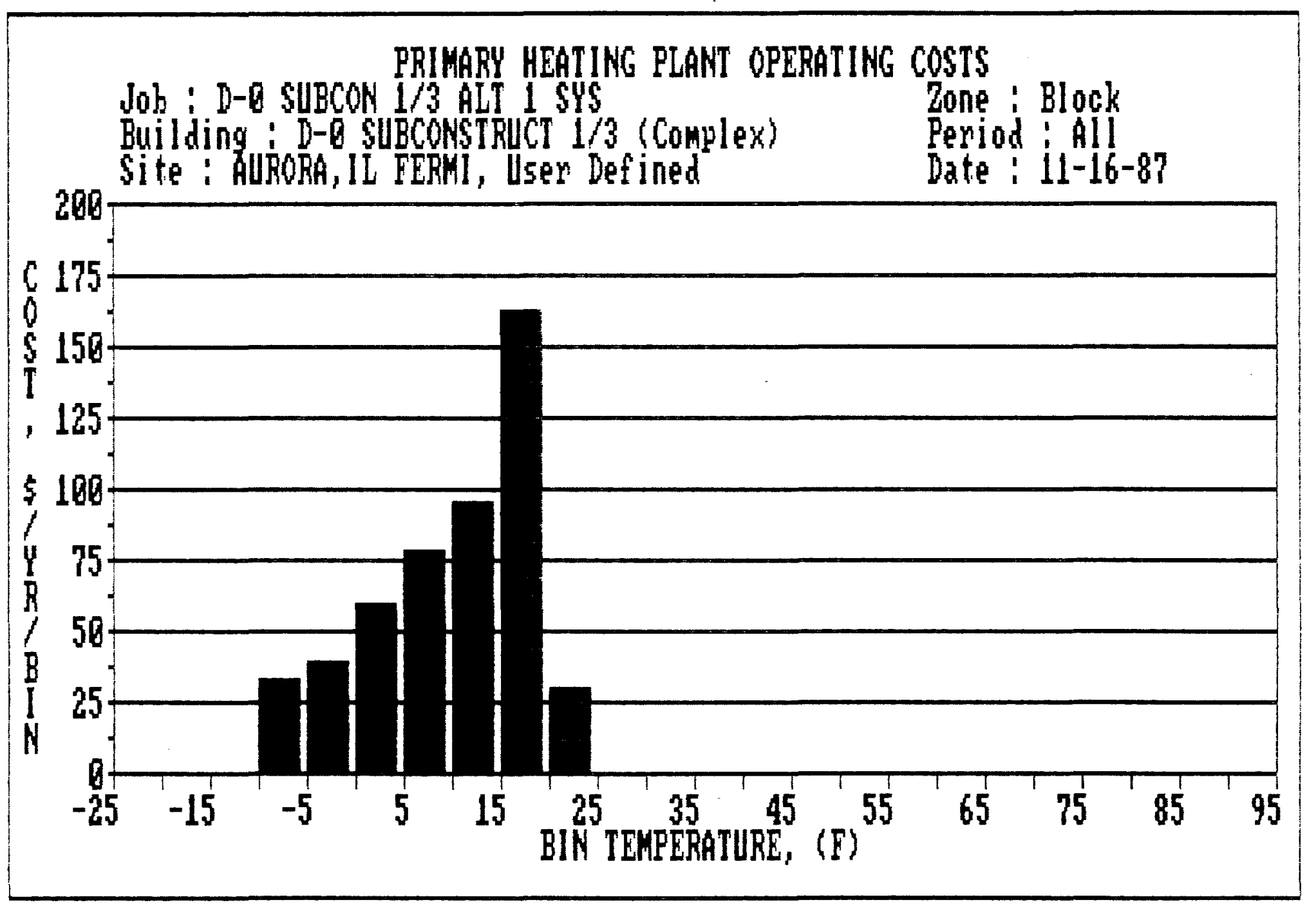


FAN OPERATING COSTS

Job: D-O SUBCON 1/3 ALT 1 SYS

Building: D-O SUBCONSTRUCT 1/3 (Complex)

Site: AlURORA, IL EERHI, Usen Def ined
Zone : Block

Period: Occupied

Date : 11-16-87

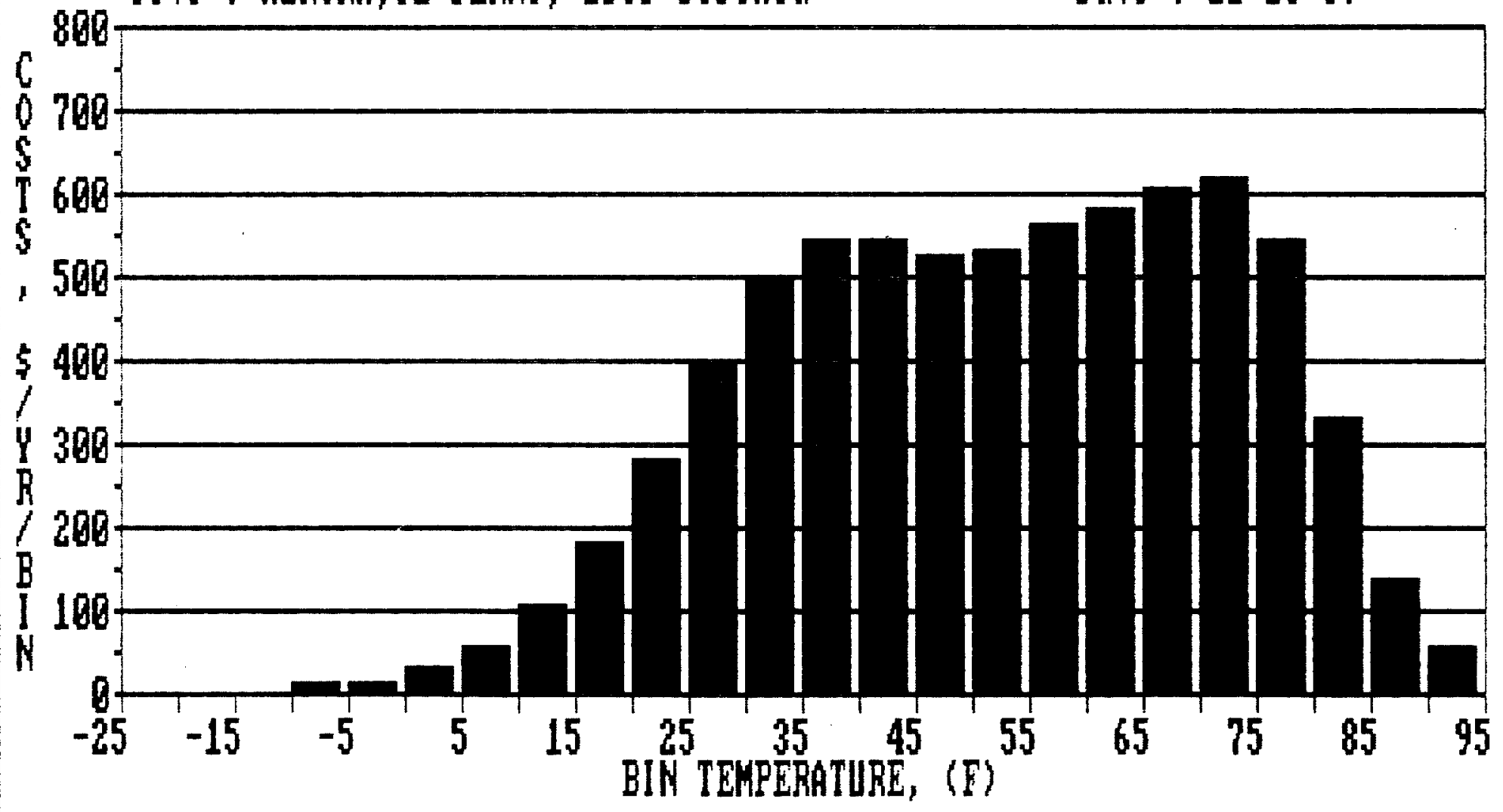




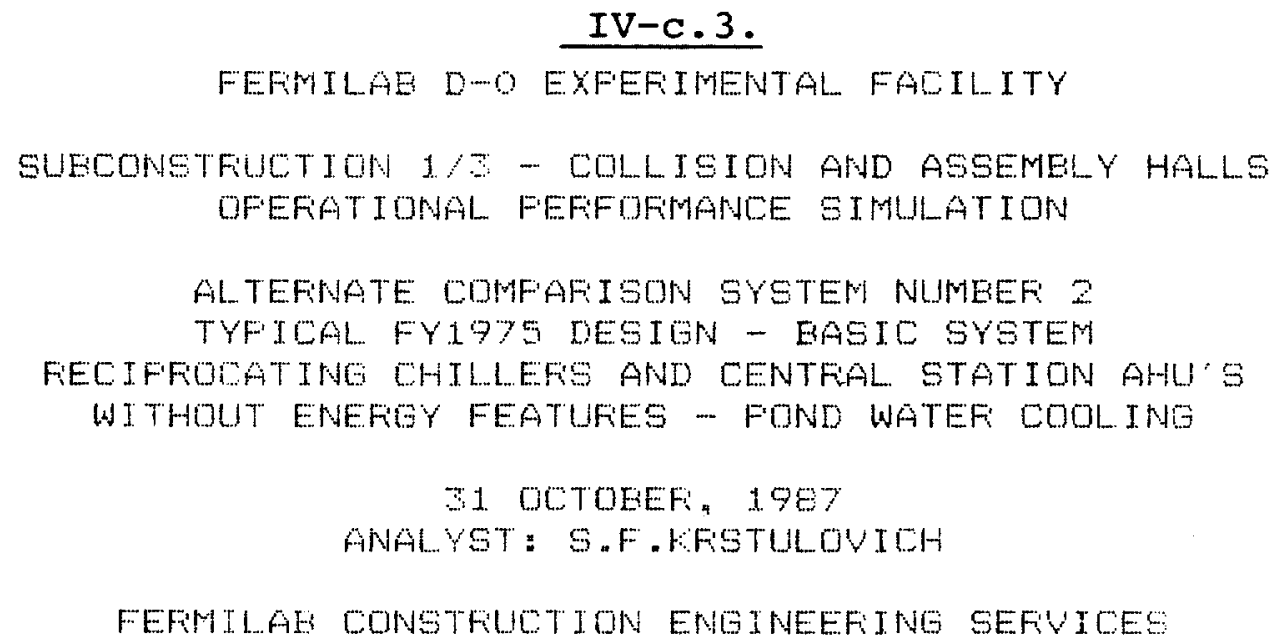


Job Nane : D-O SUECON 1/2 FY1975 SYS

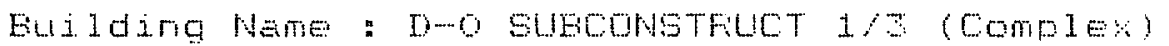

Site : AUFOFA, IL FEFMT, JEer Defined

Date: $11-25-87$

6117962.0

Scope of Anajyeis : Cooling arid Heating Systems

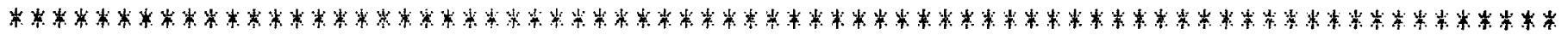

A. AIF HANDLING SYSTEM

1. FEFIMETEF TEFIINAL TYFE " CONSTADT VOLUME

Supply fan type " Fathard inclined or airfoil

Supply fan total static pressure $=4.00$ in. wg.

Fieturn fan type : Forward curved

Fieturn fan total static pressure = 2.00 in. wa.

Design supply air $=42000 \mathrm{cfm}$ e $67 \mathrm{~F}$, Vent. air

Are cooling termina]= used for heating? YY

An economizer is not used.

Is a ventilation peclaim device used $?$ lv

Are ventilation air dampers closed for unoccupied periods $7 \times$

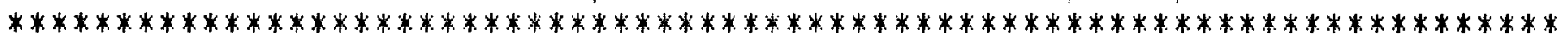

B. HEATINE FLANTS

$\because 1$. FEFIMETEF FLANT : ELETTFICAL FESISTANCE

$I \equiv$ hydromio neatimo used?

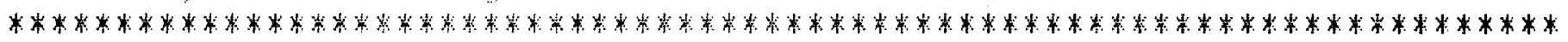




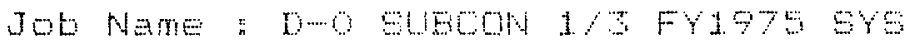

Euilding Name "D-O Eueconstrut $1 / 3$ (Comples)

Fage 2

Site: AUROAA, IL FEFMI, LPE Defined

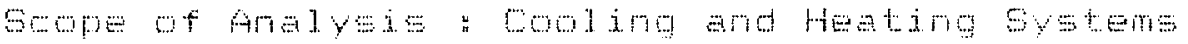

Date : $11-25-67$

60117862.0

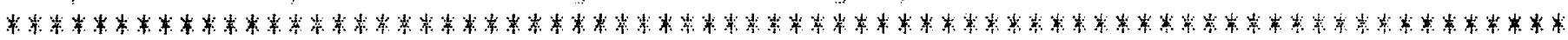

C. COOLING FLANTS

1. FERIMETEF PLANT : RECTFFOCATING HATEF COOLED

Capacity \& $35 \mathrm{~F}$ entering water $=75.0$ tons

rw/Ton d $95 \mathrm{~F}$ entering Hater $=1.00 \mathrm{kw} / \mathrm{ton}$

IE hydronic cooling used ? Yy

Is chilled water reset used ? ly

Is hot gas bypass used $7 \mathrm{~W}$

Is condenser performance altitude adjusted $? \mathrm{~N}$ ?

Is there one compressor per condenser circuit ? Yy

Are compressars cycled ? lv

Heat sinl type : closed circuit cooling tower

Minimum entering water tenperature $=40 \mathrm{~F}$

$I=$ a hydronie ecomomiar used $? \mathrm{~N}$

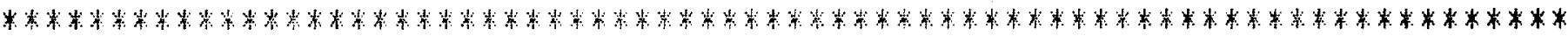

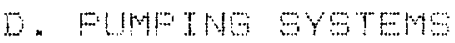

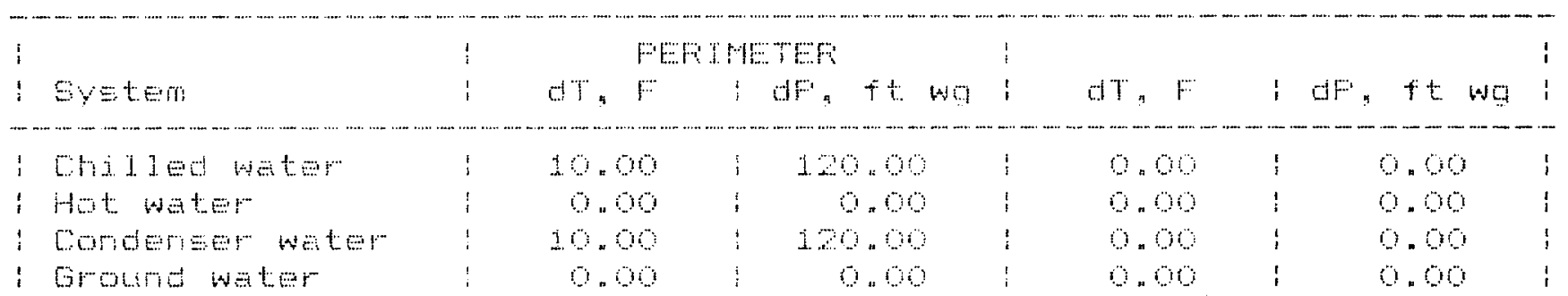

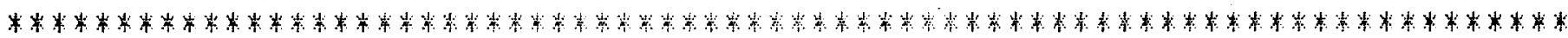

E. NON HYAO SYSTEMS

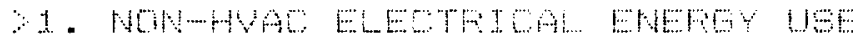

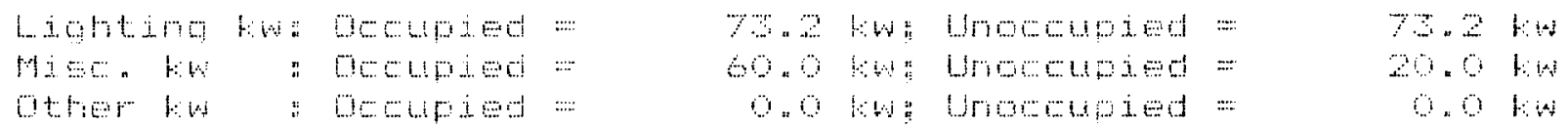

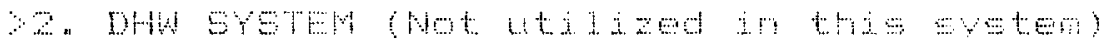

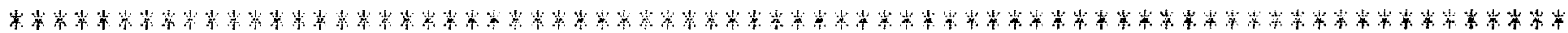
FF. FH:

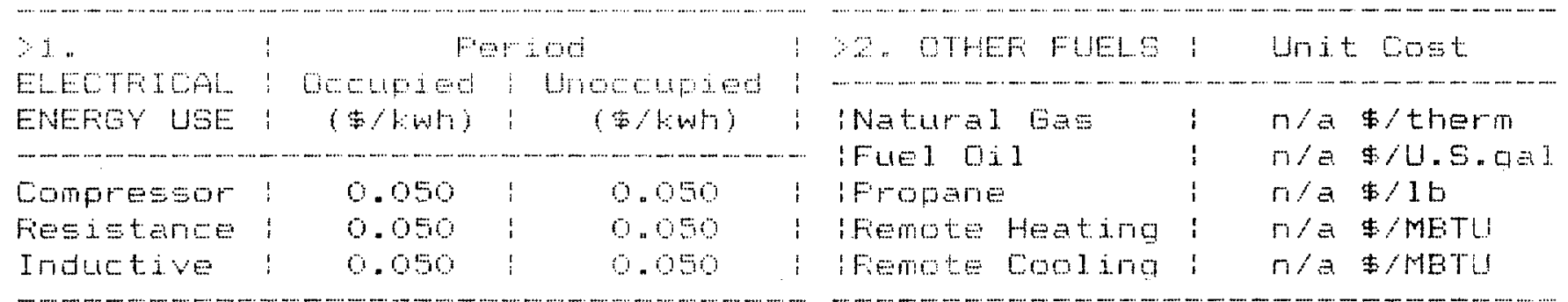


TOTAL BUTHDING ENEFGY SUMMAFY

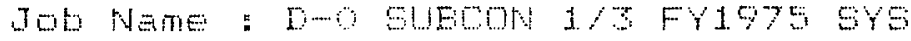

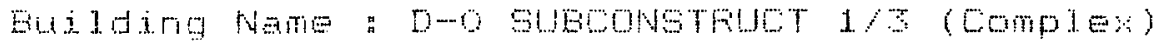

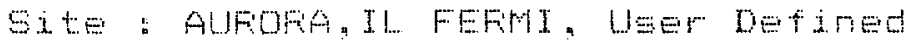

Date: $11-25-87$

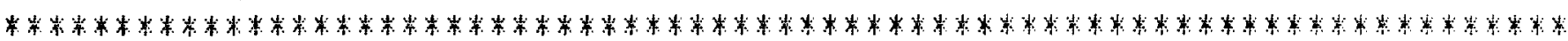

HVAC ENERGY

Comporit:

Electric (Dco)

Electric (unocc)

Natural Gas

Fue 1 Oil 1

Fropane

Fiemote Heating

Finote Cooling

HUAC TotaI
Feriod: Al

zone : Iocl

Serial Number. 60117862.0 Energy or Fum 1
Units

(s)

13711

24572

6

i)

cy

0

i)

.928
DOE FUN

comsumed

274215 kwh/yr

49144 , liwh $/ y^{-}$

o therms/yr

o U.S. ga $1 / y^{2}$

o $1 \mathrm{~b} / \mathrm{yr}$

o MBTU/Yr

O METU/Yr

-

(1000 ETU) (1000 ETU)

$\begin{array}{rr}3180893 & 955895 \\ 5700753 & 1677299 \\ 0 & 0 \\ 0 & 0 \\ 0 & 0 \\ 0 & 0 \\ 0 & 0 \\ -8861626 & 2612189\end{array}$

NON-HVAC ENEFEY

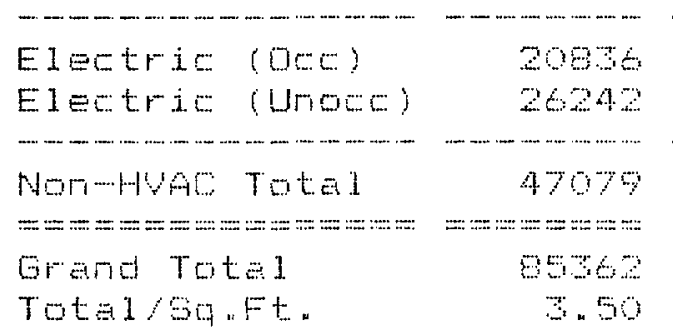

HVAE SUmmery"
HAC Total Cost
$=\quad$ I. $5 \%$ \$ $39 \mathrm{Ft} / \mathrm{yr}$

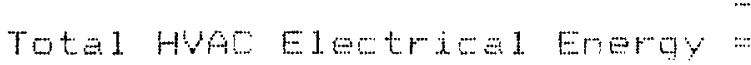
$=44.9 \%$ ot Total Co:

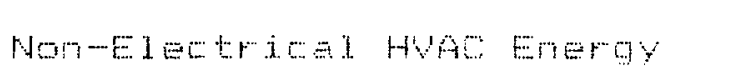

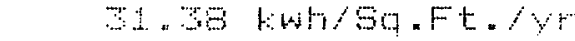
$=4.96 \%$ of Total Electricel Erergy
$=0$ "on " of Total Energy

416700 Hh/

$48 \div 40 \%$

142299

54846 16ithy

$6081 \%$

1791501
109220
$1980590 \%$
011.64

$52+599$

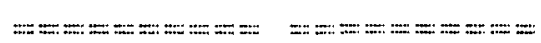

582678

2.80

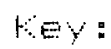
1 tont
$=11600$ ETU FUP a 212 DTU FIF

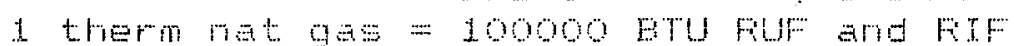
I. U.

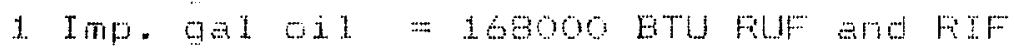
$11 \mathrm{~b}$ propane $=21680$ ETL FUW and FIF
FUUF
FIIF
= Fesolrce utilization factor (Source value)
MEH
= Fesoure impact factor (Foint of use value)
METL
$=1000$ ETU/hr
$=$ Mi.l.j.in ETL 


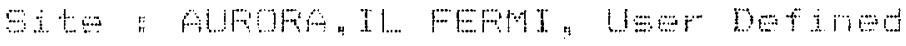

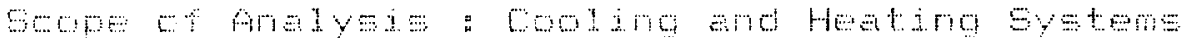

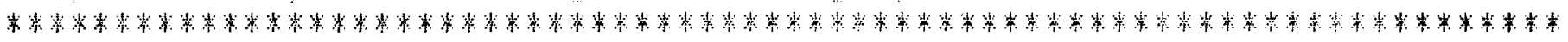

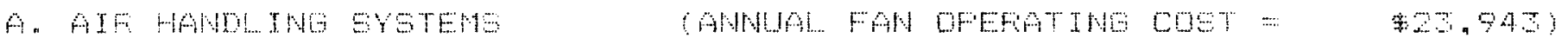

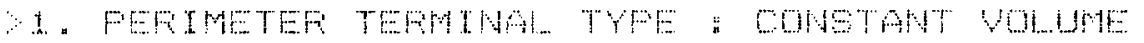

Supply fen type : Buctwerd inelined or airfoil

Supply fen totel state pressure = 4.00 in. wo.

Feturn fan type: Formard curved

Feturm fan totel etatic preseure $=2.00$ in. wa.

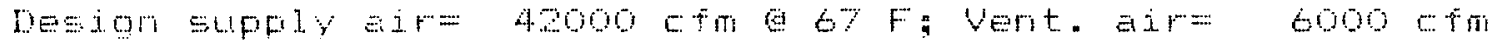

Are cooling teminals used for heating ? Yे

An economizer is not lised.

Is a ventilation reclain device used? w

Are ventilation a dr dampers closed for unoccupied periods ? Na

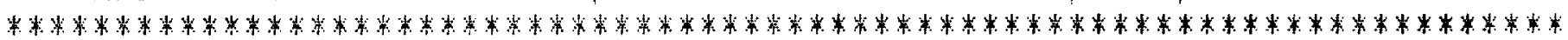

D. HEATING FLANTS IANNUAL HEATING FLANT OFEFATINE COET =

$\$ 5.014$ )

$\checkmark 1$ " PEFIMETEF FIANT : ELETRTCAL FESTSTANCE

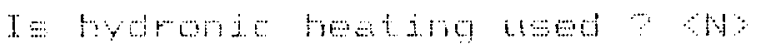

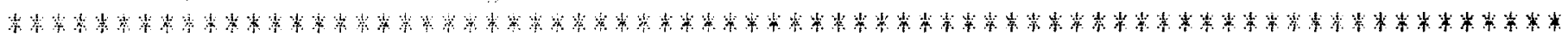




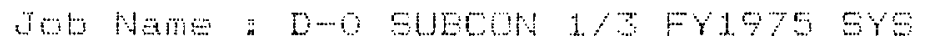

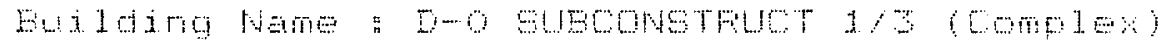

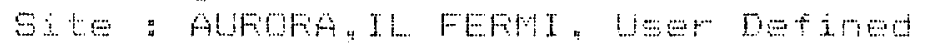

Scome की कnalyo

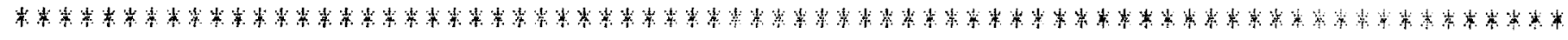

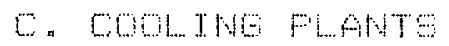

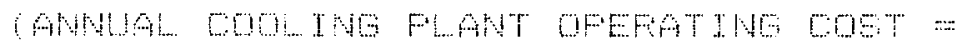

950 : 5

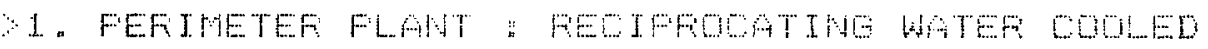

Lapacity a $85 \%$ entering weter $=75.0$ tons

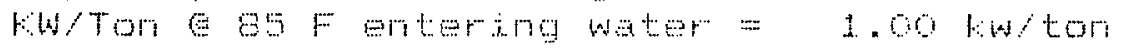

$I=$ hydronde cooling used

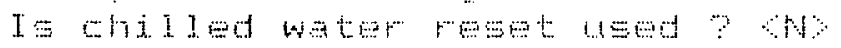

$\mathrm{I}=$ hot gas bypes

Is condenser performanom at itude ad justed ? ads

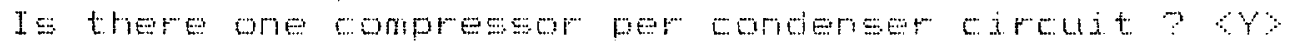
ArE compressors aviled ?

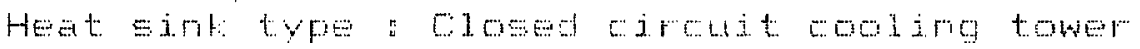

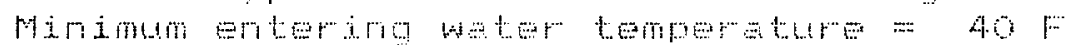

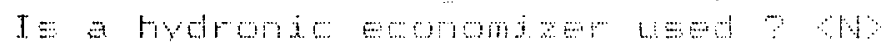

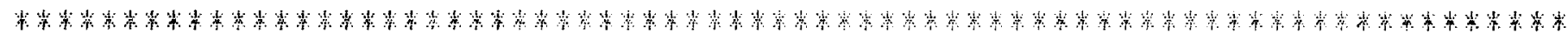

D. FINTIU⿴囗十)

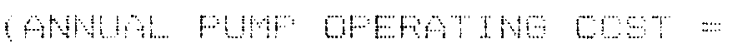

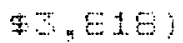

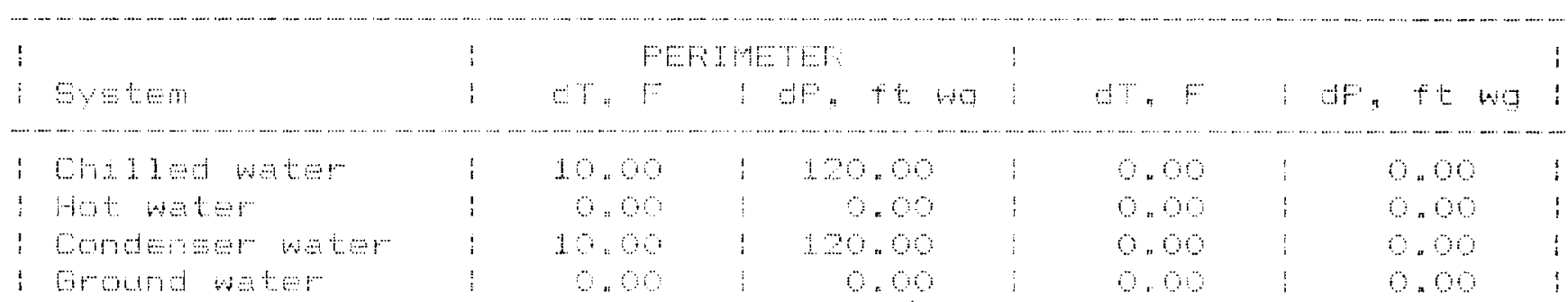

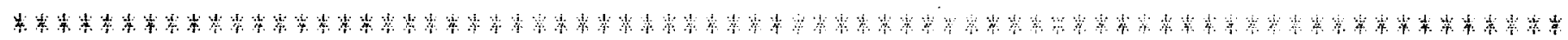

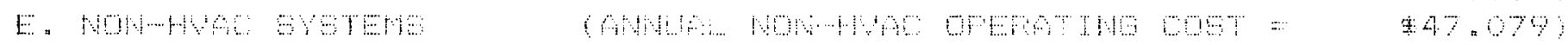

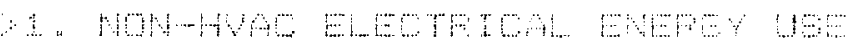

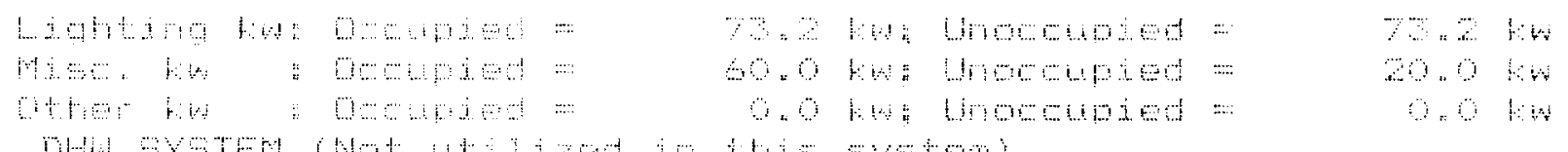

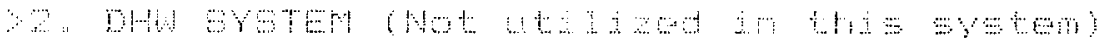

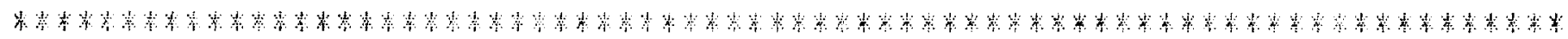

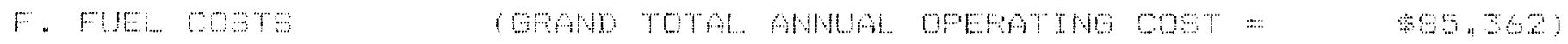

\begin{tabular}{|c|c|c|c|c|c|c|}
\hline 1 & \multicolumn{2}{|c|}{$\cdots+\cdots$} & $i$ & 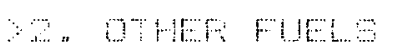 & ! & WH \\
\hline EL]m] & 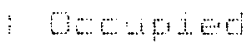 & 41006000 & 1 & ㄱ․ & & 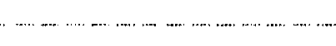 \\
\hline EMEmE & 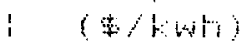 & $(4)(x, b)$ & ; & Matred & 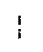 & 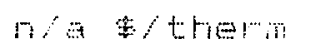 \\
\hline & & 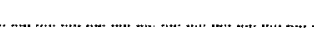 & $\ldots \ldots$ & FLEJ OII & $i$ & क十. \\
\hline Compres $=80$ & 009 & 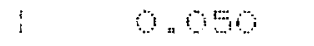 & 1 & mopme & 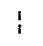 & $5 / \mathrm{b}$ \\
\hline Fes -1 ance & $0.6 \mathrm{~s}$ & a & $i$ & Fenote het ino & i & S/MPTU \\
\hline 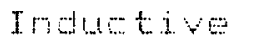 & 0.96 & 9.9 & 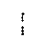 & Mende Godina & $!$ & E/PIETU \\
\hline
\end{tabular}




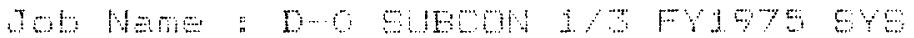

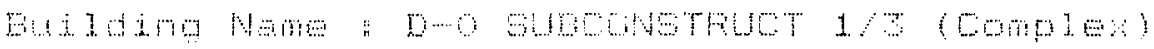

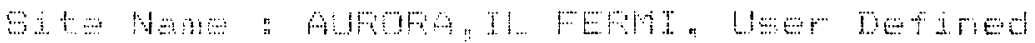

Dote: $; 1+2-6-6$

erian

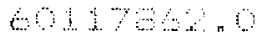

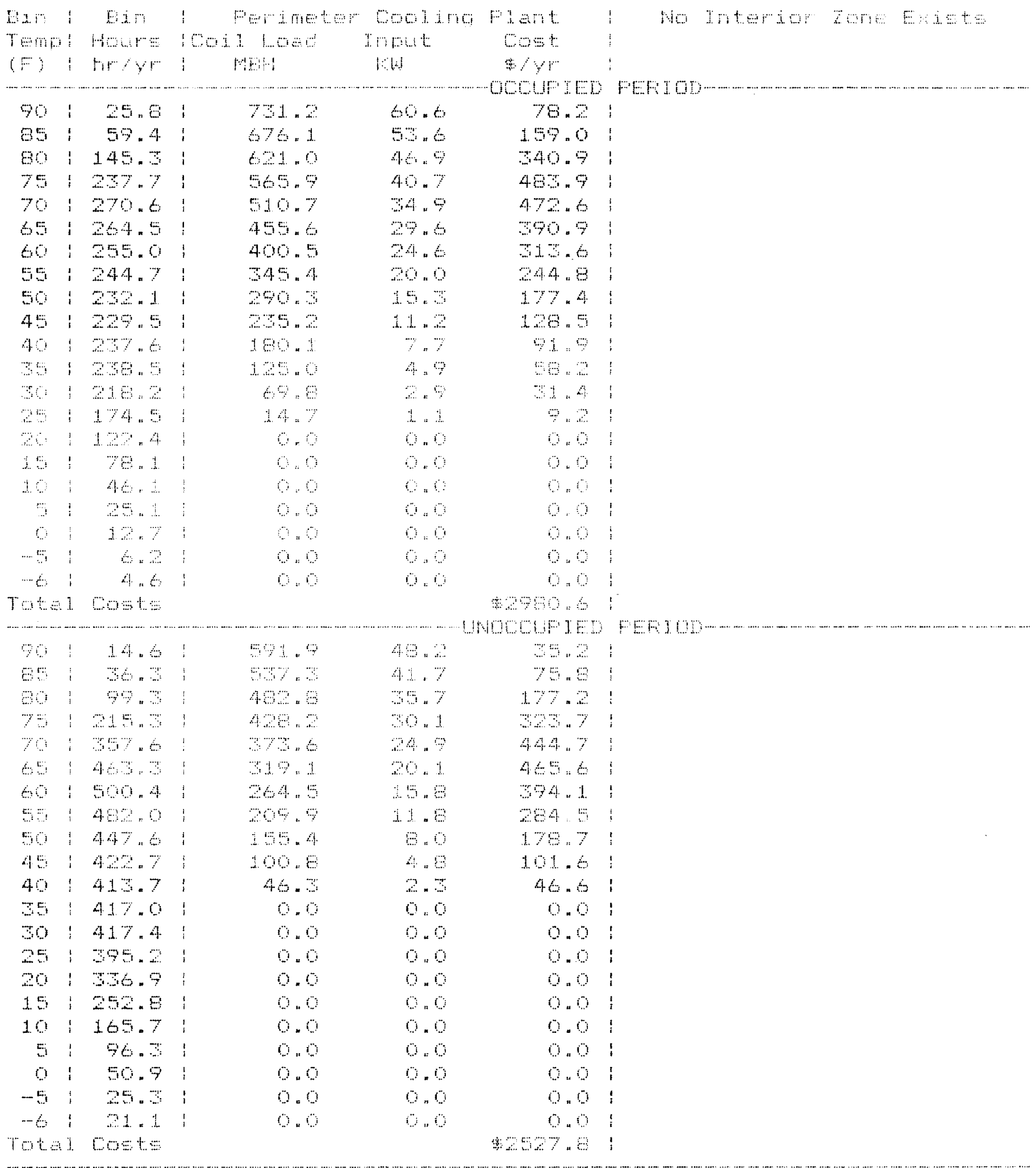


Job name :

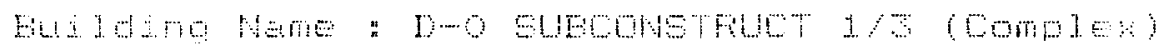

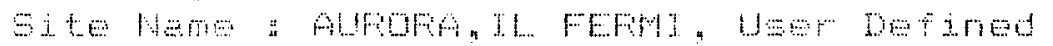

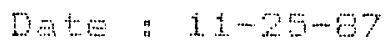

Serial Numer: 6017.720

\begin{tabular}{|c|c|c|c|c|c|c|c|c|c|}
\hline E.I. In & i & 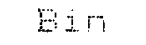 & $i$ & min & $i$ & $m+i$ & Tiaty & ato & : No Alxildary Flant Leed \\
\hline TEm & $:$ & Hotrs & $\vdots$ & Leat & & OLtplt & Intot & E & $i$ \\
\hline$(F)$ & $i$ & $\operatorname{Fr} / \mathrm{Yr}$ & $i$ & MH & $i$ & MEI & U & $4 / \gamma$ & $\vdots$ \\
\hline & & & & & & & 0 & & Wh \\
\hline 90 & 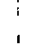 & $=-6$ & $i$ & 0.0 & $i$ & 0 & 0.0 & 0.0 & $i$ \\
\hline$E \ldots$ & 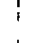 & 57.4 & $i$ & 9.6 & i & 0.0 & 0.0 & 0.0 & i \\
\hline 80 & 1 & 145,3 & $!$ & 9.0 & 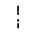 & 0.0 & 0.0 & 0.0 & $i$ \\
\hline 75 & 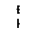 & $2 \% 7$ & $i$ & 0.0 & $i$ & 0.0 & 0.0 & 0.0 & i \\
\hline 70 & $!$ & 270.6 & i & 0.0 & 1 & 0.0 & 0.0 & 0.0 & i \\
\hline 65 & $i$ & 264.5 & $\vdots$ & 0.0 & 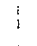 & 0.0 & 0.0 & $0 \%$ & i \\
\hline 60 & i & 255. & $i$ & 9.0 & $\dot{3}$ & 0 & 0.0 & 0.0 & 1 \\
\hline 58 & $i$ & 244.7 & $\vdots$ & 9.0 & $\vdots$ & 0.0 & 0.0 & 0 & 1 \\
\hline 50 & $i$ & $2 a=1$ & $i$ & 0.0 & i & 0.0 & 0.0 & 0.0 & i \\
\hline 45 & $i$ & 229.5 & 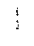 & 0.0 & 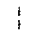 & $\mathrm{o}$ & 0.0 & 0.0 & $!$ \\
\hline 40 & $!$ & 297.6 & $\stackrel{!}{i}$ & 9.9 & 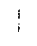 & i. & 60 & 0.0 & $i$ \\
\hline 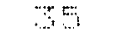 & $\vdots$ & 280,6 & $\vdots$ & $\theta_{n}$ & $!$ & 0.0 & 0. & 0 & $!$ \\
\hline 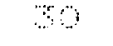 & $\dot{1}$ & 210 & $!$ & in & $\vdots$ & 0.0 & ओ & to & $i$ \\
\hline$\because 5$ & i & 174 & $\vdots$ & $\omega_{n}$ & $!$ & a & $0: 0$ & $\omega, 0$ & $\vdots$ \\
\hline$\because 0$ & $!$ & $22=4$ & $\vdots$ & $40 \cdot 4$ & $!$ & 40, & $1 \perp .9$ & $7 x-4$ & $i$ \\
\hline 15 & : & 7 & $\vdots$ & 9 & i & 95, & 20 ، & 109 & $\vdots$ \\
\hline 10 & $i$ & $A \omega, 1$ & 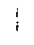 & 190 & : & औ & $\Delta x=1$ & 11.7 & $i$ \\
\hline 5 & $\vdots$ & 26,1 & $\vdots$ & का & 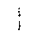 & खीन & का & $75:$ & $i$ \\
\hline 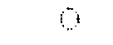 & $!$ & 12.7 & $\vdots$ & $2<0$ & 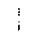 & $2 \operatorname{sol}^{\circ}$ & 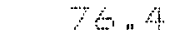 & 40 & $!$ \\
\hline-5 & $:$ & $6 \times$ & 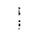 & $\because 16$ & $\vdots$ & 31549 & $92: 6$ & $20: 7$ & $i$ \\
\hline$\cdots$ & : & 4.6 & i & $\because 7: 4$ & $i$ & $x^{\prime}$ & 90 & $2 a$ & $\vdots$ \\
\hline Tota & 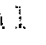 & $m a t=$ & & & & & & 420 & $i$ \\
\hline 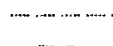 & $\cdots$ & 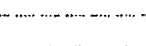 & $\ldots$ & & & (n) & $1010 \%$ & WE FE & 01 \\
\hline 9 & 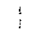 & 14.6 & 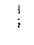 & $\theta_{4}$ & 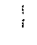 & a & i: & $6: 0$ & $i$ \\
\hline 8 & $i$ & $\because 6$ & 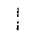 & o & $\vdots$ & औ & a & 0 & $!$ \\
\hline 80 & $\vdots$ & 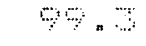 & $\stackrel{1}{\vdots}$ & 1 & $i$ & a & o. & 0. & $\vdots$ \\
\hline 78 & $\dot{i}$ & $\therefore 15,3$ & $i$ & 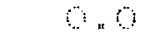 & $\vdots$ & 0 & 0.0 & 0.0 & $\vdots$ \\
\hline 7 & $:$ & 67.6 & 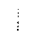 & a & $!$ & 0.0 & ओ & $a \cdot 0$ & 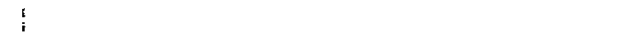 \\
\hline 66 & 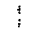 & $46 \%$ & $i$ & i & $\dot{3}$ & 3. & $1: 0$ & 10 & 1 \\
\hline 60 & $i$ & 500 & $i$ & 00 & $!$ & 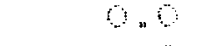 & 0,0 & 0 & $\vdots$ \\
\hline 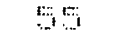 & $i$ & $40=$ & $\vdots$ & 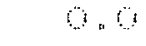 & $\vdots$ & 0 & ओ & $\mathrm{j}$ & 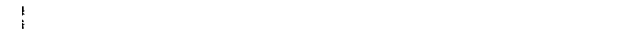 \\
\hline 6 & $!$ & 447.6 & $\vdots$ & ${ }_{a}$ & $\vdots$ & ब, & 0 & बे & $i$ \\
\hline 46 & $i$ & $4 \therefore-7$ & $i$ & 0 & $i$ & ओ & 0.0 & 10 & $\dot{\vdots}$ \\
\hline 40 & $i$ & 413.7 & $i$ & 0.0 & $!$ & 0.0 & 0 & 0.0 & i \\
\hline 75 & $i$ & 4.17 .0 & $i$ & 6 & $i$ & . & $\therefore .4$ & 50.7 & i \\
\hline 30 & i & 417.4 & $i$ & $62 \%$ & ; & $\Leftrightarrow$ & 10.4 & 3044 & $i$ \\
\hline 25 & $i$ & 295.2 & $i$ & 117.4 & i & 117 " 4 & 34,4 & 679.9 & i \\
\hline 20 & 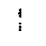 & $x 6.9$ & i & 1720 & $\stackrel{t}{t}$ & 1720 & 90,4 & $848: 9$ & $!$ \\
\hline 15 & i & 2929 & ! & 2060 & i & 26.6 & 6.4 & $58 \% .9$ & 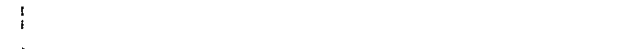 \\
\hline 10 & 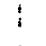 & 165.7 & $i$ & $2 \theta 1,1$ & $!$ & $261-1$ & 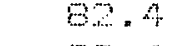 & $68 \%$ 4 & $i$ \\
\hline 5 & i & 96.3 & i & 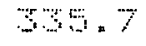 & i & 967 & 98.4 & $47 \square \times 6$ & $i$ \\
\hline 0 & $i$ & 50,7 & $i$ & 390,2 & $i$ & $30 \%$ & 114. & 201.9 & $!$ \\
\hline-5 & ; & 20,3 & $i$ & 44.8 & $i$ & $4 A 4,6$ & 10,6 & $16 A=9$ & $!$ \\
\hline-6 & 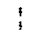 & 21.1 & $i$ & 456 & $i$ & 215.7 & 10 & 140.9 & $!$ \\
\hline Tota & & $6 m s+m$ & & & & & & 54619.6 & $\vdots$ \\
\hline
\end{tabular}




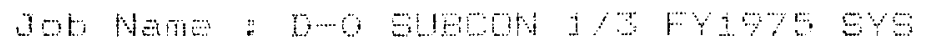

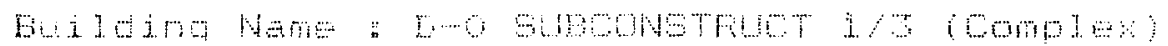

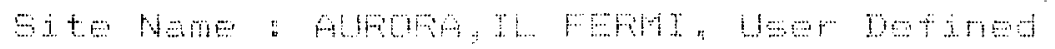

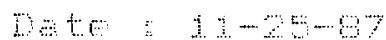

Be, $6+1+2,0$

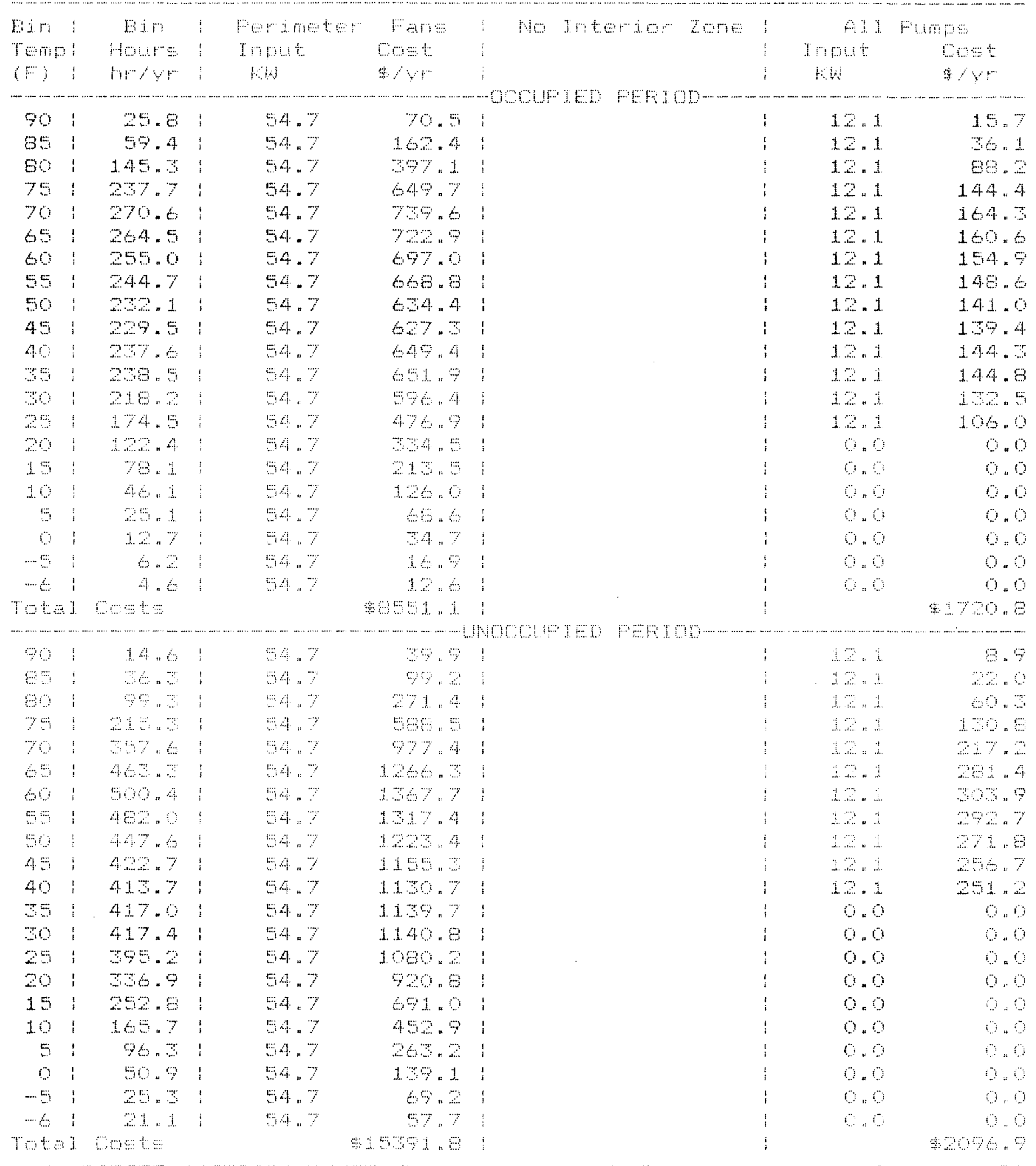




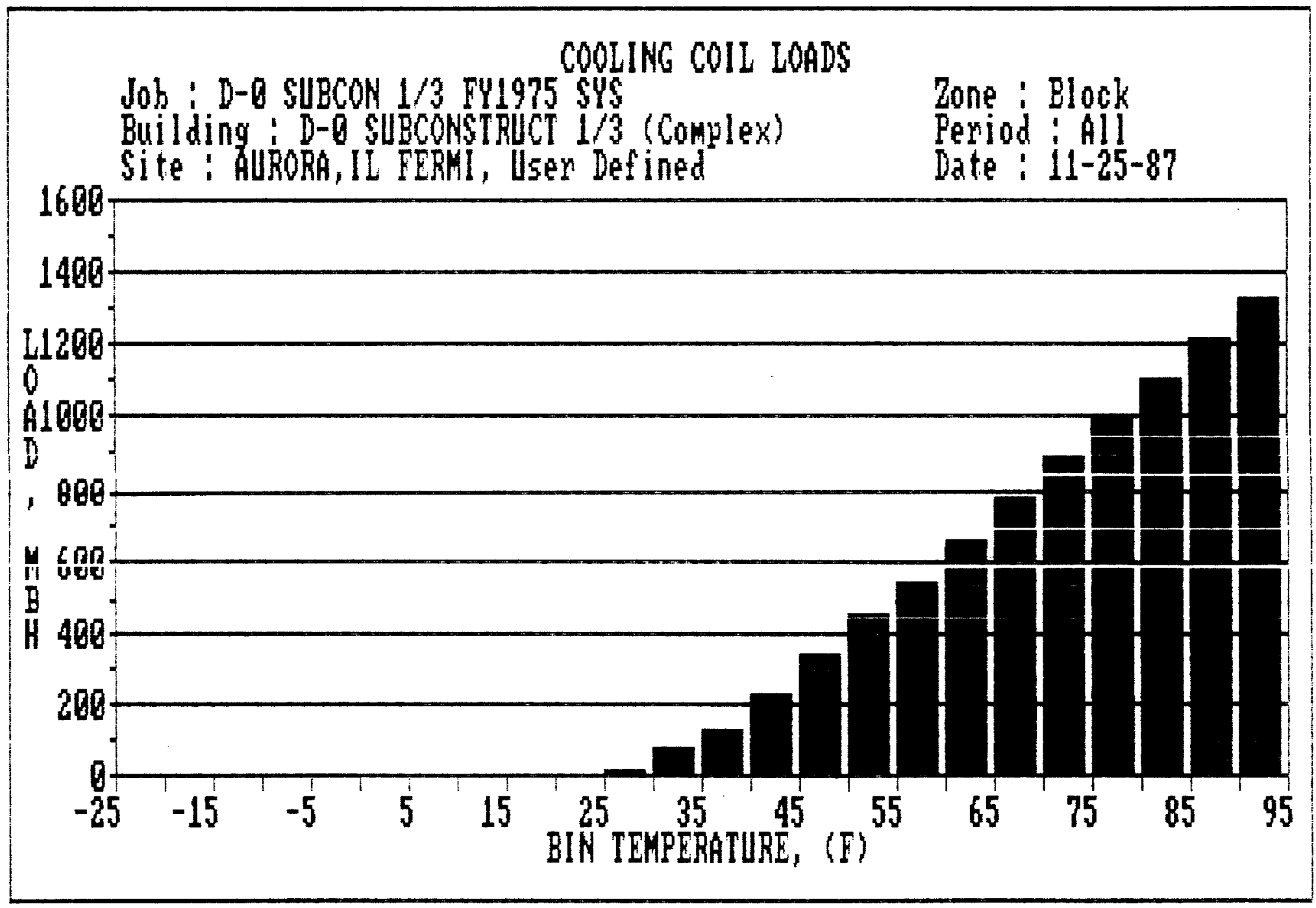




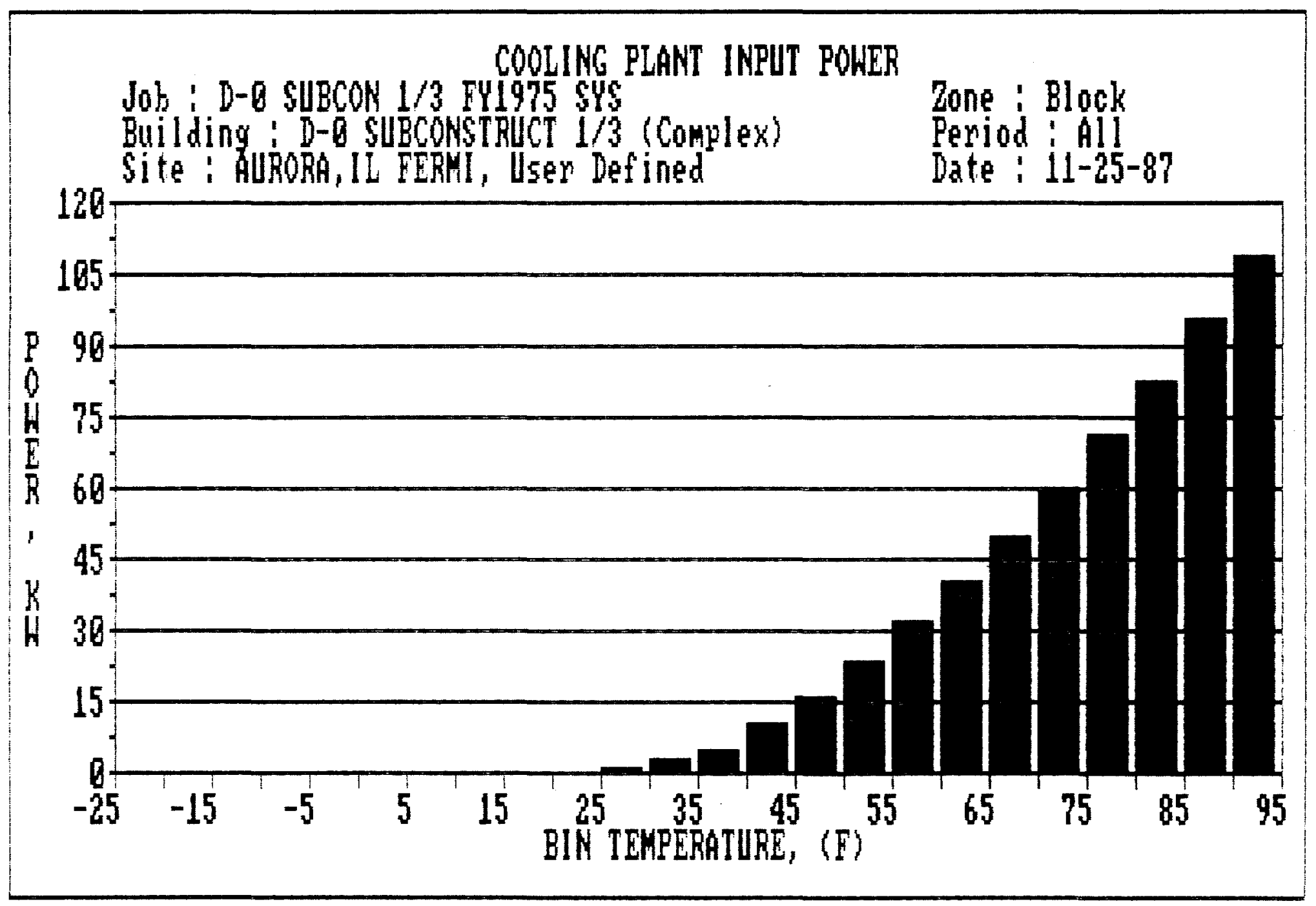


COOLING PLANT OPERATING COSTS

Jot : D-6 SUBCOH 1/3 FY1975 SUS

Building D-0 SlBCOHSIRUCT $1 / 3$ (Complex)

Zone : Elock

Period All

1260
Site: AURORA, IL FERH, User Defined
Site

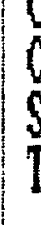

c1658

0

$\$ 900$

750

$\$ 600$

(1)

$$
\begin{aligned}
& y \\
& 8 \\
& B \\
& B
\end{aligned}
$$
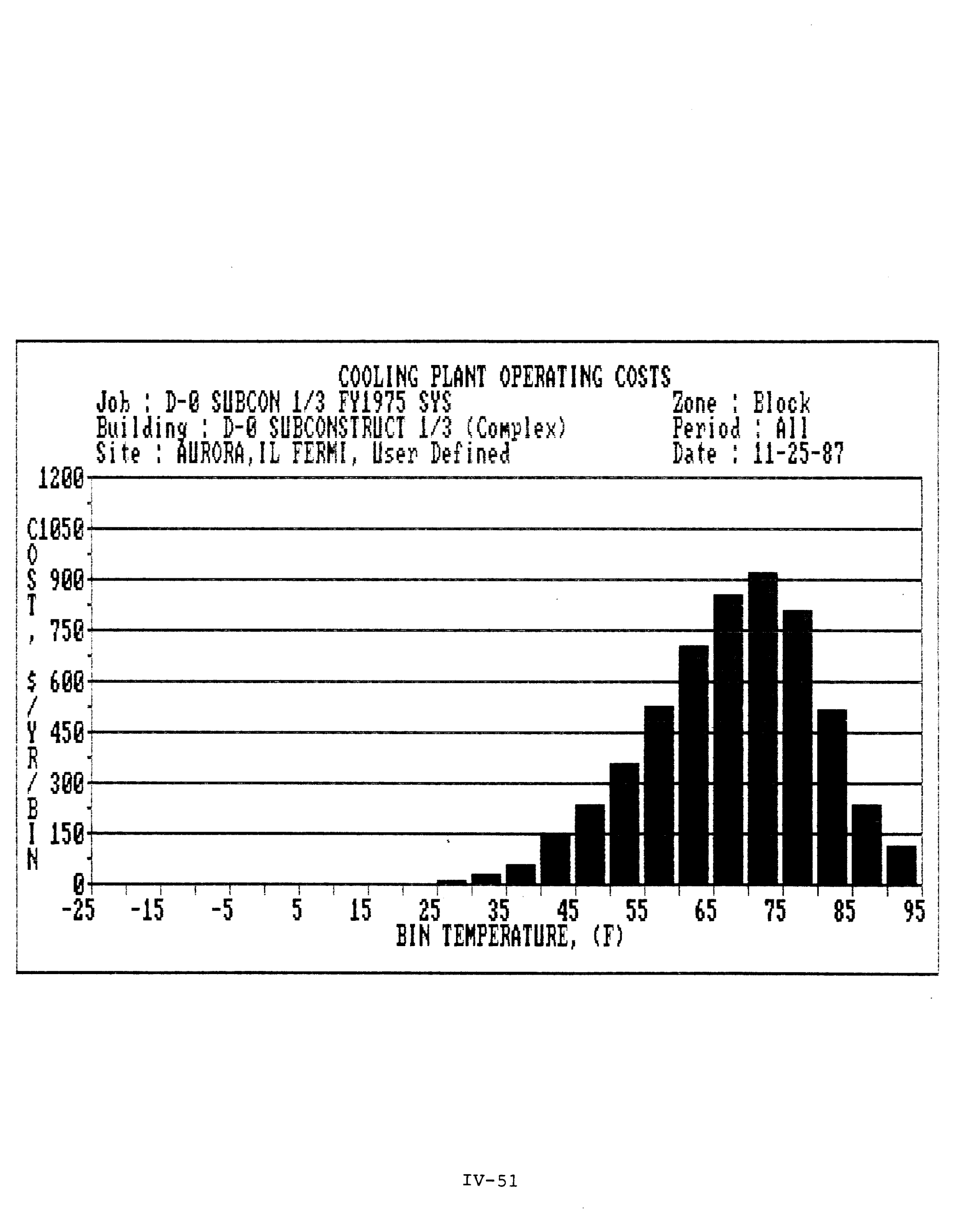


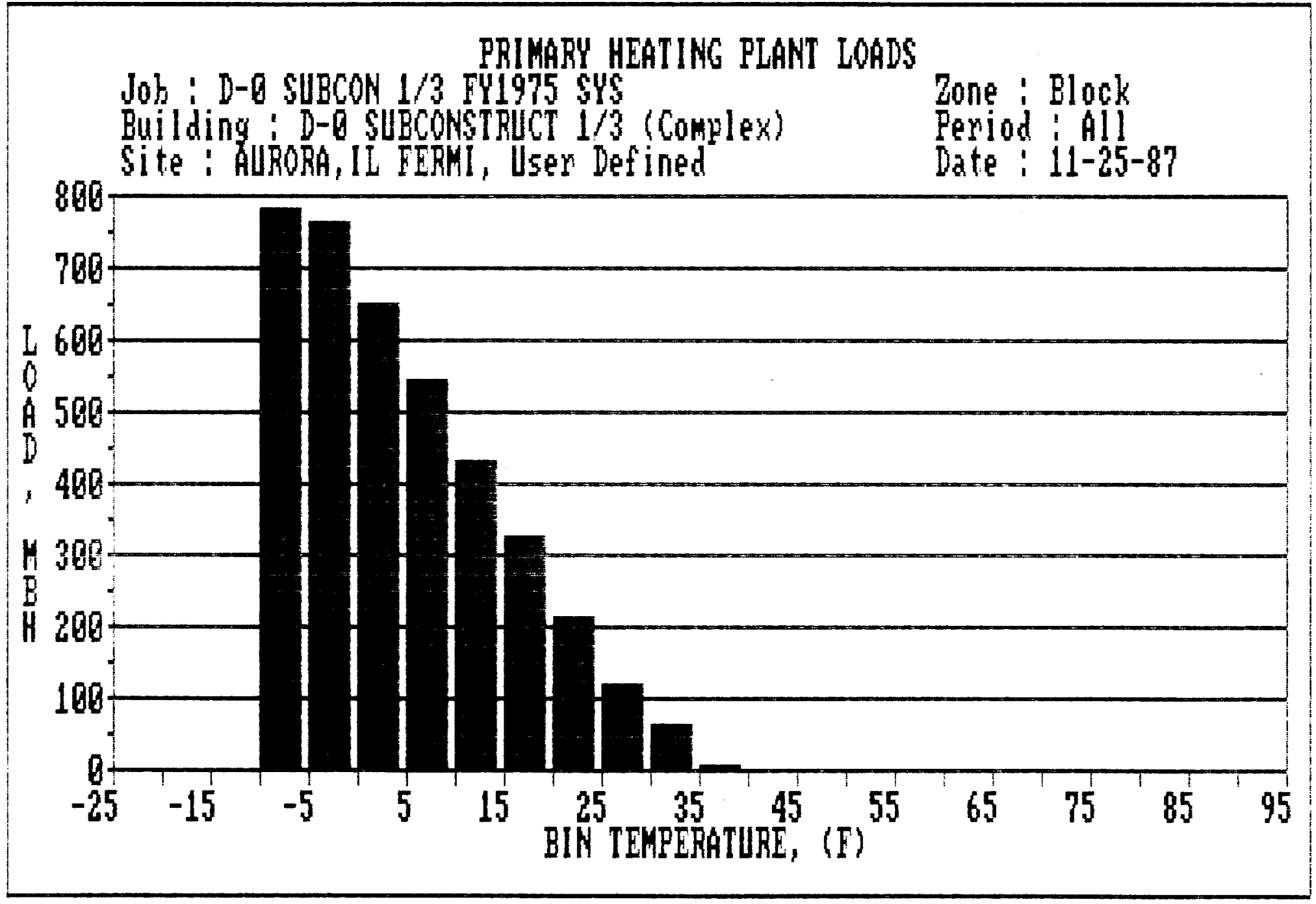




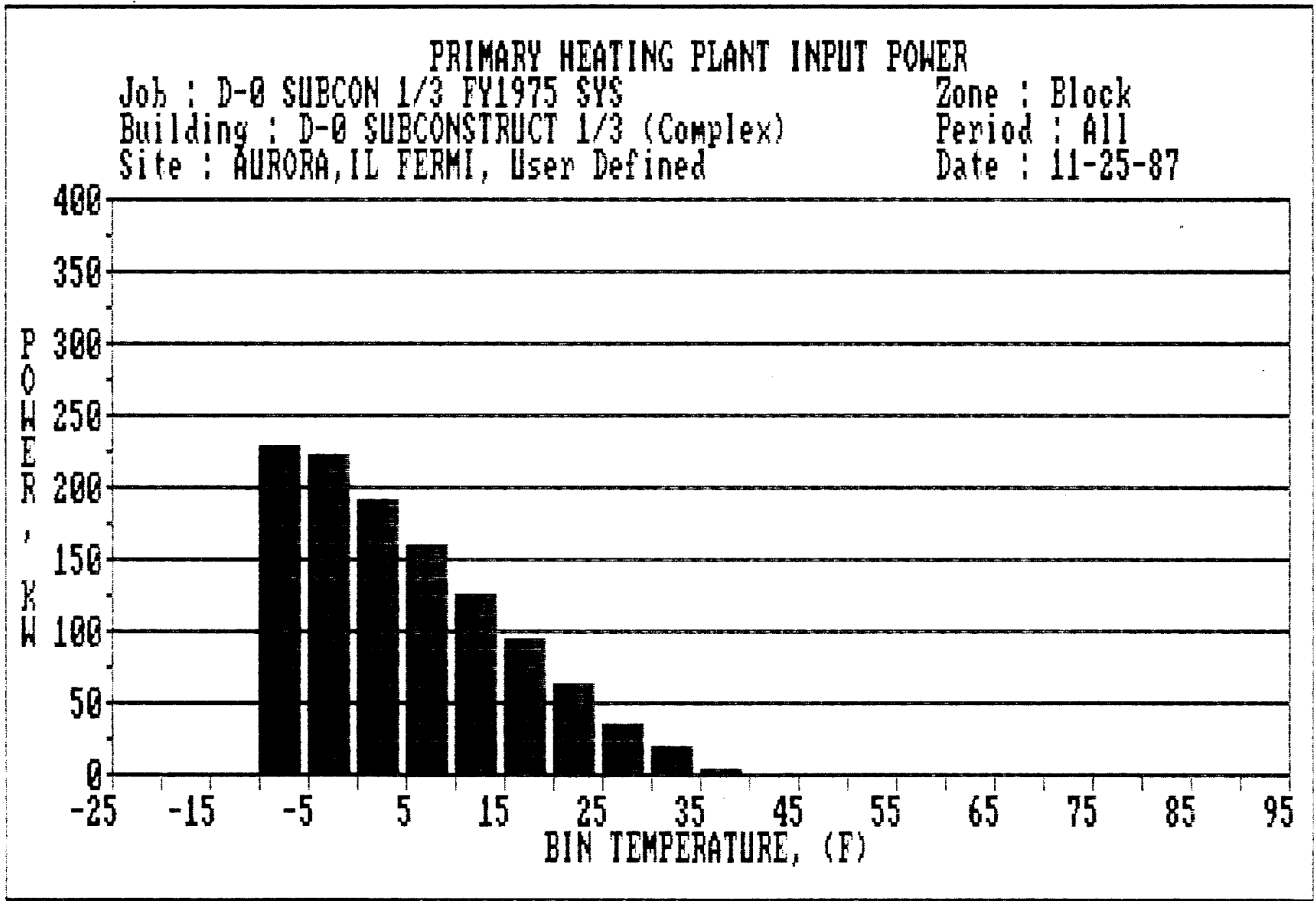




\section{PRIMARY HEATING PLANT OPERATING COSTS}

Joh : D-0 SUBCON $1 / 3$ FY1975 SYS

Building: D-6 sUBCONSTRUCT $1 / 3$ (Complex)
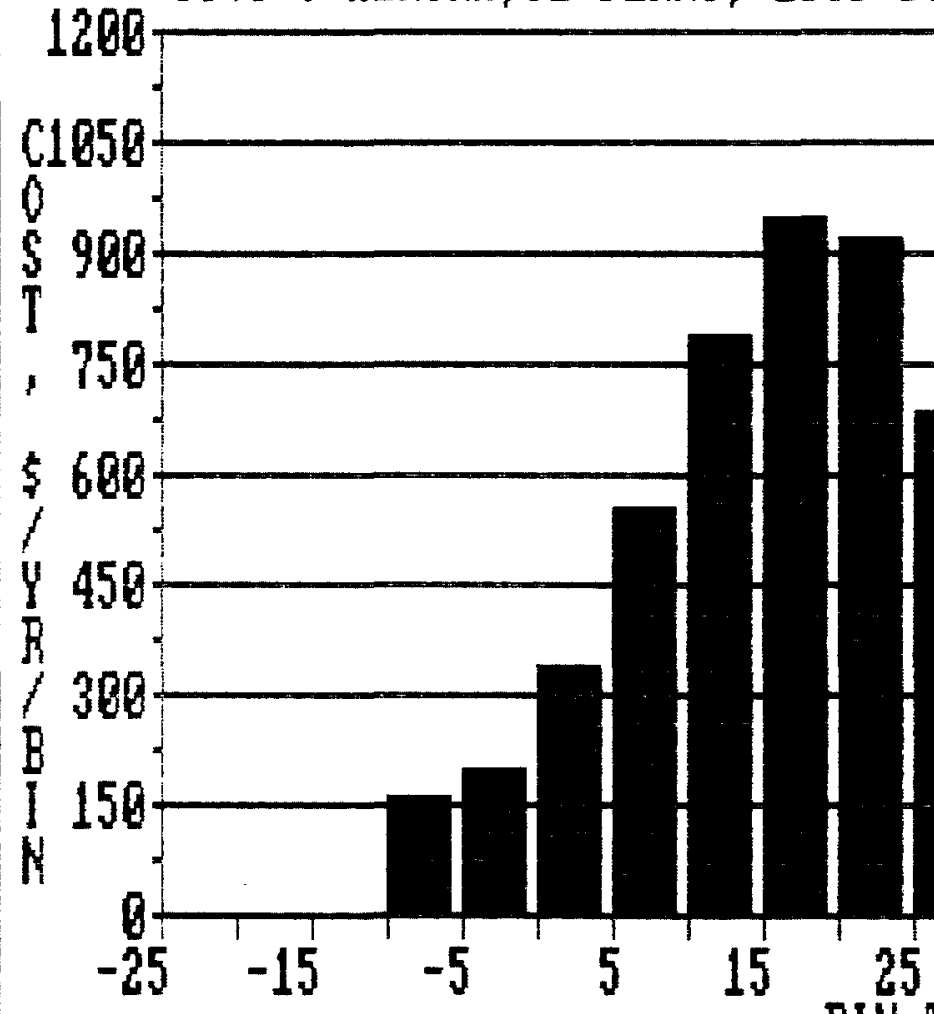

Zone : Block

Period : All

\section{Site: AURORA, IL FERMI, Usen Def ined \\ Date: 11-25-87}




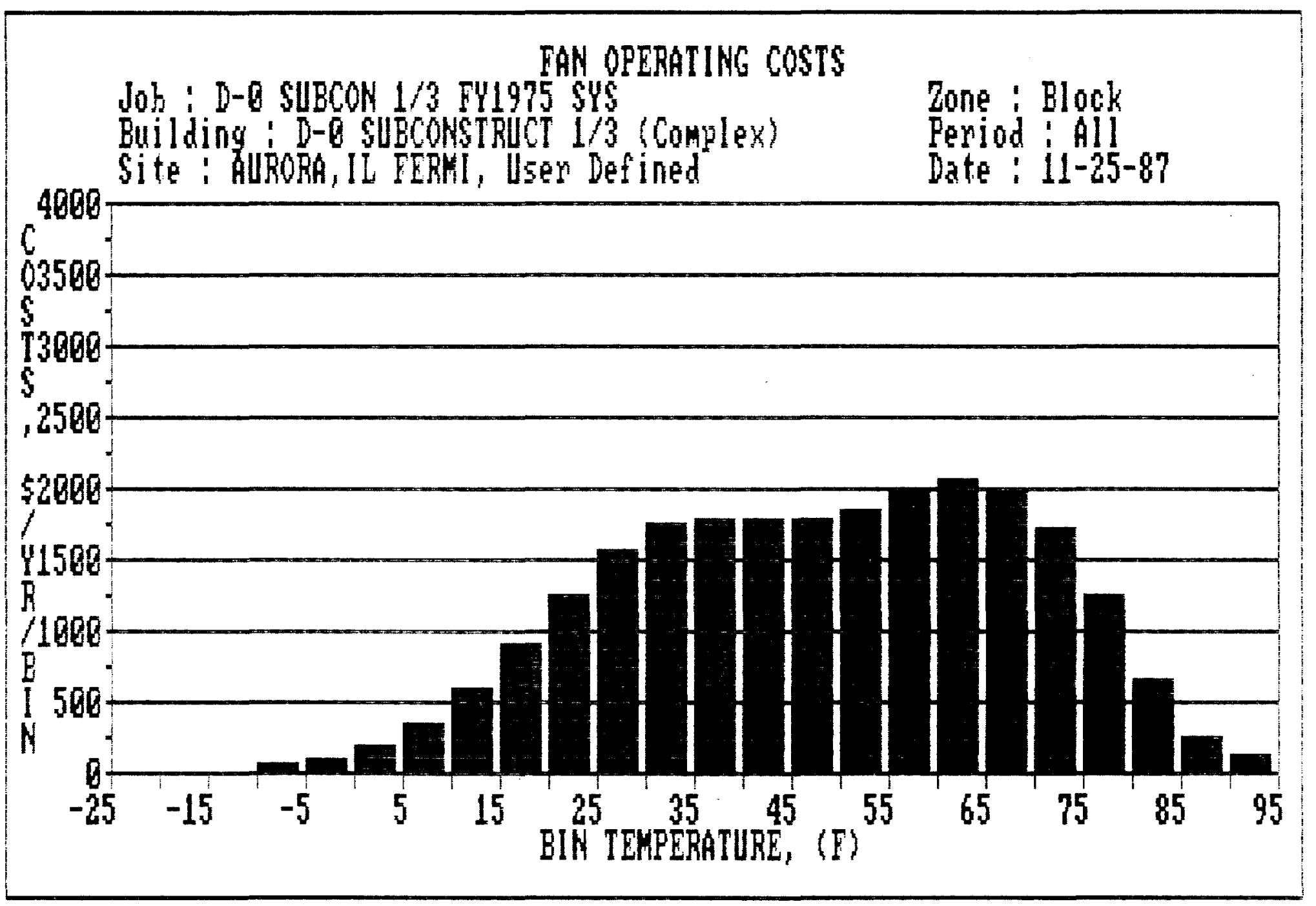


V.

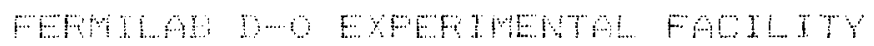

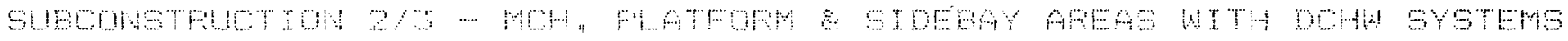
पढा

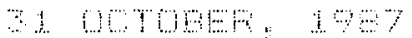

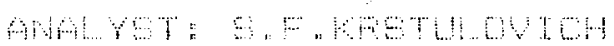

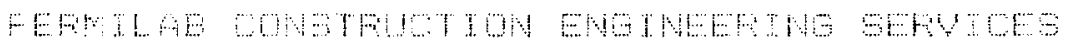


$\mathrm{v}-\mathrm{a}$.

FEFMILAE D-O EXFEFTMENTAL FACILITY

SUECONSTRUCTION $2 / 3-$ MCH, FLATFOFM \& STDEEAY AREAS WITH DCHW SYSTEMS BUILDING SECTOF FHYSICAL FAFAMETEFS AND DESCFIFTION

\author{
31 OCTOEEF, 1987 \\ ANALYST: S.F.FFSTULOVICH \\ FEFMILAE CONSTFUCTION ENGTNEEFING SEFUICES
}

$\mathrm{V}-1$ 
Fuilding Name: D-0 SUECONGTFUCT $2 / 3$

1. FOOM CONDITIONS AWD FLOOF AREAS

Thermostat settings: Cooling $=78 \mathrm{~F}$ : Heating $=68 \mathrm{~F}$; Setbact $=0 \mathrm{~F}$ Warm-up factor $=0 \%$ Foom design reletive humidity $=50 \%$

Floor Areas: Ferimeter $=16,500$ sqft; Interior $=1,300 \mathrm{Sqft}$ Cooling provided during unoccupied period.

\begin{tabular}{|c|c|c|c|c|}
\hline Exposure & $\begin{array}{l}\text { Area } \\
(S q f t)\end{array}$ & $\begin{array}{c}\text { U-Factor } \\
\text { ETU/(hr-sqft-F) }\end{array}$ & $\begin{array}{l}\text { Glass Area } \\
(\text { Sqft) }\end{array}$ & $\begin{array}{l}\text { Glass U-Facto } \\
\text { ETU/(hr-sqft-F }\end{array}$ \\
\hline North Wall & 5,500 & 0.150 & 0 & 1.130 \\
\hline Northeast Wall & 0 & 0.150 & 0 & 1.130 \\
\hline East Wall & 600 & 0.150 & 0 & 1.130 \\
\hline Southeast Wall & 0 & 0.150 & 0 & 1.130 \\
\hline South Wall & 0 & 0.150 & 0 & 1.130 \\
\hline Southwest Wall & 0 & 0.150 & 0 & 1.150 \\
\hline West wall & 600 & 0.150 & 0 & 1.1 .50 \\
\hline Northwest Wail & 0 & 0.150 & 0 & 1.1 .30 \\
\hline Ferimeter Foof & 4,400 & 0.150 & 0 & 0.550 \\
\hline Interior Foof & 0 & 0.150 & 0 & 0.550 \\
\hline
\end{tabular}

WALLS: Color = Dart: Wt. = Medium: FOOF: Color = Medium: Wt. = Medium

EUILDING WT. = MEdIUT: VEFTICAL GLASS : Single Glazed

Shade Factors: Glass = $=1.000$ : Stylights $=0.000$

Internal shades are not used. ; A ceiling plenum is not used.

- L IGHTE:

Ferimeter: $:$.000 Watts/Saft $=$

Interior : Snoo watts/sott =

Diversity Factors : Decupied $=100 \%$ :

Ferimeter Lightse : Ballest recesed

Interior Lights : Ballest , exposed
49.500 Watte Total

3.900 Wats Total

Unoctupied $=9 \%$

4. MISCELLANEOUS ELEETFICAL LOADS:

Ferimeter : Decupied = 12.727 Hatte/5aft $=$ Unoceupied $=4.242$ watts/Sqft $=$

Iriterior : Decupied $=\% 46.154$ Watt $\$ 15 q f t=$ Unoccupied $=\% 115.385$ watts/saft =
210.000 watts Total
70,000 Watts Total
450.000 Watts Total
150.000 Watts Total

5. FEQFLE LOADS

Total Dccupancy $=\quad 593$ sqft/person $=$

Activity Leve1: 2. Office or retail

Sensible $=245.0$ ETU/hr/person; Latent $=205.0$ BTU/hr/person

Diversity Factors: Dccupied $=70 \%$ : Unoccupied $=20 \%$

6. MISCELLANEOUS INTEFNAL LOADS:
Sensible : Occupied =
O ETU/hr: Unoctupied =
O ETU/RF
Latent : Occupied $=$
O ETU/hr: Unoccupied =
O ETU/RF

7 " WALLS ADJACENT TO NON CONDTTIONED REGTONS:

Wall Areas : Ferimeter $=\quad 7.900$ Saft: Interior $=$ o saft

Wal 1 U-Factor $=0.320$ ETU/ (hm-gft-F)

Adjacent region heating temperature $=60 \mathrm{~F}$

Adjacent region cooling temperature $=60 \mathrm{~F}$

8. TNF TL...TFATION DATA:

Air Flow Fates: Occupied =

50 Efin: Unoteupied =

offm 
$\mathrm{V}-\mathrm{b}$

FEFHTLAE D-O EXPEFIMENTAL FALILTTY

SUECONSTFLCTTON 273 - MUH. FLATFOAM 2 STDEBAY AFEAS WITH DCHW SYSTEMS BUILOTNG LOAD CALEULATTONS

31 OCTOEEF, 1987

ANALYST: S.F.FFSTLLOVICH

FEFNILAE CONSTRUOTION ENGTNEEFING SEFUICES 
Euideno Name : D-0 SUECONGTFUCT 23 (Complex)

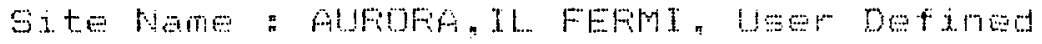

Dets: $: 11-16-87$ 60117862.0

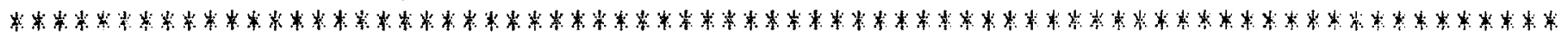

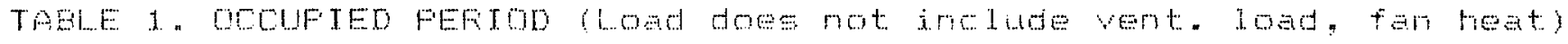

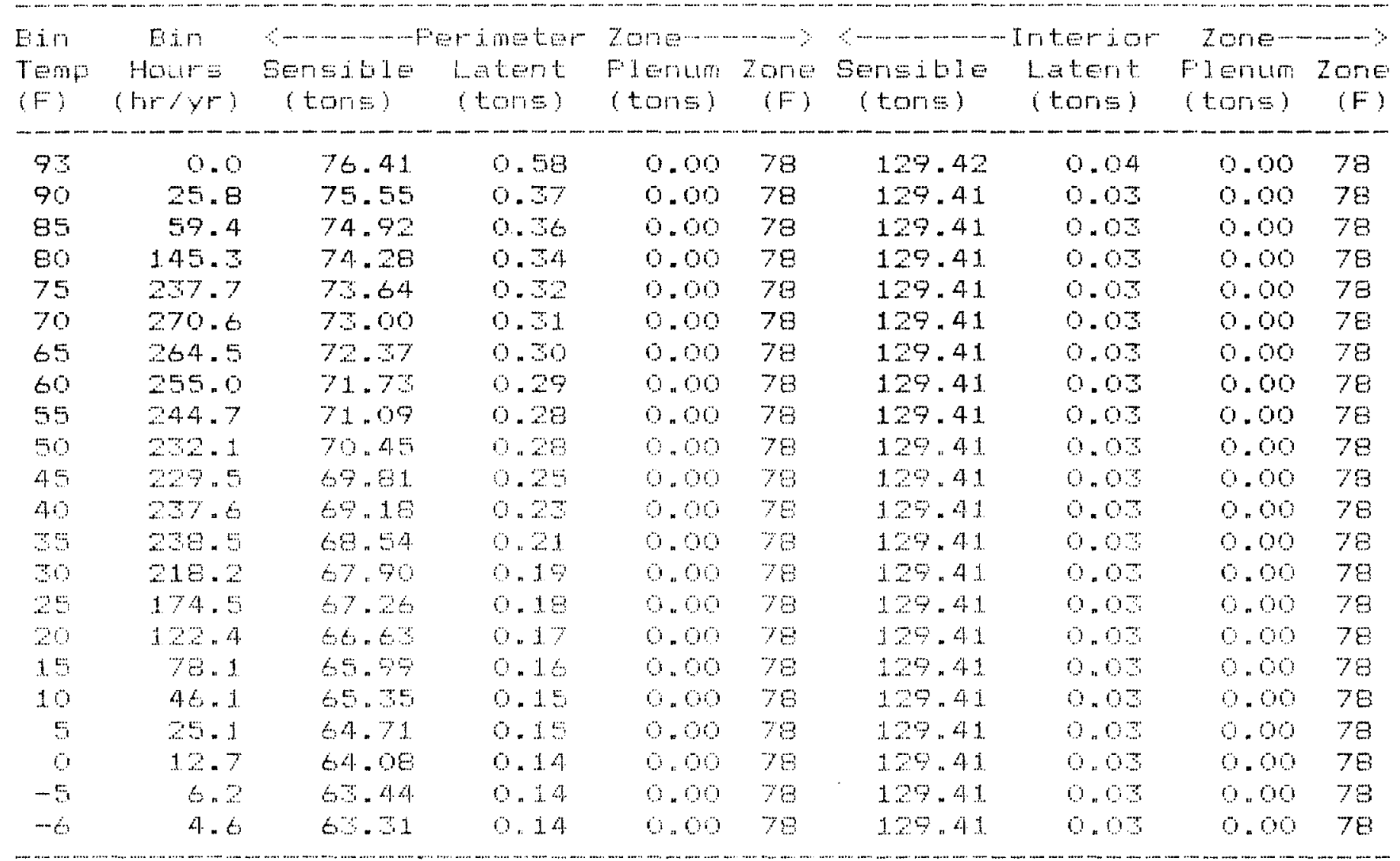

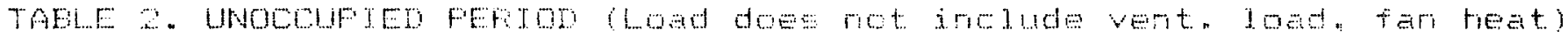

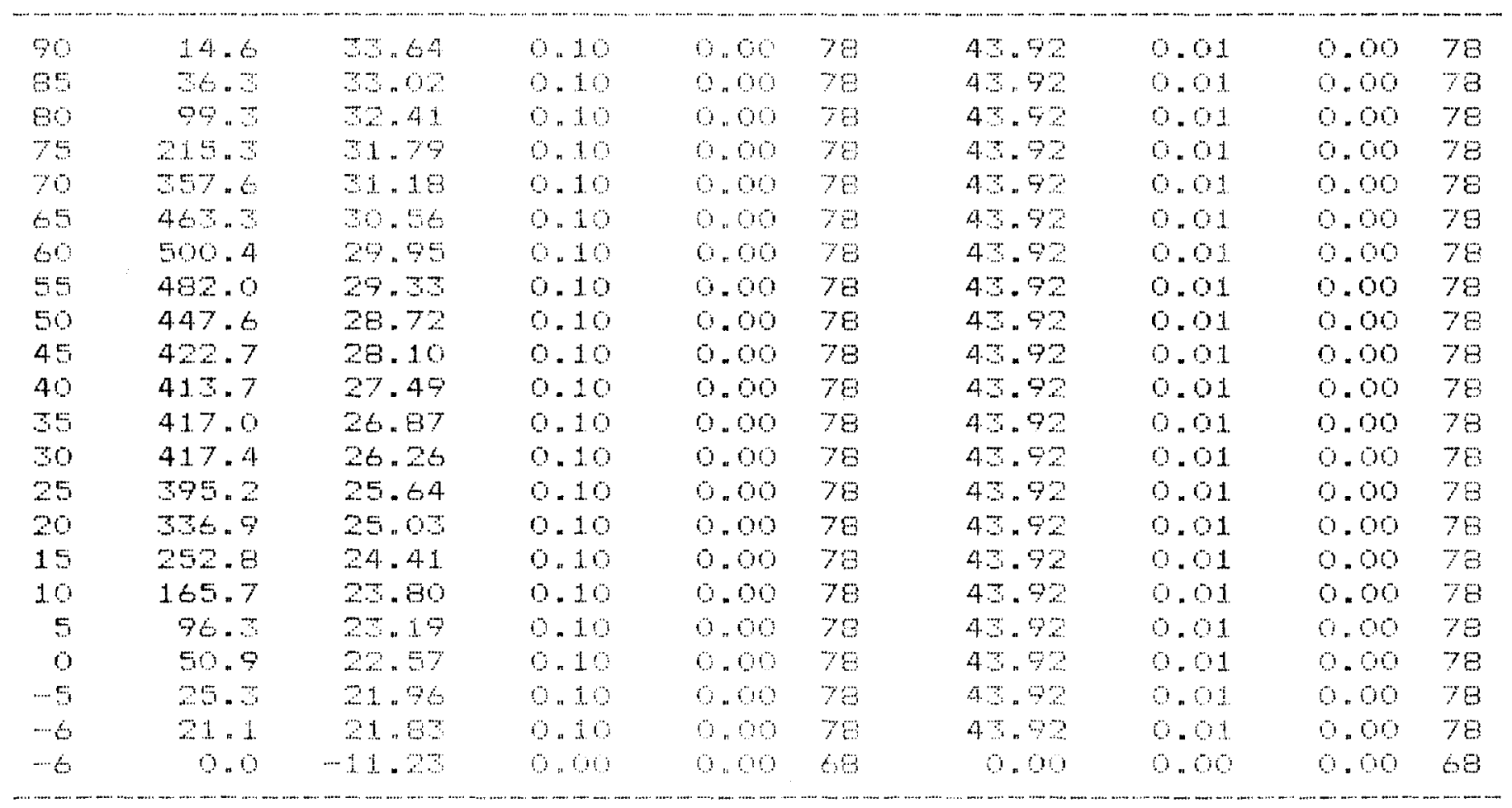


Building Name : D-O SUBCONSTFUTT 2/3 (Comples)

Site Name : AUFORA, IL FEFMI, JeEr Defined

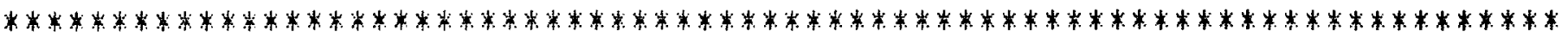

TAELEE 2. SQLAF GAINS EY EXFOSUFE

\begin{tabular}{|c|c|c|c|c|c|c|c|c|c|c|}
\hline \multicolumn{3}{|c|}{$\begin{array}{l}\text { Ein } \\
\text { Temp }\end{array}$} & \multicolumn{6}{|c|}{$\begin{array}{c}\text { Solar Gains by Exposure } \\
\text { (ETu/ (hr-sqft)) }\end{array}$} & \multirow{2}{*}{$\begin{array}{l}\text { Ferim } \\
\text { Horiz }\end{array}$} & \multirow{2}{*}{$\begin{array}{l}\text { Inter } \\
\text { Horiz }\end{array}$} \\
\hline & North & NE & East & $S E$ & South & $S W$ & West & $\mathrm{NW}$ & & \\
\hline 93 & $1 I_{0}$ & 50.4 & 47.4 & 5.6 & 50.4 & 74.4 & 56.9 & 27.5 & 0.0 & 0.0 \\
\hline 90 & 9.2 & 21.5 & 5.6 & 38.1 & 35.9 & 52.9 & 40.4 & 19.5 & 0.0 & 0.0 \\
\hline 85 & 8.8 & 20.4 & 3.0 & 30.3 & 34.4 & 50.5 & 58.6 & 18.5 & 0.0 & 0.0 \\
\hline 80 & 8.5 & 19.5 & 50.3 & 34.6 & 32.9 & 48.1 & 20.7 & 17.5 & 0.0 & 0.0 \\
\hline 75 & 7.8 & 18.2 & 28.7 & 2.9 & 31.4 & 45.8 & .34 .7 & 16.5 & 0.0 & 0.0 \\
\hline 70 & 7.4 & 17.1 & 27.1 & 31.2 & 29.9 & 4.5 & 50 & 15.5 & 0.0 & 0.0 \\
\hline 65 & 6.9 & 16.0 & 25.4 & 29.4 & 28.4 & 41.0 & \pm 1.2 & 14.5 & 0.0 & 0.0 \\
\hline 60 & 6.5 & 1.4 .9 & 23.8 & 27.7 & 27.0 & 38.6 & 29.5 & 13.5 & 0.0 & 0.0 \\
\hline 55 & 6.0 & 13.8 & 22.3 & 26.0 & 25.5 & 36.3 & 27.5 & 12.6 & 0.0 & 0.0 \\
\hline 50 & 5.5 & 12.6 & 20.5 & 24.2 & 24.0 & 33.9 & 25.6 & 11.6 & 0.0 & 0.0 \\
\hline 45 & 5.1 & 11.5 & 18.9 & 22.5 & 22,5 & 51.5 & 20.6 & 10.6 & 0.0 & 0.0 \\
\hline 40 & 4.6 & 10.4 & 17.5 & 20.0 & 21.0 & 29,1 & 21.9 & 9.6 & 0.0 & 0.0 \\
\hline 35 & 4.1 & 9.5 & 15.6 & 19.0 & 19.5 & 26.6 & 20.1 & 8.6 & 0.0 & 0.0 \\
\hline 30 & 5.7 & 8.2 & 14.0 & 17.3 & 10.0 & 24.4 & 10.2 & 7.6 & 0.0 & 0.0 \\
\hline 25 & $\Xi 2$ & 7.1 & 12,4 & 15.6 & 16.5 & 22.0 & $1 \in .4$ & 6.6 & 0.0 & 0.0 \\
\hline 20 & 2.8 & 6.0 & 10.7 & 13.9 & 15.0 & 19.6 & 14.5 & 5.6 & 0.0 & 0.0 \\
\hline 15 & 2.3 & 4.7 & 9.1 & 12.1 & 13.5 & 17.7 & 12.7 & 4.7 & 0.0 & 0.0 \\
\hline 10 & 1.8 & $\exists .8$ & 7.5 & 10.4 & 12.0 & 14.9 & 10.8 & $\exists .7$ & 0.0 & 0.0 \\
\hline 5 & 1.4 & 2.7 & 5.8 & 8.7 & 10.6 & 12.5 & 9.0 & 2.7 & 0.0 & 0.0 \\
\hline 0 & 0.9 & 1.6 & 4.2 & 6.9 & 9.1 & 10.1 & 7.1 & 1.7 & 0.0 & 0.0 \\
\hline-5 & 0.4 & 0.5 & 2.6 & 5.2 & 7.6 & 7.6 & 5.5 & 0.7 & 0.0 & 0.0 \\
\hline-6 & 0.8 & 0.2 & 2.3 & 4.9 & 7.3 & 7.3 & 4.9 & 0.5 & 0.0 & 0.0 \\
\hline-6 & 0.0 & 0.0 & 0.0 & 0.0 & 0 & 0.0 & 0.0 & 0.0 & 0.0 & 0.0 \\
\hline
\end{tabular}


ETD SUMMAFYY

Euilding Name: D-o SUECONGTFLCT 2/5 (Complex) Date $11-16-87$ Site Name: AUFIOFiA, IL. FEFHI, UEer Defined

60117862.0

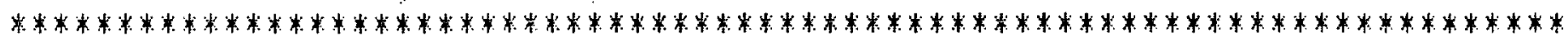
TAEILE 1. DCCUFIED FEFITOD

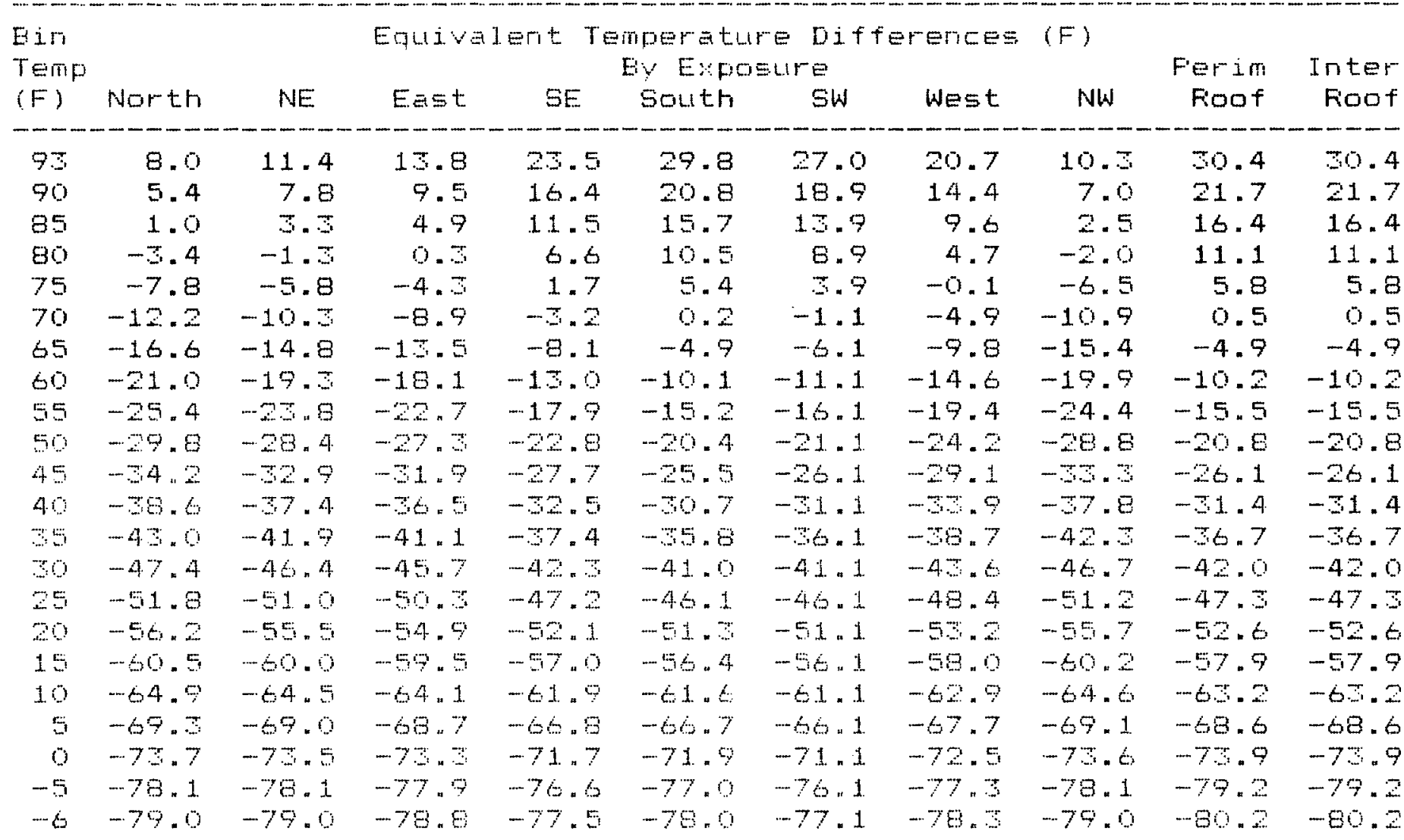

TADLE 2. WHOCCUFIED FEFIOD

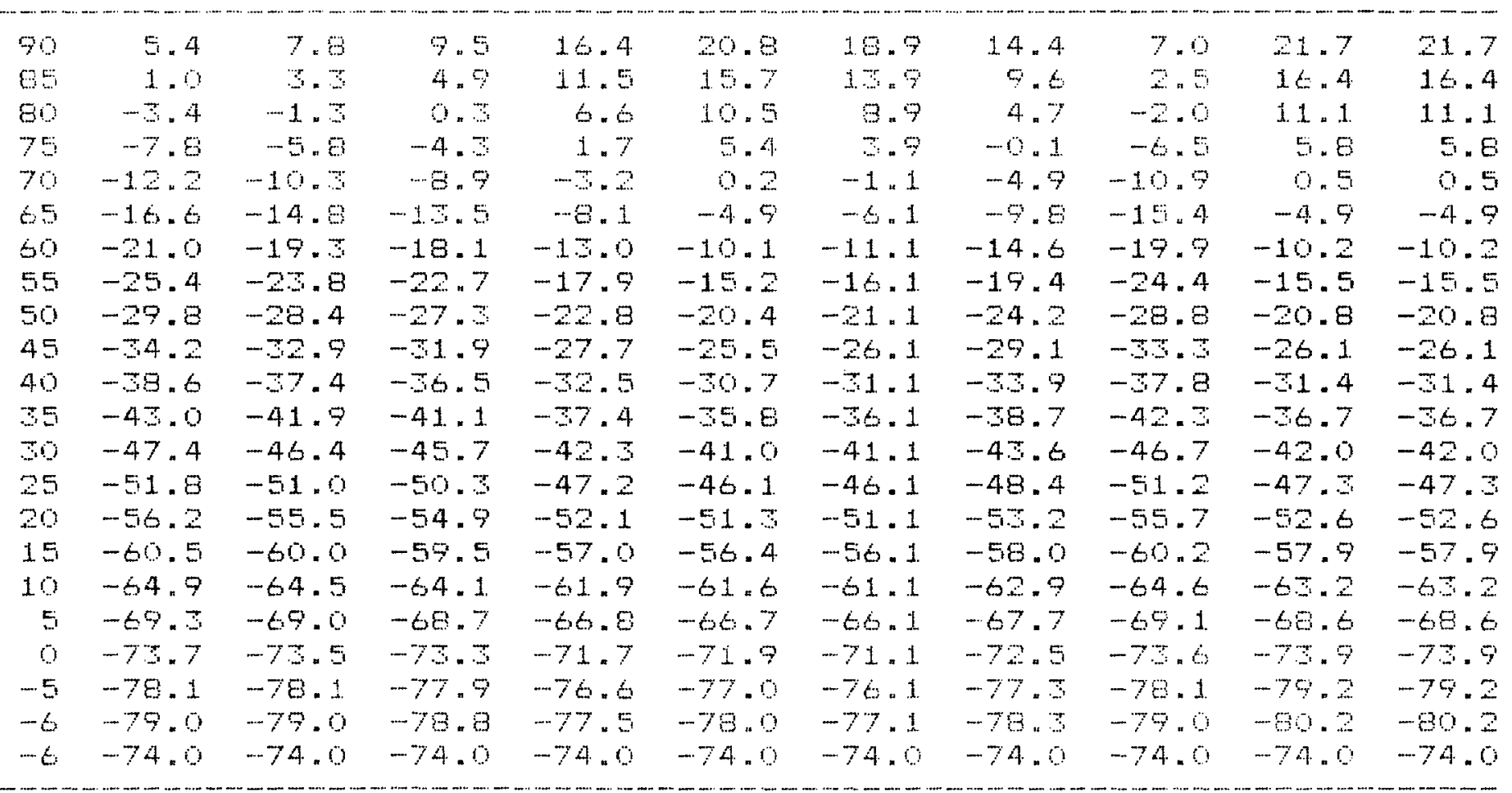


$\mathrm{V}-\mathrm{C}$.

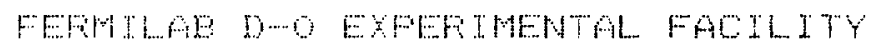

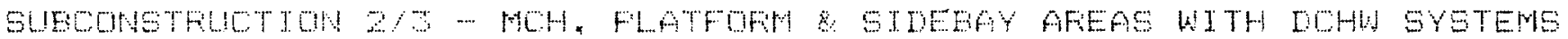

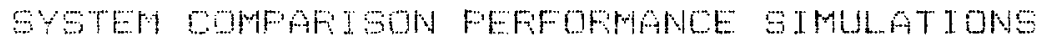

अ

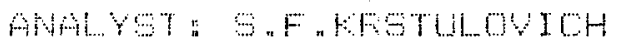

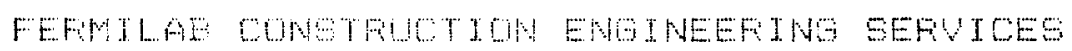

$\mathrm{V}-7$ 


\section{$\mathrm{V}-\mathrm{c} \cdot-1$}

FEFMILE D-O EXFEFIMEMTAL FAOILITY

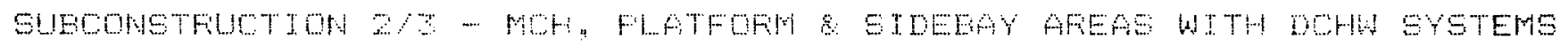
OFEFAT TONAL PEFFOFMANEE SINULATION

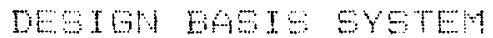

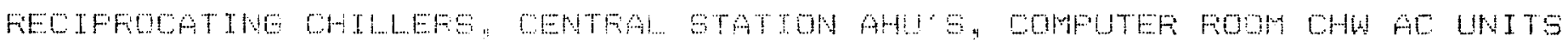

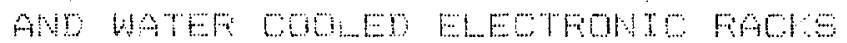
WTH THESE ENEROY FEATUES:

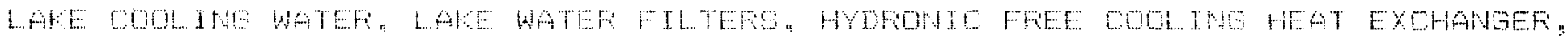

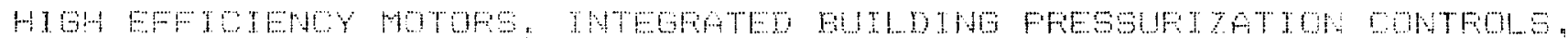

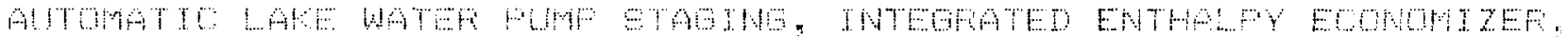

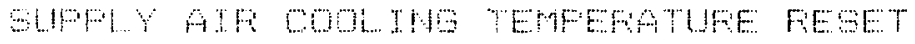

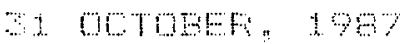

mpl. $6 \%$ "

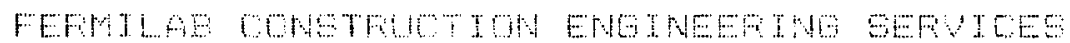


Job tame : D-0 sueCon 2y DESIOH SYS

Building Name : D-0 sueconstruet $2 / 3$ (Complex)

Site : AUFOFA, IL FEFin, UEer Derimed

Scope of Analysis: Cooling and Heating Systeme

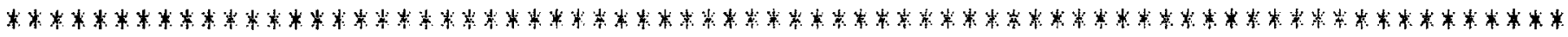

A. AIF HANDL ING BYSTEMS

fre perimeter and interior zone on same supply fan $?$ a

1. PEFIMETEF TEFMINAL TYFE : CONSTANT VOLUME

Supply fan type: Forward curved

Supply fan totel static pressure = 2.00 in. wg.

Feturn fan type: Controlled pitch axial

Feturr fan total static pressure = 1.00 in. wg.

Design supply air $36000 \mathrm{ctm}$ d $5 \mathrm{~F}$ Vent. air= $600 \mathrm{cfm}$

Are cooling terminals used for heating ? Yy

Ecomomizer type : Integrated, enthalpy controlled

Is a ventilation reclaim device used?

Are ventilation air dampers closed for unoccupied periods $?$ s.

2. INTEFIOF TEFMINAL TYFE " CONSTANT VOLUME

Supply fan type: Formerd curved

Supply far totol static pressure = $0.01 \mathrm{in}$. wo.

A return fan is not used.

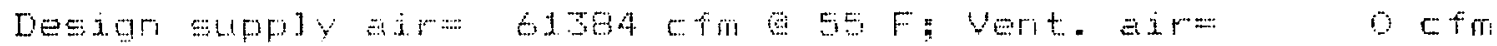

An economizer is not bsed.

Is a ventilation reclain device used?

Are ventilution eir dampers elosed for uroccupied periods $=\mathrm{N}$

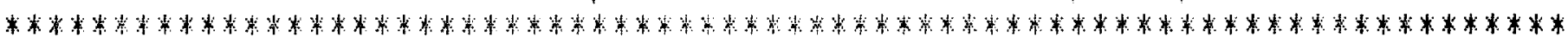

E. HEATTNG FIANTS

Does one primary heating plant eerve perlmeter and interior ? Yy

31 . BLOCH FLANT : ELECTFTOA! FESTSANOF

Is hydronie heating used?

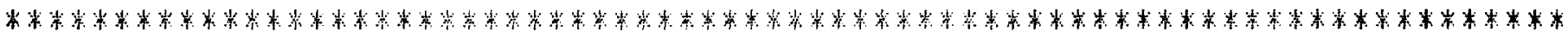


Job Name: D-0 SUBCON 2. DESTGN SYS

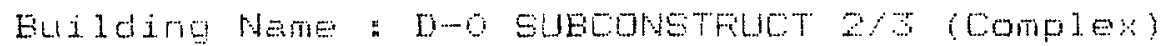

Fage 2

Site : AUFDFA, LL FEFMI, UEEr Defined

Scope of Anelysis : [ooling and Hëtjng Systems

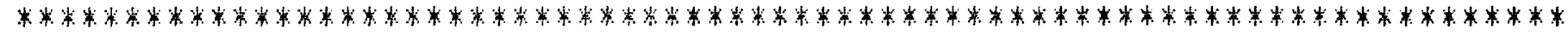

D. COOL ING FLANTE

Does one plant ester perimeter and interiar $\because$ y

1. BLOCF FLANT : FECIFFOCATING WATEF COOLED

Capacity a $85 \mathrm{~F}$ entering water $=230.0$ tons

rw/Ton e $85 \mathrm{~F}$ entering water $=0.85 \mathrm{kw} / \mathrm{ton}$

Is hydronic cooling used ? YY

Is chilled werter reset $115 \mathrm{sed} ? \mathrm{~N}$

Is hot gas bypass used ? W

Is condenser performance altitude adjusted $? \gamma Y$

Is there one compressor per condenser circuit $?$

Are compressors cyeled $?$ Y Y

Heat sint: type a Open cooling tower

Minimun enterimg water temperstue $=32 F$

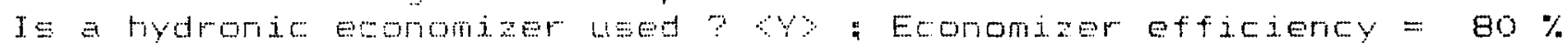

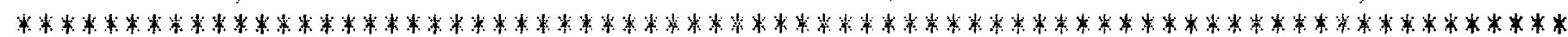

D. FUVIFING SYSTEMS

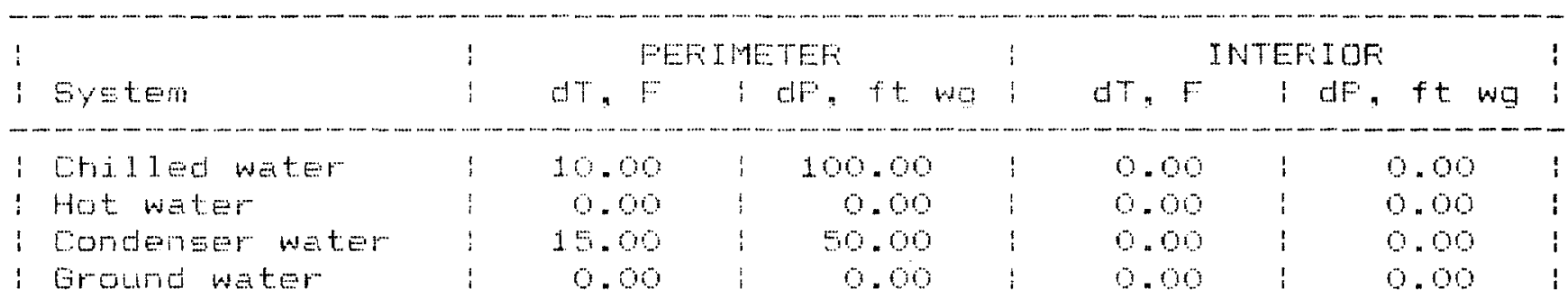

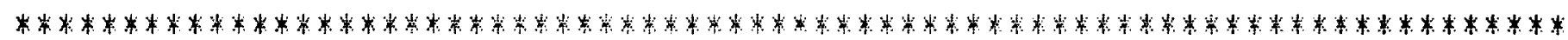

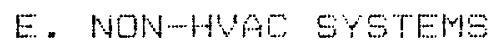

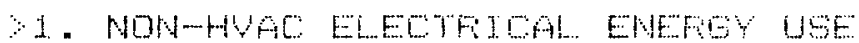

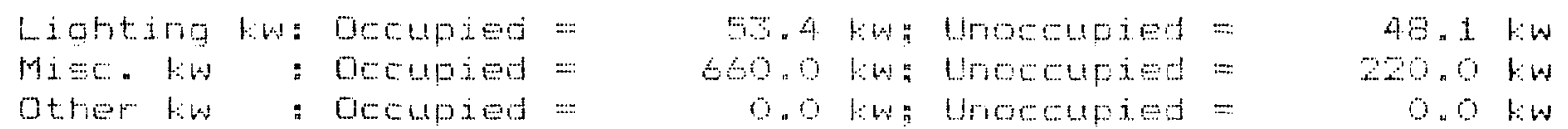

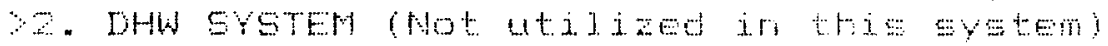

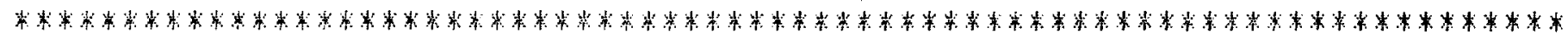
F. FUEL CISTS

\begin{tabular}{|c|c|c|c|c|c|c|c|c|c|}
\hline$\because 1$. & 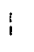 & \multicolumn{3}{|c|}{$F=i \omega d$} & $\vdots$ & GTHEF FUELB & ! & Unit & {$[0=t$} \\
\hline ELECTFICALI & 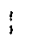 & Occupied & i & Unoceupied & $\dot{t}$ & 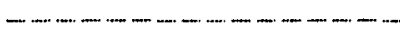 & & & $---\infty-\cdots$ \\
\hline ENEFGY USE & $i$ & $($ oftan $)$ & $!$ & (a) (lot) & : & iNat:Ural Gas & i & $\Pi=$ & क/therm \\
\hline$-\cdots-m-m-\infty-m$ & -7 & $---\cdots-m-n$ & -...- & (2) & - & IFUE] Gil & $i$ & $\Pi / \mathrm{a}$ & \$/U.S.9a1 \\
\hline Compressor & $i$ & 0.050 & 1 & 0.050 & $i$ & iFroperie & ; & $n / a$ & $\$ 1 \mathrm{~b}$ \\
\hline Fesistance & 1 & 0.050 & i & 0.056 & $i$ & ifiemote Heating & $i$ & $n / a$ & \$ /METU \\
\hline Inductive & $!$ & 0.050 & ! & 6.050 & $i$ & fremote cooling & $i$ & $n / 3$ & $\$$ MBTU \\
\hline
\end{tabular}


TOTAL BUTLDING ENEFEY SURHAFY

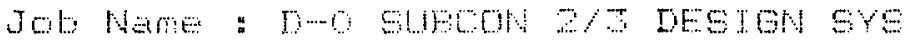

Buidong Neme : D-o sueconstruct $2 / 3$ (Complex)

Site : AUROFA, IL FEFil : User Detined

Dete: $: 1-16-8 \%$

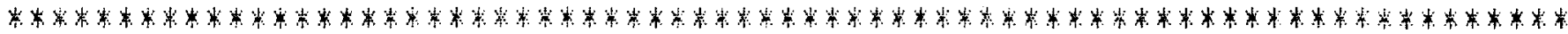

HVAC ENEFGY

Alinilat

Cost

Component

$\left(\$ / y^{-}\right)$

Electric (Oce)

Electrie (Unoce)

Natural Gas

Fue 1 Q1

Fropane

Femote Heatirig

Fiemote Cooling

HUAC TOtal

NON-HVAC ENEFOY

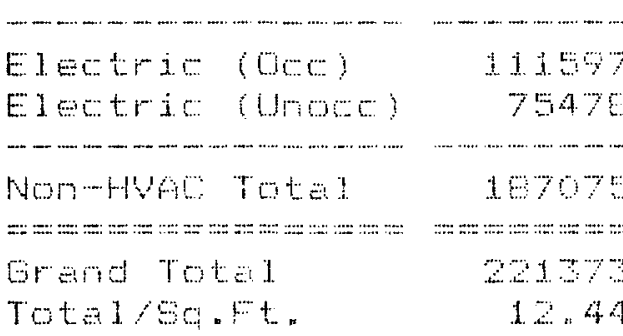

Emeroy or Fuel

Units DOE FUF DOE FIF

consumed

( 1000 ETU) (1000 BTU)

1956

14943

0

0

o

0

34298

$4490540 \quad 1.21225$

$3466692 \quad 1019985$

0

0

0

0

0

7957282

2341210
Feriod : Al

Zorie a EJot:

Serial Number: 60117662.0

387115 r:wh/yr

29885 fwh yr

o therms/yr

o U.S. qa1/yr

o $1 \mathrm{~b} / \mathrm{yr}$

$0 \mathrm{METL} / Y$ H

O METU/Yr

281944 fwh
150955 bwhy

$761762 \%$

HVă Summery:

Hvac Total

Total Hea Electoma Eneroy

Non Eleetriced HUE Eroroy =

rey:

\begin{tabular}{|c|c|}
\hline $1 \ln$ & $=11600$ ETU RUF \\
\hline i therm net 0 as & $=100000$ eTu PUF and FrF \\
\hline U. 5.9 .1 oil & $=198700$ eTU NUF and DTF \\
\hline Inp. as & $16800 \mathrm{Q}$ BU FUF and FTF \\
\hline 1 ] b propene & $=21680$ BTU FUF and FTF \\
\hline FIUF & $=$ Fesource utidization factor (Source value) \\
\hline$F: I F$ & $=$ Fesource impact factor (Foint of luse value) \\
\hline MEH & $=1000 \mathrm{BTU} / \mathrm{hr}$ \\
\hline METU & $=$ Million ETU \\
\hline
\end{tabular}


Job tene : D-O SUECOH $2 / 3$ DESIGN SYS

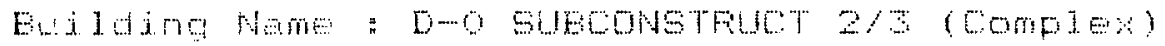

Fage 1

site : ALFORA IL FEFII I UEer Defined

Seope of Analyeis : Cooling amd Heatime Syetens

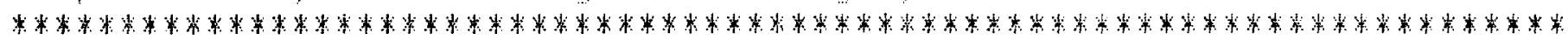

A. AIF HANDL ING SYSTEMS

(ANULAL FAN OFEFATING EQET =

$\$ 10.50)$

Are perimeter and interior zone on seme stpply fan 74

1. FEFIMETEF TEFITNAL TYFE: "CONSTANT VOLUAE

Supply fan type: Forward curved

Supply fam total static pressure = 2.0O in. Wo.

Feturn fan type : Controlled pitch axial

Feturn fan total static pressure $=1.00$ in. wa.

Design supply eir= $36000 \mathrm{cfm}$ o $55 \mathrm{~F}$ : Vent. air= $600 \mathrm{efm}$

Are cooling terminals used for heating ? Yे

Economizer type : Integrated, enthalpy controlled

$I=$ a ventilation reclaim device used? $\mathrm{N}$

fre ventilation air dempers closed for unocoupied periods? end

2. INTEFIOF TEFHINAL TYFE " CONGTANT YOLUME

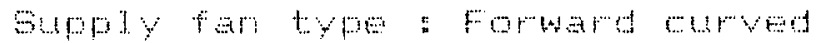

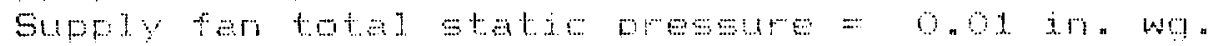

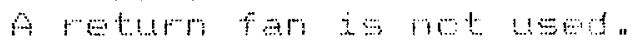

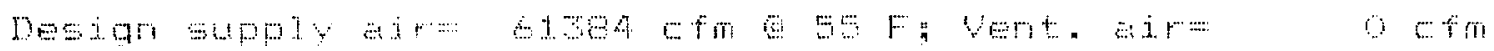

fri enomizer is rots

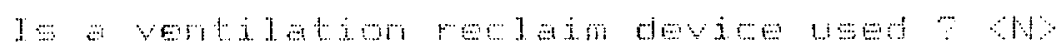

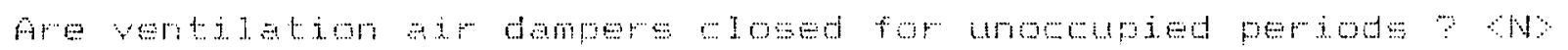

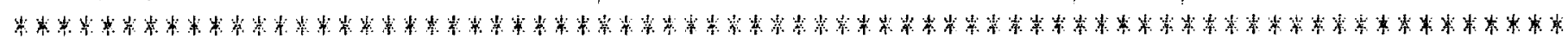

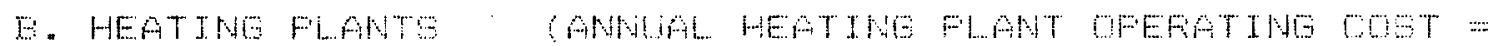

$\$ 0)$

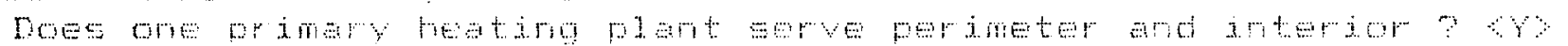

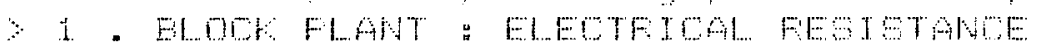

$\mathrm{I}=$ hydrom heatino asod? $\mathrm{NB}$

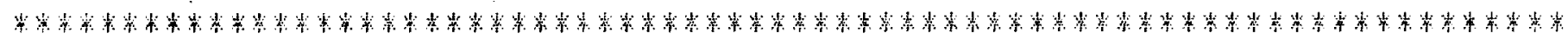


Job Name: D-o SUECON $2 /$ DESTH $5 Y$

Euilding Nane : Dmo Subonstruet $2 / 3$ (Complex)

Fage 2

Site : AUFOFA, IL FEFMI, WeEr Defined

Scope of Anelysi= " Cooling ard Heating Systene

Dete: $: 11-16-87$

60117962.0

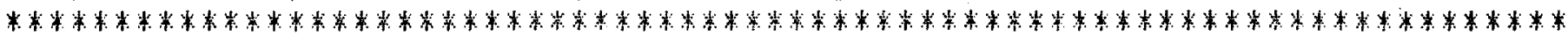

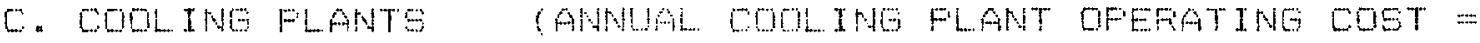

$\$ 15,564)$

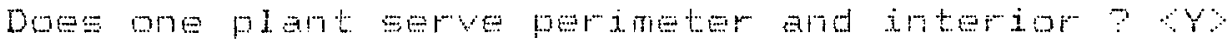

1. ELOCF FLANT: FECIFFOCATINE WATEF COOLED

Capacity $985 \mathrm{~F}$ entering water $=230.0$ tons

rw/Ton 9 s F entering water $=0.85$ lw/ton

Is rydronic cooling used? CY?

$I \equiv$ chilled water reset Leed $P$ No

IE hot gas bypese used 7 N

Is condenser performance altitude adjusted ? Yे

Is there one compreseor per condenser circuit ? $\mathrm{Y}$

Are compressors cycled $?$ ry

Heat sint: type " open cooling tower

Minjmum entering water temperatum

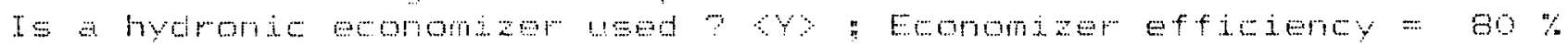

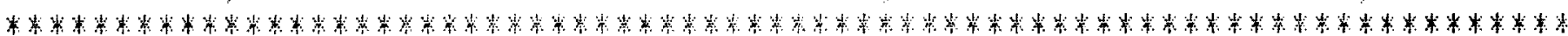

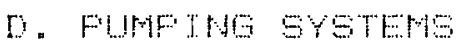

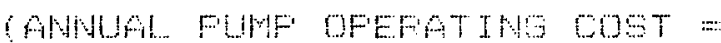

to 415

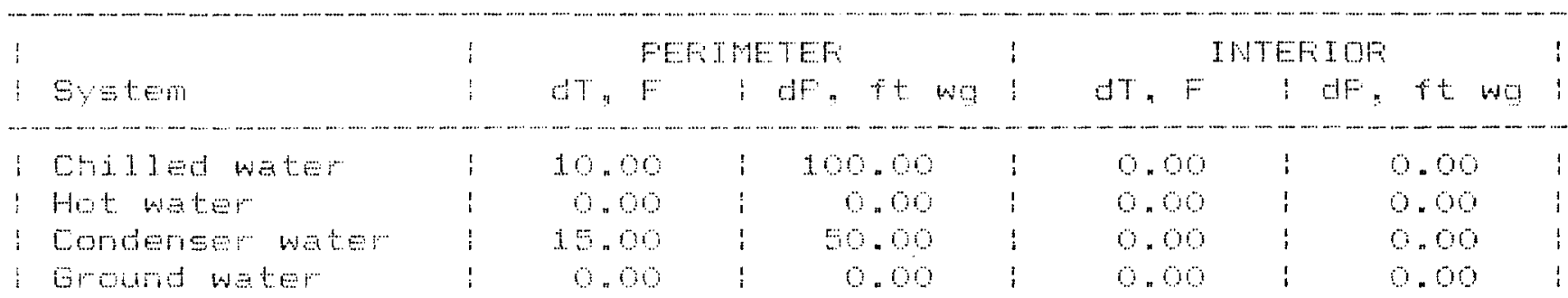

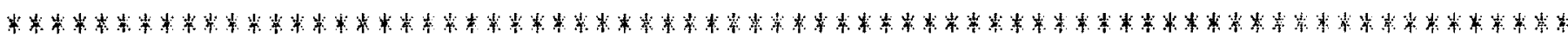

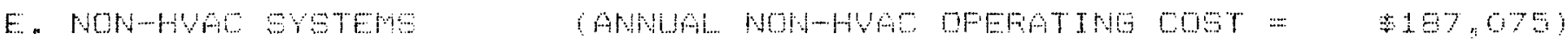

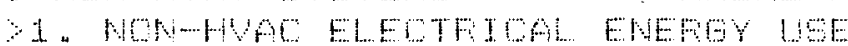

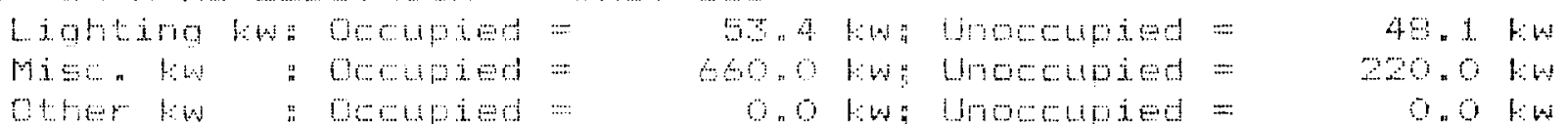

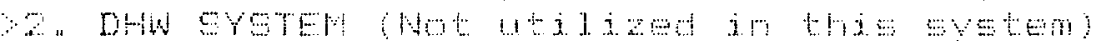

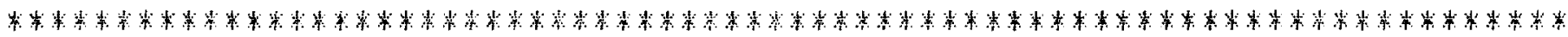

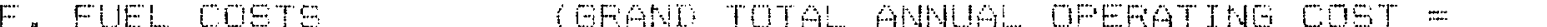

\begin{tabular}{|c|c|c|c|c|c|c|c|}
\hline $1 "$ & 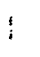 & \multicolumn{2}{|c|}{ Ferdod } & i & Z. OTHEF FUELS & $i$ & Und $\cos =\mathrm{t}$ \\
\hline ELECTEIGAL & $i$ & Octoied i & Unomenpied & $i$ & 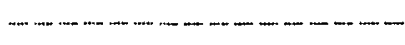 & & ....... \\
\hline ENEFGY USE & 1 & 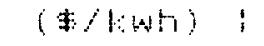 & $($ statis) & $i$ & Batlura1 Gas & 1 & $m$ a $\$$ / therm \\
\hline$-m--m-n-m$ & $-\cdots$ & 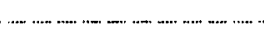 & 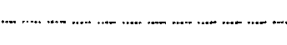 & $-\cdots$ & ifuEl. Oi 1 & $i$ & $\$ / U .5 .0 \mathrm{al}$ \\
\hline Compressor & $i$ & 0.050 & 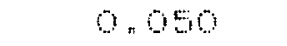 & 1 & Fropane & 1 & 和 \\
\hline Fes $i=\tan :$ & $i$ & 0.050 & 0.050 & $i$ & AFemote Heating & $i$ & \$ABTU \\
\hline Inductive & $\vdots$ & 0.05 & 0.050 & $i$ & iFenote Coojing & $i$ & $\Pi / a$ S/METU \\
\hline
\end{tabular}




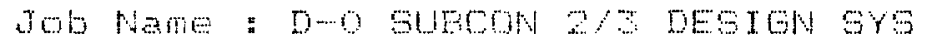

Eudiding Name: D-O SUECONeTfuCT $2 / 3$ (Complex)

Site Name a AUFOFA al FEFMI User Defined
Dete: $11-16-87$

Serial Number" 60117852.0

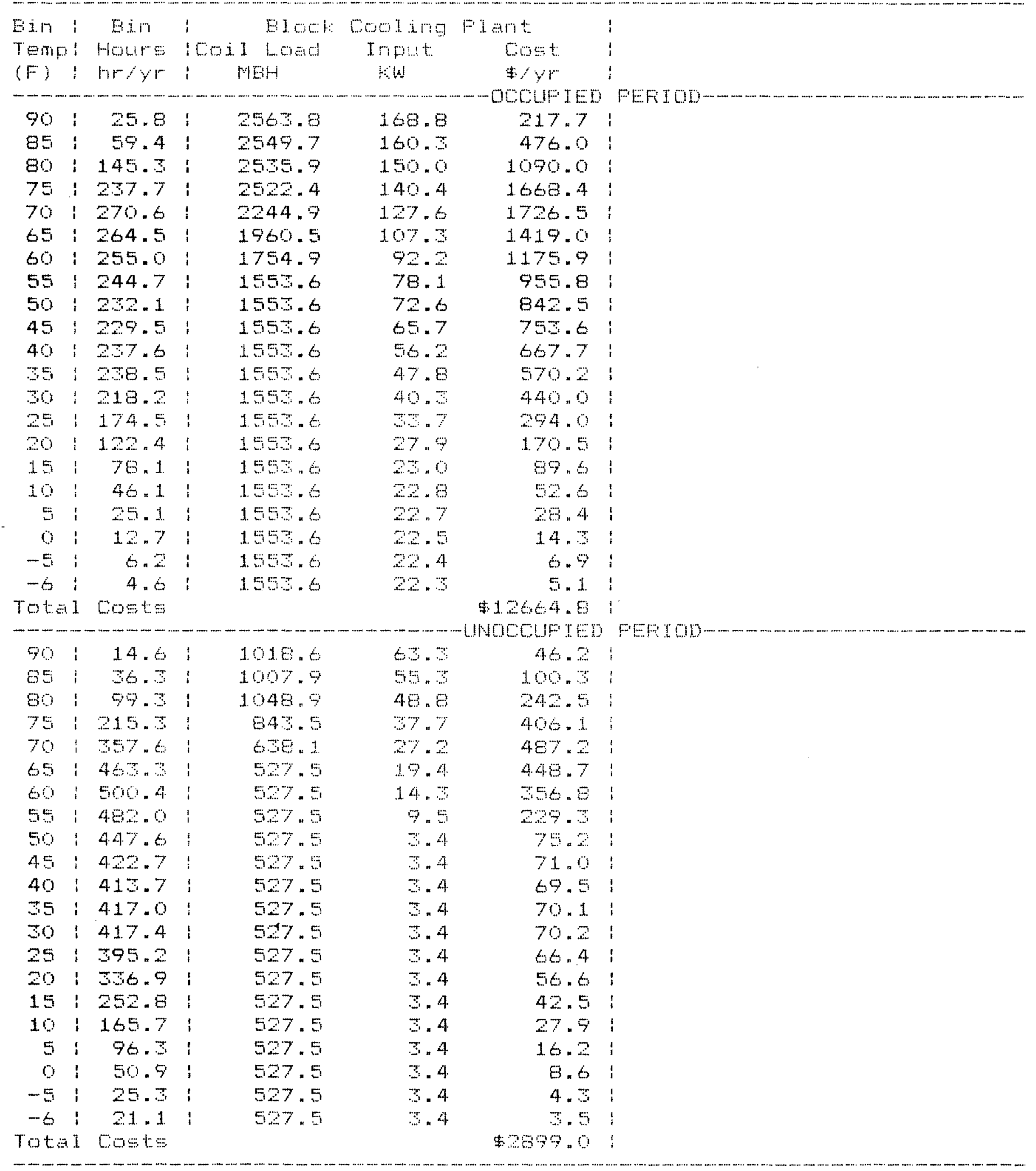


BLOCE HEATING FLANT COSTS

Job Name: Dmo SUECOH $2 /$ DESTON SYS

Building Nane : 0 o surounstruct $2 / \mathrm{s}$ (Complex)

Site Hane: AUFOFA, IL FEFMI, Usew Defaned

Date: $11-16-87$

Serial Number: 60117832.0

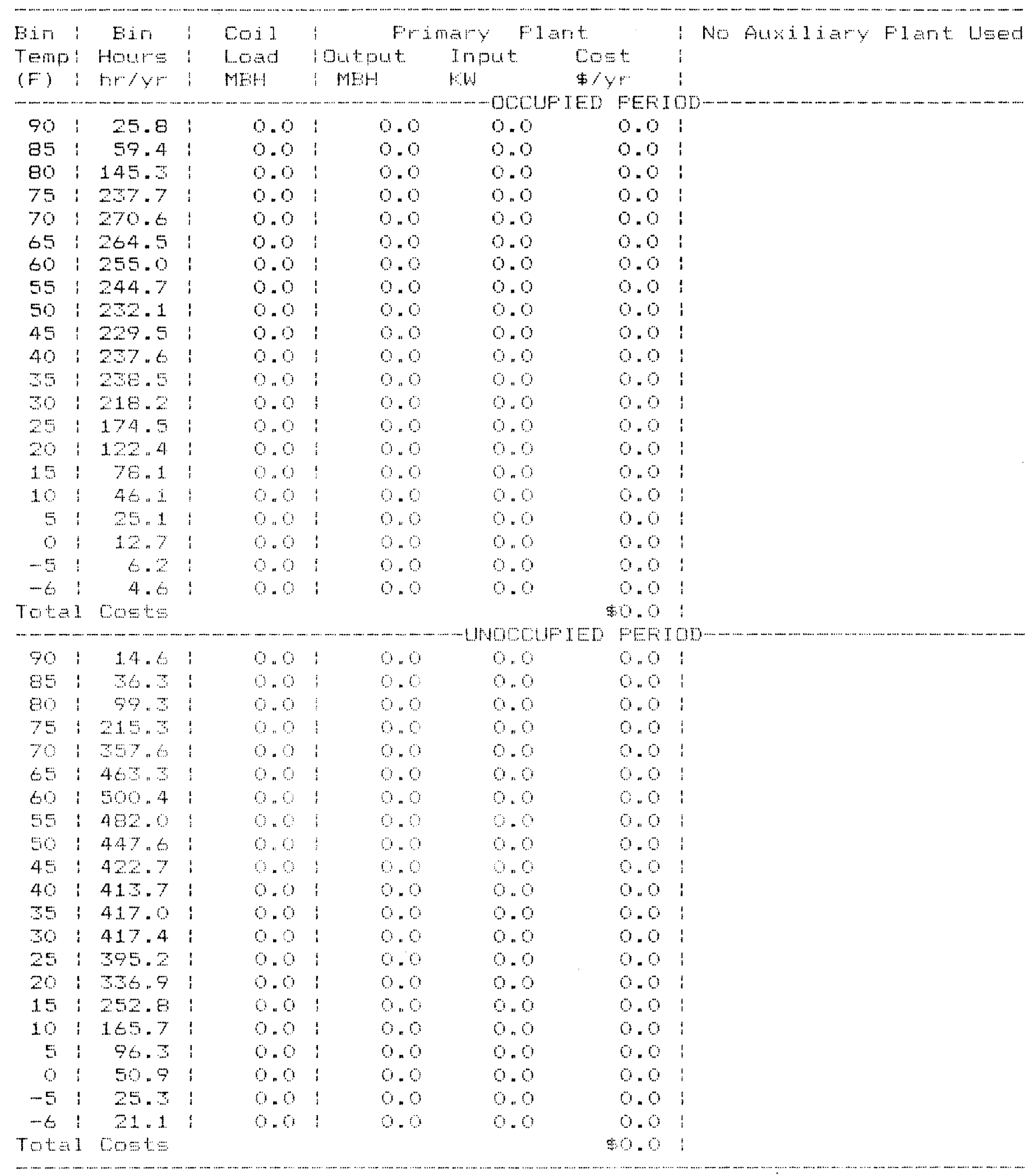


Job Nane : D-O SUECON $2 \%$ DESIGH SY

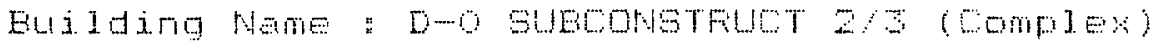

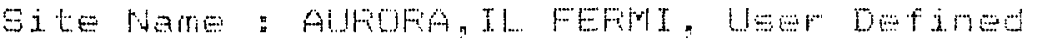

Date : $11-16-87$

Seria I Number:

611782.0

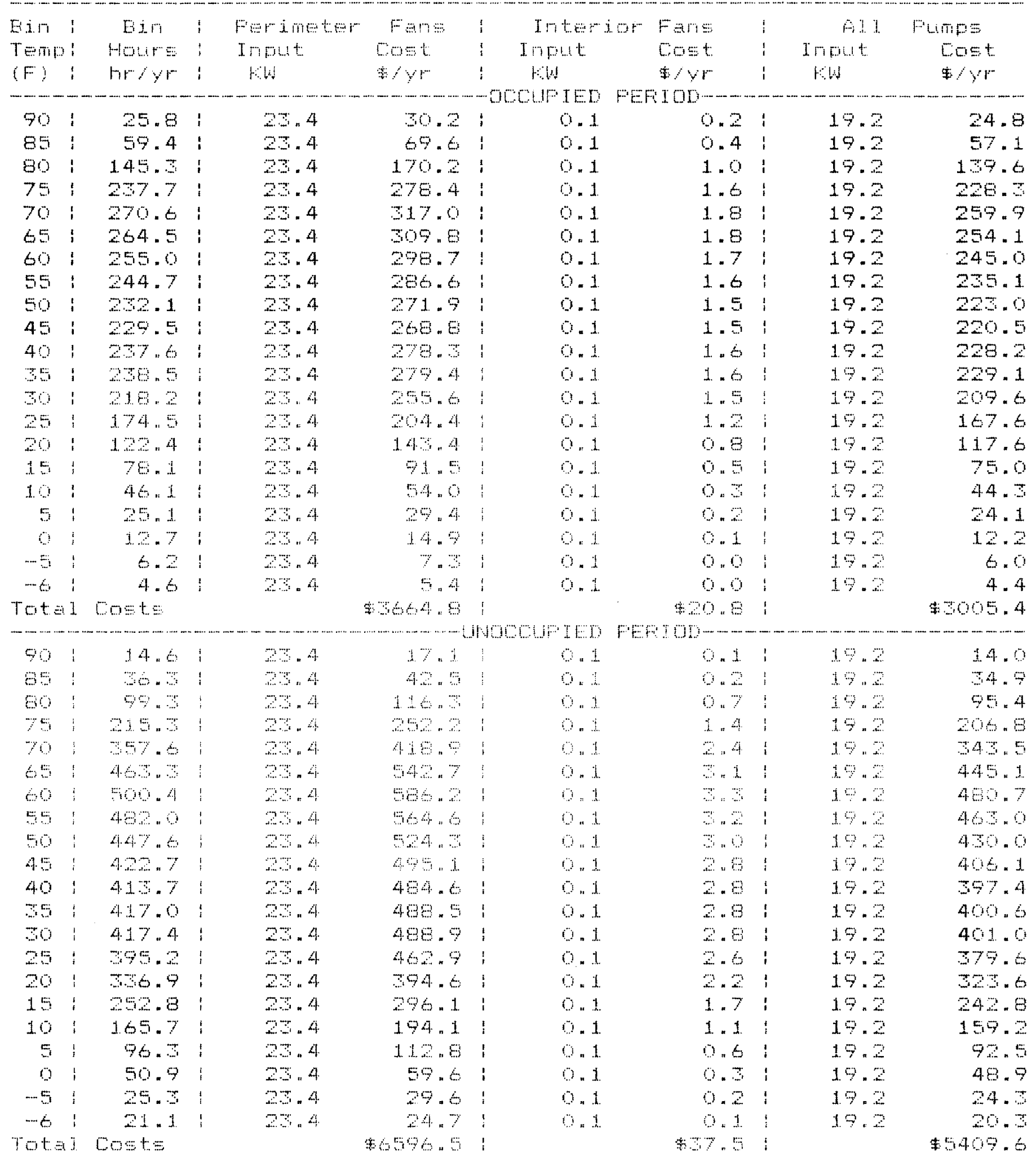




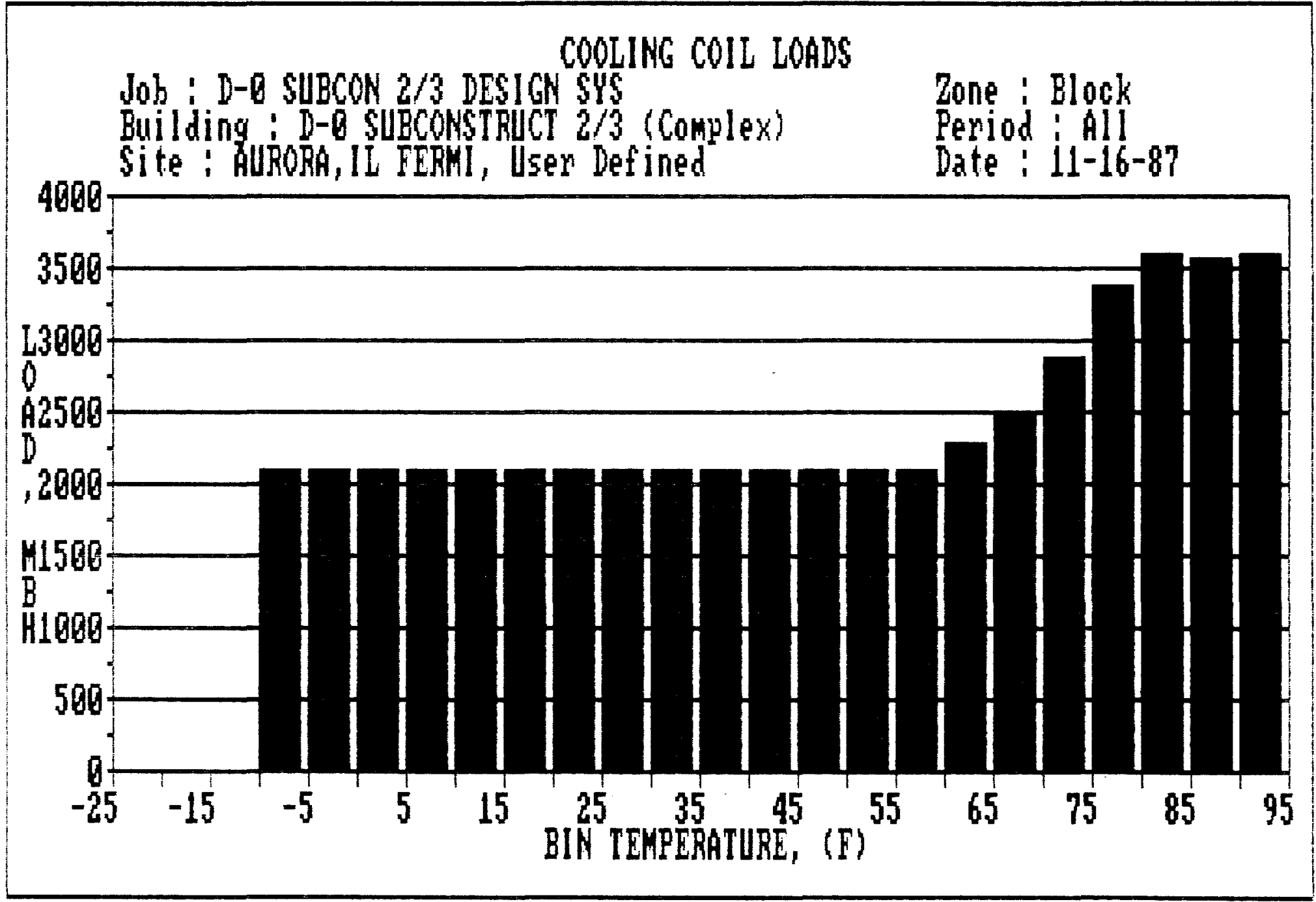




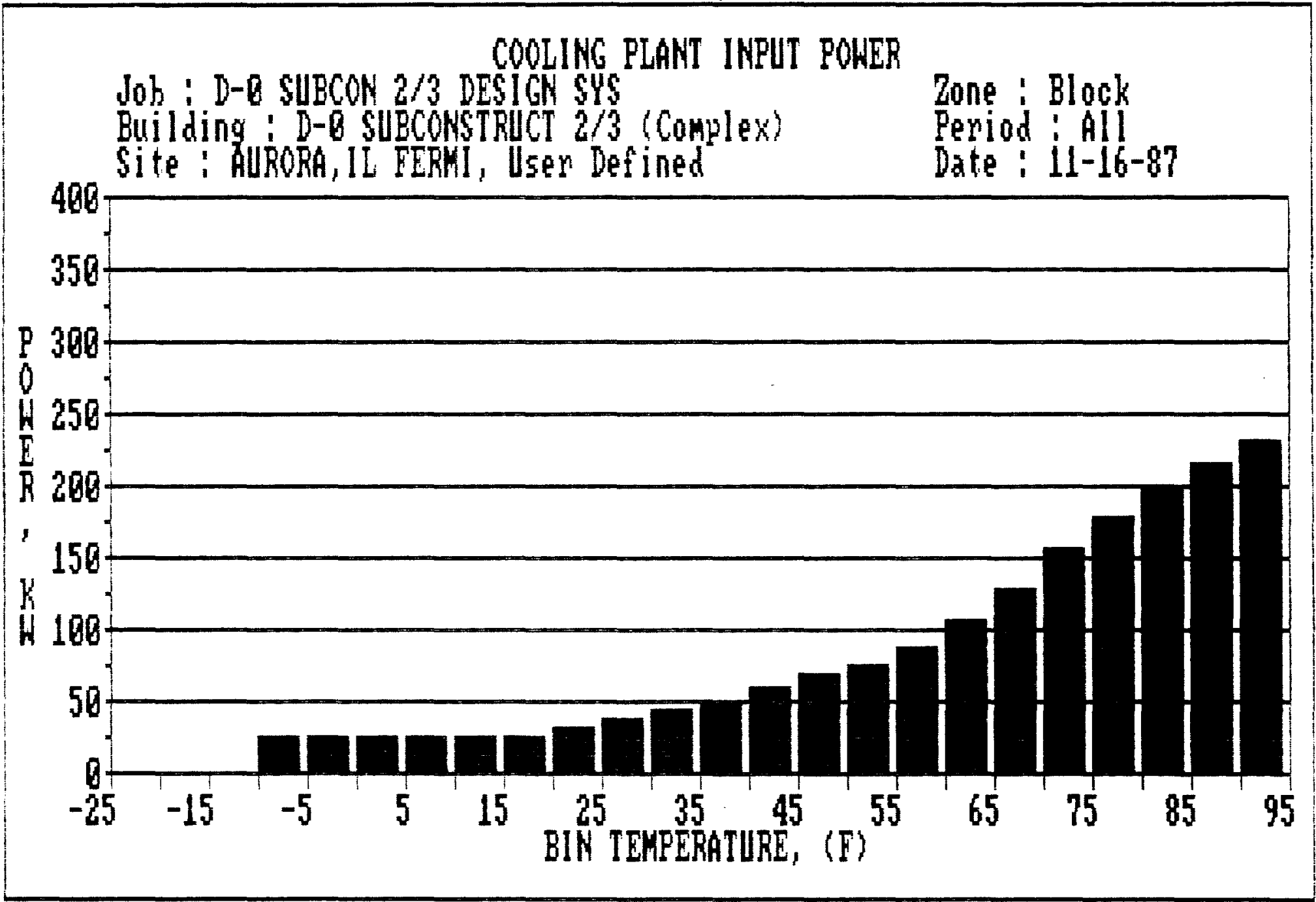




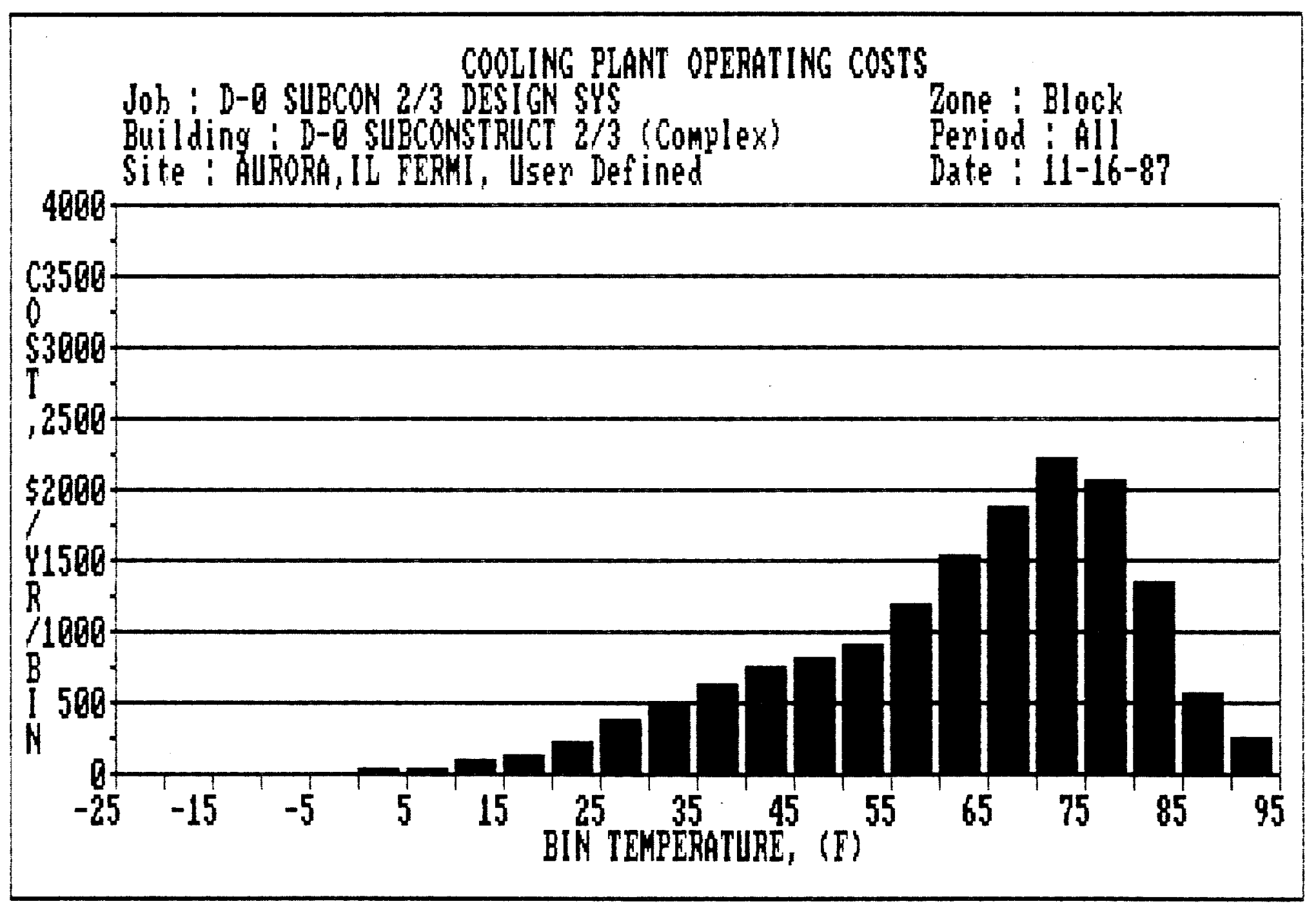




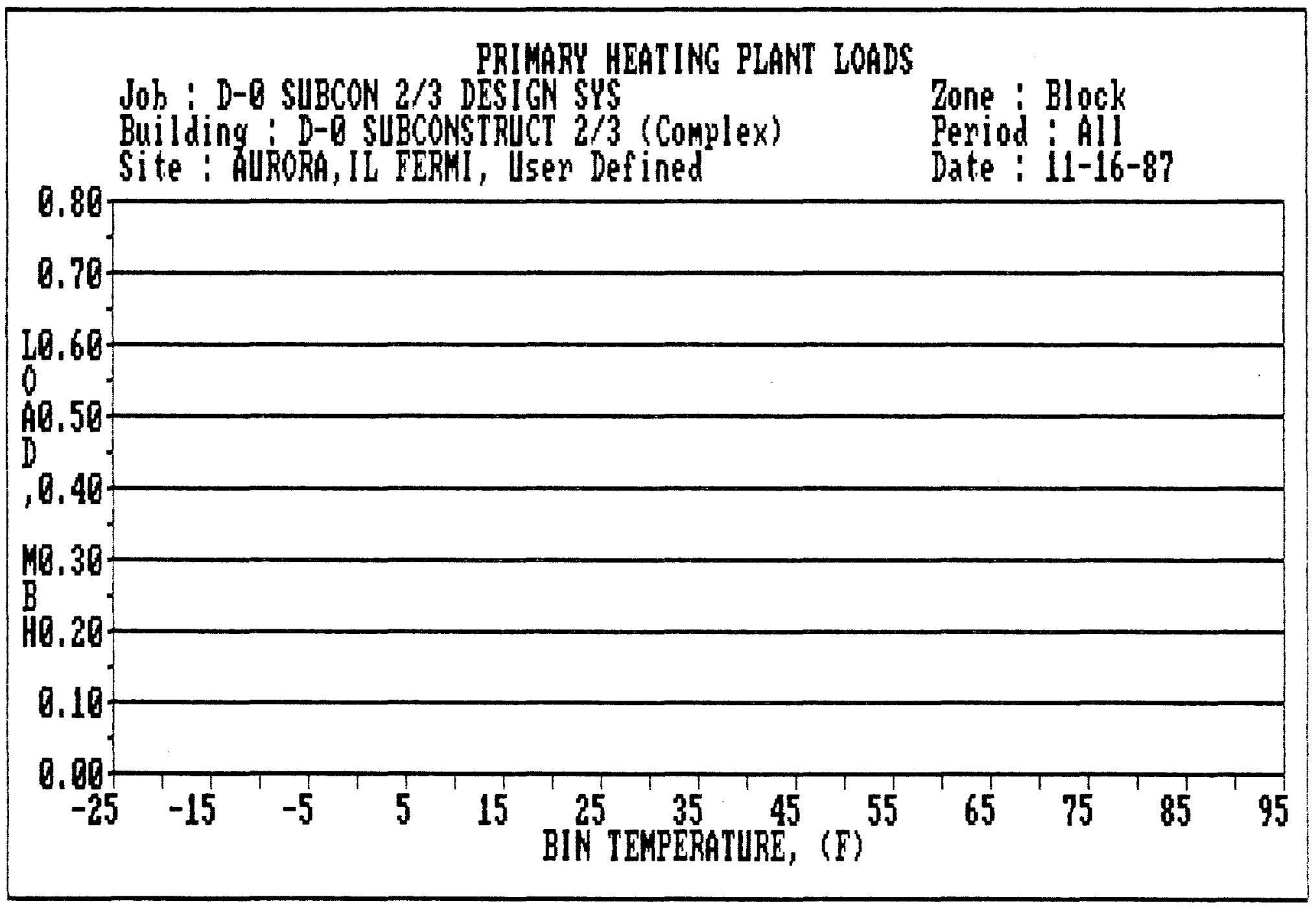




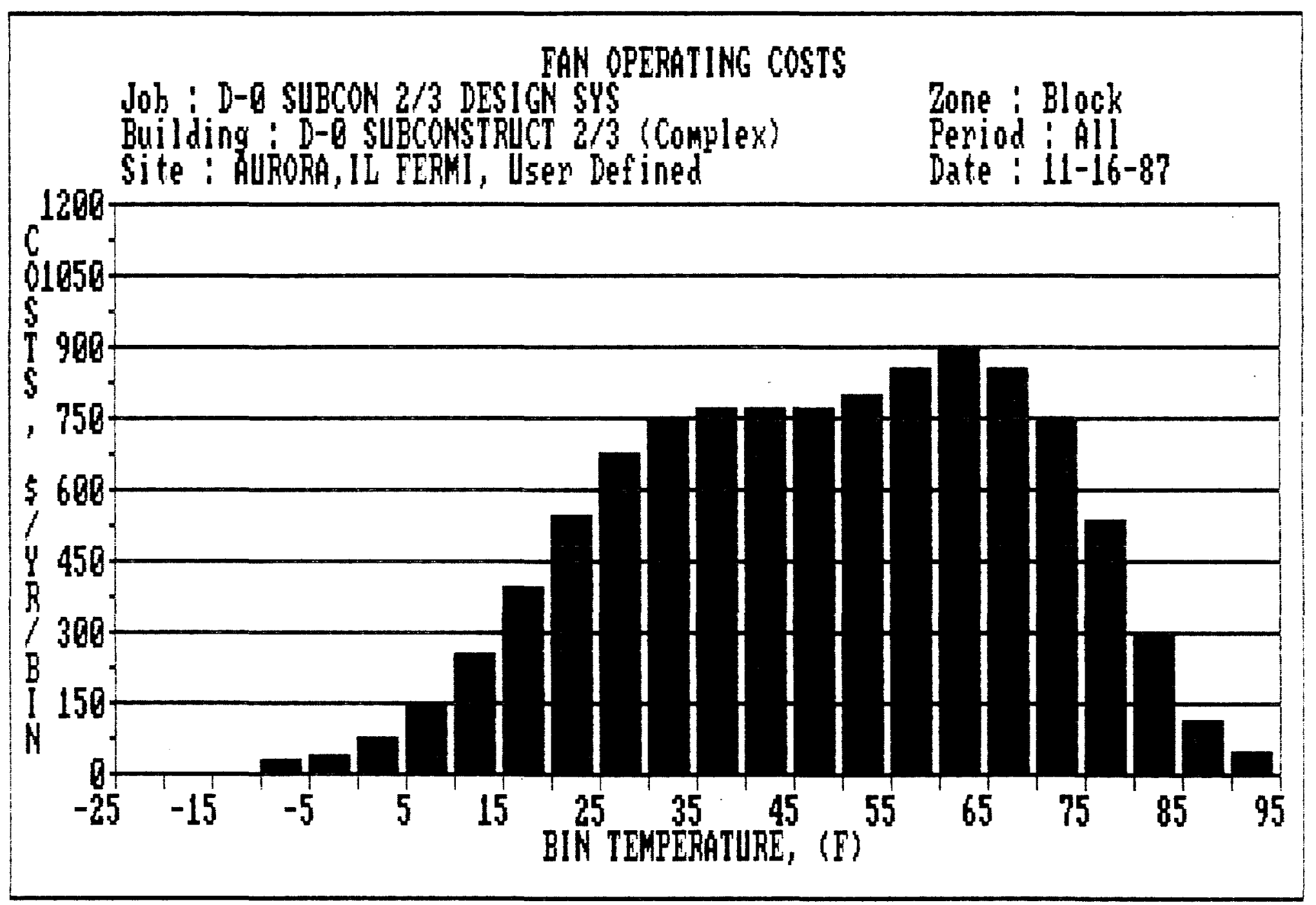




\section{$\mathrm{V}-\mathrm{c} .-2$.}

FEFMLAE D-O EXFEMIMENTAL FAEILITY

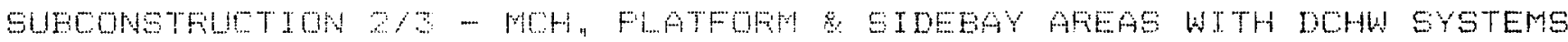
OFEFAT TONAL FEFFOFIANEE STWLILATIOH

ALTEFNATE COMFAFIOUN EYETEM NUMEEA 1

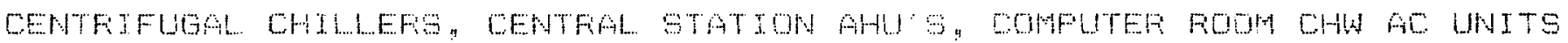
AND WATEF COOLED ELEOTFOHTC FACHS

WTTH THESE ENEEGQ FEATUFE:

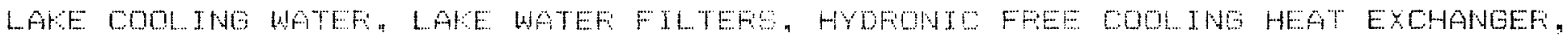

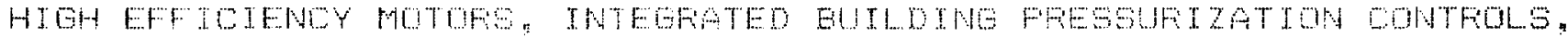

AUTOHATTE LAKE WATEF FUTF STAOMNG INTEGATED ENTHALFY EODNOMIZEF,

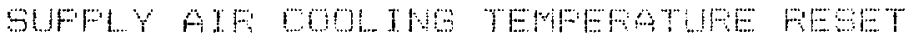

उ. OCTOBEक $19 \%$

ANALYS: S.F.GFTHLOUCH

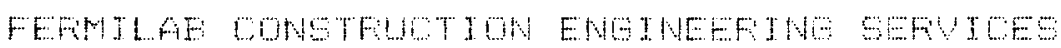


Job Name : D-O SUECON 2 / ALT 1998

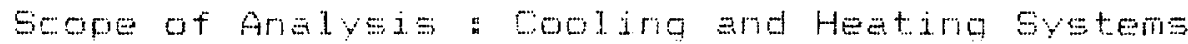

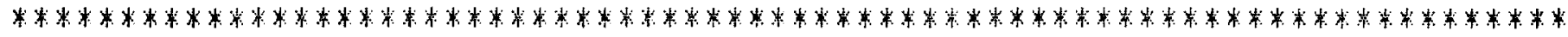

A. AIR HANDLING SYSTEM

Are perimeter and interior zone on same supply far $\because$ do

1. PEFIMETEF TEFMTNAL TYFE " CONSTANT VOLUME

SLPFly fan type : Forward curved

Supply fan total statie pressure = 2.00 in. wg.

Feturn fan type : Controlled pitch axial.

Feturn fan total stetic pressure $=1.00$ in. wg.

Design supply air $=36000 \mathrm{~cm}$ a $55 \mathrm{~F}$. Vent. air= $600 \mathrm{~cm}$

Are cooling terminals used for heating? $Y$ Y

Economizer type : Integrated enthalpy controlled

$I \equiv$ a ventilation reclaim device used? CN

Are ventilation a ir dampers closed for unoccupied periods 7 ha

2. INTEFITOF TEFMINAL TYFE : CONSTANT YOLUME

Supply fan type "Formard euryed

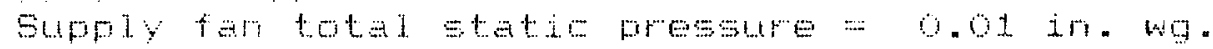

A return tan $1=$ mot. uset.

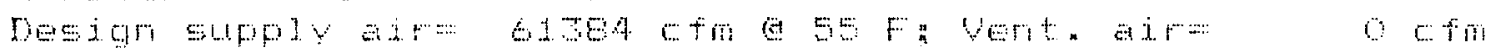

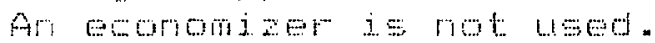

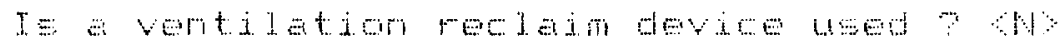

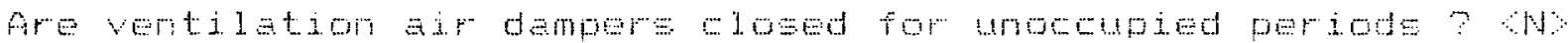

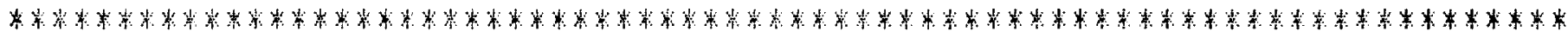
E. HEATTHE Fl...TH马

Does one primary heating plant serve perimetem and interior 7 ry

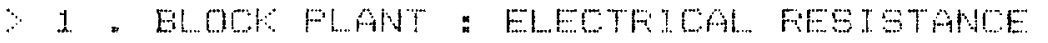

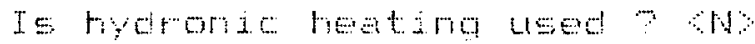

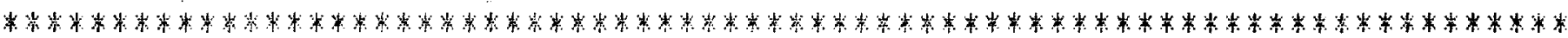




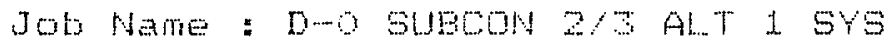

Fiage 2

Building Name : D-O SUECONSTFUET 2/3 (COMn]ex)

Date : $11-16-87$

Site : AUFOAM,TL FEFMT, USEr Defingd 60117862.0

Seope of Amaly $y=$ : Coding and Heating systems

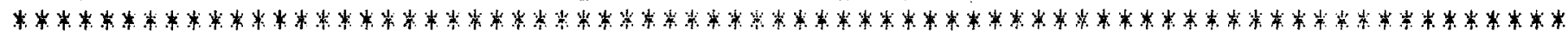

C. COOL ING FIANTS

Does one plent serve perimeter and interior ? Yy

3. ELOCH: FLANT : CENTFIFUGAL WATEF COOLED CHILLEF

Capacity a $85 \mathrm{~F}$ entering Hater $=230.0$ tons

rw/Ton g $85 \mathrm{~F}$ entering water $=0.78 \mathrm{kw} / \mathrm{ton}$

Is hydronic cooling Läd $? Y$

Is chilled weter reset LSEd ? YN

Is condenser performante altitude adjusted $? \quad Y \%$

Heat sint: type : Open cooling tower

Mininum entering water temperature = $2 \mathrm{~F}$

Is a hydronic ecomonizer used $?$ yY " Economizer efficiency = 80\%

Number of sequenced chillers=: 2

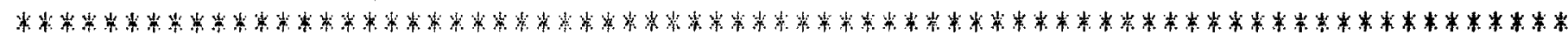

D. FUHFTMG SYETEME

\begin{tabular}{|c|c|c|c|c|c|c|c|c|c|}
\hline \multirow{2}{*}{3} & & $i$ & \multicolumn{3}{|c|}{ PEFMETEF } & 1 & \multicolumn{3}{|c|}{ INTEFIOF } \\
\hline & $8 y=\operatorname{ten}$ & 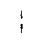 & $d i: F$ & $i$ & $d F, T+4 a$ & $!$ & $d i, F$ & 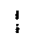 & ft. $\omega g$ \\
\hline & CtiII I Eo wate & $i$ & 19.0 & $i$ & 100.90 & 1 & 0.00 & $\stackrel{1}{3}$ & 0.00 \\
\hline & Hot watei & $\vdots$ & 0,0 & $i$ & 0.0 & i & 0.00 & $!$ & 0.00 \\
\hline & Conderiser water & $\vdots$ & 10.00 & $i$ & 50.0 & 1 & 0.00 & $j$ & $0=00$ \\
\hline & Groumd water & $i$ & $\mathrm{OH}$ & $!$ & 0.00 & 3 & 0.0 & $i$ & 0.00 \\
\hline
\end{tabular}

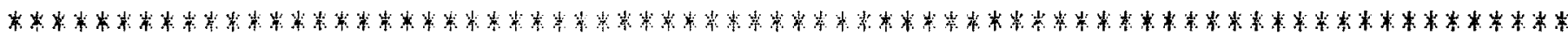

E. WOM-HYG $5 \mathrm{TSTES}$

1. NON-HUC ELEOTFIOA ENEWOY UE

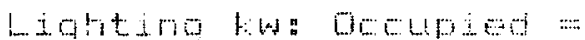
$5{ }_{2}+$ Ku: Uromoroded=
$4.1 \%$
Mison, $1: 4$ : Orouplew =

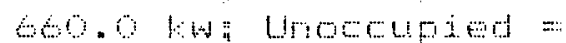

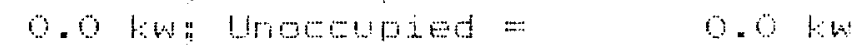
20.

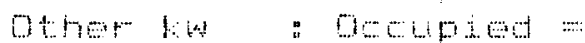

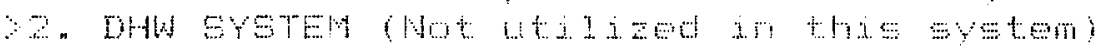

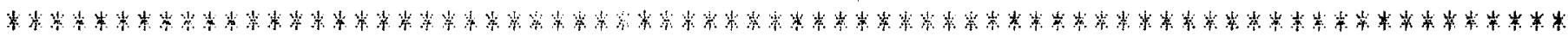
F. FUEL EOSTO

\begin{tabular}{|c|c|c|c|c|c|c|c|c|}
\hline$\because 1$. & $\mathrm{i}$ & \multicolumn{3}{|c|}{$F e r i o d$} & 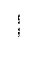 & a ombl futLs & 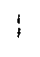 & Whit $-00 t$ \\
\hline ELETTACAL & 1 & $00 t+1 e d$ & 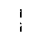 & 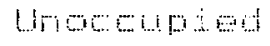 & 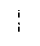 & 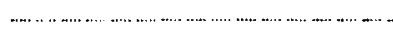 & & (- \\
\hline ENERGY USE & 3 & $(\$) \tan ()$ & $i$ & (towtio & $i$ & Matura $\mathbf{a g}=$ & $\vdots$ & na $a$ therm \\
\hline 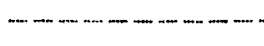 & $-\cdots$ & 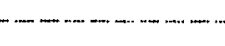 & $-\cdots$ & 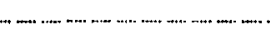 & ...... & [FuE] oi] & $i$ & $n / a \$ / U .5 .9 a 1$ \\
\hline Compressogr & $!$ & 0.05 & $!$ & 0.050 & 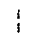 & :Foprie & 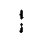 & $n / z$ 制 $1 \mathrm{~b}$ \\
\hline Fesistance & i & 0.050 & $!$ & 0.00 & $i$ & Fienote Heating & i & חIA \$/METU \\
\hline Inductive & $i$ & 0.050 & $\vdots$ & 0.050 & $!$ & ifenote Coolino & $i$ & 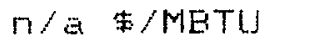 \\
\hline
\end{tabular}


TOTAL BUILOING ENEFGY BUMHAFY

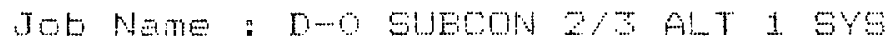

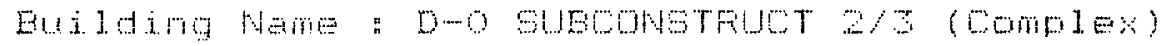

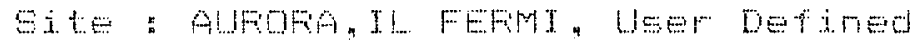

Ferigd:Al1

zome : Diot

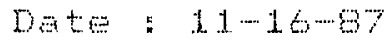

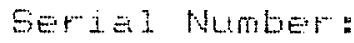

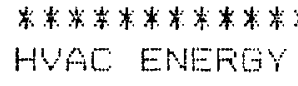

Comporant

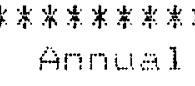

$\operatorname{mos}=\mathrm{t}$

Energy or Fuel

(t) $\left.y^{-}\right)$

Units $t=$

Corgumed

DOE FUF

DOE FIF

$--\cdots-\cdots-\cdots-\cdots$

\begin{tabular}{|c|c|c|}
\hline 21736 & 474726 & folut $>$ Y \\
\hline 16486 & 29717 & fwh \\
\hline 0 & 0 & therms $/ y^{-}$ \\
\hline ) & 0 & U.5. qa1,y \\
\hline 0 & ? & $1 \mathrm{~b} / \mathrm{vr}$ \\
\hline 8 & 0 & MATU $Y$ Y \\
\hline 0 & 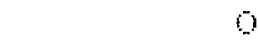 & METU/Yr \\
\hline
\end{tabular}

(1000 ETU)

Electrie (OCE)

4.4726 kwh/vr

5042826

(100) ETU)

Electric (Unoce)

Natural Ges

Fle 1 oj.

Fropane

Fenote Heating

Femote Cooling

HVAC Tote1.

5322

3624742

1. $48 \div 72$

1125.31

0

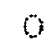

5

i)

)

0
8867568

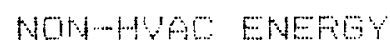

\begin{tabular}{|c|c|}
\hline electric & $(0<)$ \\
\hline E deotric & (UTOE) \\
\hline
\end{tabular}

\section{Momplab rot.s.}

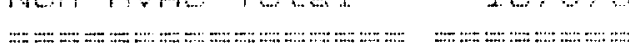

\begin{tabular}{|c|c|}
\hline 111597 & $22 y+94$ fwh y \\
\hline 7948 & 190950 lwh y \\
\hline
\end{tabular}

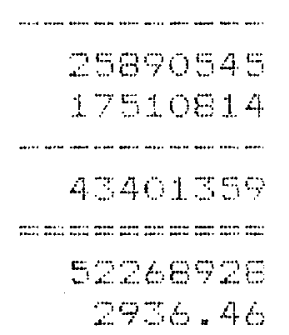

761720
515204
1276979
1579780
86298

orame Total
Totelsomt.

$2,5,5$

13.6

206.46

66.98

MOG Summa'
Hen $70+a 1 \quad \mathrm{Co}=$
2.15 159 . Ft.

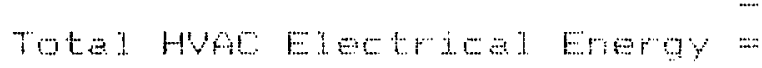
$16.97 \%$ of 7otal wost
The: 4. 95 twh/so.Ft. $/ Y$ r

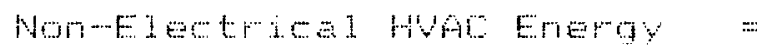
$16.97 \%$ of Total EIEctirimal Emergy $0.00 \%$ ot Total Energy

$t=y$

\begin{tabular}{|c|c|}
\hline $1 \quad F i+i=$ & $=1160$ ETU FUF : \\
\hline 1 them & $=1000 \mathrm{AO}$ DTL FUF and FIF \\
\hline U. 5.931011 & 190700 IUU FUF \\
\hline 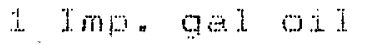 & and Fif \\
\hline It propane & 2. 680 FTU FUU and FIF \\
\hline FILF & Fiesource utilization factor (Gource value) \\
\hline FIIF & $=$ Fesouroe impact factor (Foint of use value) \\
\hline MEH & $=1000$ ETU/hr \\
\hline FETU & $=$ Million ETU \\
\hline
\end{tabular}




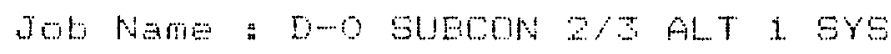

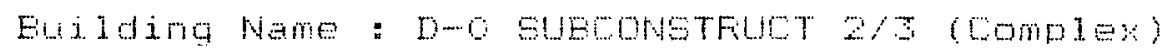

Site : AUFCFA, IL FEFMI, UEer Defined

Soope or Analysis a Cooling and Heating bytems

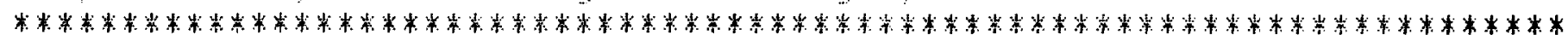

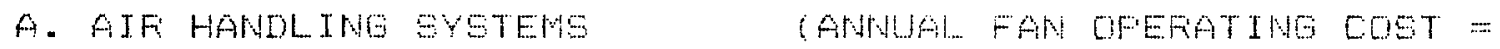

Are perjmeter and interior ane on ane anpoly fan $?$

1. FEFIMETEF TEFIMAL TYFE a COHETANT VOLUME

Supply fan type : Forward curved

Supply fan total static pressure $=2.00$ in. 10.

Feturn fan type: Control]ed pitch axial

Fieturn fan total static pressure $=1.00$ in. wg.

Desjgn supply air= 36000 Efm E Ss Fa Vent. air= bou cfm

Are cooling terminals used for heating ? ry

Economizer type : Integrated, enthalpy controlled

Is a ventilation reclain device used $?$ hy

Are ventilation air dampers closed for unoccupied periods $?$ No

2. INTEFIOFF TEFIMTNAL TYFE : CONSTANT VOLUME

Supply tan type a Frward curved

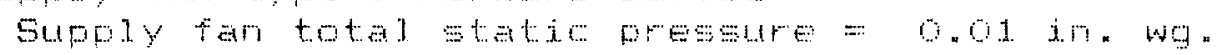

A return tar de not

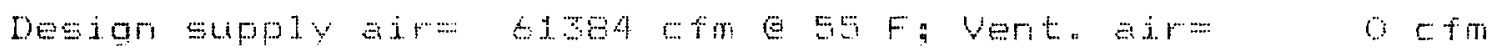

An womomizer is

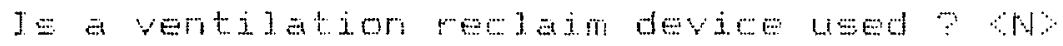

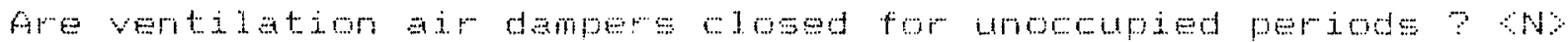

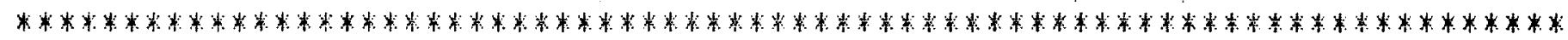

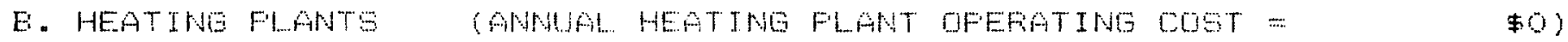

Does one primary heating want serve perimeter and irterior ? ry

1. BIOCH FIANT " ELECTFICAL FESISTANEE

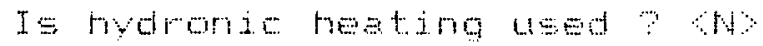

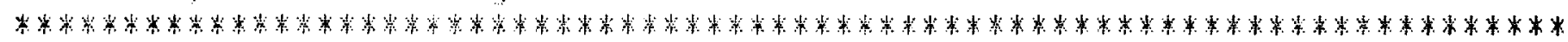


Iob Name : D-O SUBCON 2/2 ALT 1 SYS

Fage 2

Euilding Nane : L-6 SuedonstFuct $2 / 3$ (Complex)

Dete: $11-16-87$

Site: AlFORA, IL FEFH :USer Defined

60117862.0

Scope of Analyede : Cooldio and Heating Syetems

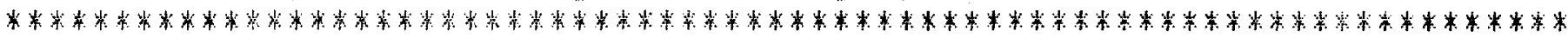

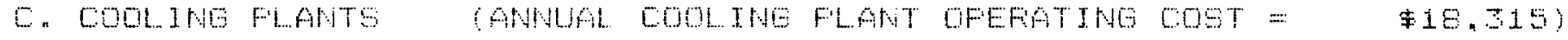

Does one plant eerve perimeter and interior ? Cy

1. ELOCF FLANT : CENTFIFUGAL WATEF COOLED CHILLEF

Capacity \& $35 \mathrm{~F}$ entering water = 230.0 tons

rw/Ton $85 \mathrm{~F}$ entering water $=0.78 \mathrm{kw} / \mathrm{ton}$

Is hydronie cooling used $?$ Yे

Is chilled water reset used $?$ N

Is condenser performance eltitude adjusted ? y

Heat sink type : Open cooling tower"

Minimum entering water temperature $=32 \mathrm{~F}$

Is a hydronic economizer used? Yy ; Economizer efficiency $=80 \%$

Number of sequenced ohiliers $=2$

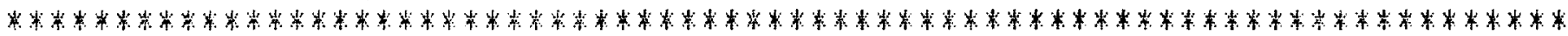

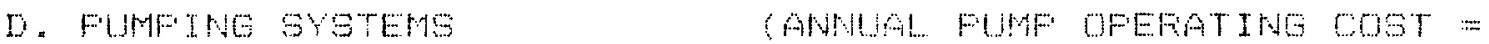

\$. 587 )

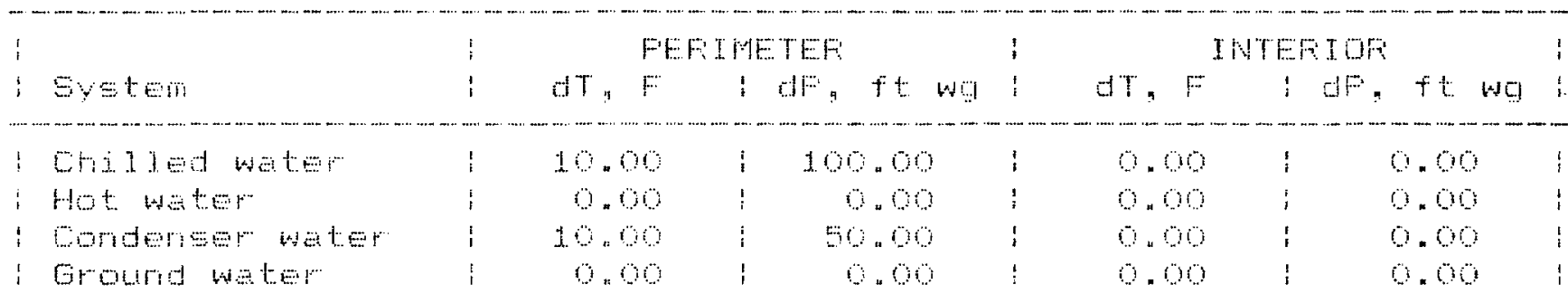

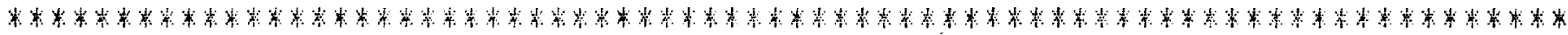

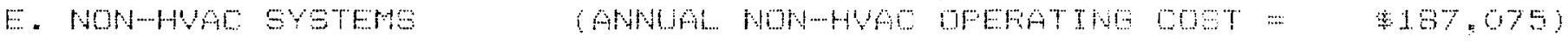

1. NON-HOAO ELECTFIOAL ENEFEY USE
L.

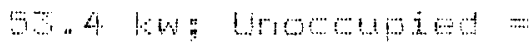
$49,1.16$

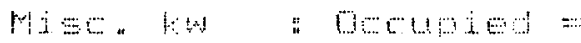
ém
$20,0 \quad 10$
Dther

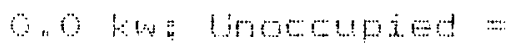
a $1 \therefore \ln$

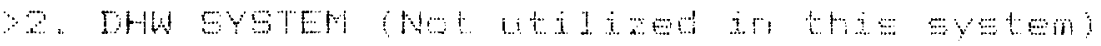

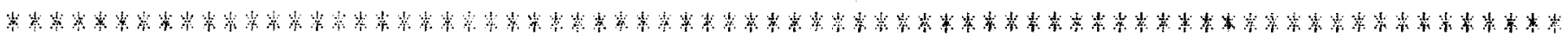

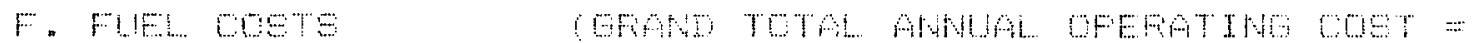

: $25,27 \%$

\begin{tabular}{|c|c|c|c|c|c|c|c|c|}
\hline$\because 1$. & $i$ & \multicolumn{3}{|c|}{ mind } & 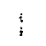 & $\because$ WTHE FUE & $i$ & bit $\cos =1$ \\
\hline wab]an & $i$ & 1) a. & $i$ & $4 m \omega \cdots+\cdots$ & $\vdots$ & 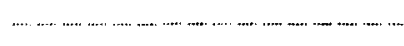 & 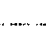 & 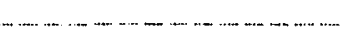 \\
\hline ENEFO 15 & $i$ & $(8)$ & $\vdots$ & $(t)$ (twi & : & Haturat $\mathrm{Ea}$ & $\dot{i}$ & $n y \quad+\tan m$ \\
\hline (n) & ...... & .................................... & $\ldots .$. & 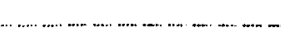 & ..... & ifuel Oil & 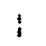 & tu. $\mathrm{t}, \mathrm{g}=1$ \\
\hline Compresesor & $i$ & oto & $i$ & 4.6 & $i$ & iFropane & : & Dit \\
\hline resistames & $i$ & का & $\vdots$ & 9.0 & $i$ & IFamote Heating & $i$ & W $Y$ HET \\
\hline Inductive & $!$ & 0.05 & i & 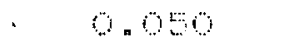 & $\vdots$ & iforote Condino & 3 & क METL \\
\hline
\end{tabular}


Jot hane" D-0 subCOn $2 / 3$ ALT 1 SYs

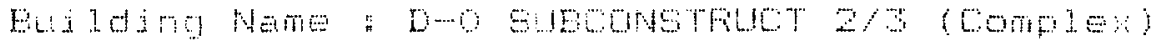

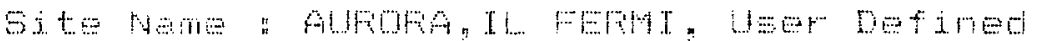

Pate $=1-16-67$

Serdan Number" 6012782.0

\begin{tabular}{|c|c|c|c|c|c|c|c|}
\hline 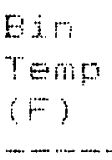 & $\begin{array}{c}i \\
\vdots \\
\vdots \\
\vdots\end{array}$ & $\begin{array}{l}\text { Bin } \\
\text { Hom } \\
\text { Hry }\end{array}$ & isis & $\begin{array}{l}\text { Elont } \\
\text { 1. Lomet } \\
\text { MEH }\end{array}$ & $\begin{array}{l}\text { oolimg } \\
\text { Imput } \\
\text { out }\end{array}$ & $\begin{array}{l}P=t \\
{[0=t} \\
5 / y\end{array}$ & $\begin{array}{c}i \\
\vdots \\
1\end{array}$ \\
\hline 90 & ! & 25.8 & i & 2563.6 & 159.2 & 205.3 & $i$ \\
\hline 85 & i & 59.4 & i & 2549.7 & 1.5 .0 & 45.4 .3 & ! \\
\hline 80 & $i$ & 145.3 & $\frac{1}{3}$ & 2535.9 & 144.6 & 1052.1 & $:$ \\
\hline 75 & : & 237.7 & i & 2522,4 & $13 \% 4$ & 1632.8 & i \\
\hline 70 & i & 270.6 & : & 2244.9 & 127.6 & 1726.0 & $i$ \\
\hline 65 & : & 264.5 & $i$ & 1960.5 & 112.0 & 1480.9 & i \\
\hline 60 & i & 255.0 & i & 1754.7 & 102.2 & $1503 \cdot 4$ & i \\
\hline 55 & $i$ & 244.7 & i & 155.6 & 94.3 & $115 \mathrm{~s}$ & i \\
\hline 50 & $i$ & 23,1 & ! & 155.6 & 92.1 & 1069.3 & $i$ \\
\hline 45 & $i$ & $29 \% .5$ & $i$ & 155.6 & 89.8 & 10007 & : \\
\hline 40 & $i$ & $277 \times 6$ & $i$ & 155.6 & $8 \omega_{.0}$ & 1021.6 & $i$ \\
\hline 5 & $i$ & 230 & $i$ & $156 \%$ & 64,0 & 72,9 & i \\
\hline 3 & $i$ & 20.3 & $\vdots$ & 1550 & 97.6 & 60.6 & $\vdots$ \\
\hline 20 & $i$ & 174.5 & $i$ & 1596 & 5.6 & 409 & $\vdots$ \\
\hline 20 & $i$ & 1204 & $\dot{1}$ & $155 \times 6$ & $47 \div: 1$ & 206.1 & $i$ \\
\hline 15 & $i$ & 78.1 & $i$ & $15 \cdot 6$ & $4 \therefore \therefore$ & 160.6 & $\vdots$ \\
\hline 10 & i & 46.1 & $:$ & $156 \cdot 6$ & 41.6 & 95 & 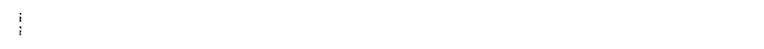 \\
\hline 5 & i & 25.1 & i & 156.6 & 41.4 & 51.9 & $\vdots$ \\
\hline 0 & 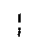 & 127 & $i$ & $156 \%$ & 41.1 & 26.1 & : \\
\hline-5 & $i$ & 6.2 & $\vdots$ & 1550 & 40 & 12.7 & : \\
\hline$-\cdots$ & $:$ & 4.5 & : & 155.6 & 40.6 & $9: 4$ & : \\
\hline Tota & 1 & 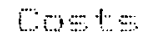 & & & & 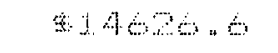 & $i$ \\
\hline 90 & $\mathrm{i}$ & 14.6 & ! & 1019. & 5.4 & $4 \pm \ldots$ & $\vdots$ \\
\hline 85 & $\vdots$ & 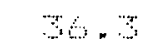 & i & 1007.9 & 50 & 91,9 & $i$ \\
\hline 90 & $:$ & 99. & $\vdots$ & $194 \%$ & $4: \cdots$ & 29, & 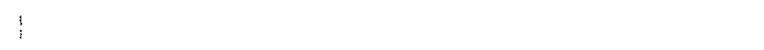 \\
\hline 78 & 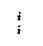 & 216 & 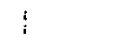 & अष् & $\% \theta \cdot \bar{c}$ & 455 & i \\
\hline 70 & $\vdots$ & 36.6 & : & $6 \theta_{n} 1$ & in & $5 / 6$ & : \\
\hline 65 & i & $46 \div$ & : & 59 & 27,2 & - 1,2 & 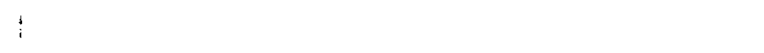 \\
\hline 60 & $i$ & $50 " 4$ & $i$ & 57.5 & $24: 4$ & $610 \times$ & $\vdots$ \\
\hline 56 & $i$ & $49 \%$ & $\vdots$ & 927.5 & 21.6 & 626 a & $i$ \\
\hline 50 & $!$ & $44 \% .6$ & $\vdots$ & 37 & $\because$ & 74.0 & : \\
\hline $4:$ & $!$ & $42=7$ & 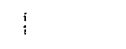 & 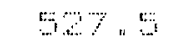 & 3.9 & 67.9 & $\vdots$ \\
\hline 40 & $!$ & $41 \Xi 7$ & $i$ & 527.5 & 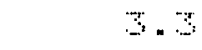 & 60.4 & $i$ \\
\hline 25 & $\vdots$ & 417.0 & $\dot{1}$ & 527,5 & $y_{n}$ & 69.0 & i \\
\hline 30 & ! & 417.4 & : & 527.5 & $3 \ldots$ & 69.0 & i \\
\hline 25 & $!$ & 395.2 & $i$ & 527.5 & 3 & 65.4 & i \\
\hline 20 & $i$ & 36.9 & : & $527 \times 5$ & $\exists$ & 55.7 & $i$ \\
\hline 15 & $i$ & 252.8 & $i$ & 527.5 & $3 \times 3$ & 41.6 & ! \\
\hline 10 & i & 165.7 & i & 527.5 & 3,3 & 27.4 & $i$ \\
\hline 5 & $\vdots$ & 96.3 & $!$ & 527.5 & 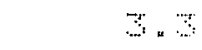 & 15.9 & $\vdots$ \\
\hline 0 & : & 50.9 & $i$ & 527.5 & $\exists$ & 8.4 & i \\
\hline-5 & i & 25.5 & : & 527.5 & 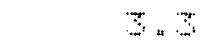 & 4.2 & $!$ \\
\hline-6 & ! & 21.1 & $\vdots$ & 57 & 5.5 & 3.5 & $i$ \\
\hline Tota & & $\cos t=$ & & & & अ68.6 & 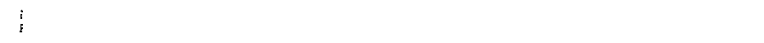 \\
\hline
\end{tabular}




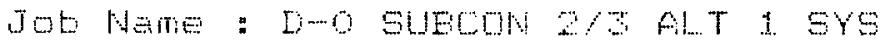

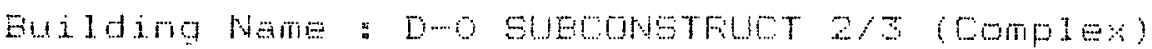

Eite Name " AUFOFA IL. FEFII, User DEtined
Date: $: 11-16-87$

Ger'i i Number. 601178620

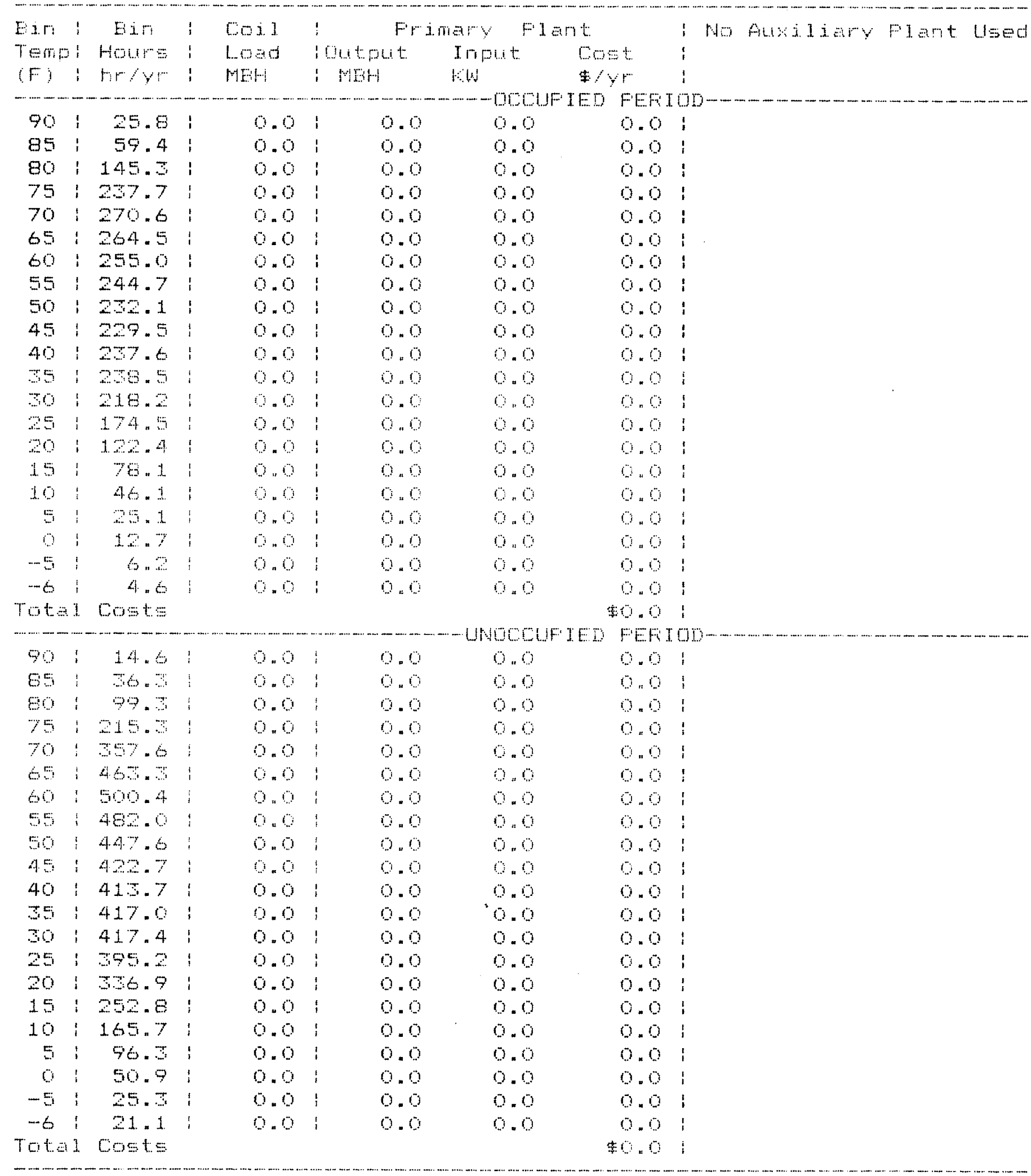




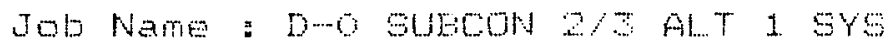

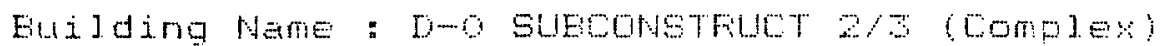

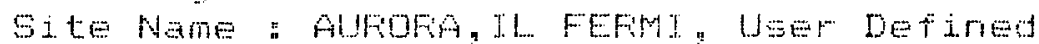

Date : $11-16-67$

Eerial Number:

60117862.0

\begin{tabular}{|c|c|c|c|c|c|c|c|c|c|c|c|}
\hline Ein & $\vdots$ & Ein & 1 & Fer imeter & Fans & $\dot{1}$ & Inter $=$ iop & $\mathrm{Fan}=$ & $i$ & $A 11$ & Fumps \\
\hline Temp & $i$ & Houre & $i$ & Iriput & $C \omega=t$ & $i$ & Input & {$[0 \equiv t$} & $i$ & Input & $\operatorname{Cos} t$ \\
\hline$(F)$ & $i$ & $\mathrm{mr} / \mathrm{yr}$ & $i$ & $\mid x$ & $\approx / y r$ & $!$ & $F W$ & $+y r$ & i & 16 & $\$ / Y r^{\prime}$ \\
\hline 90 & $!$ & 25.8 & 1 & $2 \because 4$ & 30.2 & $i$ & 0.1 & 0.2 & ! & 21.7 & 28.2 \\
\hline 85 & $\vdots$ & 59.4 & ; & $2+4$ & 69.6 & 1 & 0.1 & 0.4 & $i$ & 21.9 & 65.0 \\
\hline 80 & i & 145.3 & $i$ & 23.4 & 170.2 & 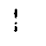 & 0.1 & 1.0 & $!$ & 21.9 & 159.0 \\
\hline 70 & $!$ & 270.6 & $i$ & 23.4 & $\because 17.0$ & $!$ & 0.1 & 1.8 & $i$ & 21.9 & 296.2 \\
\hline 65 & $i$ & 264.5 & $:$ & 25.4 & 309.8 & 1 & 0.1 & 1.8 & $i$ & 21.9 & 289.5 \\
\hline 60 & $!$ & 255.0 & $i$ & $2 \Xi .4$ & 296.7 & $i$ & 0.1 & 1.7 & 1 & 21.9 & 279.1 \\
\hline 5.5 & 1 & 244.7 & $i$ & $2 x, 4$ & 206.6 & $i$ & 0.1 & 1.6 & $i$ & 21.9 & 267.8 \\
\hline 50 & $!$ & $252-1$ & $i$ & $2=4$ & 271.9 & 1 & 0.1 & 1.5 & $i$ & 21.9 & 254.0 \\
\hline 45 & 1 & 229.5 & $i$ & $23-4$ & 268.8 & 1 & 0.1 & 1.5 & $i$ & 21.9 & 251.2 \\
\hline 25 & $i$ & 174.5 & $\vdots$ & $27, A$ & $204 \times 4$ & $\vdots$ & 0.1 & 1.2 & $?$ & 91.9 & 191.0 \\
\hline 20 & 1 & 122.4 & : & $2 y-4$ & 143.4 & $\vdots$ & 0.1 & 0.5 & $i$ & 21.9 & 154.0 \\
\hline 15 & 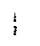 & 78.1 & $i$ & 27,4 & 91.5 & $\vdots$ & 0.1 & 0.5 & $i$ & 21.9 & 85.5 \\
\hline 10 & 1 & 46,1 & $i$ & $3 \cdot 4$ & 540 & $i$ & 0.1 & $y=\because$ & $i$ & 21.9 & 50.5 \\
\hline 5 & $!$ & 25.1 & $i$ & 20.4 & 29.4 & $\vdots$ & 0.1 & 0.2 & $i$ & 21.9 & 27.5 \\
\hline 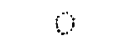 & $i$ & $12=7$ & i & 29.4 & 14.7 & $i$ & 0.1 & 0.1 & 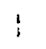 & 21.9 & 13.9 \\
\hline-5 & 1 & 6.2 & $\vdots$ & $2 x_{4}$ & 7. & $\vdots$ & $\theta=1$ & 9.0 & 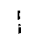 & 21.9 & 6.8 \\
\hline-6 & $i$ & 4.6 & $i$ & 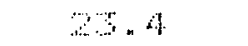 & 9.4 & $\vdots$ & 0.1 & 0.0 & $\vdots$ & 21.9 & 5.0 \\
\hline Toten & 1 & $C D \equiv t=$ & & & $\$ 964.6$ & $\vdots$ & & $\$ 2.8$ & $i$ & & +5424.1 \\
\hline 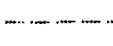 & & - & 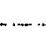 & & 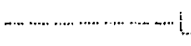 & 140 & GUF]ED FEF & $\because I O D \cdots$ & & - & $-(---1-1-$ \\
\hline 58 & 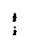 & 482.0 & $i$ & 28,4 & 564.6 & $\vdots$ & i. 1 & $\because 2$ & $i$ & 21.9 & 527.5 \\
\hline 50 & $i$ & 447.6 & 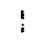 & $2 y=4$ & $94, y$ & $i$ & $i=1$ & $\because .0$ & 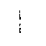 & 21.9 & $48 \% .9$ \\
\hline 45 & $i$ & 42077 & $\dot{i}$ & 20,3 & 49,1 & $\vdots$ & 1.1 & $\therefore \cdot 9$ & $\vdots$ & 21.9 & 462.6 \\
\hline $4 \%$ & $i$ & $41 . .7$ & 1 & $2 \div 4$ & 484.6 & $i$ & 0.1 & $\therefore 8$ & $i$ & 21.5 & 452.8 \\
\hline $3 \mathrm{i}$ & $i$ & 417.0 & $i$ & 23,4 & 486.5 & $\vdots$ & 9.1 & $\therefore .8$ & $i$ & 21.9 & $45:-4$ \\
\hline$\therefore 0$ & 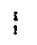 & 417.4 & $i$ & 28,4 & 483.9 & $\dot{s}$ & 0.1 & 208 & $i$ & 21.9 & 45.6 \\
\hline 25 & 1 & 395.2 & $i$ & 234 & 462.9 & $i$ & 0.1 & 2.6 & $i$ & 21.9 & 4.2 .5 \\
\hline 20 & 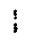 & 76.9 & $i$ & 27,4 & 794.6 & $i$ & 0.1 & $\because 2$ & i & 21.9 & $\$ 68.7$ \\
\hline 15 & 1 & 252.8 & $i$ & 23,4 & 296.1 & 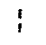 & 0.1 & 1.7 & $!$ & 21.9 & 276.7 \\
\hline 10 & 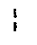 & 165.7 & $i$ & $2=4$ & 194.1 & 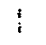 & 0.1 & 1.1 & $i$ & 21.9 & 181.4 \\
\hline 5 & 1 & 96.3 & : & 20.4 & $112 \times 9$ & $i$ & 0.1 & 6.6 & $!$ & 21.9 & 105,4 \\
\hline 0 & 1 & 50.9 & $i$ & $\therefore=4$ & $59 \times 6$ & $!$ & 0.1 & $\theta, \bar{z}$ & $\mathbf{i}$ & 21.9 & 55.7 \\
\hline-5 & $i$ & 25.7 & : & 25,4 & 29.8 & 1 & 0,1 & 0,2 & $\frac{1}{3}$ & 21.9 & 27,7 \\
\hline-6 & $i$ & 21.1 & 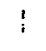 & 23.4 & $24: 7$ & $\vdots$ & i. 1 & 0.1 & i & 21.9 & 23.1 \\
\hline Tota & & $\omega=t=$ & & & क्ष & $\dot{3}$ & & 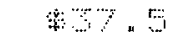 & $i$ & & $\$ 6160,5$ \\
\hline
\end{tabular}




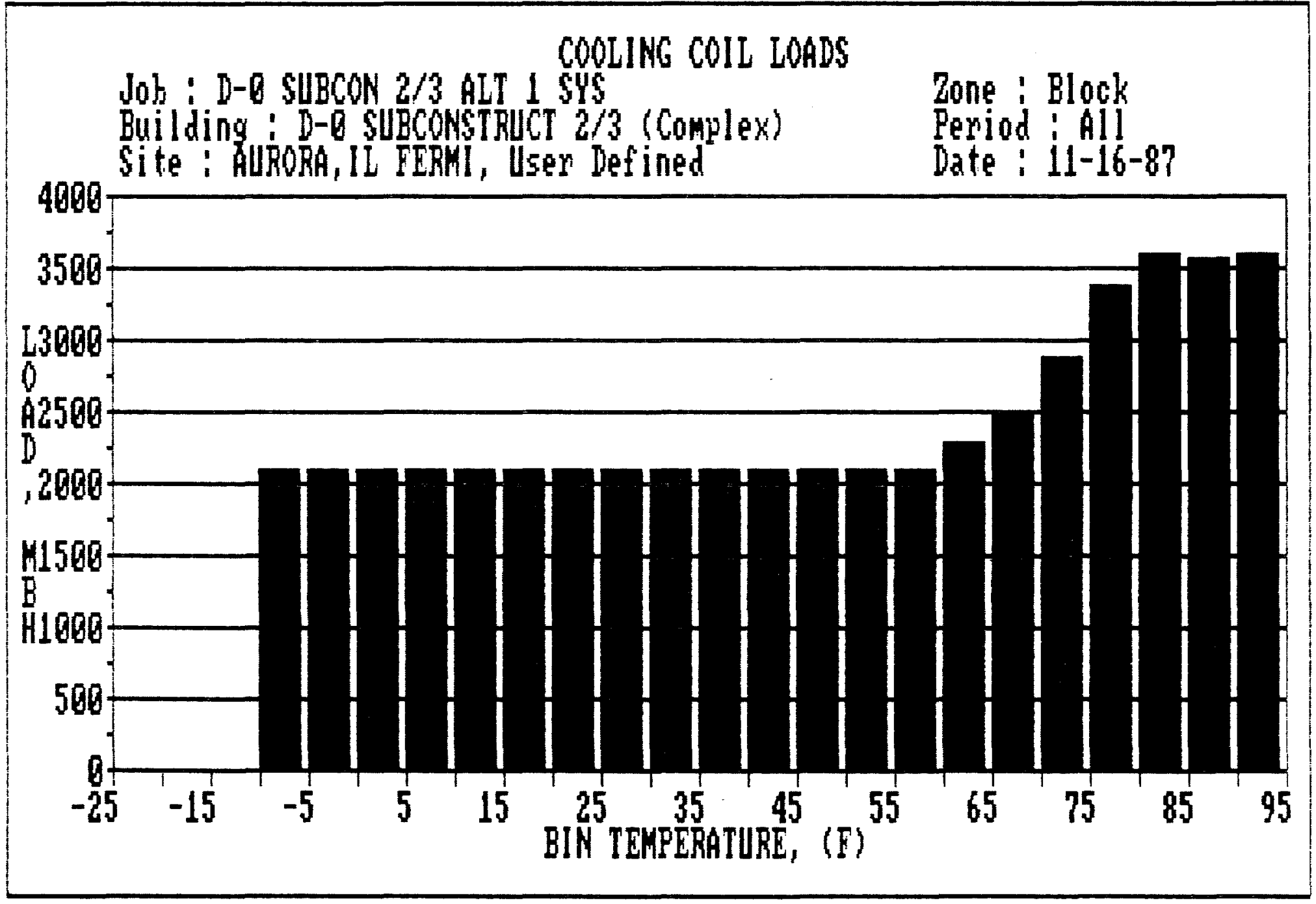




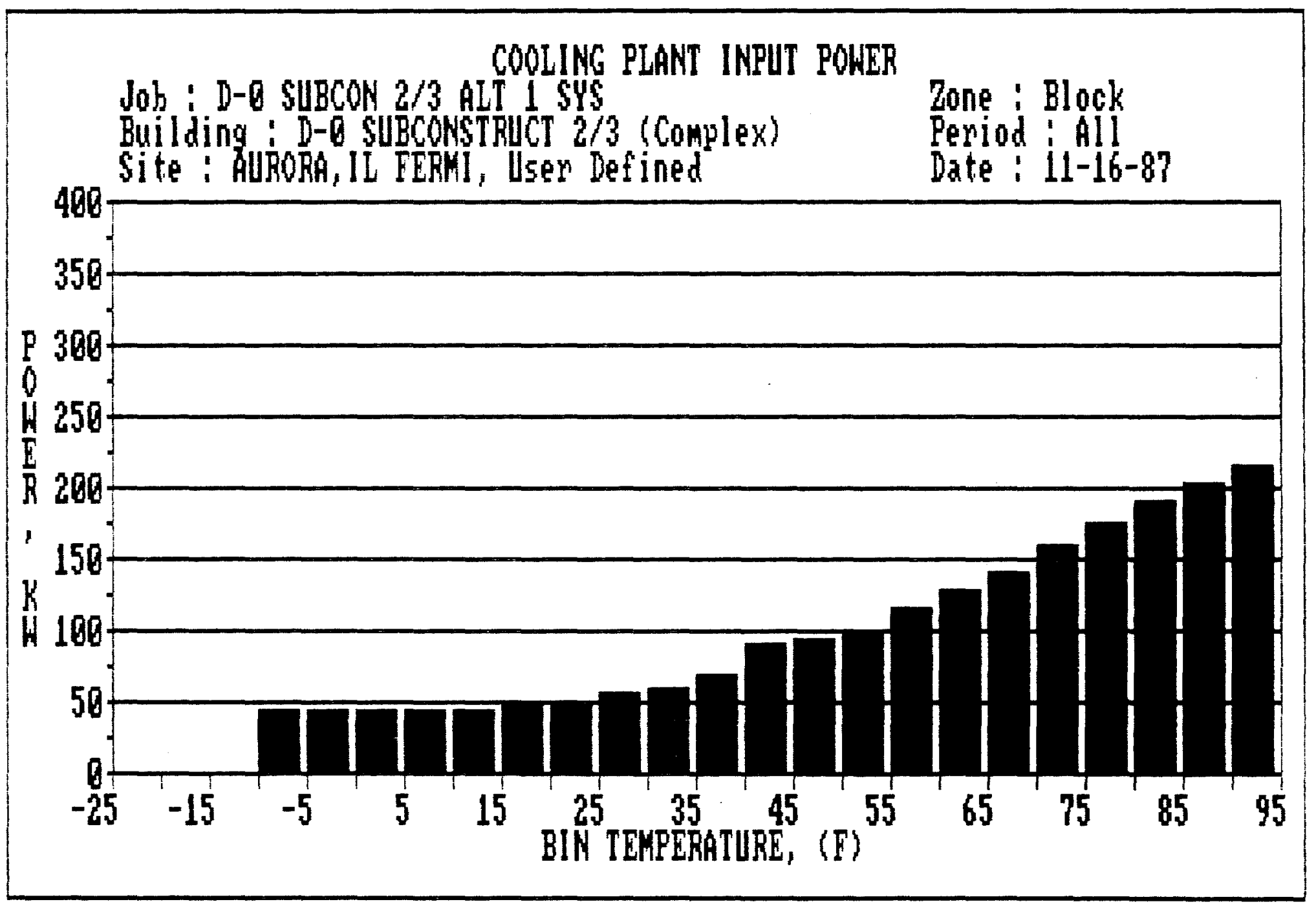




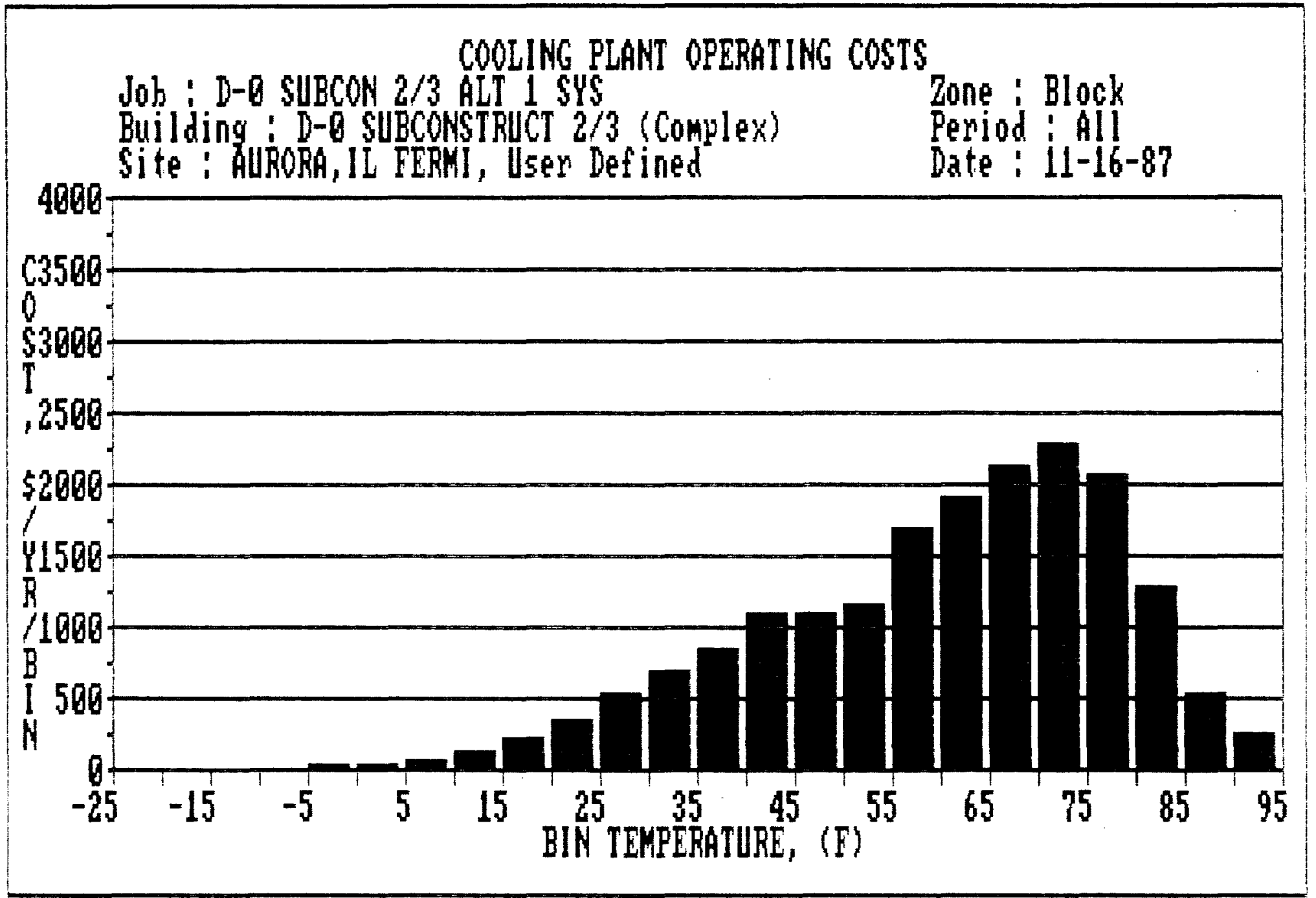




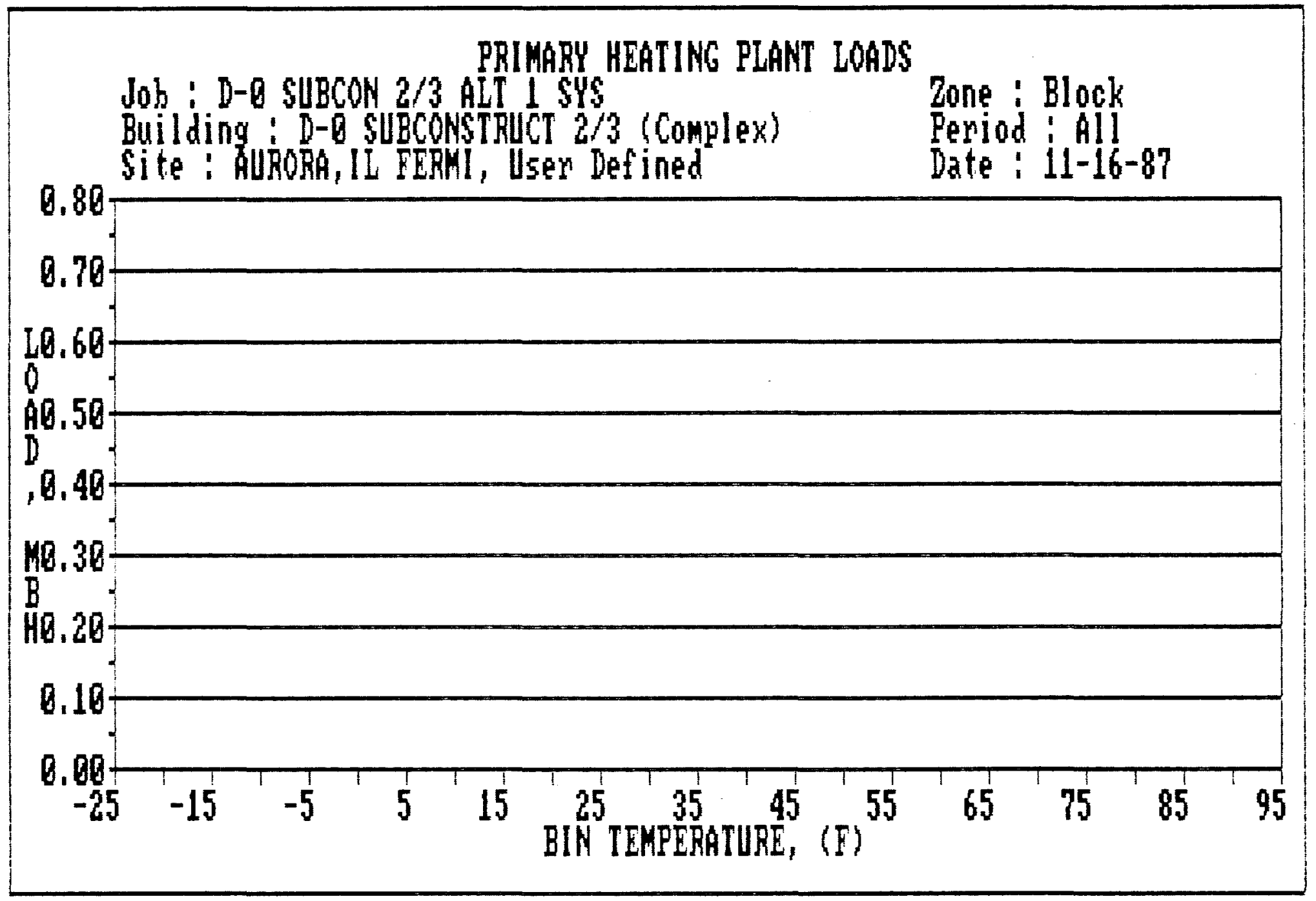


FAN OPERATING COSTS

Joh : D-O SUBCON $2 / 3$ ALT 1 SYS

Building : D-0 sUBCONSTRUCT $2 / 3$ (Complex)

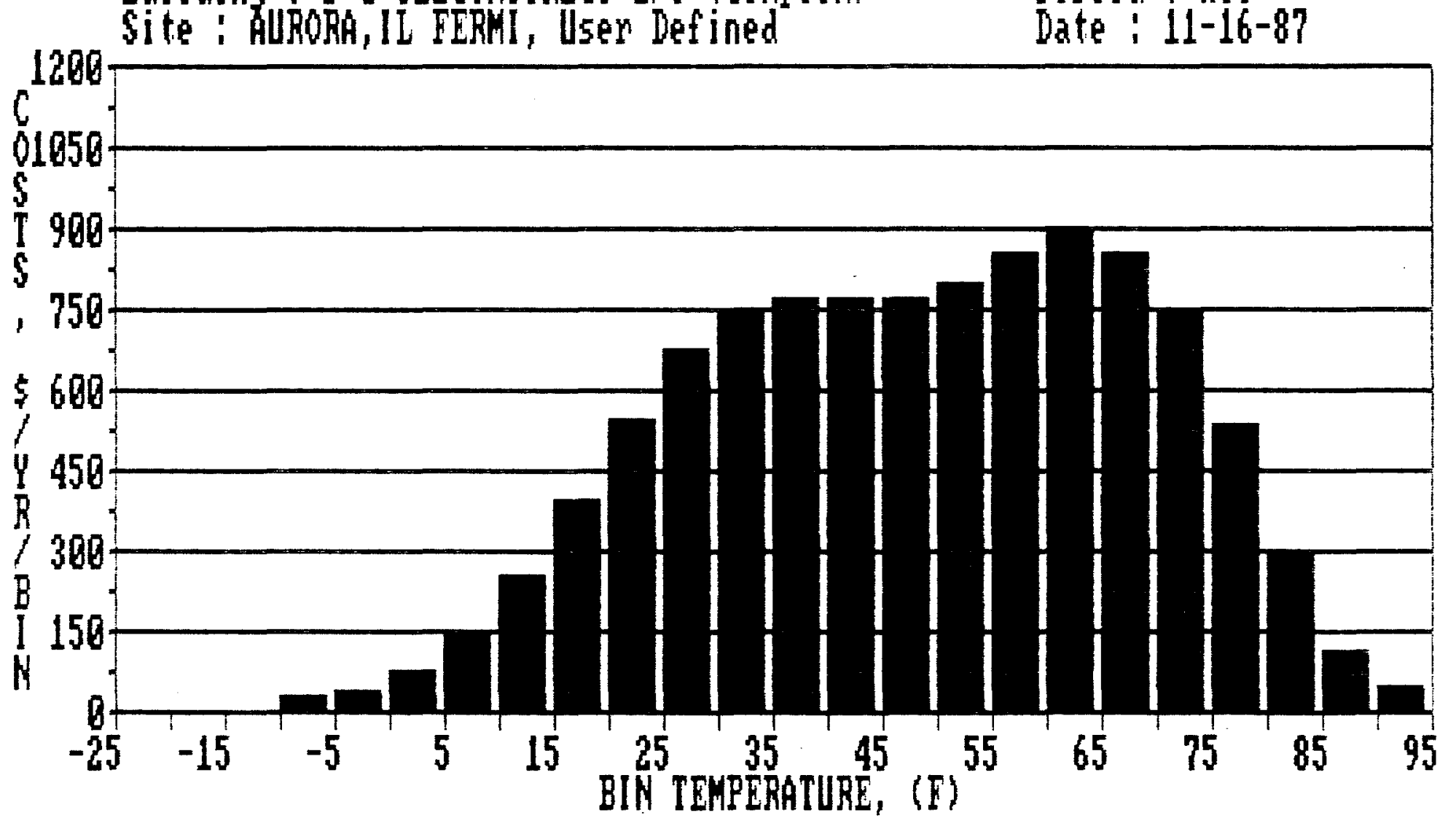

Zonẹ : Block

Period All

Date: 11-16-87 


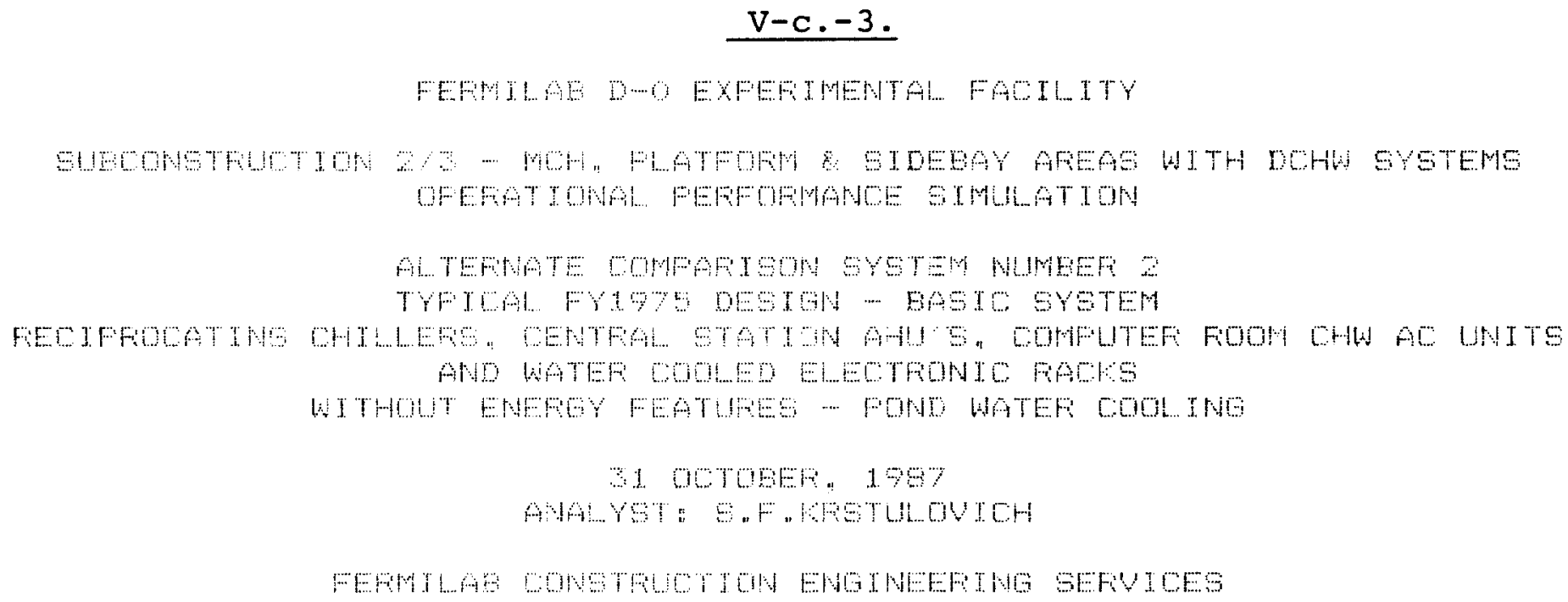


Job Nane : D-0 SUPOON 2/3 FY1975 5 Y

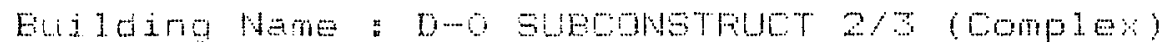

Fage 1

site : AUFCFA, IL FEMM: USEF Defined

Date : $11-16-67$

60117962.0

seope of Analysis : Cooldio and Heatimo Systems

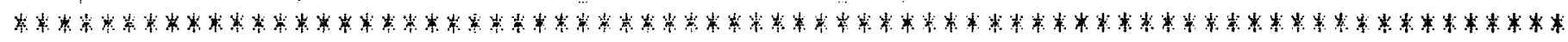

A. AF HANDING SYSTEM

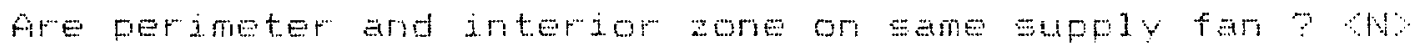

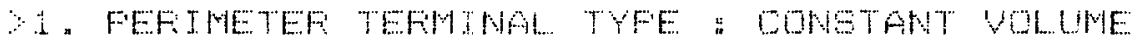

Supply fan type

Supply fan total static pressure $=2.50$ in. wa.

Fieturn tan type: Controlled pitoh axial

Fieturn fan totel. static pressure = $=1.25$ in. 49 .

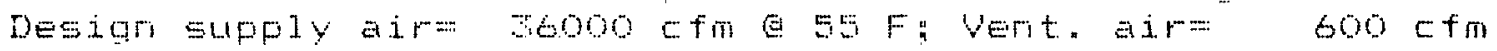

Are cooling terminal = used for heating ? ry

An economizer is not lEEd.

Is a ventilation reclaim device used $?$ lo

Are vertilation air dampers wosed for unoceupied periods

Z. INTEFIGF: TEFMTNAL TYFE: CONSTANT VOLUME

Supply tri type : Frmare curved

supoly fan total stato pressume o. ol im. wo.

A return tar d

hos

Am; eromizer i

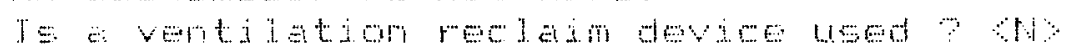

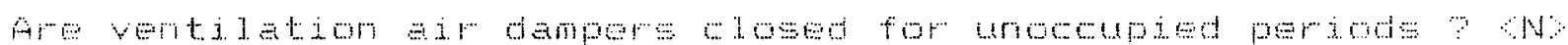

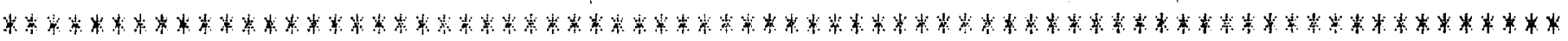

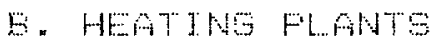

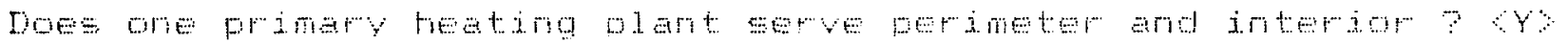

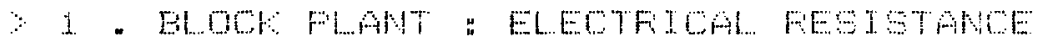

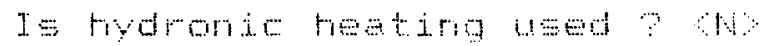

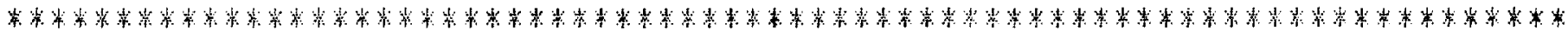




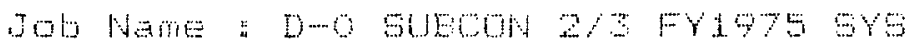

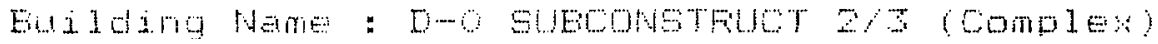

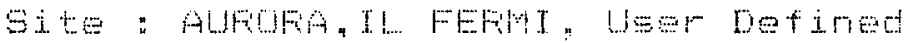

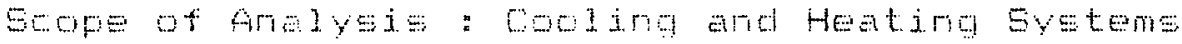

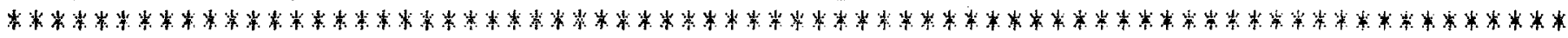

[. COLLING FLARTS

DoEs ore plat serve perimeter and interior ? ry

1. BLOCF FLANT: FECIFFUCATING WATEF: COOLED

Capacity a $85 \mathrm{~F}$ entering water $=230.0$ tons

fWiTon e $85 \mathrm{~F}$ entering water $=1.00 \mathrm{kw}$ ton

I hydronie cooling uझed ? YY

Is chilled water reset used? CN

Is hot gas bypase used ? N

Is condenser performance altitude adjusted ? $N$

Is there one compressor per condenser circuit $\because 4 y$

Are compressors cycied $?$ Wh

Heat sint: type : Closed circuit cooling tower

Minimum entering water tempereture $=40 F$

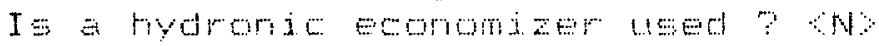

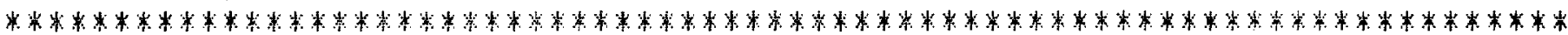

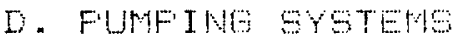

\begin{tabular}{|c|c|c|c|c|c|c|c|c|c|}
\hline$i$ & & : & & 19 & ETEP & i & & EATOF & \\
\hline 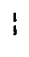 & Sy=ten & 1 & at. & $\vdots$ & $d F,+t+40$ & $i$ & $d T, F$ & i dF' & $f t w g$ \\
\hline d & Etrid leot ander & $i$ & 10.0 & $!$ & 100.00 & $\vdots$ & 0.96 & $\vdots$ & 0.00 \\
\hline i & Hot wetser & $i$ & 0.00 & $i$ & 0.90 & $\vdots$ & 0.00 & 1 & 0.00 \\
\hline & 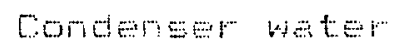 & $\vdots$ & 10.00 & $\vdots$ & 120,0 & 1 & 0.00 & $\vdots$ & 0.00 \\
\hline$f$ & Grouid water & $\vdots$ & 0.03 & : & 0.0 & $\vdots$ & 0.00 & $i$ & 9.00 \\
\hline
\end{tabular}

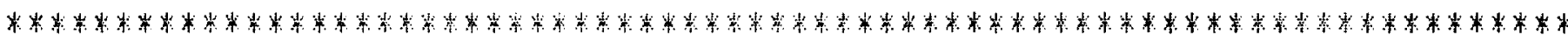

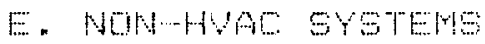

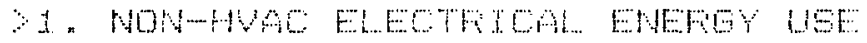

\begin{tabular}{|c|c|c|c|c|c|c|c|}
\hline antimg & $f w=$ & $00 \mathrm{cmi}=0$ & $=::$ & 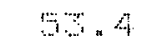 & $\because \forall:$ & Wmotelo ied & 48.15 \\
\hline $1 \div 4$ & $:$ & a- apd & $=::$ & $660 \%$ & Lw: & Urom anjed & 200 \\
\hline$\because \cdots$ & $:$ & 0000104 & $\because \cdots$ & 0 & $6 \ln _{4}^{\pi}$ & Urocou 100 & $0 \times$ \\
\hline
\end{tabular}

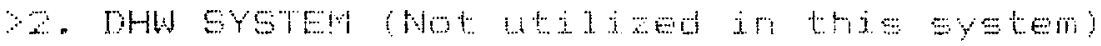

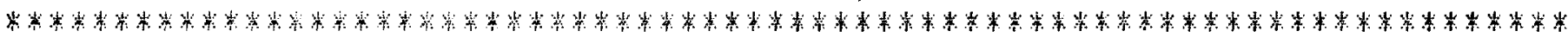

FIEL COIG:

\begin{tabular}{|c|c|c|c|c|c|c|c|c|c|}
\hline$\because .1$. & 1 & \multicolumn{3}{|c|}{ 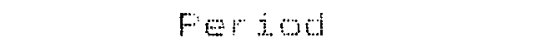 } & $i$ & 2 . OTHEF FUELB & $i$ & Uit & $\cos$ \\
\hline ELECTFICAL & $i$ & Decupied & $i$ & Urioculpied & $\vdots$ & - & & $-\cdots-$ & -- \\
\hline ENEFIGY USE & $i$ & 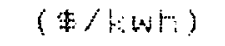 & $:$ & $($ t $/(a, H)$ & $i$ & : Natura $=1$ & i & $\mathrm{n} / \mathrm{a}$ & Q \\
\hline & - & 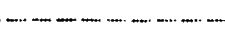 & & 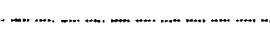 & .... & :FLIEI OII & & $n / a$ & $4 / 4.5 .901$ \\
\hline Compresesor & $\vdots$ & 0.050 & $i$ & 0.050 & $i$ & :Fropane & & $\pi / a$ & $\$ / 1 \mathrm{~b}$ \\
\hline Fiesistance & ; & 0.050 & 3 & 0.050 & $i$ & i Femote Heating & 1 & $\Pi / a$ & \$/METU \\
\hline Iriductive & i & 000 & $i$ & 0.050 & 1 & SRemote Coolimg & $i$ & $n / a$ & \$ $/$ METU \\
\hline
\end{tabular}




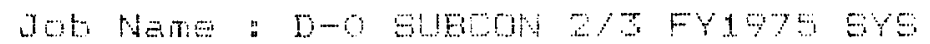

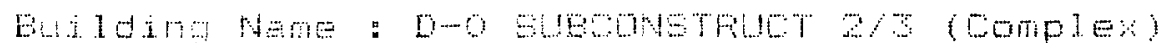

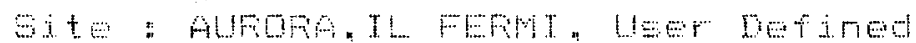

$6=10: 11-16-97$

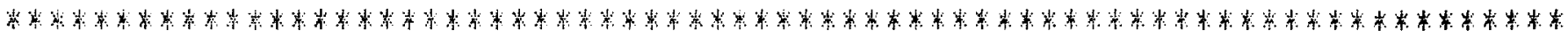

HVAC Total
HeO ENGG

componem

Elestion (OCE)

Electric (LMoce)

Natural Gis

Fle 1 Oi. 1 .

Fropene

Fiemote Heating

Fiemote Cooling

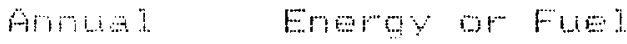 \\ Eost Umite}

(tor)

mor $=$ mino

\begin{tabular}{|c|c|c|}
\hline 32727 & 6545.1 & fowh / yr \\
\hline 3065 & 601502 & fowh $/$ yer \\
\hline 0 & 0 & therMS/Yr \\
\hline i & $\mathrm{o}$ & 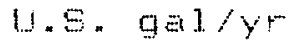 \\
\hline 0 & 0 & $I b / y^{r}$ \\
\hline 0 & 0 & METLYYF \\
\hline 0 & 0 & METU/Yr \\
\hline
\end{tabular}

627

MON-HYSE ENERG

\begin{tabular}{|c|c|}
\hline$E$ Aecto ic & $(0) \ldots)$ \\
\hline$E \perp e c t r i c$ & (1) Lroos) \\
\hline$-1 .-1-1-1$ & 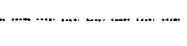 \\
\hline Nom-HAl & $=1$ \\
\hline
\end{tabular}

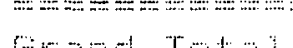

Trtetion

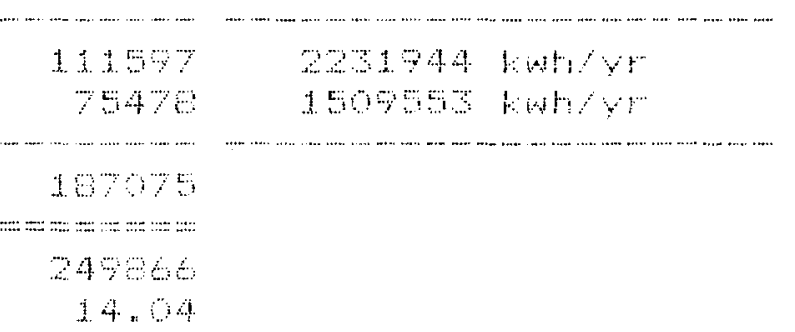

\begin{tabular}{rr} 
DOE FUF & DOE FIF \\
$(1000$ ETU) & $(1000$ BTU \\
\hline 7592563 & 2253915 \\
6575101 & 2052243 \\
0 & 0 \\
0 & 0 \\
0 & 0 \\
0 & 0 \\
0 & 0 \\
-14567664 & 4286150
\end{tabular}

\begin{tabular}{|c|c|}
\hline 25890545 & 761762 \\
\hline 1750014 & 9152104 \\
\hline 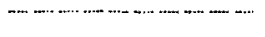 & - \\
\hline 490159 & 1276920 \\
\hline 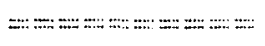 & 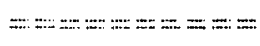 \\
\hline 579692 & 1705806 \\
\hline 96,69 & 950 \\
\hline
\end{tabular}

Hes Sumbirin:

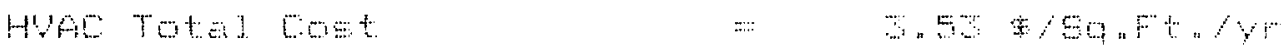

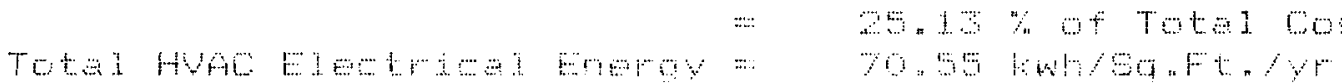

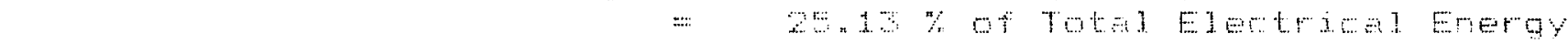

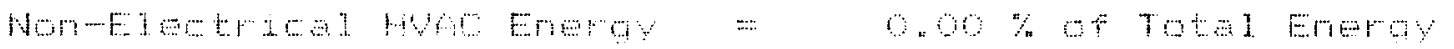

$10 y=$

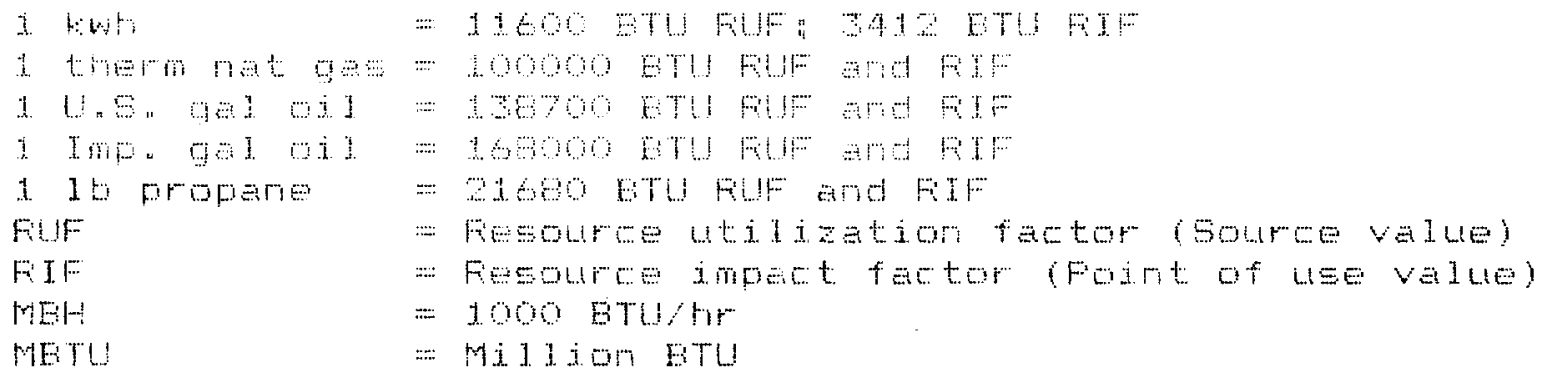




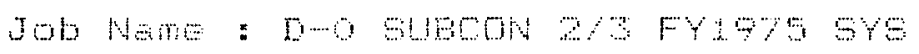

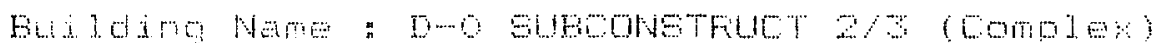

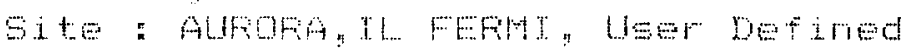

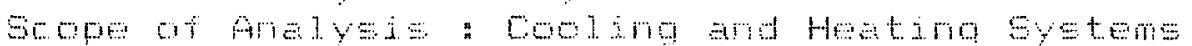

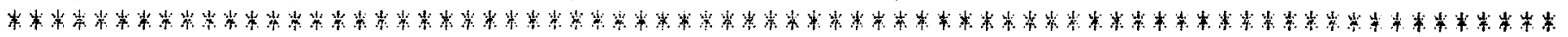

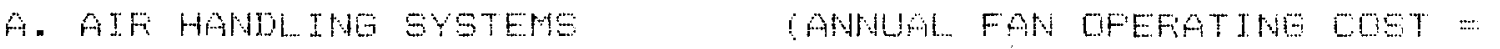

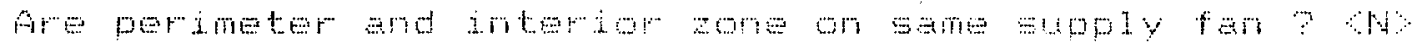

$\$ 12.865$

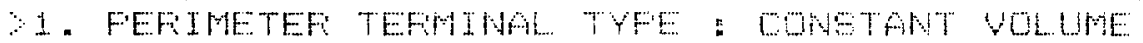

Supply fan type : Formatod aured

Supfly fan total static piessure $=2.50$ in. wg.

Feturn fan type: Comtrolled piton arial

Feturn fan total static pressure $=1.25$ in. wa.

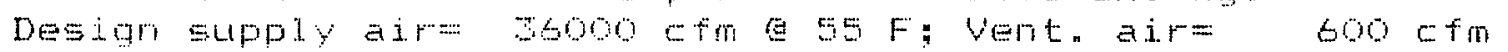

Ate cooling terminal

An economizer is riot used.

$I \subseteq$ a ventilation reclaim device used ? NN

Are wentilation atr dampers olosed for unocoupied periods 7 a

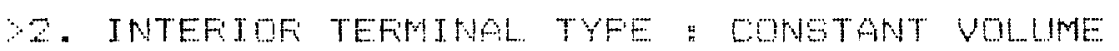

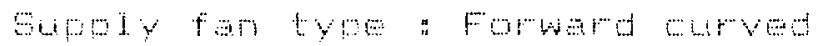

gupj

A

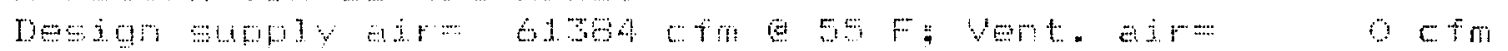

An wom

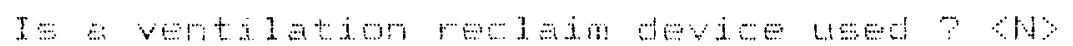

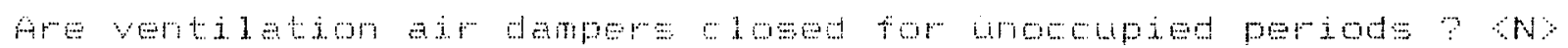

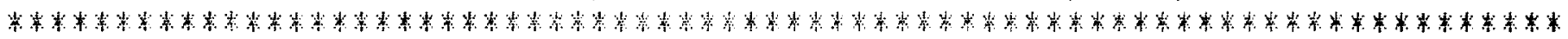

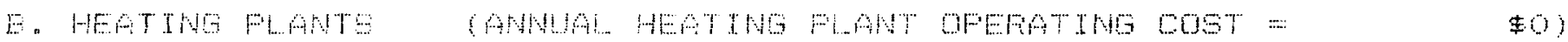

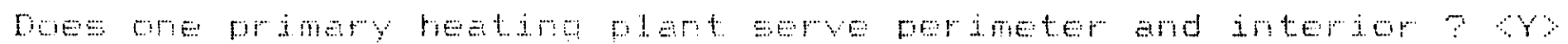

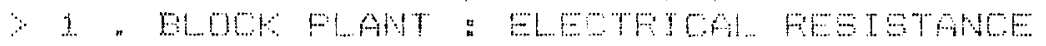

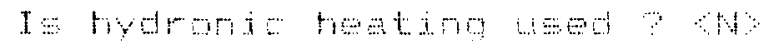

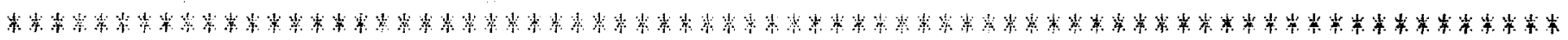




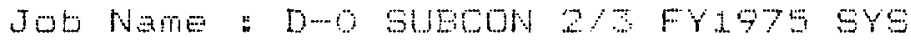

Euilding Name : D-O SUECONSTFUCT 2/3 (COMplex)

$F=2$

Site : AUFOFÁ, IL FEFHI, UEE DEfiried

Dett: $: 11-16-87$

क1. 17860

Sowp of Analysis: Cooling and Heating systems

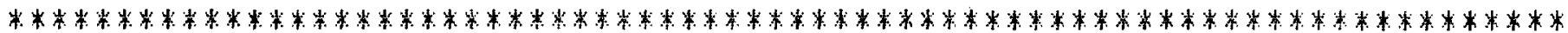

C. COOLING FLANTS SANHAL COOLING FLANT DFEFATING COST

$\$ 6,650)$

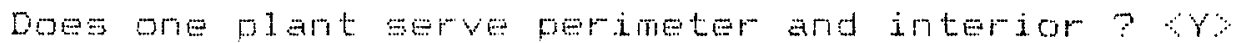

1. BLOCF FLANT " FECIFFOCATING WATEF COOLED

Capacity $\mathrm{C} 8 \mathrm{~F}$ entering water = 230.0 tons

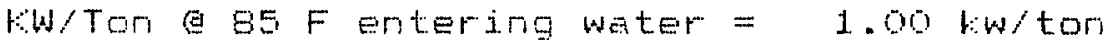

Is hydronic cooling used $?$ YY

Is chilled water reset Lsed $\because$ ru

Is hot gas bypass used 7 W

Is condenser performance altitude adjusted $?$ ov

Is there one compressor per condenser circuit $?$ $Y$

Are compressors cycied ? No

Heat sinf: type : C10sed cimcuit cooling tower

Minimum entering water temperature $=40 \mathrm{~F}$

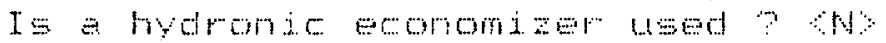

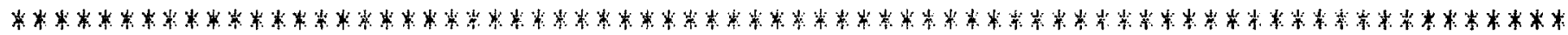

D. FUMFTHG SYSTEMS CANMUA FUMF OFEFATING COST=

$\$ 1,25 \%$

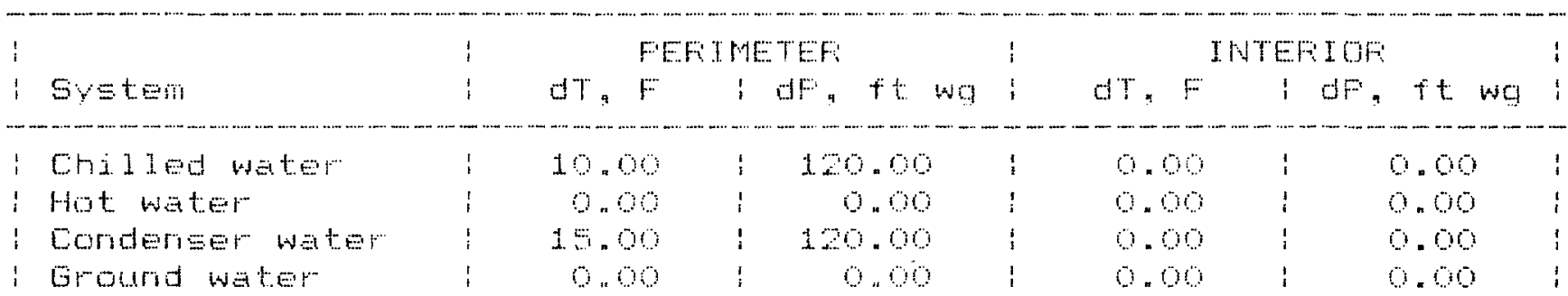

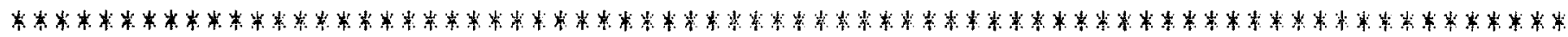

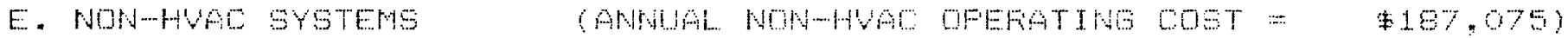

1. NOH-HVAL ELELTFITAL ENEFEY UEE

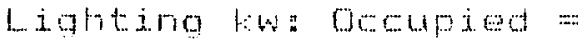

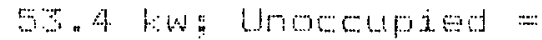
$49.1+\omega$
Misco.

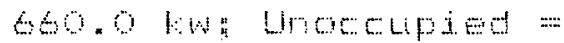
220.0 tw
Other low " acoupied
0.6 tw: Mocempied =
$0.01 \%$

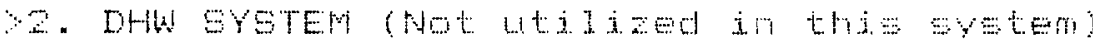

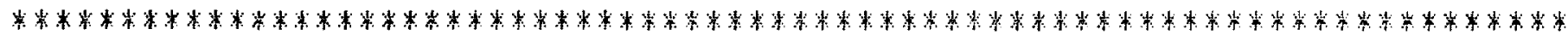

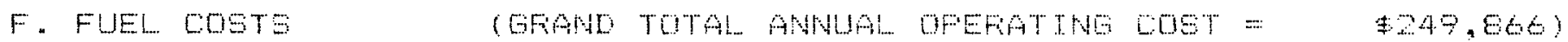

\begin{tabular}{|c|c|c|c|c|c|c|c|c|}
\hline 1 & $i$ & \multicolumn{2}{|c|}{ Feriod } & $i$ & 2 口 OTHEF FUELS & : & Unit & Cost \\
\hline ELECTFICAL. & 1 & OcEupied & InOCELPiEd & $i$ & $----n-1-1$ & & $--\ldots-n$ & $----\cdots$ \\
\hline ENEFEY LISE & $!$ & $(\$ / F(k)$ & (q) $\{$ : wh $)$ & $i$ & ivatural Gas & 1 & nila & oitherm \\
\hline 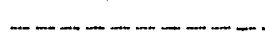 & - . & & 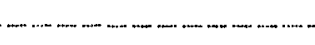 & $\ldots$ & FleIEI 011 & $i$ & nia & \$U.S. \\
\hline Compressur & $i$ & 0.050 & 0.050 & $i$ & ifroparie & 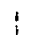 & $n / a$ & $\$ / 1$ t \\
\hline 7esistance & $i$ & 0.050 & 0.050 & , & iFenote Heating & $i$ & $n / a$ & D/METU \\
\hline Iriductive & $i$ & 0.050 & 0.050 & $!$ & Fiemote: Cooling & 1 & $n / a$ & D/METU \\
\hline
\end{tabular}


Jal

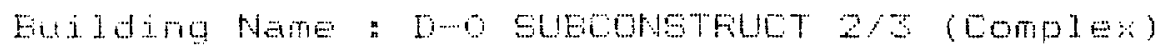
Site Name " AUFOFA, IL FEFMI, Woer Defined

$D a t e: 11-16-87$

Seriaj Numiber: $6117620 \%$

\begin{tabular}{|c|c|c|c|c|c|c|c|}
\hline Eiri & $\vdots$ & Sin & $i$ & 5.0 & $\operatorname{Cos} 1 \mathrm{ing}$ & FIarit & ! \\
\hline Temp & $!$ & Hotro & $\mathrm{CO} i$ & 1 Load & Input & $\mathrm{COO}=\mathrm{t}$ & 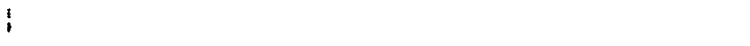 \\
\hline$(F)$ & $i$ & $\mathrm{hr} / \mathrm{yr}$ & $1 \quad 1$ & $M E H$ & $1+4$ & hy & $i$ \\
\hline$-\cdots-\cdots-\ldots$ & -... & $-\cdots-\cdots-\cdots$ & 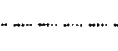 & $-(-2)-$ & 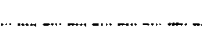 & OCQUFTE & FERIUI- $-\cdots$ \\
\hline 90 & i & 25.8 & i & 2584.0 & 216.8 & 279.71 & 1 \\
\hline 85 & 3 & 59.4 & $i$ & 2569.9 & 207.7 & 616.8 & ! \\
\hline 80 & $i$ & 145.7 & $i$ & 2550.1 & 19.9 & 1444.6 & $i$ \\
\hline 75 & $i$ & 27.7 & i & 2542.6 & 190.5 & 226.7 & ! \\
\hline 70 & i & 270.6 & $\vdots$ & 2529.5 & 102.5 & 2460.9 & $i$ \\
\hline 65 & $\vdots$ & 264.5 & : & 2516.7 & 174.9 & 23130 & $i$ \\
\hline 60 & $i$ & 255.0 & $i$ & 2504.2 & 167.7 & 21.9 .5 & i \\
\hline 55 & $i$ & 244.7 & $!$ & 2491.9 & $160 . \theta$ & 1966.9 & ! \\
\hline 50 & i & $2+2=1$ & : & 2480.0 & $15 \%, 1$ & 1. 741.4 & $\mathfrak{i}$ \\
\hline 45 & ! & 229.5 & $i$ & 2469.0 & 140.2 & 1608.3 & $!$ \\
\hline 40 & 1 & 237.6 & $!$ & 2450 . 1 & 130,9 & 1566.2 & $i$ \\
\hline 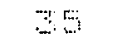 & $\vdots$ & 270,5 & $\vdots$ & 2447.1 & $120 \quad 2$ & 1456.9 & $i$ \\
\hline 30 & $i$ & 218.2 & $!$ & 24362 & 114.1 & 1244 & $i$ \\
\hline 25 & $i$ & 174,5 & $\vdots$ & 2425.2 & $10 \%=4$ & 920.6 & i \\
\hline 20 & $\vdots$ & $1 \cdots 44$ & $!$ & 2414.3 & 99.3 & 607.9 & 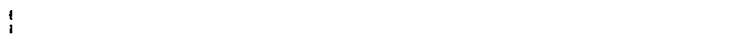 \\
\hline 15 & $:$ & 70.1 & $i$ & 2403.3 & 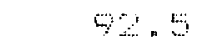 & $7+1,7$ & $i$ \\
\hline 10 & $\frac{1}{3}$ & $A 6,1$ & $i$ & $292 \times 4$ & 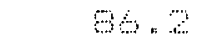 & 198.6 & 3 \\
\hline 5 & $i$ & 25.1 & $\vdots$ & 2891.4 & 96 & 101.2 & $i$ \\
\hline 3 & $i$ & 12,7 & $i$ & $2 \because 79$ & $6, z$ & 5.1 .0 & 3 \\
\hline-5 & $\mathrm{i}$ & $6: 2$ & 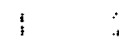 & 2859 & 79.9 & 24.8 & : \\
\hline-6 & $i$ & 4.6 & $\mathfrak{i}$ & 2867 & $7 \%$, & 18.4 & 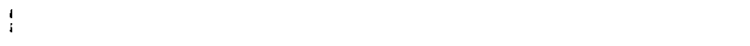 \\
\hline $\operatorname{Tot}:$ & 1 & $\cos =1=$ & & & & $32,39,0$ & : \\
\hline$-\cdots-\cdots$ & 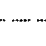 & 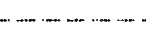 & $(\cdots+\cdots, \cdots \cdots$ & 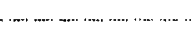 & 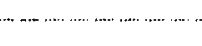 & -10000100 & 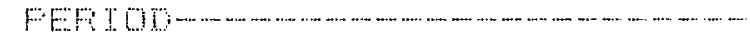 \\
\hline ? & : & 14.6 & 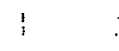 & 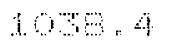 & +. & $5 \% .7$ & $\vdots$ \\
\hline 5 & $i$ & 5,3 & $i$ & 107,0 & 77.6 & 169,7 & $i$ \\
\hline$\theta$ & $i$ & 99,3 & 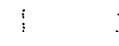 & $1+17+1$ & $7 x+4$ & 34,4 & $i$ \\
\hline $7 !$ & 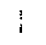 & $\because 5, y$ & $i$ & 100.4 & $6 \%, 4$ & 77.6 & $i$ \\
\hline 7 & $\vdots$ & $\because r$, & $:$ & 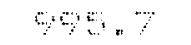 & $6: \cdots, 7$ & 11740 & $i$ \\
\hline 65 & i & $4,3,3$ & $:$ & 95,1 & $a+1$ & $1496=$ & $i$ \\
\hline 6 & 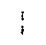 & 500.4 & $\vdots$ & 97.4 & 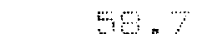 & $460, x$ & $\vdots$ \\
\hline 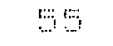 & 3 & $49 z$ & $i$ & 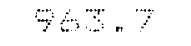 & 5,4 & $136+7$ & 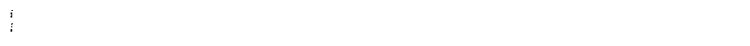 \\
\hline 5 & $\vdots$ & $447 \cdot 6$ & 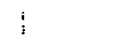 & 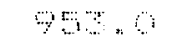 & 50.5 & 1199,6 & 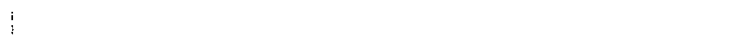 \\
\hline 45 & $\mathrm{i}$ & $42=7$ & $i$ & 94,6 & 49.9 & 969.9 & $\vdots$ \\
\hline 40 & $i$ & 41.7 & $:$ & 931.7 & 41.6 & 821.0 & $i$ \\
\hline 35 & $i$ & 417.0 & i & 921.6 & 77.6 & 704.3 & $i$ \\
\hline 30 & $i$ & 417.4 & $i$ & 910.3 & 3.9 & 707.3 & 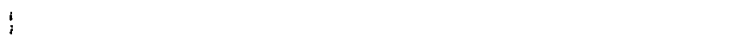 \\
\hline 25 & $i$ & 395.2 & : & 899.6 & 71.5 & 62.1 & $i$ \\
\hline 20 & 1 & 5.6 .9 & $i$ & $8 \theta 9.9$ & $31, \because$ & 59.5 & 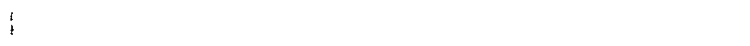 \\
\hline 15 & $i$ & 252.0 & 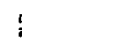 & 878. & अ० 8 & 589.7 & $\vdots$ \\
\hline 10 & : & 165,7 & $\vdots$ & 86.6 & का & 25.9 & 1 \\
\hline 5 & $i$ & 96. & 1 & 856.9 & $0=1$ & 145.0 & i \\
\hline 0 & $!$ & 50.9 & $\vdots$ & $846 \ldots$ & 29.6 & 75.8 & $\vdots$ \\
\hline-5 & 1 & $25, \cdots$ & $i$ & 85.6 & $2 \% .4$ & 27.2 & $\vdots$ \\
\hline-6 & $;$ & $21=1$ & 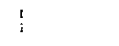 & बar. & क & $\because n$ & $\vdots$ \\
\hline$T 0 t=$ & & {$[\infty=+$} & & & & 28969 & $\vdots$ \\
\hline
\end{tabular}




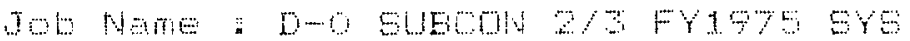

Date; $; 11-16-87$

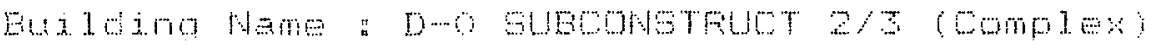

serial dumber:

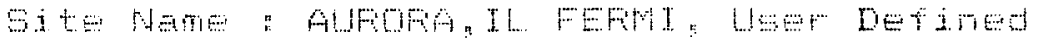

6011780.0

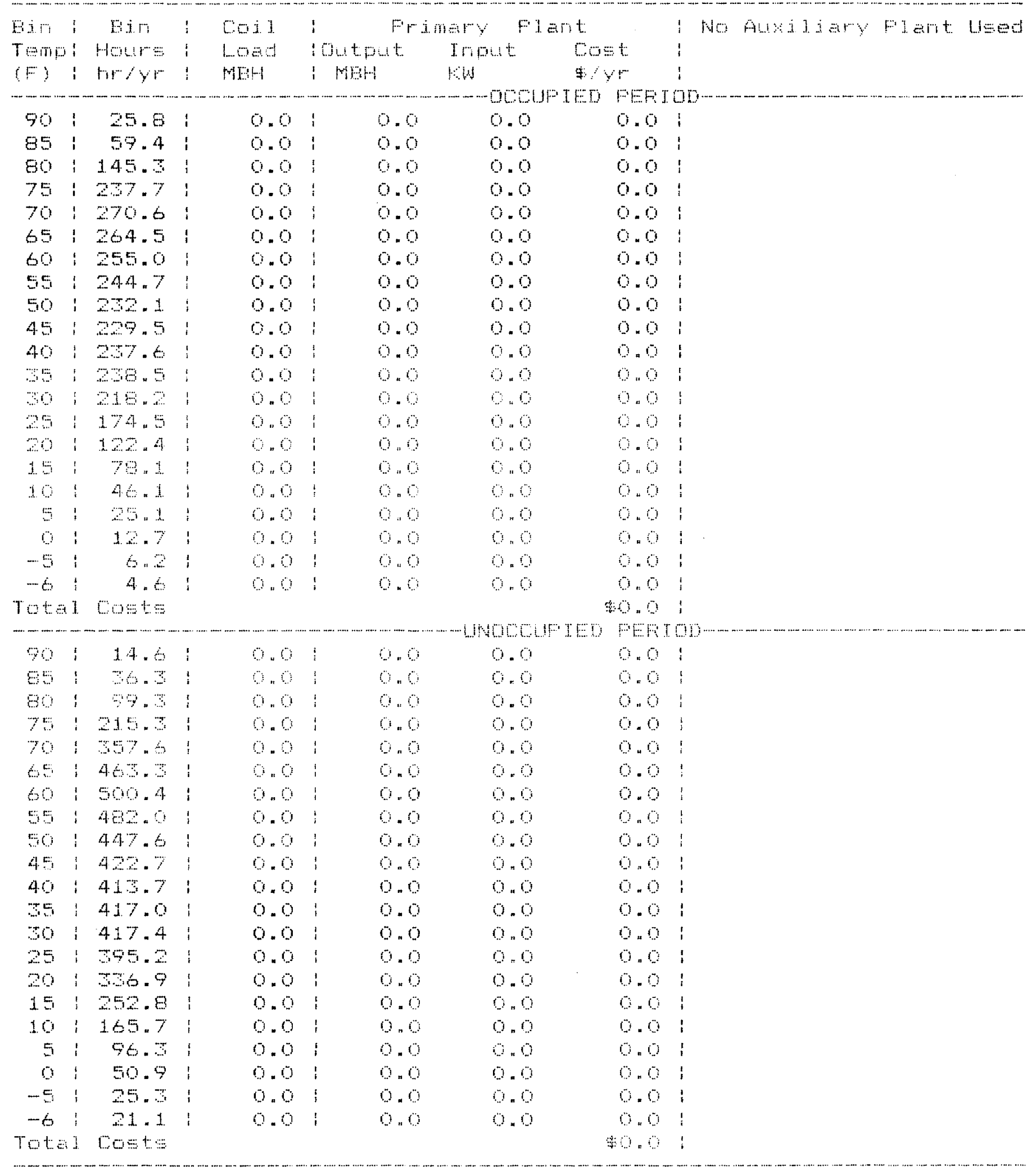


Date : $\quad 11-16-87$

SErig Nimber:

601178620

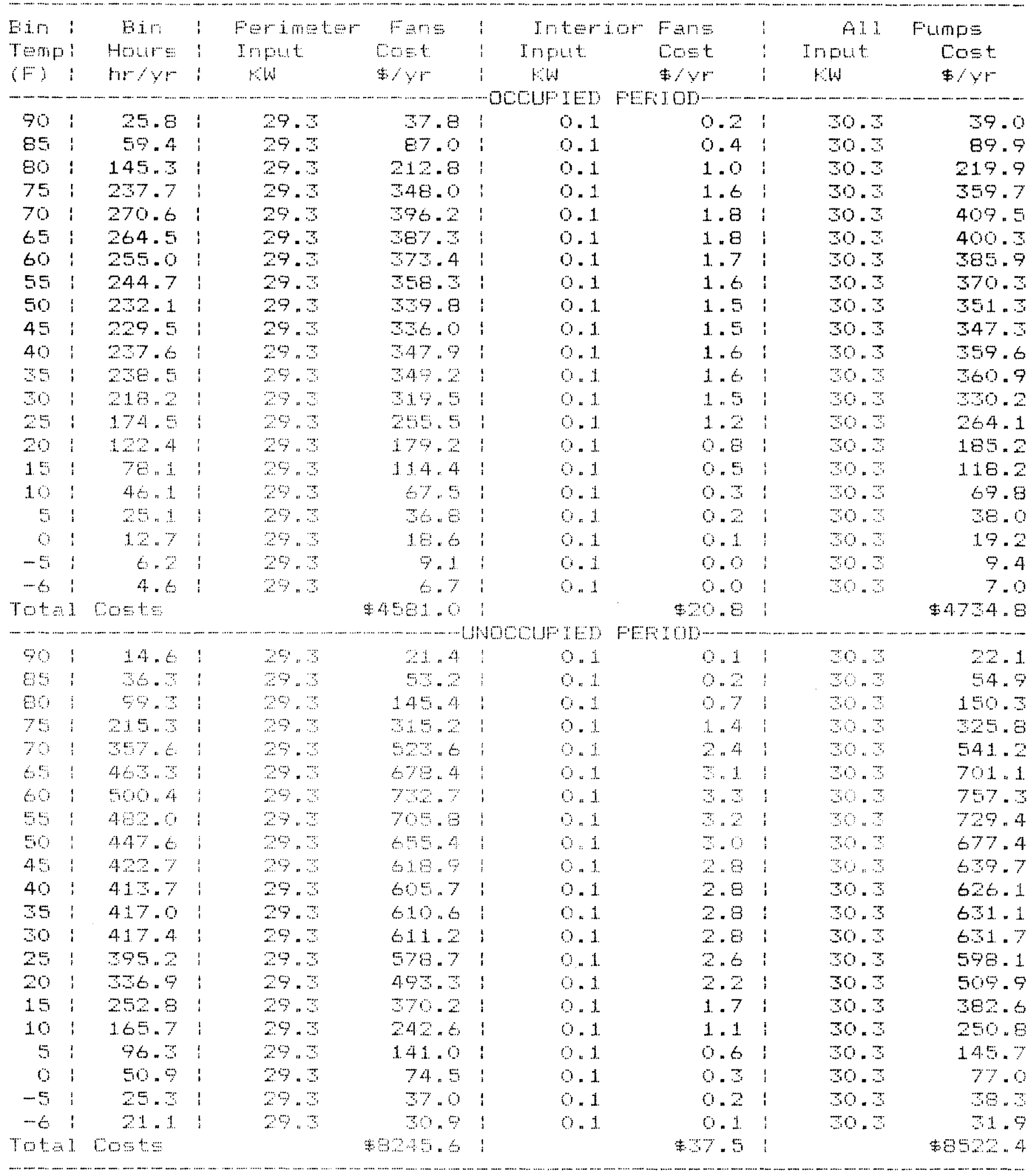




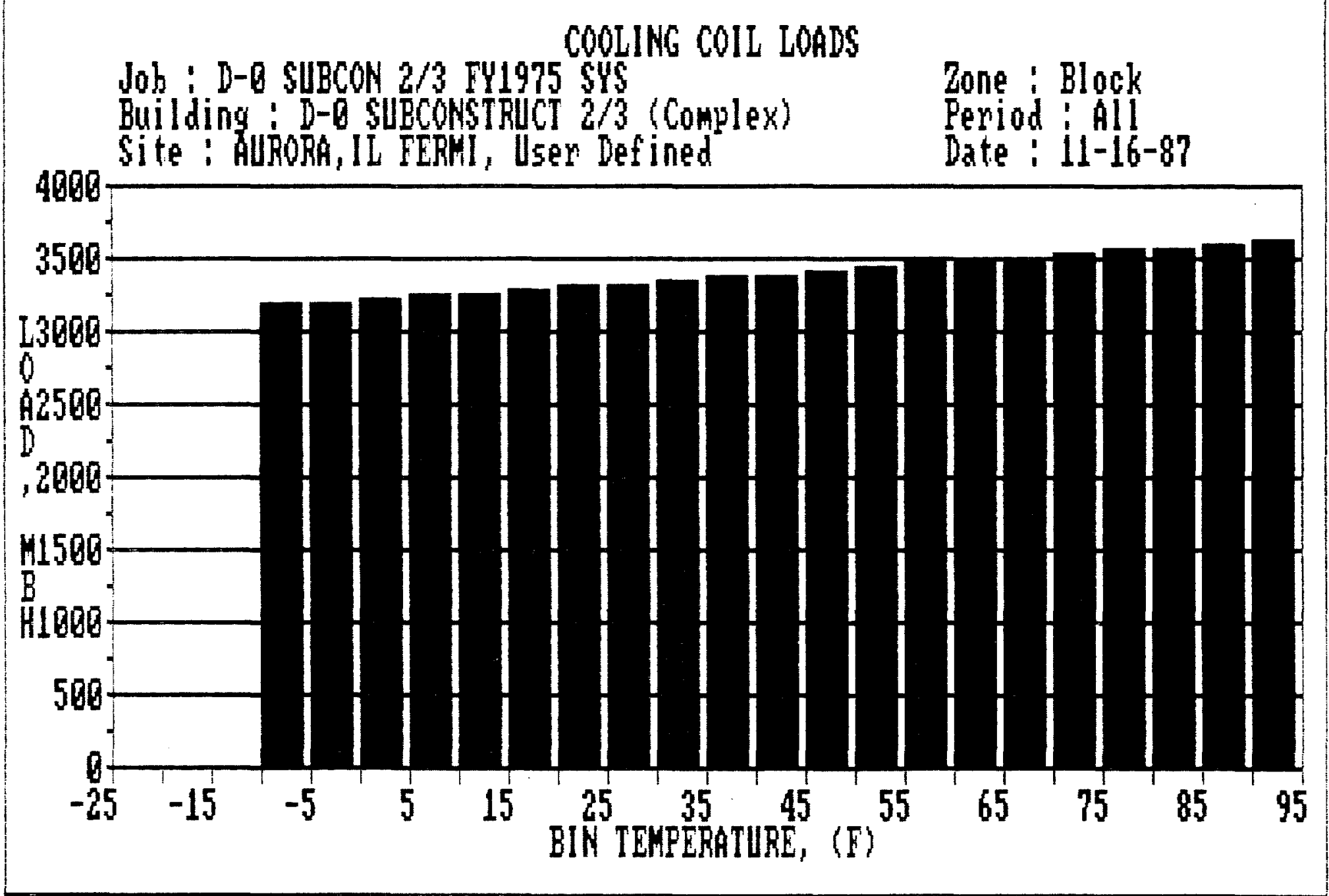




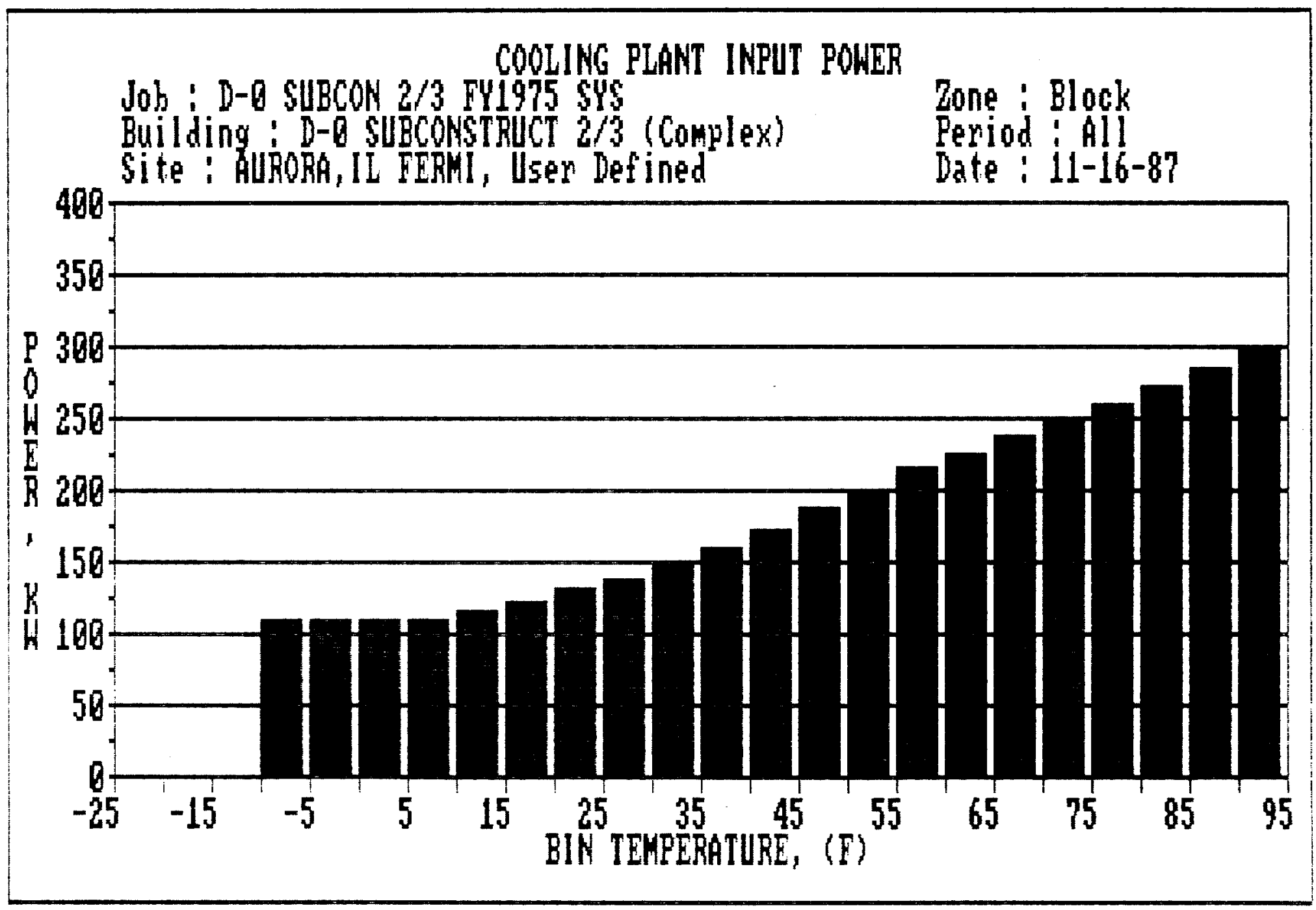




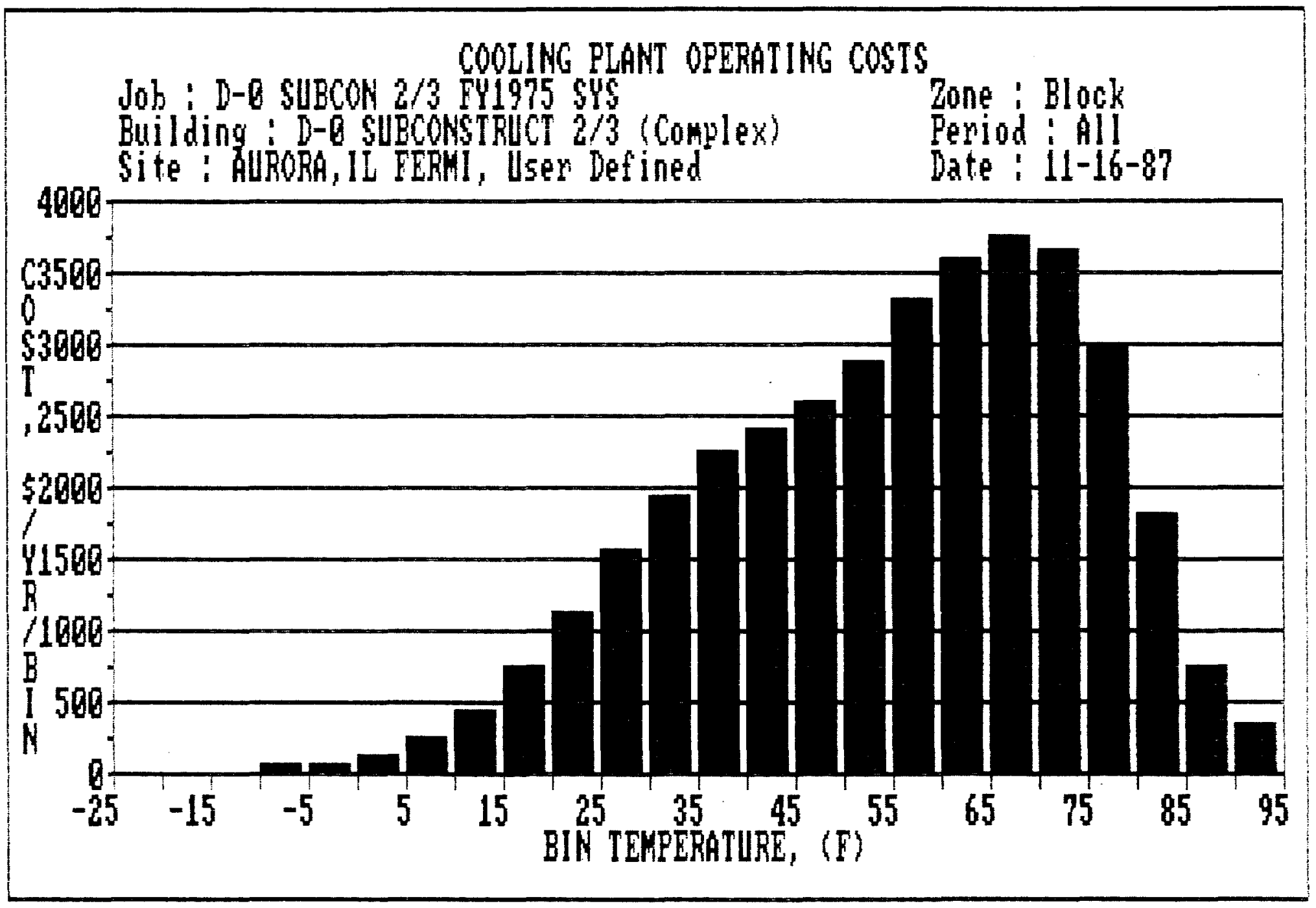




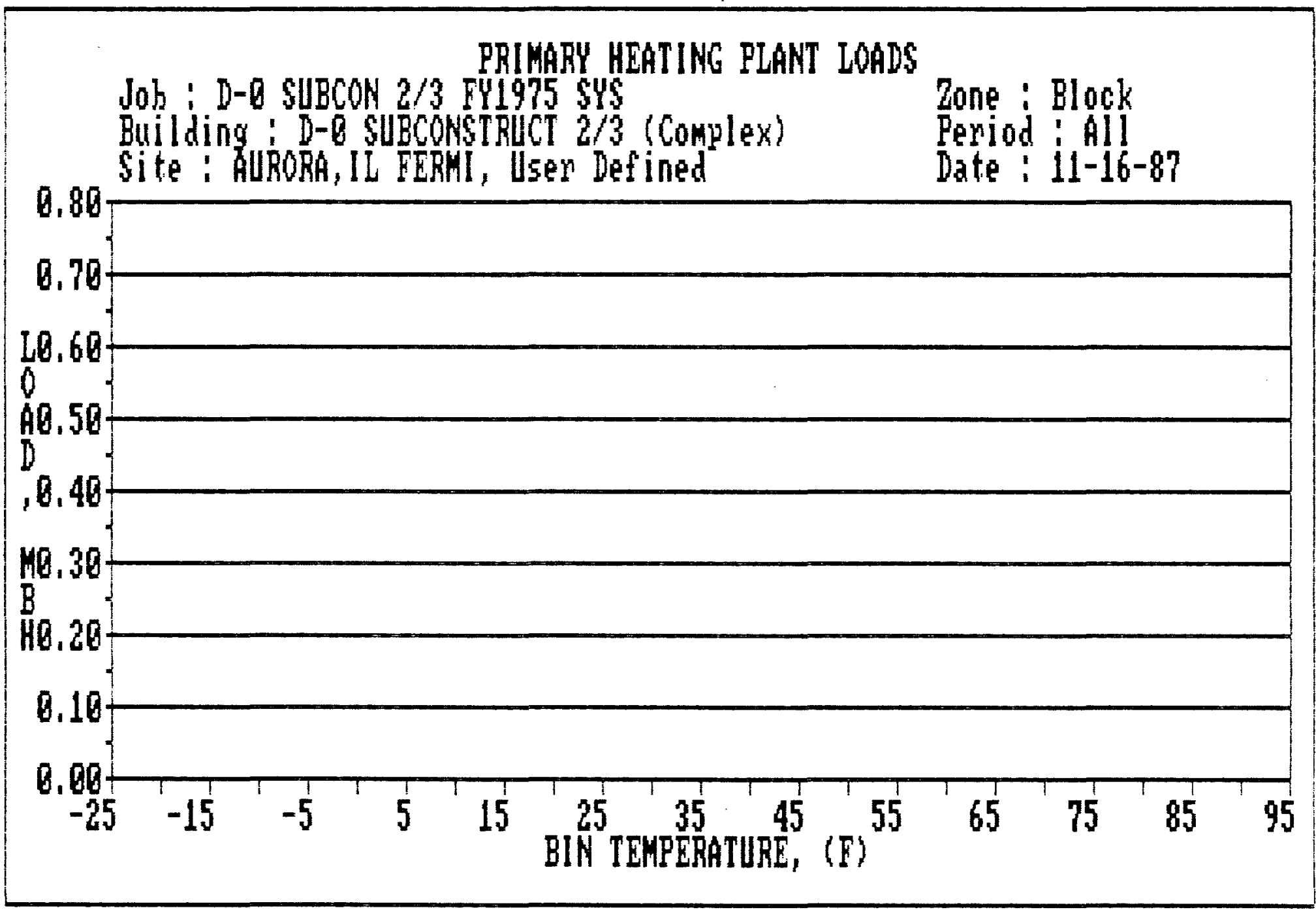


FAN OPERATING COSTS

Joh : D-O SUBCON $2 / 3$ FY1975 SUS

Building: D-0 SUBCOHSTRUCT $2 / 3$ (Complex)

Si te: AURORA, IL FERHI, User Defined

Zone: Block

Period : All

Date: 11-16-87

I

1200

01050

S

900

750

$\$ 600$

1

450

$R$

1308

B

150

N

BIN TEMPERATURE, (F) 
VI-a.

\author{
FEFMILAE D-O EXFEFIMENTAL FACILITY \\ SUECONSTFUCTION IE - LCW COOL ING SYSTEMS \\ BUTLDINE SECTOF: FHYSICAL. FAFAMETEFS AHD DESCFIFTIDN
}

3 OCTOBEF, 1987

ANALYST: S.F.FFSTULOVICH

FEFMILAE CONSTFUCTION ENGINEEFING SEFVICES

VI-1 
EuIlding Name: D-0 SUECONSTFUCT $\Xi / S$ Date: $11-16-87$ Site: AUFOFA, IL FEFMI, User- Defined 60117862.0

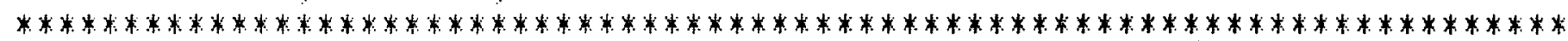
1. ROOM CONDITIONS AND FLOOF: AFEAS

Themostat settings : Cooling $=92 \mathrm{~F}$ Heating $=91 \mathrm{~F}$ Setbact $=0 \mathrm{~F}$ Warm-up factor $=0 \%$ Foom design relative humidity $=50 \%$

Floor Areas: Ferimeter $=\quad 1$ Sqfta Interior $=\quad 0$ Sqft cooling provided during unoccupied period.

2. WALLS, FOOF, GLASS, SYYLIGHTS:

$\begin{array}{ccccc} & \text { Area } & \text { U-Factor } & \text { Glass Area } & \text { Glass U-Factor } \\ \text { Exposure } & \text { (Sqft) } & \text { ETU/(hr-sqft-F) } & \text { (Sqft) } & \text { BTU/(hr-sqft-F) }\end{array}$

North Wal 1

Northeast Wall

East Wall

Southeast wall

South wall

Soutrwest Wall

West Wali

Northwest Wall

Ferimeter Foof

Interior Fioof
0.150
0.150
0.150
0.150
0.150
0.150
0.150
0.150
0.130
0.150

1.130

1.130

1.130

1. 130

1. 130

1. 130

1.1 .30

1. 150

0.550

0.550

WALLS: Color = Datk: Wt. = Medium: FOOF: Color = Medilum: Wt. = Medium EUILDING WT. = MEdiUm; VEFTICAL GLASS " Single Glazed

Shade Factors : Glass $=1.000$ : Stylights $=0.000$

Internal shades are not used. A ceiling plenum is not used.

3. LIGHTS:

Ferimeter: 0.000 Watts/5qft =

Interior : 0.000 Watts/50ft =

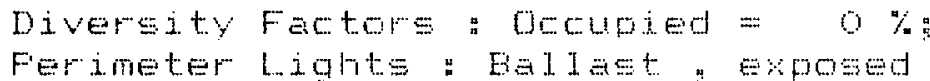

Interior lighte : Eallast "recessed

4. MISCELLANEOUS ELECTFICAL LOADS:

Ferimeter : Dccupied $=\% 600000.000$ watts/5qt $=600.000$ watts Tota

Unoccupied $=\% 200000.000$ watts/50ft $=$ 200,000 watts Tota

Interior : Dccupied $=0.000$ watts/Sqft $=$

0 Watts Total Unoccupied $=0.000$ Watts $/$ Sqft $=$

o Watts Total

5. FEOFLE LOADS

Total Dccupancy $=\quad 0$ sqft/person =

Activity Level: 6 . User defined activit

Sensible $=0.0$ ETU/hr/person: Latent $=0.0$ ETU/hr/person

Diversity Factors : Decupied $=0 \%$ : Unoccupied $=0 \%$

6. MISCELLANEOUS INTEFNAL LDADS:
Sensible: Gecupied =
O ETU/hr: Unocelpied =
O ETU/hr
Laterit a Dcupied =
0 ETU/hr: Unocempied =
O ETU/hr

7. WALLS ADJACENT TO NON-CONDITIONED FEGIONS:
Wall Areas : Ferimeter =
Wal] U-Factor $=0.000$
o squt: Interior $=$
ETU/hr-Sqft-F)

Adjacent region is not heated

Adjecent region is not cooled

8. INFILTFATION DATA:

Air Flow Fates: Occupied = VI-2

o efin: Unaceupied =:

offm 
VI-b.

\author{
FEFMILAE DOO EXFEFIMENTAL FACILITY \\ SUECONSTFUCTION $\because / S$ - LCW COOLING SYSTEMS \\ EUILDINO LOAD CALCULATIONS \\ I OCTDEEF, 1787 \\ ANALYST: S.F.HFSTULOUICH \\ FEFMILAS CONSTFUCTION ENGINEEFING SEFUICES
}

VI-3 
LDAD FFIFILE SUMMAFY

Building Name : D-O SUECONSTRUCT $3 / 3$ (Complex)

Date : $11-16-87$

Site Name : AUFOFA, IL FEFivi, USer Defined

60117862.0

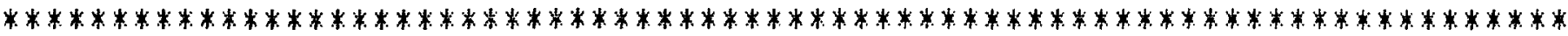

TAELE 1. OCCUFIED FEFIOD (Load does not iniclude vent. load, fan heat)

\begin{tabular}{|c|c|c|c|c|c|c|c|c|c|}
\hline Ein & Ein & $--\cdots-F$ & arimeter & Zone--.- & ---3 & - & Interior & Zone- & --9 \\
\hline $\begin{array}{l}\text { Temp } \\
\text { (F) }\end{array}$ & $\begin{array}{l}\text { Hours } \\
\text { (hr/yr) }\end{array}$ & $\begin{array}{c}\text { Sensible } \\
\text { (tons) }\end{array}$ & $\begin{array}{l}\text { Letent } \\
\text { (tons) }\end{array}$ & $\begin{array}{l}\text { Flenum } \\
\text { (tans) }\end{array}$ & $\begin{array}{l}\text { Zorie } \\
\text { (F) }\end{array}$ & $\begin{array}{c}\text { Sensitie } \\
\text { (tons) }\end{array}$ & $\begin{array}{l}\text { Latent } \\
\text { (tons) }\end{array}$ & $\begin{array}{l}\text { Fienum } \\
\text { (tons) }\end{array}$ & $\begin{array}{l}\text { Zone } \\
\text { (F) }\end{array}$ \\
\hline 93 & 0.0 & 170.65 & 0.00 & 0.00 & 92 & 0.00 & 0.00 & 0.00 & - \\
\hline 90 & 25.8 & 170.65 & 0.00 & 0.00 & 92 & 0.00 & 0.00 & 0.00 & - \\
\hline 85 & 59.4 & 170.65 & 0.00 & 0.00 & 92 & 0.00 & 0.00 & 0.00 & - \\
\hline 80 & 145.3 & 170.65 & 0.00 & 0.00 & 92 & 0.00 & 0.00 & 0.00 & - \\
\hline 75 & 237.7 & 170.65 & 0.00 & 0.00 & 92 & 0.00 & 0.00 & 0.00 & - \\
\hline 70 & 270.6 & 170.65 & 0.00 & 0.00 & 92 & 0.00 & 0.00 & 0.00 & - \\
\hline 65 & 264.5 & 170.65 & 0.00 & 0.00 & 92 & 0.00 & 0.00 & 0.00 & -- \\
\hline 60 & 255.0 & 170.65 & 0.00 & 0.00 & 92 & 0.00 & 0.00 & 0.00 & -1 \\
\hline 55 & 244.7 & 170.65 & 0.00 & 0.00 & 92 & 0.00 & 0.00 & 0.00 & -1 r \\
\hline 50 & 232.1 & 170.65 & 0.00 & 0.00 & 92 & 0.00 & 0.00 & 0.00 & -- \\
\hline 45 & 229.5 & 170,65 & 0.00 & 0.00 & 92 & 0.00 & 0.00 & 0.00 & -- \\
\hline 40 & 237.6 & 170.65 & 0.00 & 0.00 & 92 & 0.00 & 0.00 & 0.00 & -- \\
\hline 35 & 230.5 & 170.65 & 0.00 & 0.00 & 92 & 0.00 & 0.00 & 0.00 & -- \\
\hline 30 & 210.2 & 170.65 & 0.00 & 0.00 & 92 & 0.00 & 0.00 & 0.00 & -- \\
\hline 25 & 174.5 & 170.65 & 0.00 & 0.00 & 92 & 0.00 & 0.00 & 0.00 & - \\
\hline 20 & 122.4 & 170.65 & 0.00 & 0.00 & 92 & 0.00 & 0.00 & 0.00 & -- \\
\hline 15 & 78.1 & 170.65 & 0.00 & 0.00 & 92 & 0.00 & 0.00 & 0.00 & - \\
\hline 10 & 46.1 & 170.65 & 0.00 & 0.00 & 92 & 0.00 & 0.00 & 0.00 & -1 \\
\hline 5 & 25.1 & 170.65 & 0.00 & 0.00 & 92 & 0.00 & 0.00 & 0.00 & -1 \\
\hline 0 & 12.7 & 170.65 & 0.00 & 0.00 & 92 & 0.00 & 0.00 & 0.00 & - \\
\hline-5 & 6.2 & 170.65 & 0.00 & 0.00 & 92 & 0.00 & 0.00 & 0.00 & - \\
\hline-6 & 4.6 & 170.65 & 0.00 & 0.00 & 92 & 0.00 & 0.00 & 0.00 & -- \\
\hline
\end{tabular}

TABLE 2. UNOCCUFIED FEFIOD (Loed does not include vent. load, fan heat)

\begin{tabular}{|c|c|c|c|c|c|c|c|c|c|}
\hline 90 & 14.6 & $56.8 \mathrm{e}$ & 0.00 & 0.00 & 92 & 0.00 & 0.00 & 0.00 & - \\
\hline 85 & 36.3 & $56.8 \mathrm{\theta}$ & 0.00 & 0.00 & 72 & 0.00 & 0.00 & 0.00 & -- \\
\hline 80 & 99.3 & 56.89 & 0.00 & 0.00 & 92 & 0.00 & 0.00 & 0.00 & -- \\
\hline 75 & 215.3 & 56.88 & 0.00 & 0.00 & 92 & 0.00 & 0.00 & 0.00 & - \\
\hline 70 & 357.6 & 56.80 & 0.00 & 0.00 & 92 & 0.00 & 0.00 & 0.00 & - \\
\hline 65 & 463.3 & 56.88 & 0.00 & 0.00 & 92 & 0.00 & 0.00 & 0.00 & - \\
\hline 60 & 500.4 & 56.88 & 0.00 & 0.00 & 92 & 0.00 & 0.00 & 0.00 & -- \\
\hline 55 & 482.0 & 56.88 & 0.00 & 0.00 & 92 & 0.00 & 0.00 & 0.00 & $-\cdots$ \\
\hline 50 & 447.6 & 56.88 & 0.00 & 0.00 & 92 & 0.00 & 0.00 & 0.00 & -- \\
\hline 45 & 422.7 & 56.88 & 0.00 & 0.00 & 92 & 0.00 & 0.00 & 0.00 & -- \\
\hline 40 & 41.5 & 56.88 & 0.00 & 0.00 & 92 & 0.00 & 0.00 & 0.00 & -- \\
\hline 55 & 417.0 & 56.88 & 0.00 & 0.00 & 92 & 0.00 & 0.00 & 0.00 & $\ldots$ \\
\hline 30 & 417.4 & 56.80 & 0.00 & 0.00 & 92 & 0.00 & 0.00 & 0.00 & $-\cdots$ \\
\hline 25 & 395.2 & 56.88 & 0.00 & 0.00 & 92 & 0.00 & 0.00 & 0.00 & - \\
\hline 20 & 3.6 .9 & 56.88 & 0.00 & 0.00 & 92 & 0.00 & 0.00 & 0.00 & -- \\
\hline 15 & 252.8 & 56.88 & 0.00 & 0.00 & 92 & 0.00 & 0.00 & 0.00 & -- \\
\hline 10 & 165.7 & 56.88 & 0.00 & 0.00 & 92 & 0.00 & 0.00 & 0.00 & $\cdots$ \\
\hline 5 & 96.5 & 56.80 & 0.00 & 0.00 & 92 & 0.00 & 0.00 & 0.00 & - \\
\hline 0 & 50.9 & 56.88 & 0.00 & 0.00 & 72 & 0.00 & 0.00 & 0.00 & -- \\
\hline-5 & 25.3 & 56.88 & 0.00 & 0.00 & 92 & 0.00 & 0.00 & 0.00 & - \\
\hline-6 & 21,1 & 56.80 & 0.00 & 0.00 & 92 & 0.00 & 0.00 & 0.00 & $\cdots$ \\
\hline-6 & 0.0 & 0.00 & 0.00 & 0.00 & 91 & 0.00 & 0.00 & 0.00 & - \\
\hline
\end{tabular}


VI-C.

\author{
FEFMILAE DWO EXFEFIMENTAL FACTLITY \\ SUEOONETFUCTION $\because \%$ - LCW COOLINE SYSTEMS \\ SYSTH COMFARSON FEFWOHANCE SIMUIATHONS \\ 1 OCTOEEF, 1987 \\ ANALYS: SF.FFTULOUYCH \\ FEFMILAE CONOTFUETION ENGINEFFTNG EEFUTCES
}




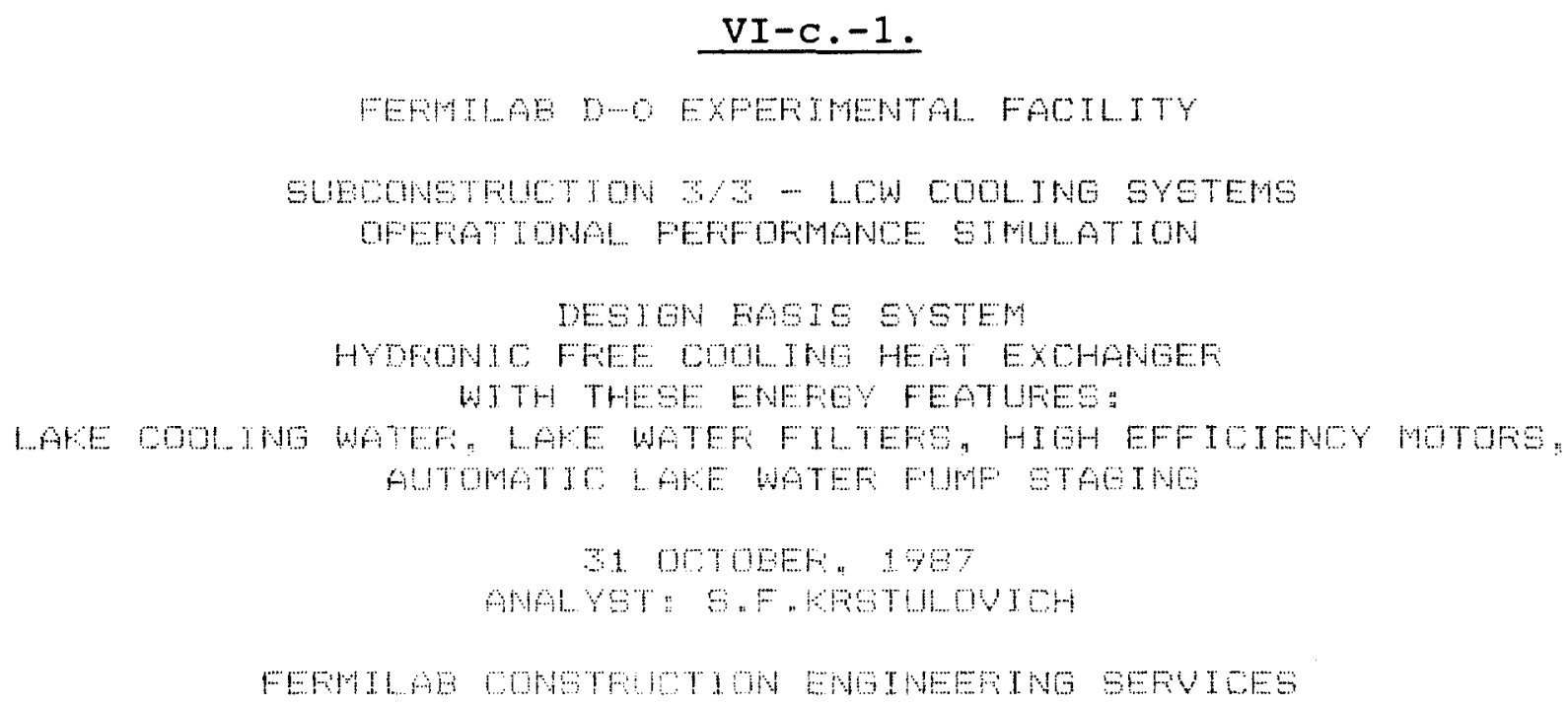




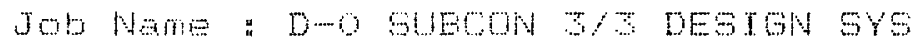

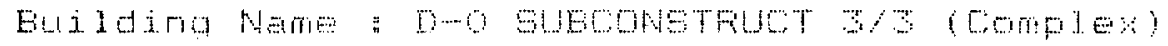

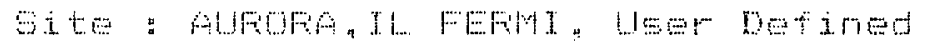

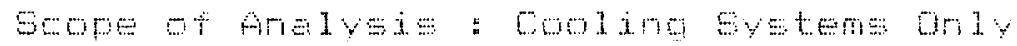

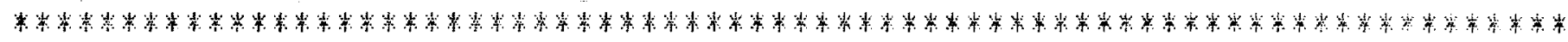

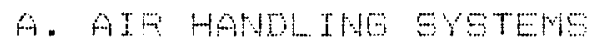

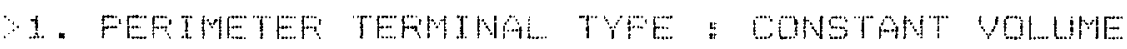

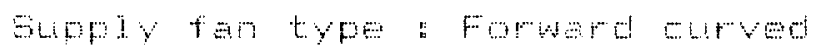

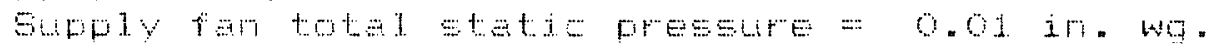

A return fan d. not lsed.

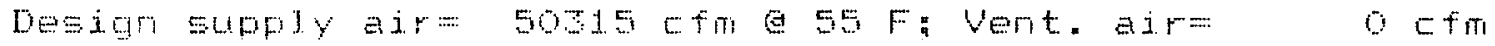

Are woldng terminal: Lsed for heatino ? Y

An Ecomonizer is not lised.

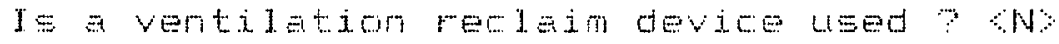

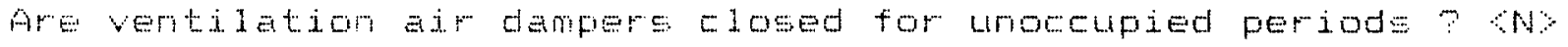

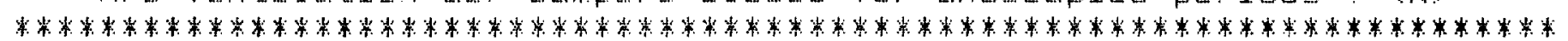
E. HEATING FLANTG

1. FEFIMTEF FLAN : ELELTFECL FESISTANCE

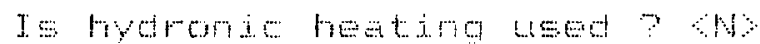

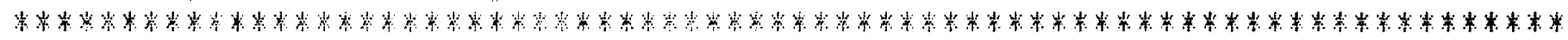




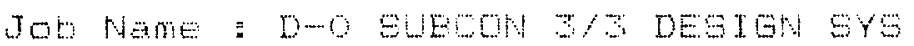

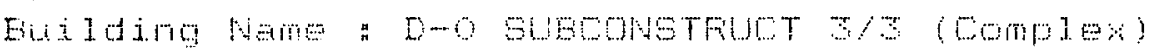

Fige 2

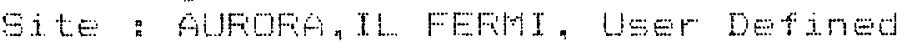

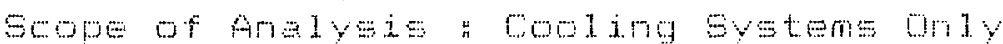

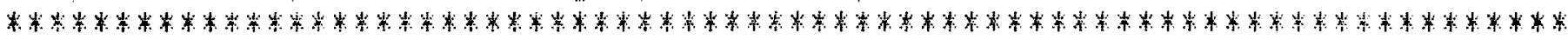

C. GOTH. ING FHATS

1. FEFTMETEF FLAUT : FEMUTE SULFEE COULING

Is rydronie cooling used $\%$ Y Y

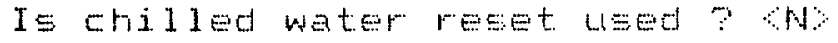

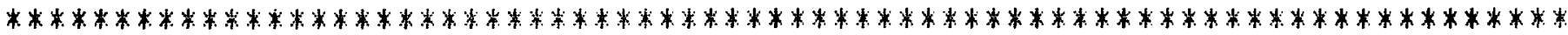

D. FUMFIMG SYSTEMS

\begin{tabular}{|c|c|c|c|c|c|c|c|c|c|}
\hline I & & $\vdots$ & & Ni: & $T E F$ & ! & & & \\
\hline : & System & $i$ & $d T, F$ & 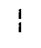 & ft wo & i & $d T, F$ & $: d F$ & ft $w g$ \\
\hline 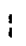 & Chil led water & i & 20.00 & $i$ & 150.00 & i & 0.00 & i & 0.00 \\
\hline ! & Hot water & $i$ & 0.00 & $\mathrm{i}$ & 0.00 & i & 0.00 & $i$ & 0.00 \\
\hline & Condenser weter & i & 0.00 & $\frac{1}{1}$ & 0.00 & : & 0.00 & $i$ & 0.00 \\
\hline & Ground weter & $i$ & $\mathrm{H}_{n} \mathrm{Or}$ & 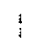 & 0.00 & $i$ & 0.00 & $i$ & 0.00 \\
\hline
\end{tabular}

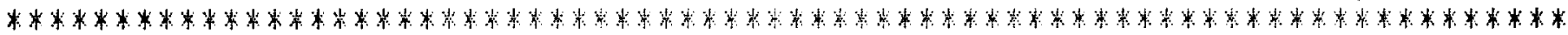

E. NON-HWA $g$ YTEM

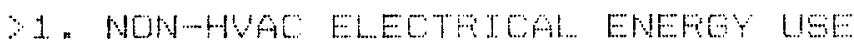

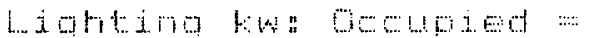
o.
Y.

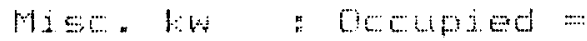
bo a
$200.0 \quad 14$
other ta : actipied =
o.

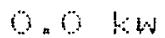

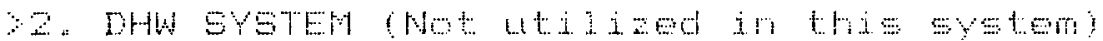

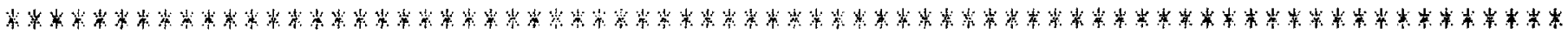
F. FUEL CDE

\begin{tabular}{|c|c|c|c|c|c|c|c|c|}
\hline$\because 1$ & 1 & \multicolumn{3}{|c|}{ 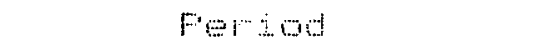 } & $i$ & $\because 3.0 H E F$ FLES & i & Writ $\operatorname{Cos}$ \\
\hline ELEOTFICAL & $\vdots$ & $90 E$ LIEI & & (lom & $i$ & 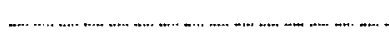 & & (n) \\
\hline EMEFGY UEE & $i$ & $(\div)+164$ & $\vdots$ & $(t+1,1)$ & $i$ & 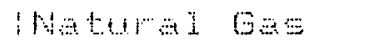 & $i$ & ifthern \\
\hline 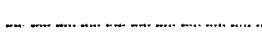 & 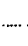 & 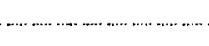 & & 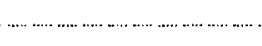 & $\cdots .$. & $\because 401 \quad 01]$ & i & $q ; 0,6$, 101 \\
\hline Conpre $=0$ mir & $\vdots$ & 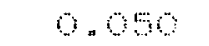 & $\vdots$ & $\gamma=\hat{y}$ & 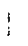 & Fropв & $\vdots$ & $4 / 1 \mathrm{~b}$ \\
\hline FEI $=$ tame & $\vdots$ & $0,0,0$ & & $0,9,1$ & $\vdots$ & 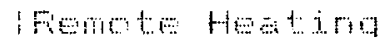 & $\vdots$ & $\leftrightarrow / \mathrm{PH}$ \\
\hline Th土tetive & $i$ & 9.9 & $\vdots$ & i. . & $i$ & Finote Eno I imo & $i$ & 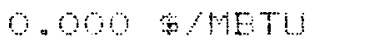 \\
\hline
\end{tabular}


TOTAL EUTLDING ENEFOY SUMMATY

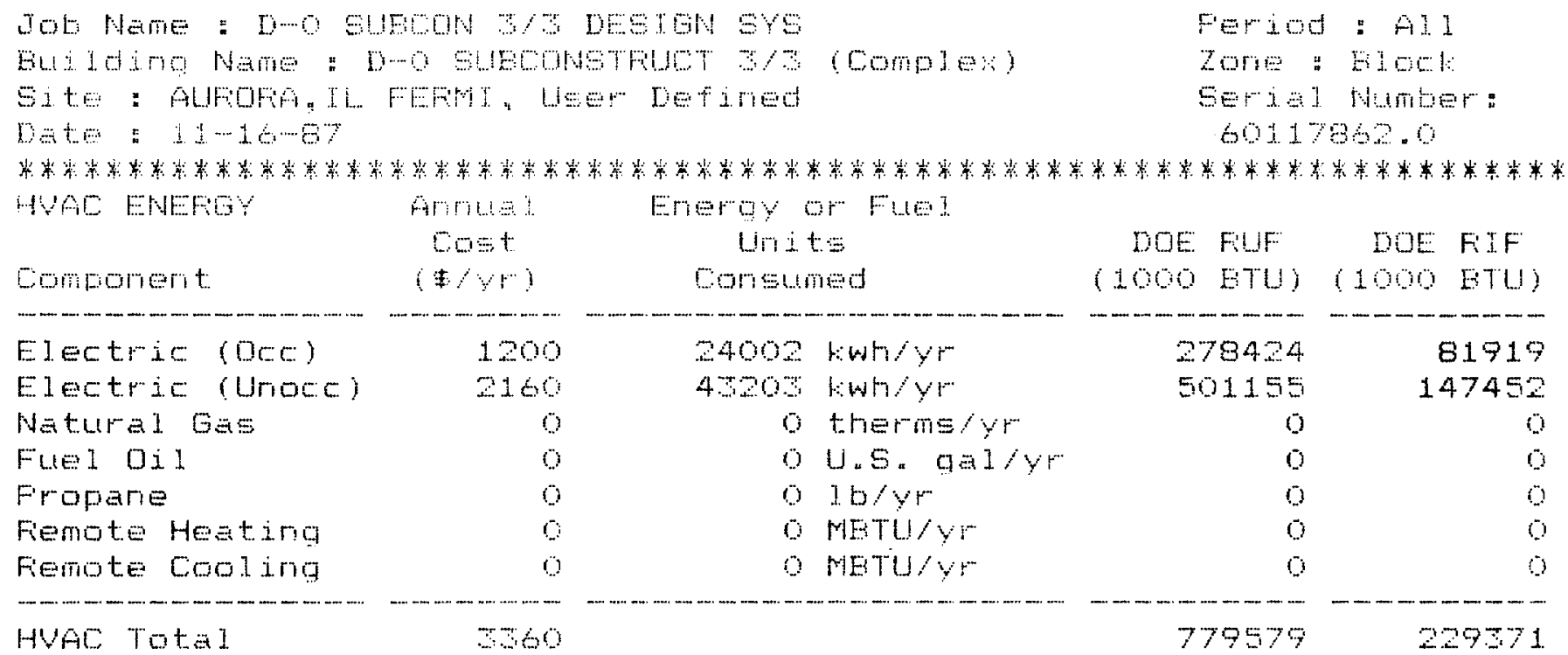

HONHWAE ENEFEY

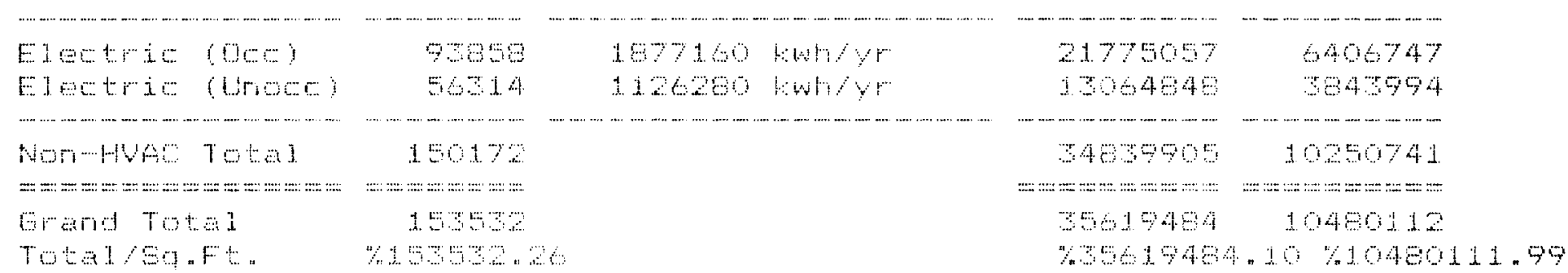

HYo sumary

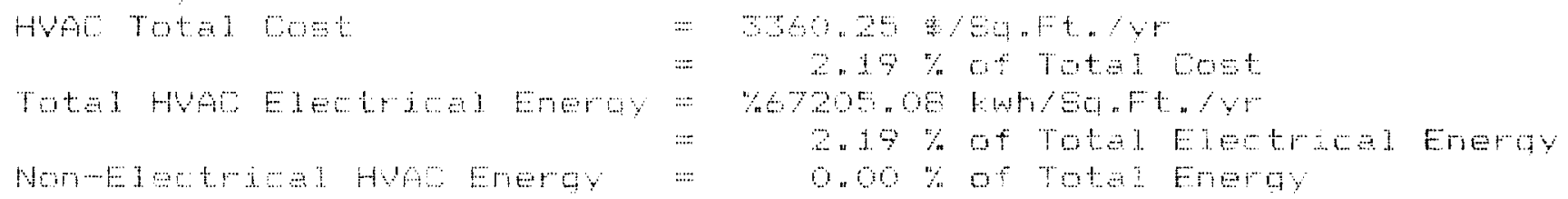

rey:

\begin{tabular}{|c|c|}
\hline fats & $=11600$ HU FuF ; 342 equ \\
\hline thern nat $a=0$ & $=100000$ BU fur and Fif \\
\hline $1.8 .90 \%$ का & 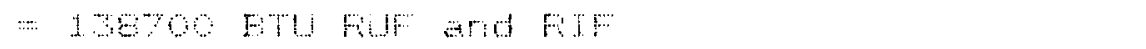 \\
\hline $\operatorname{lng} 0.0101$ & $=169000$ BTU FUF and FIF \\
\hline it propene & $=21680$ BTU FUF and FIF \\
\hline U. & $=$ Fesoumer utilization faotor (Source value) \\
\hline If & $=$ Fesoumee impact factor (foint of use value) \\
\hline $\mathrm{BH}$ & $=1000$ ETU/FF \\
\hline & $=$ MLIIIOn ETU \\
\hline
\end{tabular}




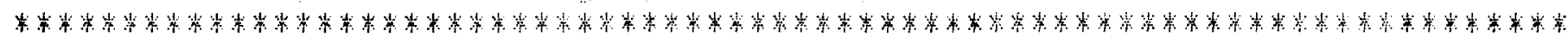

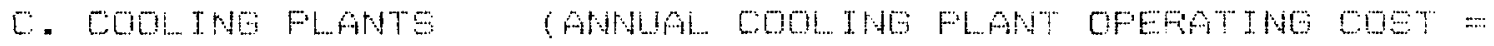

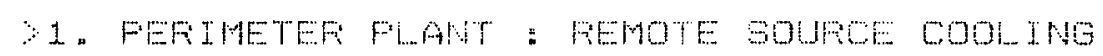
I B hydronic cooling lised $?$

Is chilled water reset lsed ? $\mathrm{N}$

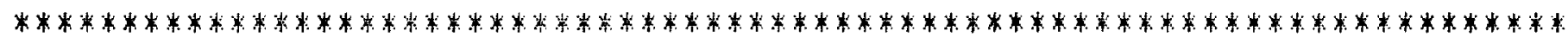

D. FUMFING SYSTEMS

(ANNUAL FUMF OFEFATTNG COST = $\$, 12$ )

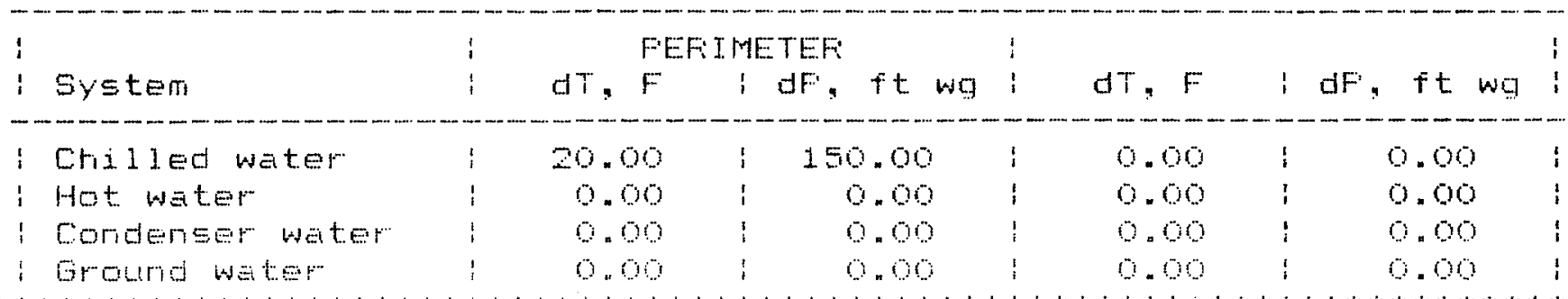

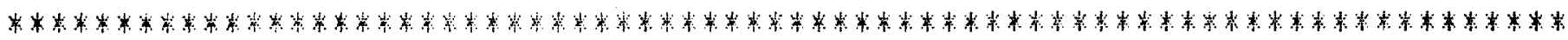

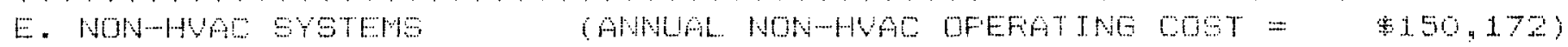

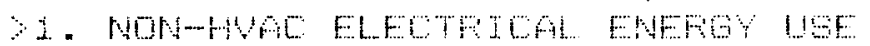

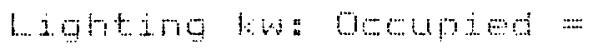
a
3. 10 in

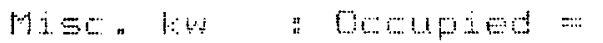
की व
$\therefore 0 \%$ क

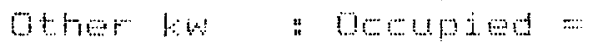

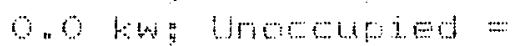
in 1 \%

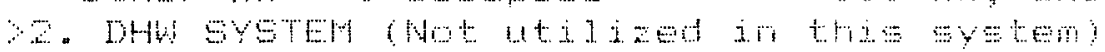

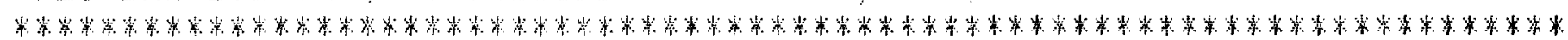

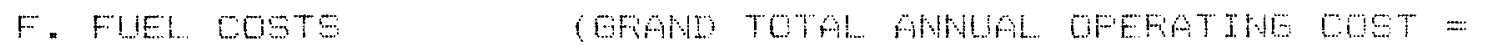

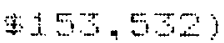

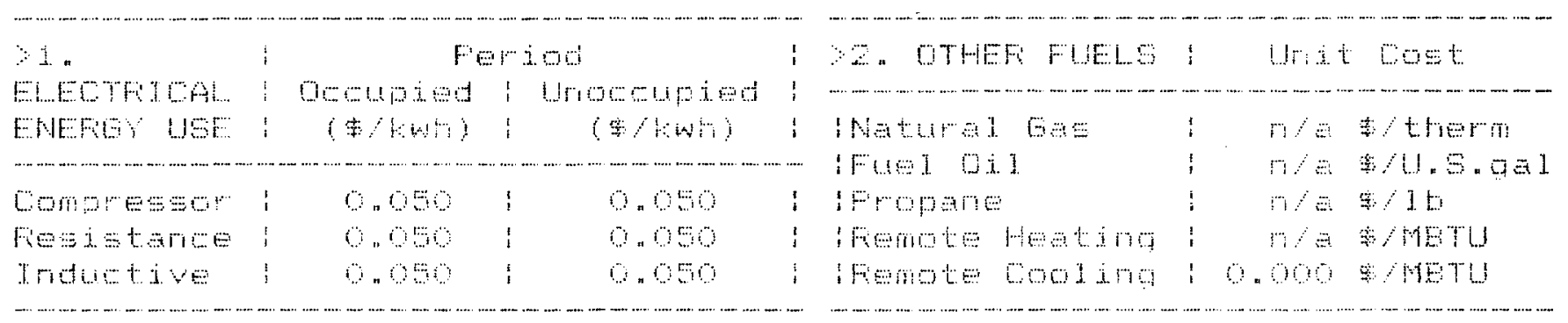




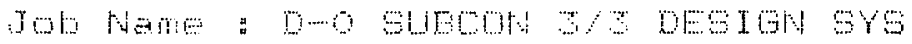

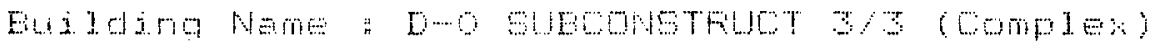

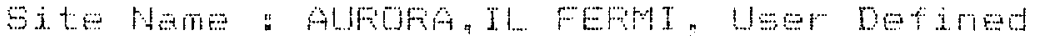

Date: $11-16-87$

Eeriaj Numbe: 60176620

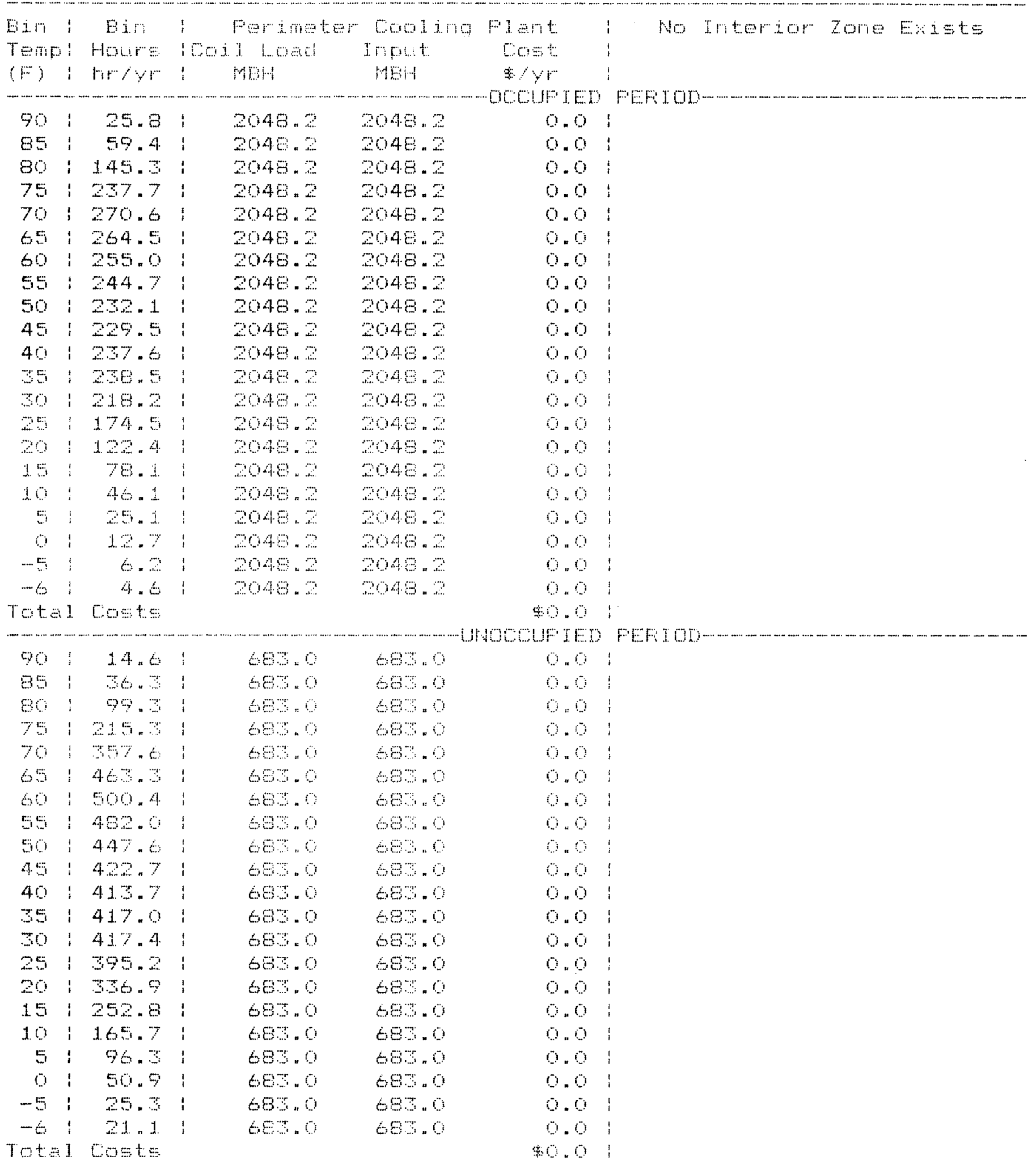


FAM AND FUM CoST

Jot Nan: : D-O SUECON 23 DECION $9 \mathrm{Y}$

Euilding Name " D-O SUEOUNSTFUCT $\Xi / Z$ (Commlex)

Site Name : AUFOFA, IL FEFM , UEE Detined

Date: $11-16-87$

Seri.i. Number: $6117 \operatorname{sen} 0$

\begin{tabular}{|c|c|c|c|c|c|c|c|c|c|c|}
\hline $\begin{array}{l}\text { Ein } \\
\text { TEmp } \\
(\mathrm{F})\end{array}$ & & $\begin{array}{l}\text { Ein } \\
\text { Hours } \\
\text { hr/y }\end{array}$ & $\vdots$ & $\begin{array}{l}\text { Ferineter } \\
\text { mont } \\
\text { ful }\end{array}$ & $\begin{array}{l}\text { Fans } \\
\text { cost } \\
\text { कy }\end{array}$ & : No & Interior zorie & 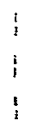 & $\begin{array}{l}\text { Al] } \\
\operatorname{Input} \\
\text { fla! }\end{array}$ & $\begin{array}{l}\text { Fumpe } \\
\text { Cost } \\
\text { wy }\end{array}$ \\
\hline 90 & : & 25.8 & i & 0.1 & 0.1 & i & EFitu & $i$ & 7.6 & 9.8 \\
\hline 85 & 1 & 59.4 & $i$ & 0.1 & 0.2 & 1 & & i & 7.6 & 22.5 \\
\hline 80 & $!$ & 145,3 & $i$ & 0.1 & 0.8 & 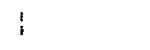 & & $i$ & 7.6 & 54.9 \\
\hline 75 & 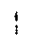 & 27.7 & $:$ & 0.1 & $1 . Z$ & 1 & & 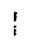 & 7.6 & 89.9 \\
\hline 70 & i & 270.6 & 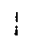 & 0.1 & 1.5 & $i$ & & $:$ & 7.6 & 102.3 \\
\hline 65 & $i$ & 264.5 & ! & 0.1 & 1.4 & ! & & $i$ & 7.6 & 100,0 \\
\hline 60 & 3 & 255.0 & $\vdots$ & 0.1 & 1. 4 & : & & $i$ & 7.6 & 56.4 \\
\hline 55 & $i$ & 244.7 & 1 & 0.1 & 1.3 & $!$ & & $!$ & 7.6 & 92.5 \\
\hline 50 & i & 22.1 & 1 & 0.1 & 1.5 & $!$ & & $i$ & 7.6 & 87.8 \\
\hline 45 & $i$ & 229.5 & $\vdots$ & 0.1 & 1.3 & $i$ & & $i$ & 7.6 & 86.8 \\
\hline 40 & $!$ & $27 \%$ & $!$ & 0.1 & 1.3 & $!$ & & 1 & 7.6 & 89.8 \\
\hline$r$ & 1 & 24.5 & $\frac{1}{i}$ & 0.1 & 1.5 & : & & 1 & 7.6 & 90.2 \\
\hline 50 & $\vdots$ & 210.2 & 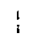 & 0.3 & 1.2 & $!$ & & $i$ & 7.6 & 82.5 \\
\hline 25 & $i$ & 174.5 & $i$ & 0.1 & 1.0 & : & & 1 & 7.6 & 60.0 \\
\hline 20 & $\vdots$ & 122,4 & $\vdots$ & 0.1 & $0_{4} 7$ & 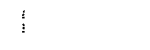 & & $i$ & 7.6 & $46 .$. \\
\hline$\dot{25}$ & $\vdots$ & 78.1 & $i$ & 0.1 & 0.4 & : & & 1 & 7.6 & 29.5 \\
\hline 10 & $i$ & 46.1 & 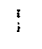 & $\therefore 1$ & \% & $i$ & & $i$ & 7.8 & 17.4 \\
\hline 5 & $\vdots$ & 25.1 & $\vdots$ & 0.5 & $=i$ & $i$ & & $i$ & 7.6 & 9.5 \\
\hline o & $i$ & 12.7 & $\vdots$ & 0.1 & 3.1 & $i$ & & $i$ & 7.6 & 4.8 \\
\hline-5 & $\vdots$ & 6.2 & 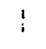 & $n 1$ & . & $:$ & & $\grave{\imath}$ & $7 .=$ & 2.3 \\
\hline$-\ldots$ & $\vdots$ & 4.6 & $\vdots$ & 0.1 & i & $i$ & & $i$ & 7.6 & 1.7 \\
\hline Teto & 1 & $0=$ & & & 317.1 & $i$ & & $\vdots$ & & \$192.0 \\
\hline 9 & $\vdots$ & 14.6 & ! & a. & 0.1 & $\begin{array}{l}\text { Nomedu } \\
\vdots\end{array}$ & $x, P=10$ & 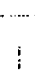 & 7.5 & 55 \\
\hline 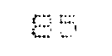 & $i$ & के & $i$ & . & 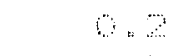 & 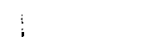 & & i & 7.6 & 1.7 \\
\hline 8 & : & 94 & $\vdots$ & , & 0 & : & & $!$ & 7. & 37.5 \\
\hline 75 & $\vdots$ & 215,3 & $!$ & 1.1 & 1,2 & $i$ & & i & 7.6 & 81.4 \\
\hline \% & $i$ & 59. & $\vdots$ & 1.1 & $\therefore 0$ & $:$ & & 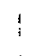 & 7.6 & 105 \\
\hline 65 & $\vdots$ & $46 n$ & $i$ & . & 2. & : & & i & 7.5 & $175 \cdot 2$ \\
\hline 60 & $\vdots$ & अण 4 & $\vdots$ & 0,1 & 27 & $\vdots$ & & 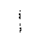 & 7.6 & 169 \\
\hline 5 & $i$ & 40 & $\vdots$ & 0.1 & $2: 3$ & 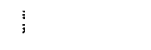 & & $!$ & 7.6 & $18 x, 3$ \\
\hline 50 & $\mathrm{i}$ & $44 \% .6$ & $\vdots$ & 0.1 & $2: 4$ & i & & : & $\because \ldots$ & 169.5 \\
\hline 45 & 1 & $42=7$ & 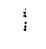 & $a . i$ & 2 & i & & $\vdots$ & 7.6 & 159.8 \\
\hline 40 & $!$ & 412,7 & $\mathfrak{\imath}$ & 0.1 & 2.3 & i & & 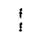 & 7.6 & 156.4 \\
\hline 35 & $\dot{3}$ & $41 \%$ & $i$ & 0.1 & 23 & $\dot{i}$ & & : & 7.6 & $167 \%$ \\
\hline 3 & $\vdots$ & $417 \times 4$ & $i$ & $\mathrm{U}_{n}$ & 2,3 & i & & $i$ & 7.6 & 157.8 \\
\hline 25 & $i$ & 593.2 & $!$ & 0.1 & 2. & : & & $i$ & 7.6 & 149.4 \\
\hline 20 & $\vdots$ & 769 & $!$ & 1,1 & 1.6 & i & & i & 7.6 & $1: 7.4$ \\
\hline 15 & $!$ & 2120 & 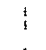 & 0,1 & 1.4 & $i$ & & 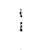 & 7.6 & 90.6 \\
\hline 10 & $!$ & $165 \times 7$ & $i$ & . 1 & 1.9 & 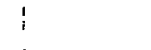 & & $\vdots$ & 7.6 & 62.7 \\
\hline 5 & $i$ & 96.3 & $!$ & 0.1 & 0.5 & $i$ & & 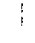 & 7.6 & 54 \\
\hline 0 & : & $50 \%$ & $\mathrm{i}$ & .1 & 0.8 & $\vdots$ & & i & 7.6 & $19 \cdot 2$ \\
\hline-5 & 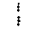 & 25,5 & $i$ & 01 & 0.1 & $\dot{1}$ & & 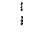 & 7.6 & 96 \\
\hline-6 & $i$ & 21 - 1 & : & 0.1 & 1 & $\vdots$ & & i & 7.6 & $\theta .0$ \\
\hline Tote & & $\cos +\infty$ & & & ब्र & 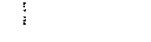 & & $:$ & & 3.129 .4 \\
\hline
\end{tabular}


COOLING COIL LOADS

Joh : D-O SUBCON $3 / 3$ DESIGN SYS

Building : D-6 SUBCONSTRUCT $3 / 3$ (Complex)

Zone: Block

Site : AURORA, IL FERHI, User Def ined

Period: All

4000

3500

13800

0

A2500

D)

2600

$\mathrm{H} 1560$

B

H1000

560

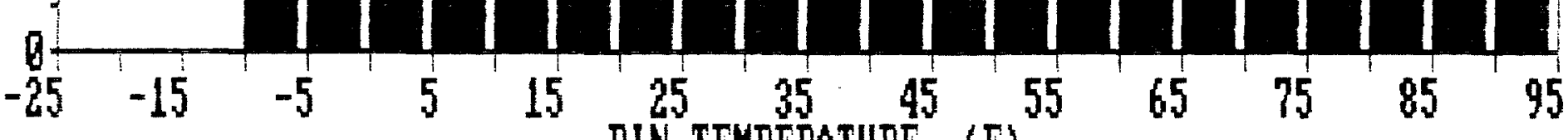

BIN TEMPERATURE, (F) 


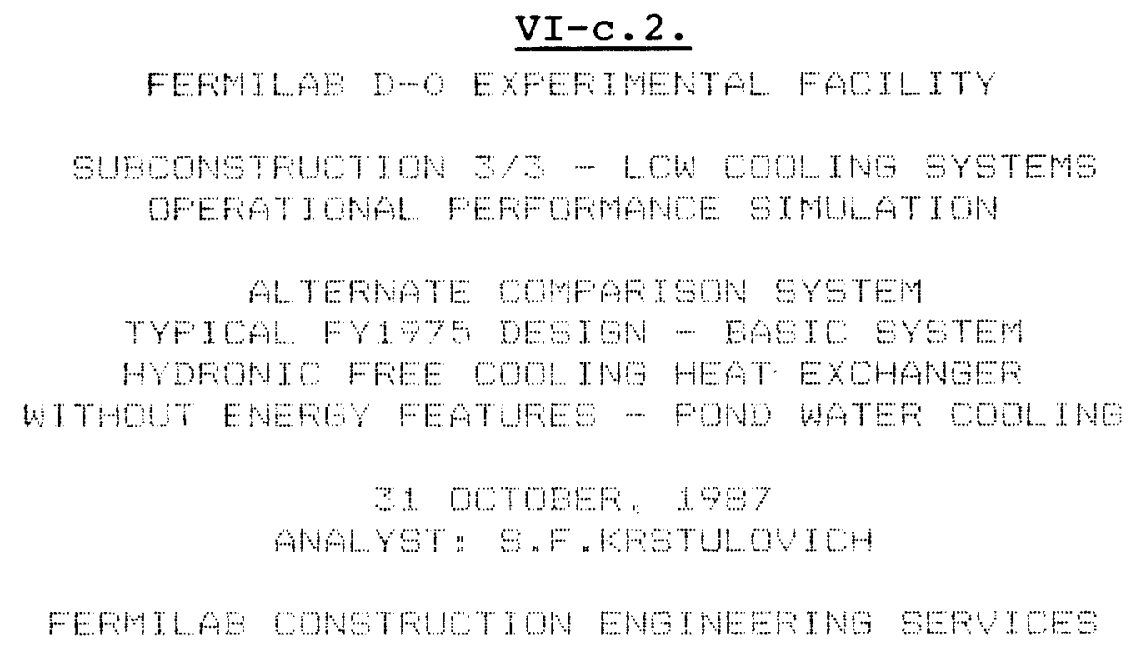




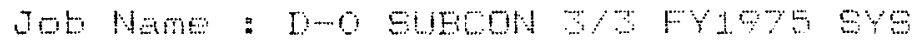

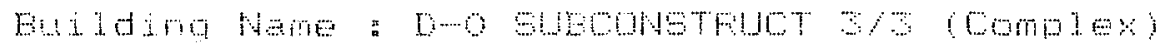

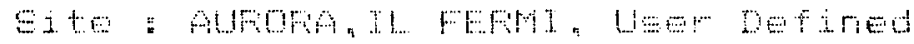

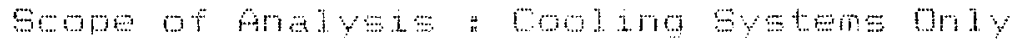

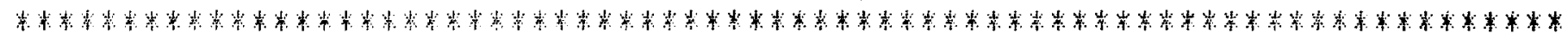

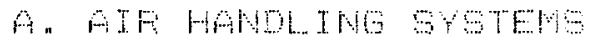

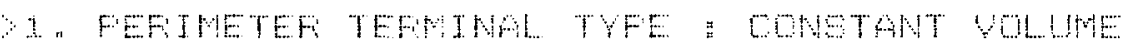

Siply tan trim

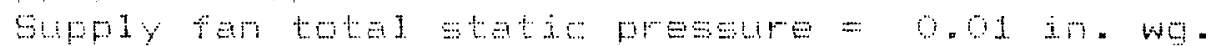

A return tan is mot wata

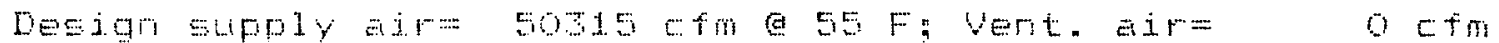

Are cowling teminale used tor heating ? y

Ar exomomizer is not used.

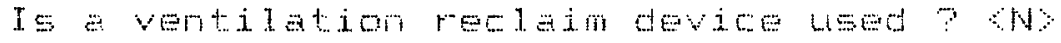

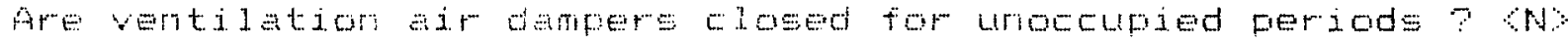

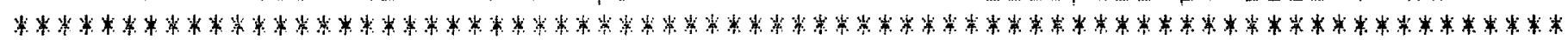

E. HEATING FLANTG

$\vartheta$ ¿. "FEFIMETEF FLANT " EIELTFICAL FESTETANCE

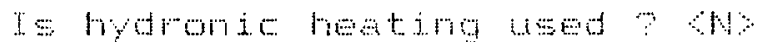

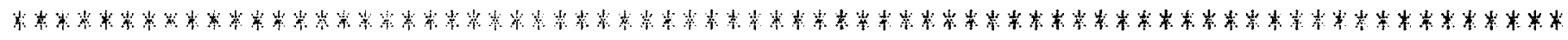


Tot Name: Do SubCon

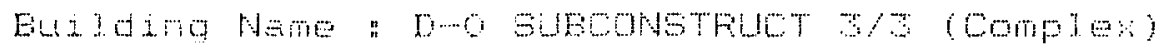

$F$ Fae 2

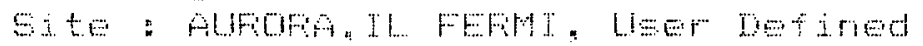

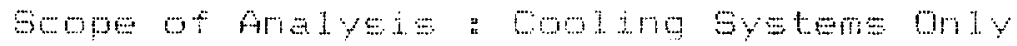

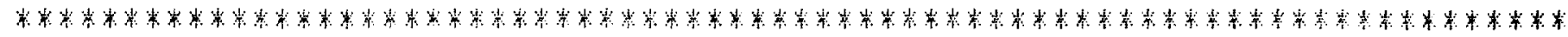

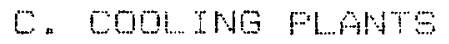

1. FEFIMETEF FLAN" : FEMOTE OUURE COOLING

Is hydronie cooling used $?$ Y

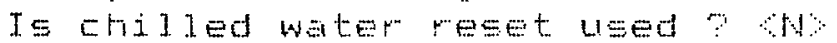

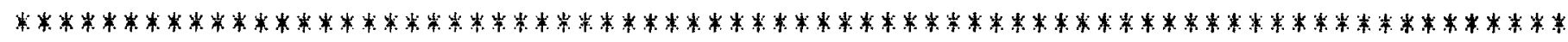

D. FUMFINE SYSTEMS

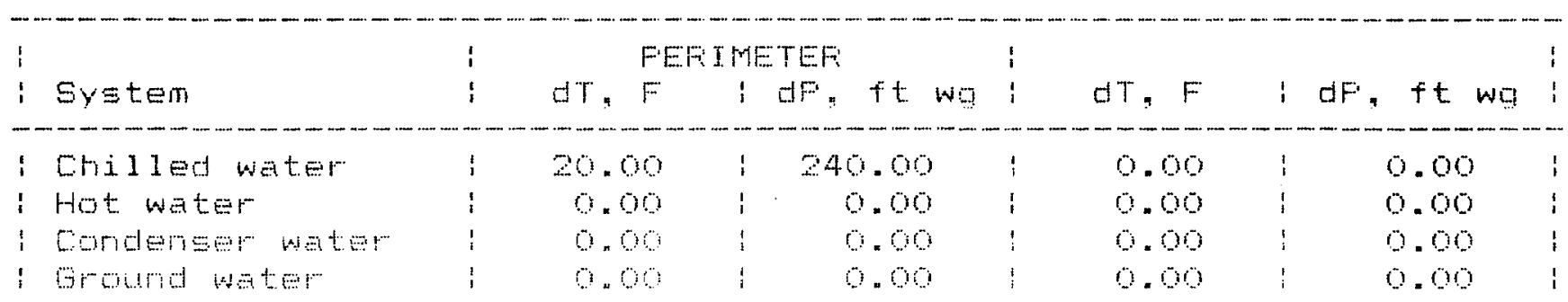

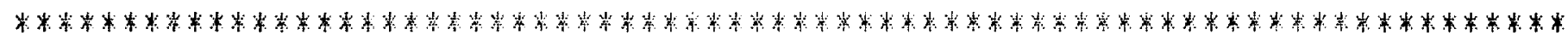

E. MON w

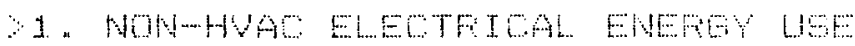

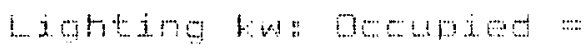

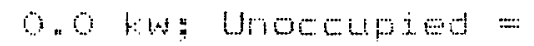
$0.0 \quad 40$

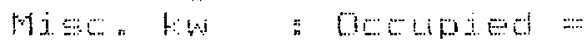
600.
o twa Uromoind
20004
other tin

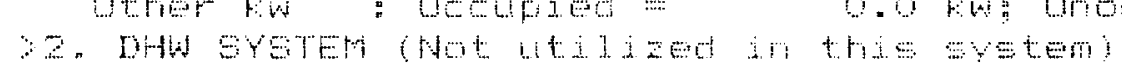

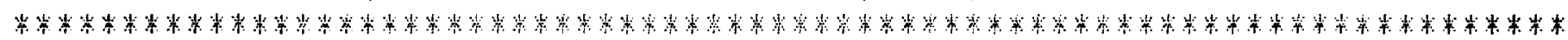

F. FIEI COSTE:

\begin{tabular}{|c|c|c|c|c|c|c|c|}
\hline$\therefore$ & ! & \multicolumn{2}{|c|}{$F=r i o d$} & $i$ & $\because$ ambr Fumis & $\dot{i}$ & Wret $\mathrm{Co}=\mathrm{t}$ \\
\hline ELLETHILIL & 1 & acsise & : Umon & i & n & (n) & 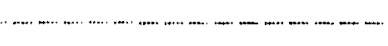 \\
\hline ENFG USE & $i$ & $($ otanis) & $(t / 6)$ & $\vdots$ & Weturas $6=$ & $\vdots$ & $n$ restren \\
\hline - & & & & 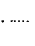 & ifuel Oil & $!$ & $\$ 4.9 .9 \mathrm{a}$ \\
\hline Wrmpres $=0$ & $i$ & 0.0 & 3. 00 & ; & Fimarim & $\vdots$ & $61 \mathrm{~b}$ \\
\hline 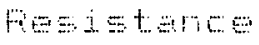 & $!$ & क. क्ष & 6. & $\vdots$ & I remets Hewtsma & $!$ & ANIET! \\
\hline $\operatorname{lndtots} \theta$ & 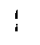 & " & ब & $\vdots$ & Fisnots mol ing & $!$ & G \\
\hline
\end{tabular}




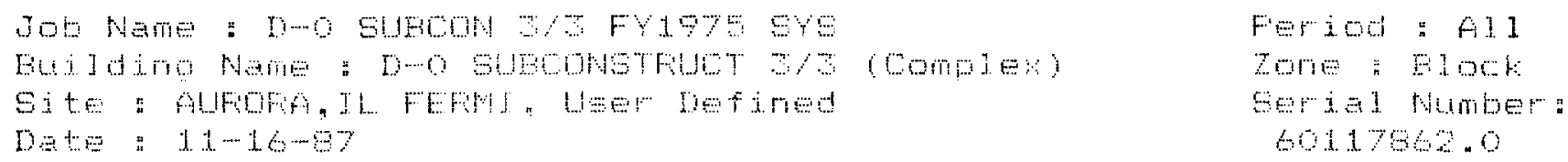

Hey:

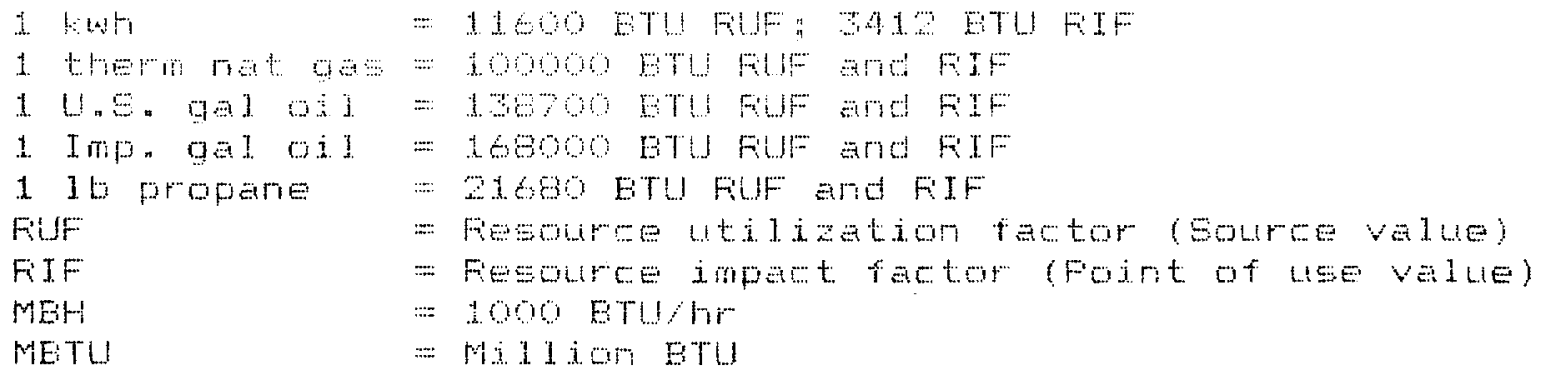




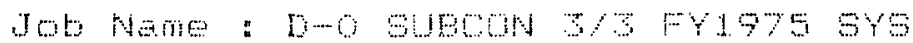

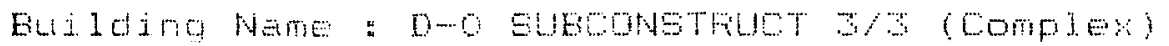

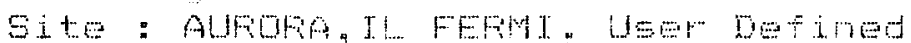

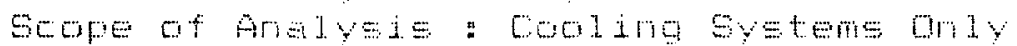

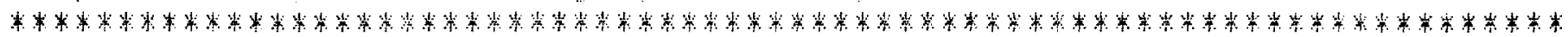

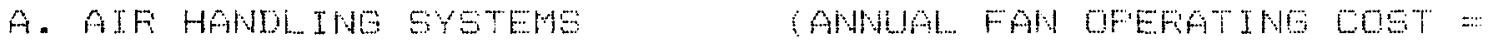

क 40

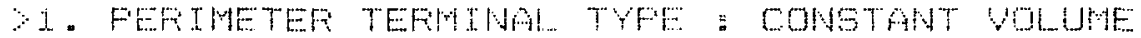

Supply fan type m Forkard mured

Supply fan total static pressure $=0.01$ in. wg.

A returm far is not LSEd.

Design Supply air= $50315 \mathrm{cfm}$. $55 \mathrm{FqVent.} \mathrm{air=} \mathrm{cfm}$

Are cooling terminals used for heating ? ry

Ari ecoriomizer is riot lised.

Is a ventilation reclain devioe used ? r.

Are ventilation air dampere closed for unoceupied periods?

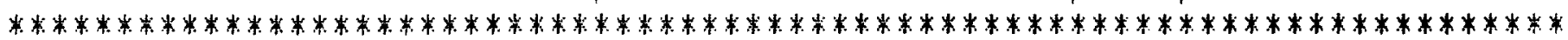

E. HEATING FLANTS (ANNUAL HEATING FLANT OFEFATING CDST =

\% 1 . FEFIMETEF FLANT " EIECTFIOAL FESISTANCE

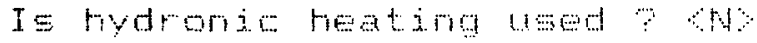

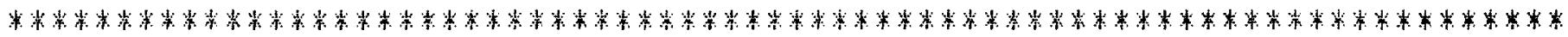




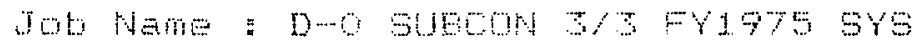

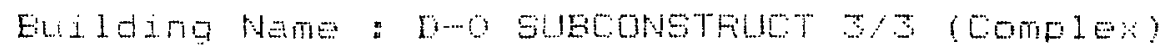

Fage 2

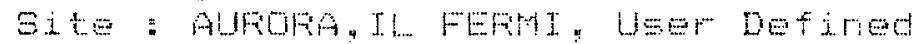

Dats: $11-16-87$

60117662.0

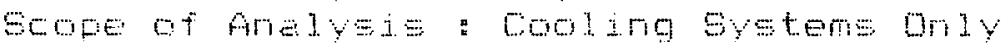

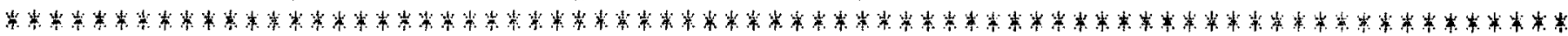

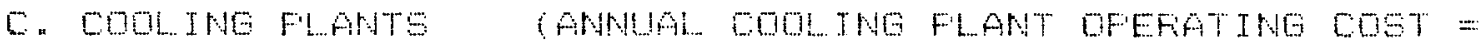

\$1)

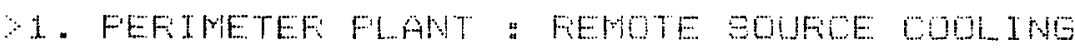

IE Mydronic coo!ing used ? Y

Is chilled water reset $45 e d ?$ No

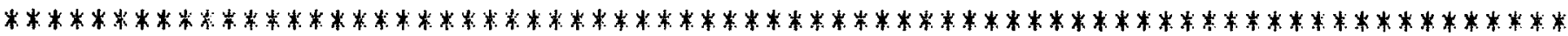

D. FUNFING SYSTENG

(ANNUAL FUNF DFEFATINO COST =

$\$ 5,: 00)$

\begin{tabular}{|c|c|c|c|c|c|c|c|c|}
\hline & $i$ & $\mathrm{PH}$ & mo & TEF & ! & & & \\
\hline Systeni & $i$ & $d T, F$ & 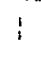 & dF, ft wg & $i$ & $d T, F$ & $d F:$ & $f t$ \\
\hline Chil1ed ajater & $i$ & 20.00 & 1 & 240.00 & $i$ & 0.00 & $!$ & 0.00 \\
\hline Hot water & $i$ & 0.00 & $i$ & 0.00 & 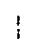 & 0.00 & $\vdots$ & 0.00 \\
\hline Conderiser hater & $i$ & $9 \times 9$ & $!$ & 0.0 & $i$ & 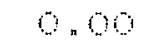 & 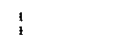 & 0.00 \\
\hline Gmound hater & $\vdots$ & $\theta_{n} 0$ & 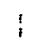 & 9.0 & $i$ & 0,00 & $i$ & 0.00 \\
\hline
\end{tabular}

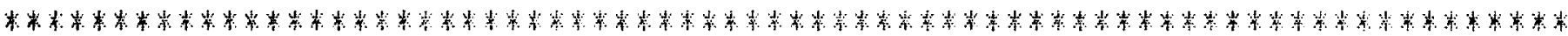

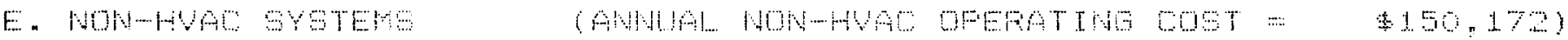

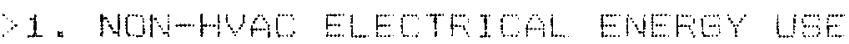

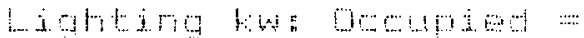

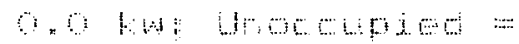
o

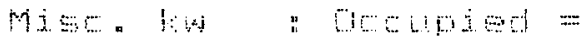

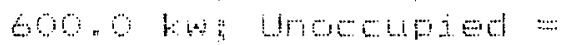
$20 \%$ i
$0+1 \%$ \%

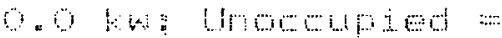
i 12,4

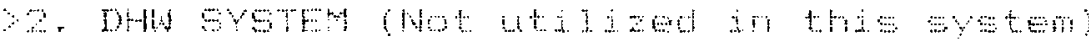

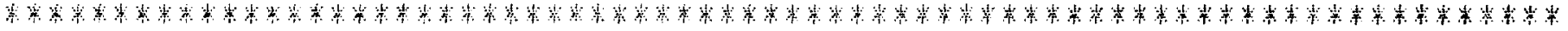

F. FUEL COST

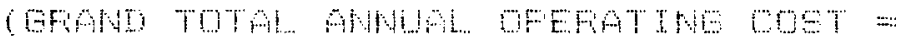

क 15,5201

\begin{tabular}{|c|c|c|c|c|c|c|c|c|}
\hline $1 n$ & $\vdots$ & \multicolumn{3}{|c|}{ Feirod } & 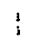 & Z OTHEF FUELS & 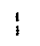 & indt $\quad[0=1$ \\
\hline ELEOTFIAO & $\vdots$ & का & $\frac{1}{i}$ & Whom & & & & \\
\hline EHEWH & $i$ & (A) & $\vdots$ & (Atanis: & $i$ & INatarat 6 & i & $n=2 / t \operatorname{sen}$ \\
\hline 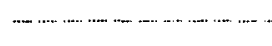 & 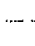 & 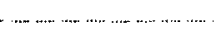 & 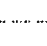 & 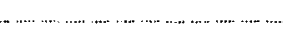 & ......... & Hul oil & 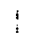 & $\$ 10.6,0$ \\
\hline anprestom & $\vdots$ & 0.06 & $i$ & 0 " & 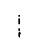 & iromene & $\vdots$ & $\pm 1 \mathrm{~b}$ \\
\hline 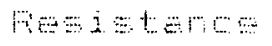 & 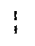 & ॠ & 1 & ; а & $\vdots$ & remote Hestro & $\vdots$ & stmil \\
\hline $19+6+j y$ & $\vdots$ & . & $\vdots$ & 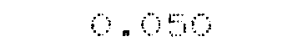 & $\vdots$ & Hemote Coolino & 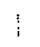 & ब.ली अ \\
\hline
\end{tabular}




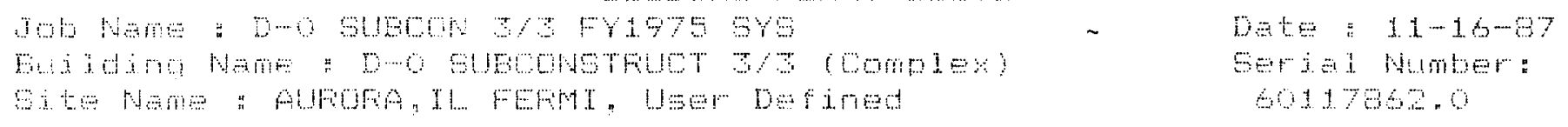

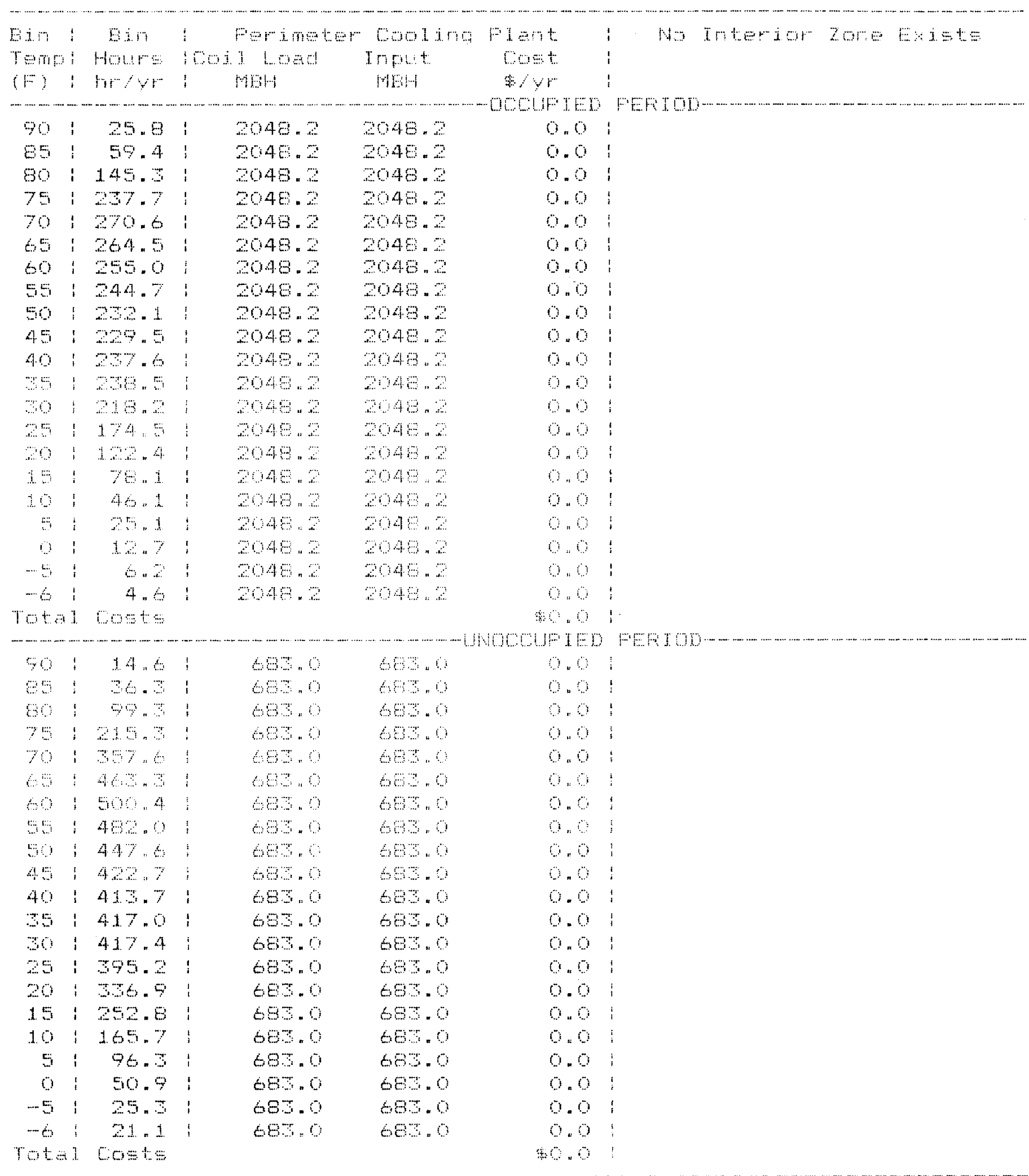




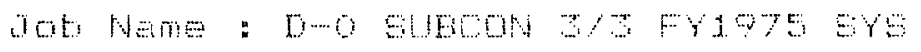

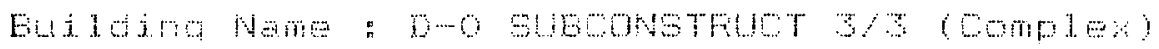

Dete: : $11-16-87$

Serael Nimber: 6117922.0

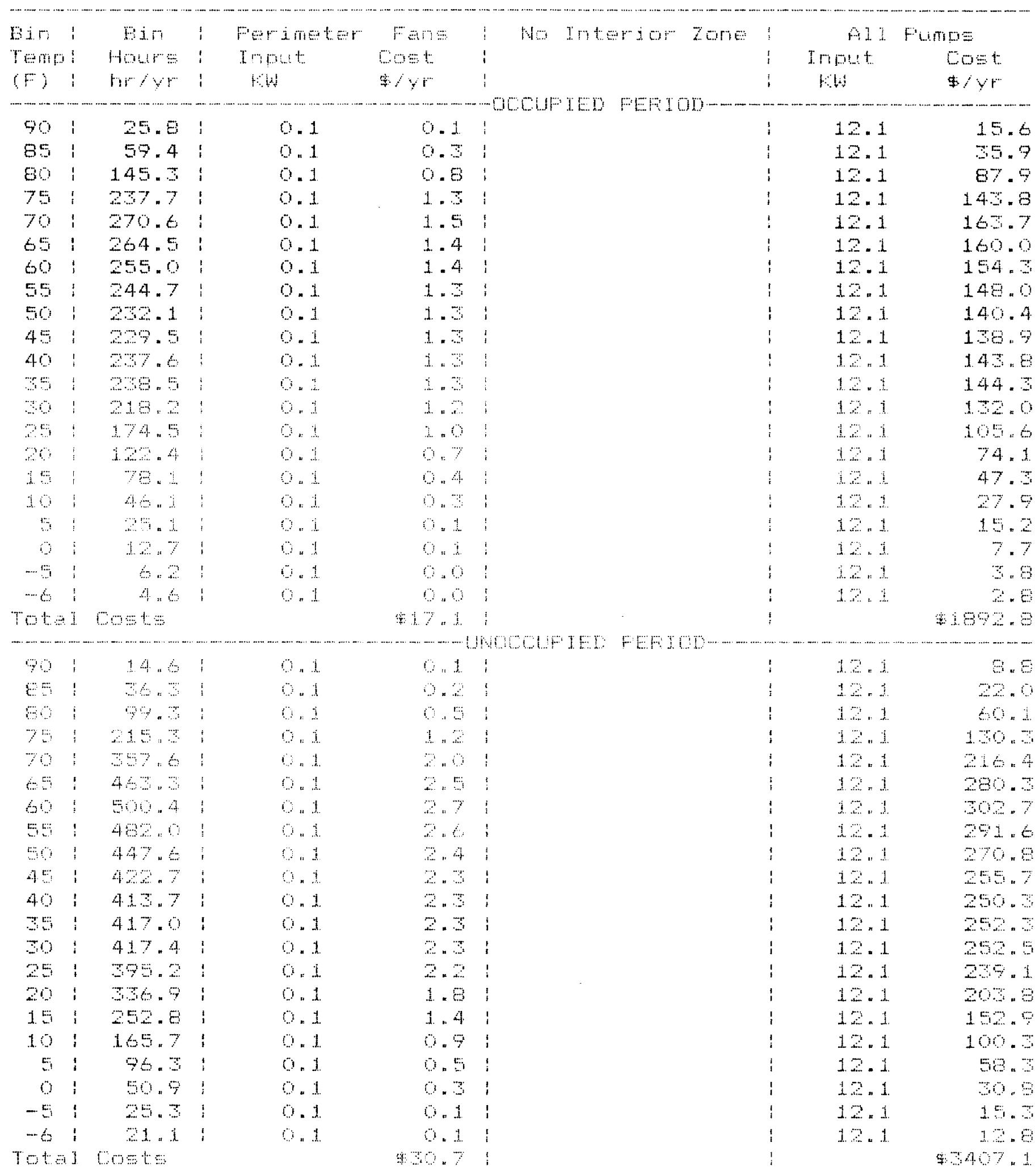




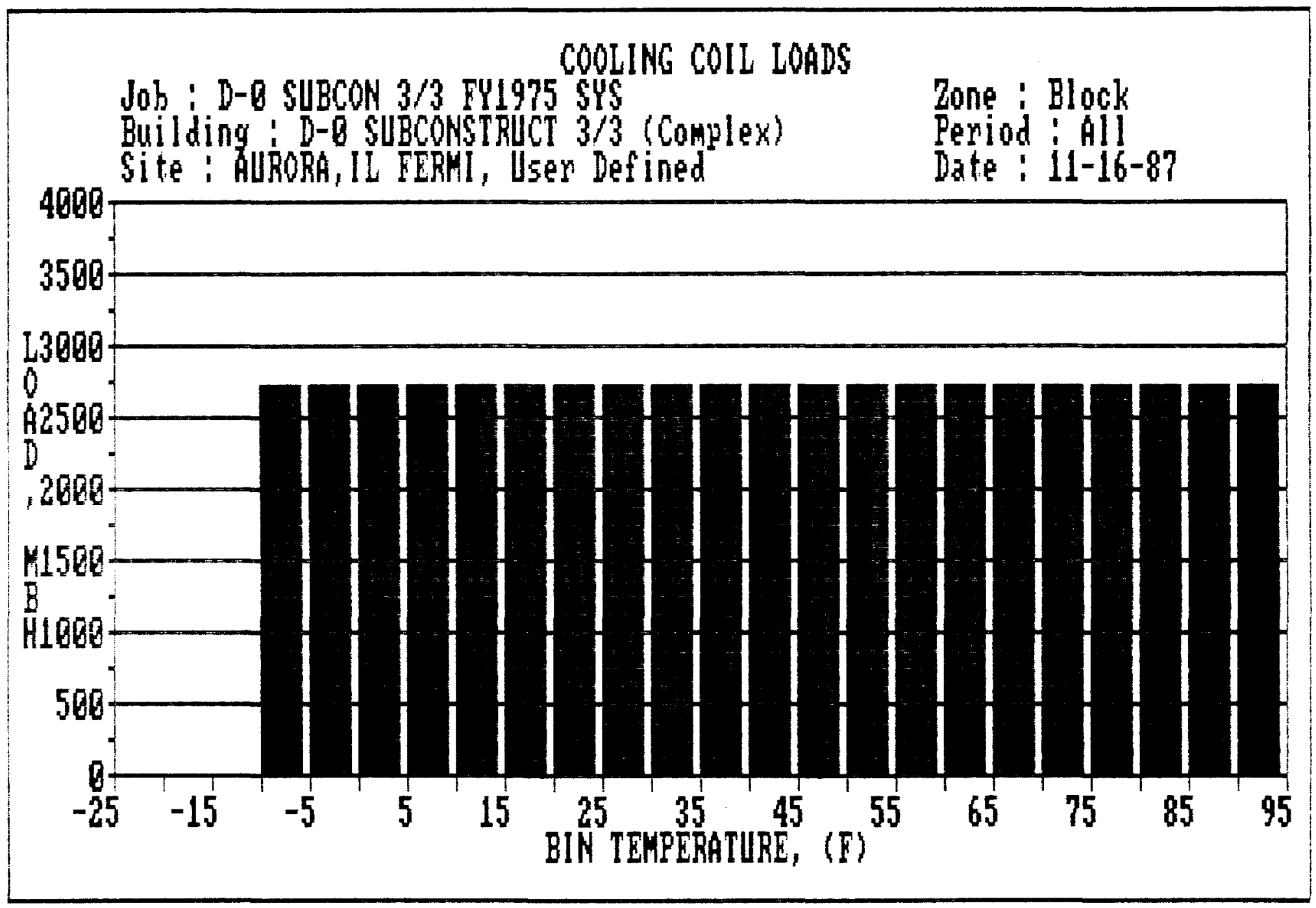


VII.

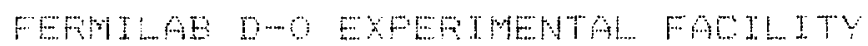

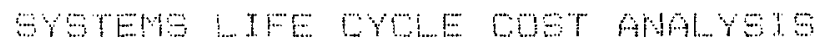

AMD

FABAC CALUHATIOHS

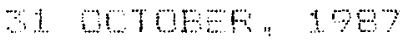

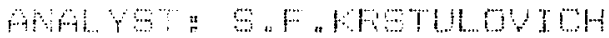

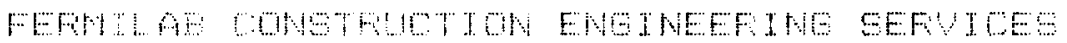




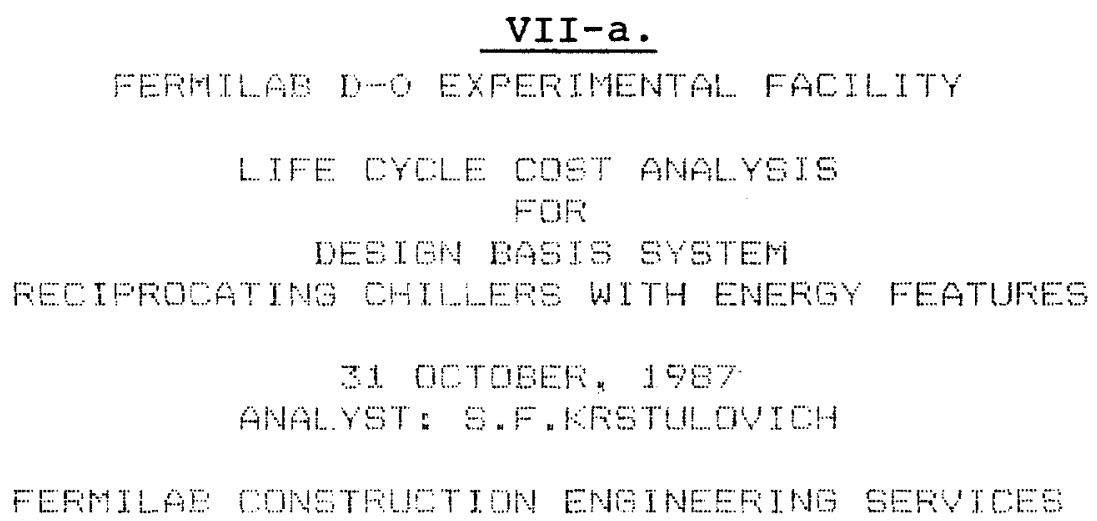


FFOJECT NAME

ANALYST

COMINENTS

ESTIMATE YEAR

COMISESTON YEAF

LAST STUDY YEAF
"FEAMIIAE DO EXFEFTMENTAL FACILITY

: E.F. . FSTLLOUCH

" DESTON BASTS SYSTEM

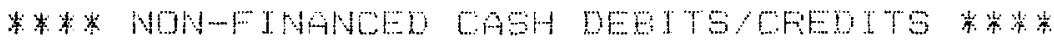

YEAF AMOUNT

1987752900.00
\end{abstract}

**a* FINANCE INSTFLMENTS *****

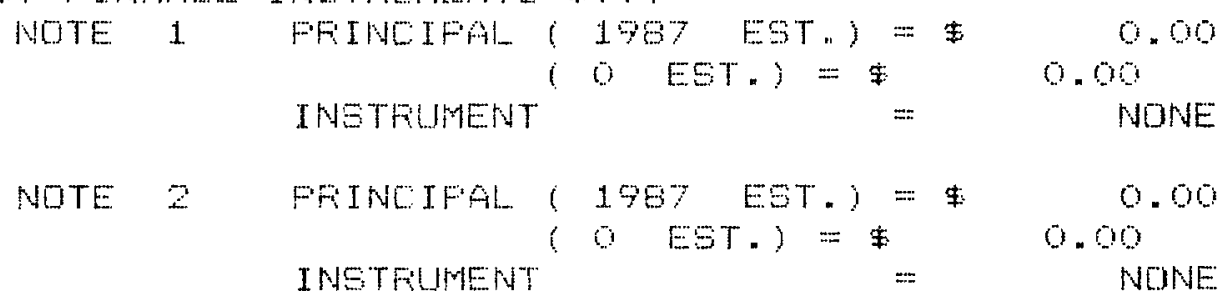

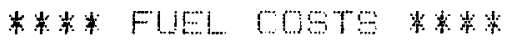

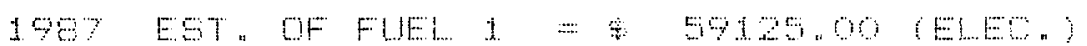

$\begin{array}{lll}2=0 & 00 \\ z=\% & 000\end{array}$

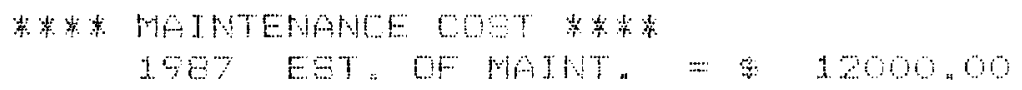

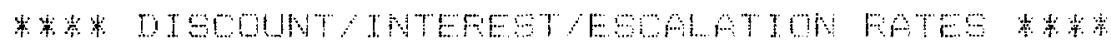

\begin{tabular}{|c|c|c|c|c|c|c|c|}
\hline \multirow[b]{2}{*}{$Y E A F$} & \multirow{2}{*}{ DIs } & \multicolumn{2}{|c|}{ FINANCE ${ }^{n_{n}}$} & \multicolumn{4}{|c|}{ 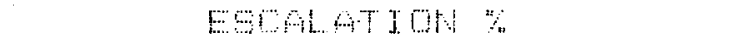 } \\
\hline & & WOH & 1012 & FUEL I & FUE & FUE & Man. \\
\hline 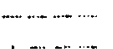 & $+\cdots \cdots+\cdots$ & 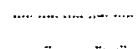 & . & ……………......... & 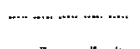 & ……..................... & $-3-1-\cdots$ \\
\hline 1907 & 0.60 & 0.0 & 0.00 & 0.00 & 0.00 & 0.0 & .00 \\
\hline 199 & 7.90 & ब, & .00 & . . 40 & \% & , & 7.00 \\
\hline 1909 & 7.9 & .0 & on & 8.6 & 0.00 & 0.90 & 7.00 \\
\hline 1990 & 7.90 & a & 0.0 & 8.60 & . & 0.00 & 7.00 \\
\hline 1901 & 7.90 & .00 & 0.00 & 8,80 & 0.0 & 0.0 & 7.00 \\
\hline 1992 & 7.90 & a. & 00 & 8.80 & 0,00 & 0 & 7.00 \\
\hline 199 & 7.90 & , & 0.0 & 8,80 & 00 & 0.0 & 7.0 \\
\hline 1994 & 7.90 & 0.0 & 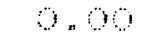 & 8.80 & 0.0 & $=00$ & 7.0 \\
\hline 1995 & 7.90 & 0.00 & .00 & 8.00 & 0.00 & 0.00 & 7.00 \\
\hline 1996 & 7.90 & 0.00 & 0.00 & 8.80 & 0.00 & 0.00 & 7.00 \\
\hline 1997 & 7.90 & 0.00 & 0.00 & 8.80 & 0.00 & 0.00 & 7.00 \\
\hline 1998 & 7.90 & 0.00 & 0.00 & 8.80 & 0.00 & 0.00 & 7.00 \\
\hline 1999 & 7.90 & 0.00 & 0.00 & 8.80 & 0.00 & 0.00 & 7.00 \\
\hline 2000 & 7.90 & 0.00 & 0.0 & 0.80 & 0.00 & 0.00 & 7.00 \\
\hline 2001 & 7.90 & 0.00 & 0.00 & 8.90 & 0.00 & 0.00 & 7.00 \\
\hline 2002 & 7.90 & 0.00 & 0.00 & 8.80 & 0.00 & 0.00 & 7.00 \\
\hline 2005 & 7.90 & 0.00 & 0.00 & 8.80 & 0.00 & 0.00 & 7.00 \\
\hline 2004 & 7.90 & 0.00 & 0.00 & 8.80 & 0.00 & 0.00 & 7.00 \\
\hline 2005 & 7.90 & 0.0 & .00 & 8.80 & 0.00 & 0.00 & 7.00 \\
\hline 200 & 7.90 & $\mathrm{O} . \mathrm{o}$ & 0.0 & 8.60 & 0.0 & 0.00 & 7.00 \\
\hline $20 \%$ & 7.9 & o. & , & $\mathrm{e}$ & का & 0.0 & 7.00 \\
\hline 208 & 7.90 & 0 & ०. & 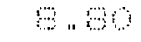 & बा & . & 7.00 \\
\hline
\end{tabular}




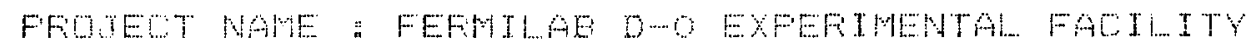

AHALYT MAHE "

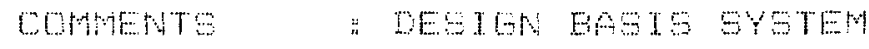

\begin{tabular}{|c|c|c|c|c|c|c|}
\hline \multicolumn{2}{|c|}{$Y$ rar } & 1907 & 190 & $196 \%$ & $1 \%$ & 1951 \\
\hline 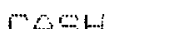 & $\therefore$ & $\gamma[-\cdots, \infty, \infty$ & 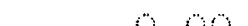 & 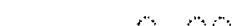 & 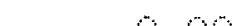 & 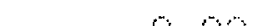 \\
\hline & & 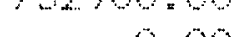 & 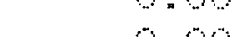 & $\therefore$ & 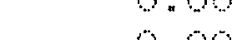 & $\because=4$ \\
\hline MOFT $=1$ & 平 & 0.010 & 0.0 & 0.0 & 0.04 & 0,0 \\
\hline MUFT. 2 & \$ & 0.00 & 0.00 & 0.00 & 0.00 & 0.00 \\
\hline FUEL 1 & $\$$ & 0.00 & 59490.98 & 59694.43 & 60394.02 & 60897.77 \\
\hline FUEL 2 & $\$$ & 0.00 & 0.00 & 0.00 & 0.00 & 0.00 \\
\hline FUEL 3 & \$ & 0.00 & 0.00 & 0.00 & 0.00 & 0.00 \\
\hline MATNT. & $\$$ & 0.00 & 11899.91 & 11800.65 & 11702.22 & 11604.61 \\
\hline & & $-\cdots--\cdots-\cdots$ & 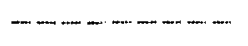 & - & $-1-\cdots$ & 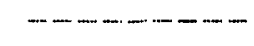 \\
\hline YEAF: & 击 & 752900.00 & 71290.89 & 71695.09 & 72096.27 & 72502.56 \\
\hline & & - & - & 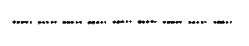 & $-\cdots---\cdots$ & 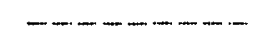 \\
\hline & & 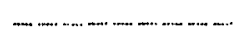 & 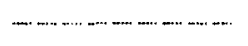 & (n) & 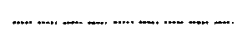 & $---m-m-n$ \\
\hline EUt. & 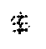 & $7=99000$ & 62429.90 & 9959- . & 9679919 & 1040492 - \\
\hline
\end{tabular}

\begin{tabular}{|c|c|c|c|c|c|c|}
\hline \multicolumn{2}{|l|}{ YEAt? } & 199 & 199 & 199 & 1995 & 199 \\
\hline $\mathrm{CAs}$ & $\%$ & $1=0$ & 0.0 & 0,0 & $0{ }_{n}$ & ओ. \\
\hline MOF" & $*$ & ओ & ॥ बो & "० & 0.0 & 0,0 \\
\hline MOFT & $\$$ & बओ & ओ & ओं & i) & का \\
\hline FUE 1 & $\dot{\xi}$ & $6140 \%$ & ब1 97.9 & $24,4,3$ & 69619.8 & 6340,27 \\
\hline$F \cup E 1 \quad 2$ & $\dot{q}$ & बण & . & . & i & o. \\
\hline FUEL & $\dot{\psi}$ & 9.0 & . & \% & 9.00 & 0.00 \\
\hline MATNT: & i & 115070 & 114110 & $1 \pm 1 \pm .6$ & $1122-2$ & 11128.6 \\
\hline & & $-\cdots$ & 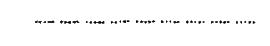 & 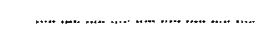 & 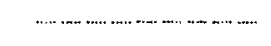 & -1 \\
\hline YEAS & 4 & 7291054 & $79 \%, 76$ & 7751.02 & 74177.41 & $7400: 91$ \\
\hline & & 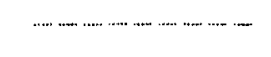 & 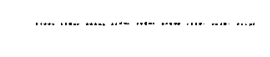 & ……n- & … & $-\ldots+\ldots$ \\
\hline & & $+7=$ & 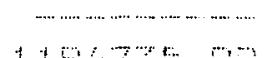 & (n) & 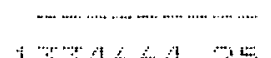 & 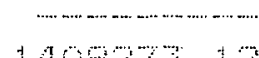 \\
\hline but. & 7 & 1113400 , & 110736 & 1264060 & 16464,25 & $1409,3,12$ \\
\hline YEAF & & 199 & 199 & 199 & शीणी & 201 \\
\hline & & $-\cdots-\cdots-\cdots$ & 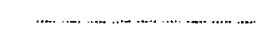 & 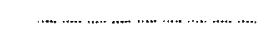 & … & (n- \\
\hline mon & 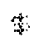 & $\mathrm{a}, \mathrm{ll}$ & " & ओ & $\log _{n}$ & "ळ": \\
\hline MOFT " 1 & 4 & 0,0 & in & $\mathrm{n}_{40}$ & $0 \cdot 10$ & अ \\
\hline Müग & 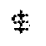 & 0.00 & o. & \% & 0.00 & 0.00 \\
\hline$F l(\ldots i \ldots 1$ & $\$$ & 64009.70 & 64543.67 & 65092.04 & 65624.89 & 60172.27 \\
\hline 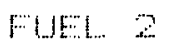 & \$ & 0.00 & 0.00 & 0.00 & 0.00 & 0.00 \\
\hline FUEL & 中 & 0.00 & o. & ब & 0.00 & 0.00 \\
\hline MAINT. & 事 & 1105.82 & 1004377 & 1095.49 & 10761.97 & 10672.20 \\
\hline & & $---m-n-1-n$ & 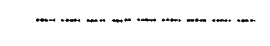 & $-\cdots$ & 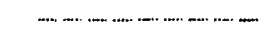 & $---1---1$ \\
\hline YEAF $S$ & 生 & 75045.58 & 75467.44 & 7994.52 & 769686 & 76844.47 \\
\hline & & 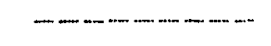 & 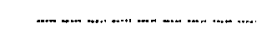 & 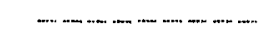 & $-\cdots---m---$ & 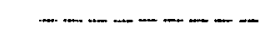 \\
\hline & & 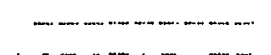 & 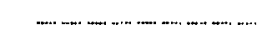 & -3 & 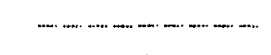 & $-----1--1$ \\
\hline CuM. & 本 & 1484.18 .75 & 1559606 - & 1635740,75 & 1712127,62 & 1789972.12 \\
\hline
\end{tabular}




\begin{tabular}{|c|c|c|c|c|c|c|}
\hline \multicolumn{2}{|c|}{ YEAP } & 2002 & $20 \mathrm{z}$ & 2004 & 200 & 2006 \\
\hline $\mathrm{CASH}$ & $\$$ & . & ") & . & 0.00 & 0.00 \\
\hline$r=1$ & 3 & o. & . & $i_{n}$ & . & 0.00 \\
\hline$T \cdot 2$ & 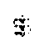 & $m_{n}$ & ${ }_{n} \mathrm{Oo}$ & o. & on & 0.0 \\
\hline FUIEL 1 & & 66724. & 67200.77 & 67841.9 & 69407.94 & 69978.43 \\
\hline$F \cup F=$ & S & 0 & 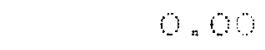 & & .00 & 0.00 \\
\hline FUEL $\quad:$ & 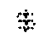 & $.0 \%$ &. & 0 & .00 & 0 \\
\hline MATHT & s & 10505.19 & 10494.91 & $3040 \%, 37$ & 1082,56 & 10294.40 \\
\hline & & $---\cdots---$ & $-\cdots---$ & $-\infty-\infty-1-m$ & $-\cdots-\cdots$ & $--m-\cdots$ \\
\hline YEAF'S & 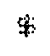 & $77307 \cdot 41$ & 77775.60 & 78249.3 & 78728.40 & 79212.91 \\
\hline & & $-1-0$ & $---1-1$ & $-1-5-1$ & $-1-0-1$ & \\
\hline (llk. & 来 & 1866279,50 & 1944055.12 & 2022304.50 & $21010 \mathrm{~g} .00$ & 2190246.00 \\
\hline
\end{tabular}

\begin{tabular}{|c|c|c|c|c|c|c|}
\hline \multicolumn{2}{|c|}{ YEAF: } & 2007 & $200 \theta$ & 2009 & 2010 & 2011 \\
\hline ФАН & 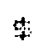 & 0.00 & . 00 & 0.00 & 0.00 & 0.00 \\
\hline$T .1$ & \% & 0.00 & ब, & 0.0 & 0.00 & 0.00 \\
\hline 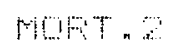 & : & 0,0 & ओ & \% & 0.0 & " \\
\hline mein & 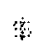 & $60,6 \%, 79$ & 70,94 & .00 & त. & 0.00 \\
\hline FuW $=$ & s. & 0,0 & o. & , a & 0.60 & 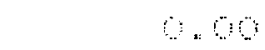 \\
\hline FUE & : & o. & 90 & , क & 1.00 & $n_{n}$ \\
\hline MAXU" & s & $1014 \%, 21$ & 100,46 & 0.0 & . बो & .60 \\
\hline XEA & $s$ & 79709 & 90196,8 & .00 & , & . \\
\hline & & 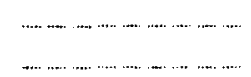 & 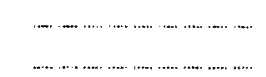 & 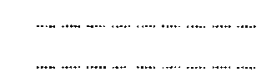 & 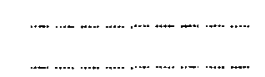 & (n) \\
\hline$\%$ & & 21979,00 & 2340147.60 & 2340147,50 & $2340,47.50$ & 2840147.50 \\
\hline
\end{tabular}




\author{
VII-b. \\ FEFMTLE DOO EXFERMENAL FACILTY \\ LIFE GYLE COST ANALYSS \\ For? \\ ALTENATE OOMFATISOH SYSTEM NUMEEF I

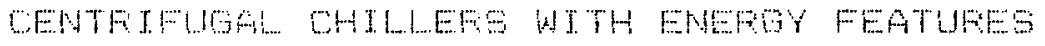 \\ 3 OOTOEF $198 \%$

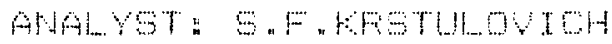

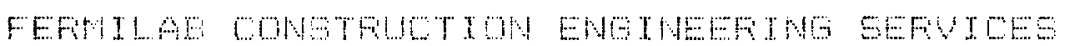


PFOJEET NAHE AIALYST

COHHENTS

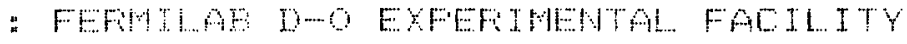

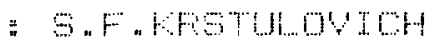

: AL TENATE SYSTEN NO. 1

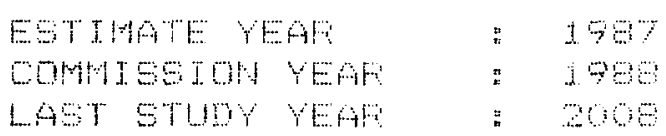

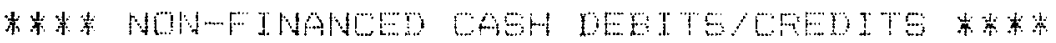

YEAF AMOUNT

1987752900.00

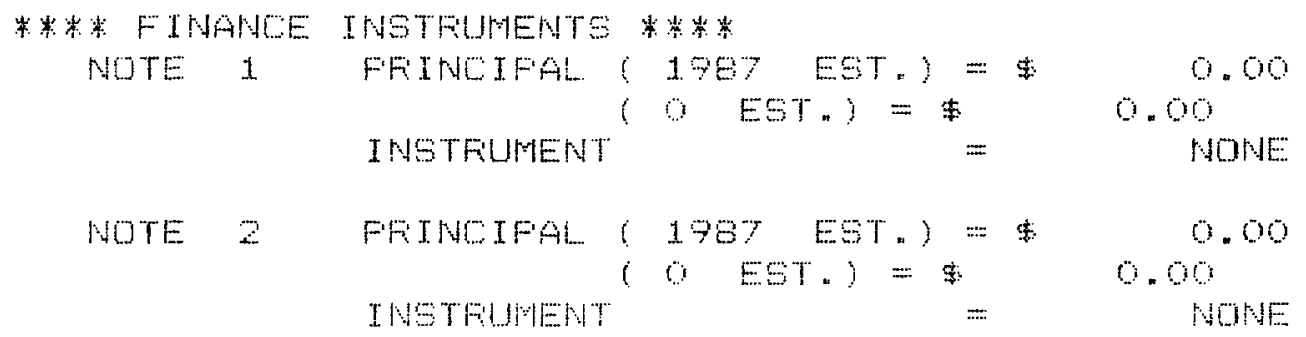

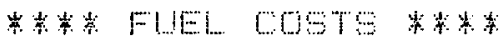

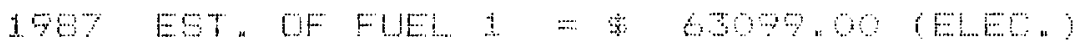

$\begin{array}{lll}z=8 & 00 \\ 3=\% & 0.00\end{array}$

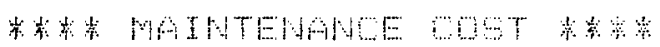

1967 EST "OF MAINT = 12000 "OO

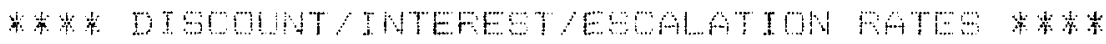

\begin{tabular}{|c|c|c|c|c|c|c|c|}
\hline & & $F I N$ & $E$ & & EDAL & In $\%$ & \\
\hline YEA & $\operatorname{siscon}$ & LAfH & LDAnL & FUE & FUEL2 & FUEL & Hata. \\
\hline & 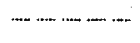 & 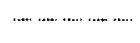 & 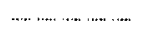 & 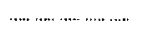 & 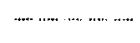 & $-\ldots-\ldots$ & 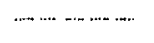 \\
\hline 1987 & 0.00 & . & 0.00 & ") " & 0.00 & .00 & $\pi$ \\
\hline 1990 & 7.90 & $0, \infty$ & 0.00 & 8.40 & 0.00 & $\mathrm{or}$ & 7 \\
\hline 196 & 7.90 & an & 0.00 & 8.60 & 0.00 & .00 & 7.00 \\
\hline 1970 & 7.90 & $\mathrm{an}$ & 0.0 & e. & 0.00 & $\mathrm{OH}$ & 7.0 \\
\hline 1991 & $7 \times 90$ & 0.0 & 0,0 & $e .8$ & 0.0 & 0.00 & 7.00 \\
\hline 199 & 7.90 & . & 0,0 & 8.8 & 0.00 & . & 7.00 \\
\hline 199 & 7.9 & ${ }_{n}$ & \% & $:, 6$ & 00 & . & 7.00 \\
\hline 1994 & 7.90 & ॠ. & 0.00 & 8.00 & 0,00 & 0.00 & 7.00 \\
\hline 1995 & 7.90 & o. & 0.00 & 8.90 & 0.00 & 0.00 & 7.00 \\
\hline 1996 & 7.90 & 0.00 & 0,0 & 8.80 & 00 & 0.00 & 7.00 \\
\hline 1997 & 7.90 & 0,00 & 0.0 & 8.80 & . & 0.00 & 7.00 \\
\hline 1998 & 7.90 & 0.00 & 0.00 & 9.60 & 0.00 & 0.00 & 7.00 \\
\hline 1999 & 7.90 & 0.00 & 0 & 8.80 & 0.0 & 0.00 & 7.00 \\
\hline 2000 & 7.90 & 0.0 & 0,00 & 8.60 & 00 & 0.00 & 7.00 \\
\hline 01 & 7.90 & 0.00 & 0.0 & 8.80 & 0.00 & 0.00 & 7.00 \\
\hline 20 & 7.90 & 0.00 & 0,0 & 8.80 & 0.00 & 0.00 & 7.00 \\
\hline 2005 & 7.90 & 0.00 & 0.00 & 8.80 & $0.0 \%$ & 0.00 & 7.00 \\
\hline 2004 & 7.90 & 0.0 & 0.0 & 8.80 & 00 & 0.00 & 7.00 \\
\hline 205 & 790 & 0.0 & . & 8.80 & अ & 0.0 & 7.0 \\
\hline 200 & 7.90 & . & 0.0 & $\theta .90$ & 00 & \% & 7.0 \\
\hline $20 \%$ & 7.90 & 0.0 & 0.0 & e. & 0.0 & "० & $7 \cdot 0$ \\
\hline 200 & 7.90 & o. & .0 & . & ब. & 0 & 7.96 \\
\hline
\end{tabular}




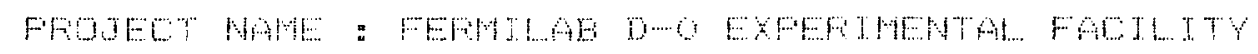

मील YeT NAM :

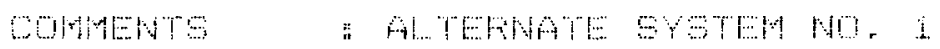

\begin{tabular}{|c|c|c|c|c|c|c|}
\hline \multicolumn{2}{|l|}{$Y E A$} & 196 & 1988 & 1969 & 1990 & 1991 \\
\hline+6 & $\Leftrightarrow$ & $7-990000$ & $\theta_{n}$ & 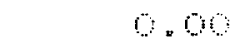 & 0.0 & 9.9 \\
\hline MOFT. I & 来 & 0.00 & 0.00 & 9.0 & 0.00 & 0.00 \\
\hline MUF:T. 2 & 果 & 0.00 & 0.00 & 0.00 & 0.00 & 0.00 \\
\hline FUE 1 & 里 & 0.00 & 6991,9 & 67990.15 & $64453-1$ & 64990.92 \\
\hline FELI. 2 & $\$$ & 9.09 & 0.00 & ${ }_{n} \mathrm{OC}$ & 0.00 & 6.00 \\
\hline FUII & 要 & $\theta, 0$ & 0.00 & 0.09 & 0.00 & 0.00 \\
\hline MAITT. & 专 & 0.0 & 11899.91 & 1100.6 & 11702.2 & 11604.61 \\
\hline & & 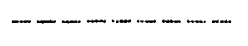 & 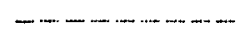 & - & 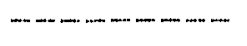 & $-1-1-1-1$ \\
\hline YEA $\mathrm{B}$ & $\$$ & 752900,00 & 79291 . & 757.000 & 76155.52 & 76595.55 \\
\hline & & $-(--1-1--$ & 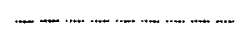 & 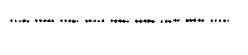 & $\cdots--\cdots$ & 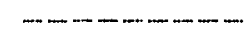 \\
\hline & & (- & 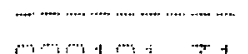 & 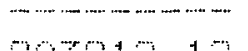 & , & +0 \\
\hline Cunt. & $\$$ & 7590000 & 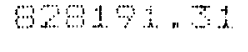 & 9012 : & 900067.62 & $1056665+12$ \\
\hline
\end{tabular}

Yeitio

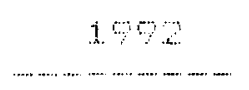

+.

a.t.

का

in

a, का

$0.1 \%$

$6 \sin 00$

का

" $\mathrm{Ol}$

$1+50 \%$

-

$7 \% 40$

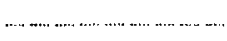

-

Hot.

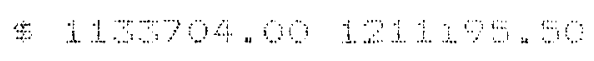

Yw.

\section{L., $1 ;+\cdots$}

Mil,

Mाए?

|........... 1

fill 2

Fin:

MHTT.

$Y E F \div S$

un.
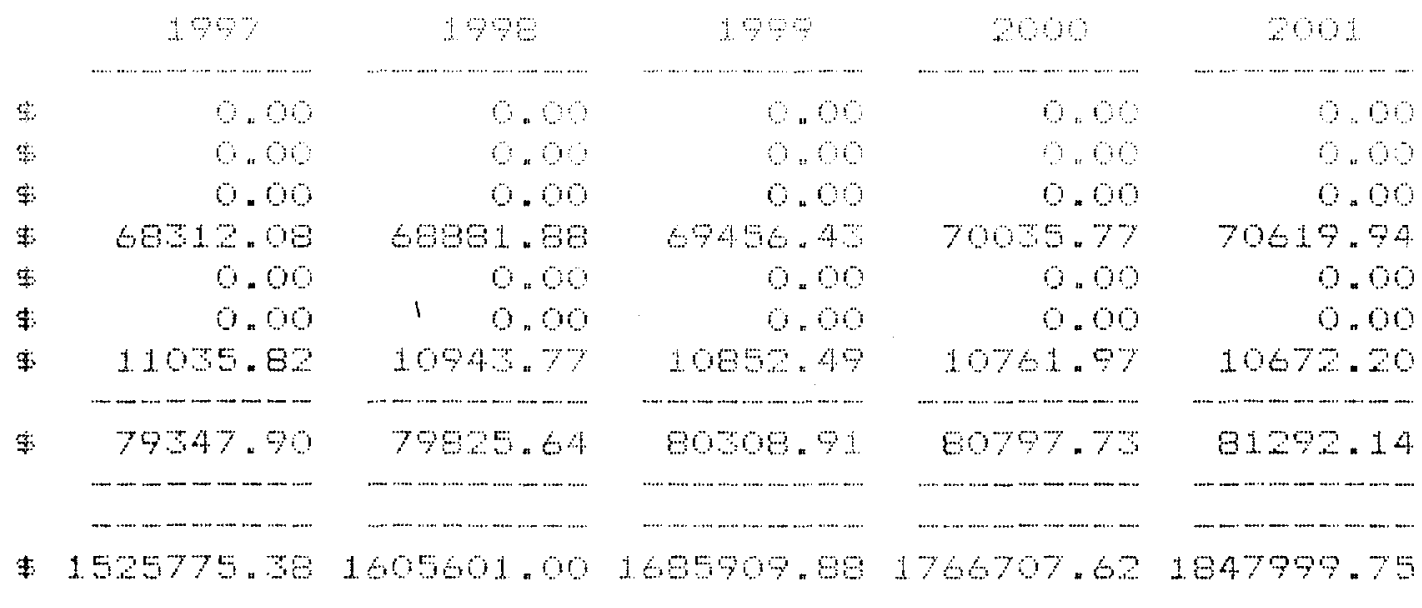

1847999.75

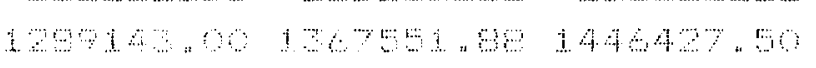
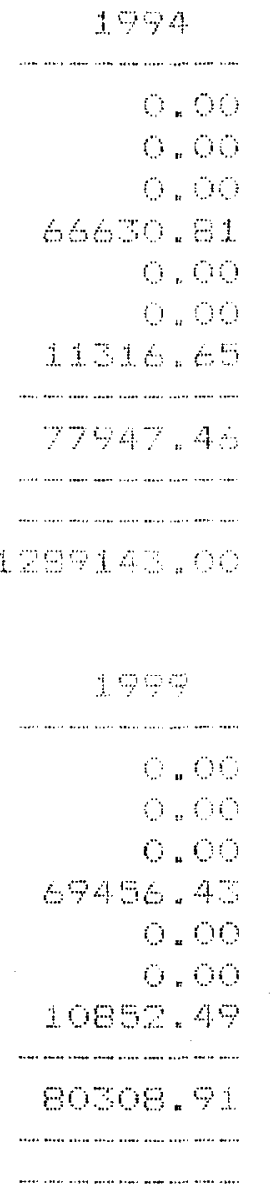

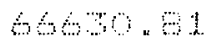

ता

(1)

1 15160

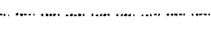

$77947: 46$

ј

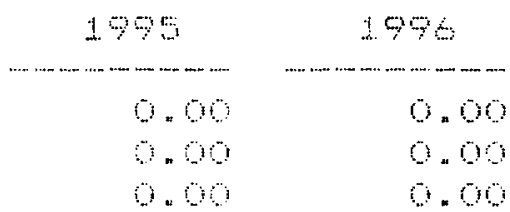

o7 77,00

का

0.0

$1112 \mathrm{~s}$

7907.64

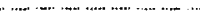

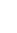

.




\begin{tabular}{|c|c|c|c|c|c|c|}
\hline \multicolumn{2}{|l|}{ YEAF } & 200 & 201 & 2004 & 2005 & 2006 \\
\hline 5,61 & 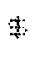 & O० & 00 & क. & का & का \\
\hline More 1 & $\$$ & 1.00 & 0.00 & 00 & .00 & 0.0 \\
\hline MOPT 2 & 3 & 0,0 & 0.0 & 0.00 & 0.00 & 0.00 \\
\hline FLEEL 1 & 7 & 71200.79 & 71002.95 & 72401.67 & 73005.77 & 78614.72 \\
\hline FUEL 2 & $¥$ & $\mathrm{or}_{n}$ & 0.00 & 0.00 & 0.00 & o, \\
\hline FUE! & 7 & .00 & 0.00 & 1,00 & 0.00 & 0.00 \\
\hline MATHT. & $\omega$ & 1056419 & 10494.91 & 10407.37 & 10320,50 & 10234.48 \\
\hline & & 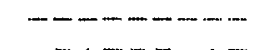 & $---\cdots$ & -- & $-m-n-\cdots$ & $-x-m-1$ \\
\hline YEAF' $S$ & $\$$ & 81792.17 & 82297.67 & 82809.23 & 85326.34 & 83849.20 \\
\hline & & $-1-1-0$ & $-1-1-1-1$ & $-1-1-5-1$ & $-1-2-1-1-1$ & - \\
\hline cut. & $\$$ & 1929791.80 & 2012009.75 & 2094899.00 & 2178225.20 & 2262074.50 \\
\hline YEAF & & 2007 & 2008 & 2009 & 2010 & 2011 \\
\hline CASH & 俦 & 0.00 & 0,00 & 0.00 & 0.00 & 0.00 \\
\hline Mor: - 1 & \pm & 0.00 & 0,0 & 0.00 & 0.0 & 0.00 \\
\hline MOET.2 & t & 0.00 & 0.00 & 0.0 & .00 & 0.00 \\
\hline FuEL 1 & + & 74298.7 & 7494,89 & . & , & 0.0 \\
\hline FUEL $=$ & 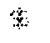 & 0.0 & $0, \mathrm{on}$ & 0.00 & 00 & 0.0 \\
\hline$F \amalg \mathrm{E}$ & 安 & 0.0 & 0 & ब. & "ओ & o. ओ \\
\hline MAINT" & के & 10149 . 11 & 1004 4 46 & का & ब० & मे \\
\hline YEAF" & \% & $94 \% 7$ " & 949120 & , का & की & o \\
\hline & & 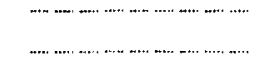 & - & - & (-1) & \\
\hline euti. & \% & $25445=20$ & $2441+64,50$ & 242164,60 & 241264,60 & 243124.60 \\
\hline
\end{tabular}




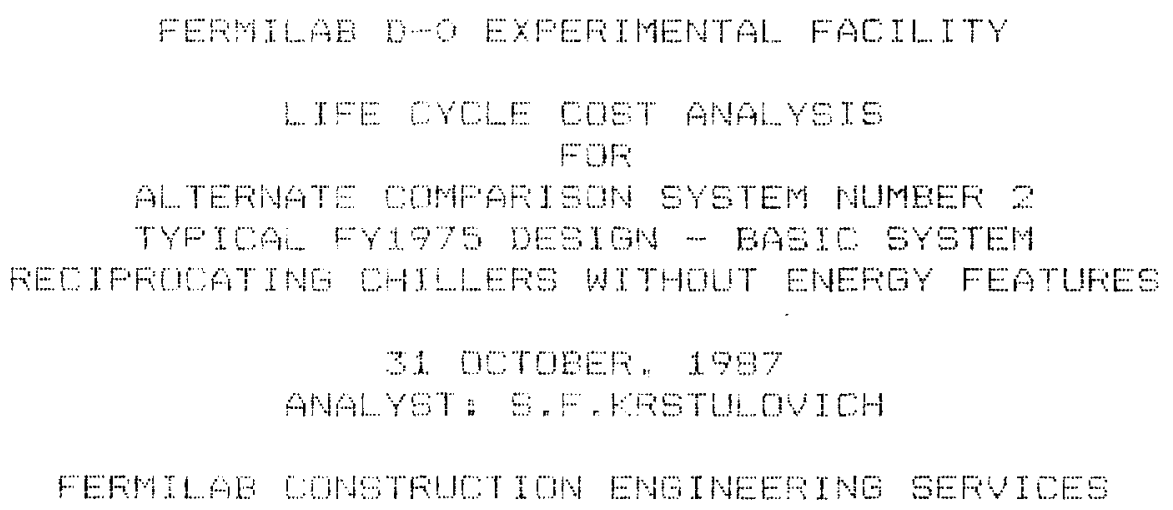


FFPOJEST NAME

ANALY YT

Complans
" FEMULAE OWO EXEETMNTAL FACT TTY

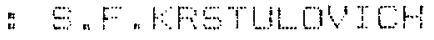

: H. T. SYSTEA NO. 2 - FYमY7 BASIC

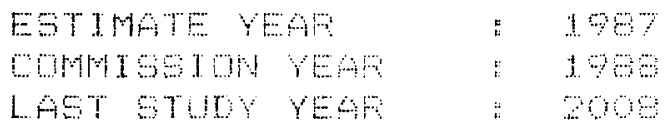

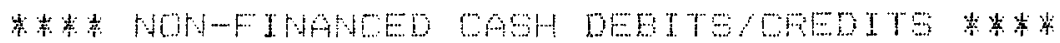

YEAF AHOLNT

1987675400,00

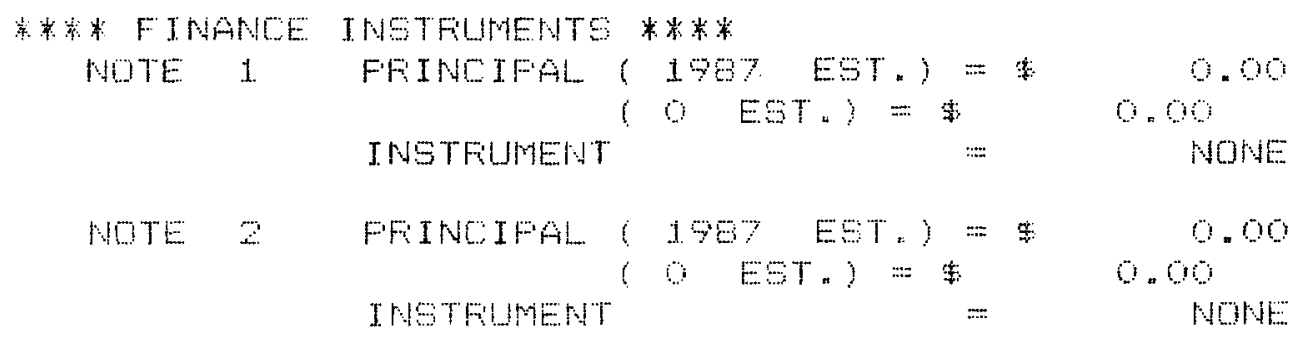

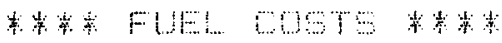

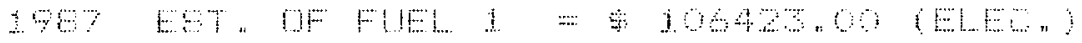

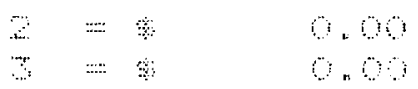

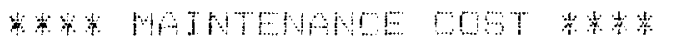

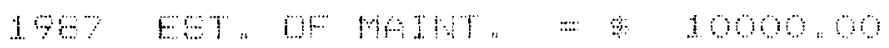

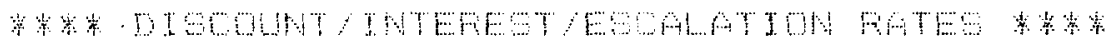

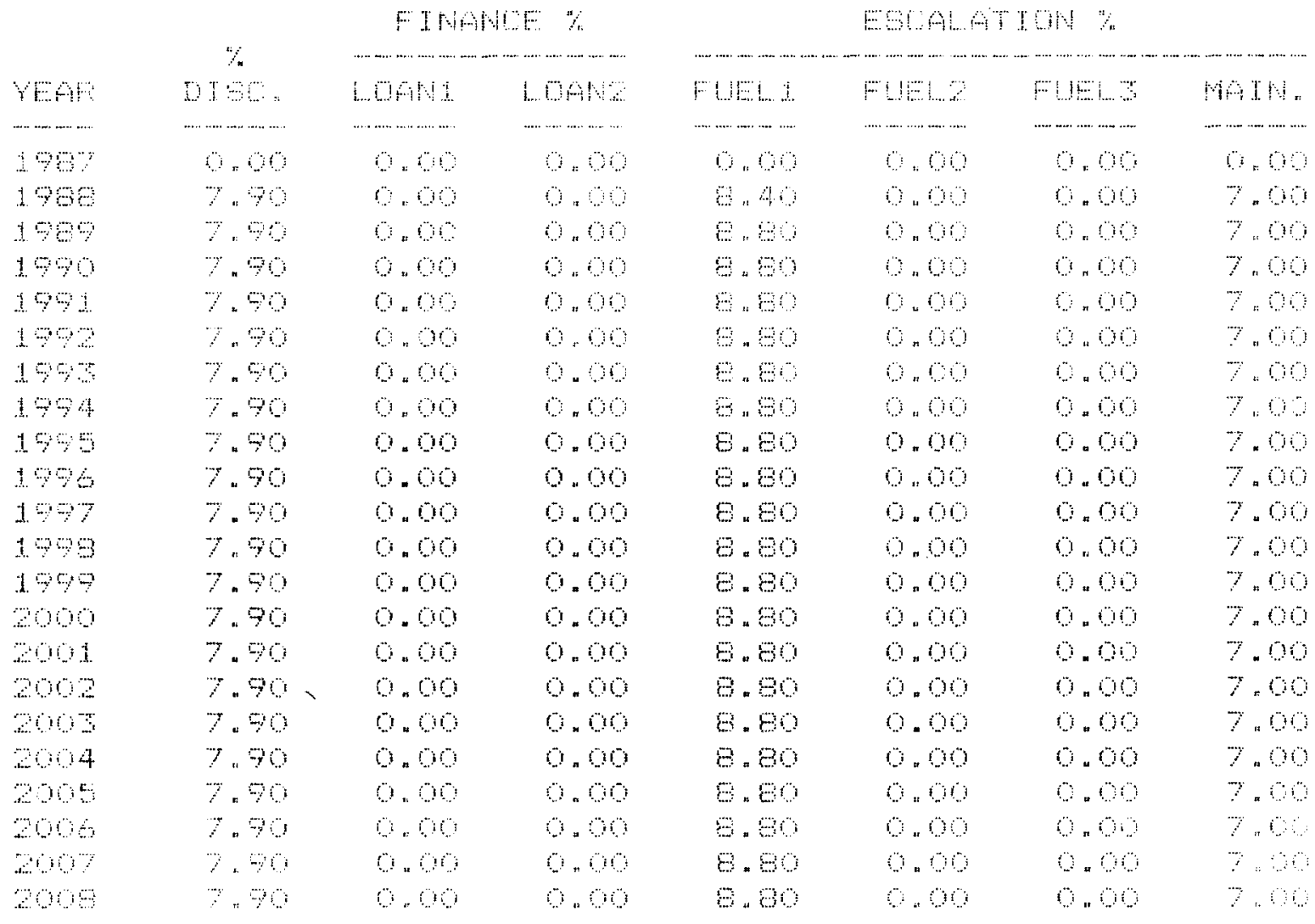


FFOUET NAME : FFMTLA DAO EXPETMENTAL FALILTTY

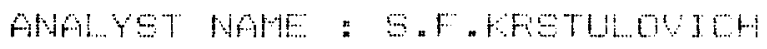
COHAENTS

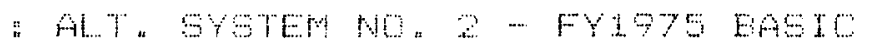

\begin{tabular}{|c|c|c|c|c|c|c|}
\hline \multicolumn{2}{|l|}{$Y E A F$} & $19 \%$ & 196 & 199 & 1990 & 1991 \\
\hline & & $-(-1-1-2$ & (-n) & 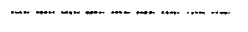 & $-\cdots$ & \\
\hline $\mathrm{CASH}$ & s: & 675400.00 & 0.9 & $\mathrm{O}, \mathrm{O}$ & 0.00 & 0.00 \\
\hline MOFT .1 & $\$$ & 0.00 & 0.00 & 0.00 & 0.00 & 0.00 \\
\hline MOFT. 2 & $\$$ & $0.0 \%$ & 0,10 & 0.00 & 0.00 & 0.00 \\
\hline FUEL 1 & $\$$ & 0.00 & 106916.16 & 107807.95 & 100707.19 & 109613.93 \\
\hline FUUEL 2 & 本 & 0.00 & 0.00 & 0.00 & 0.00 & 0.00 \\
\hline FUEL 3 & 生 & 0.00 & 0.90 & 0.00 & 0.00 & 0.000 \\
\hline \multirow[t]{2}{*}{ MAJNT. } & $\$$ & 0.00 & 9916.59 & 983.80 & 9751.85 & 5670,51 \\
\hline & & $-+-m--m$ & $-(-m-\cdots-\cdots$ & $---\cdots--\cdots$ & --------- & $-\cdots--\cdots-\cdots$ \\
\hline \multirow[t]{3}{*}{ YEAF: $S$} & $\$$ & 675400.00 & 11603274 & 117641.83 & 118459.04 & 119284.45 \\
\hline & & -------- & $-----m-m$ & $---\cdots--\cdots$ & $-------m$ & $--\cdots--m-\cdots$ \\
\hline & & $-m-m-1-\cdots$ & $-3-\cdots$ & 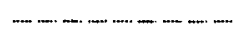 & -------- & $------m$ \\
\hline Qum. & 斗: & 675400.00 & 79222,75 & 909074.56 & 102037.62 & 1147610.12 \\
\hline
\end{tabular}

YEAF:

\section{C.Ain}

MOP:? 1

MOF? ?

FulE I

Fin $|1 \ldots|$

$F(\|)$

MATH?

$Y \mathrm{AF} \cdot 3$

Eit.

\begin{tabular}{|c|c|c|c|}
\hline \multicolumn{2}{|l|}{$Y E A$} & 1997 & $19 \%$ \\
\hline LAm & 9 & 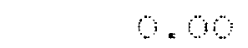 & $0 \quad 0$ \\
\hline MorT. 1 & $t$ & 0.0 & ol \\
\hline MORT. 2 & t: & 0.00 & 0.00 \\
\hline FUEL 1 & $\$$ & $115215 \times 41$ & 116176.42 \\
\hline FUEL 2 & \$ & 0.00 & 0.90 \\
\hline FUEL $\Xi$ & * & 0.00 & 0.00 \\
\hline WAINT. & 韦 & 9196.5 & 9119.81 \\
\hline & & $--m-\cdots$ & 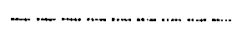 \\
\hline YEAF $S$ & $\$$ & 124411.92 & $122896-23$ \\
\hline & & $--\infty-1-1$ & 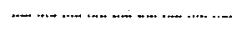 \\
\hline & & $--\ldots-\cdots$ & (.................. \\
\hline
\end{tabular}

Cutr.

\begin{abstract}
199
\end{abstract}

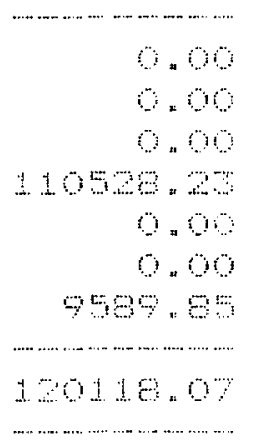

…………

क $1277 \%$

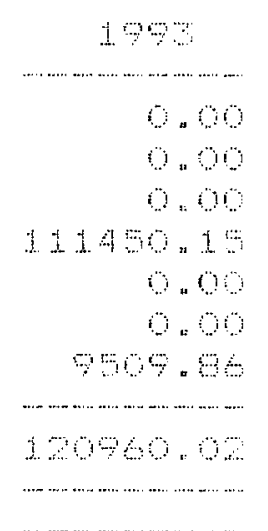

10 बक

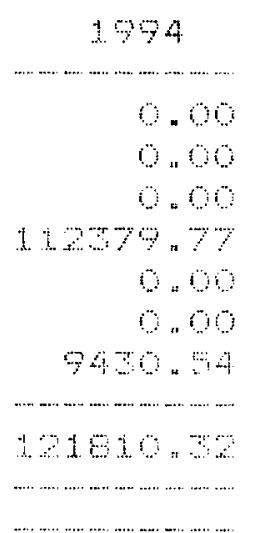

15 10100,0

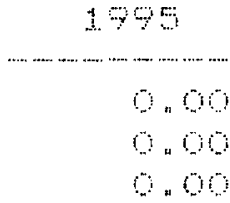

11337.14

0.0

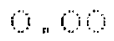

9.4 .80

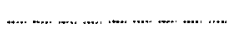

$+22669,92$

……………................

ㄱ... …….........................

162976

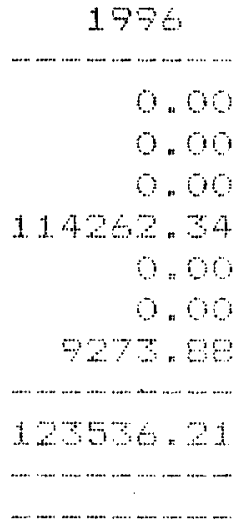

175011.6

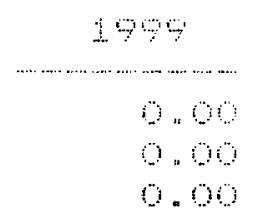

117145,45

0.0

0.00

$904: 74$

$12610 \%$.

…… - . - .......................

……...............................

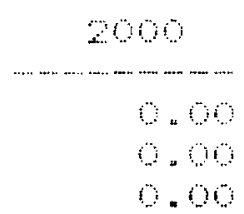

118122 - 58

\%. 0

0.00

9969.51

12709.89

…………..............

…………........... …

225970.20
201

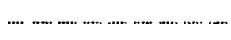

a

in

0.6

119107.85

o. 0

0.00

889.50

12001.36

-... - - - -...- - -

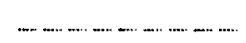
238701.50 


\begin{tabular}{|c|c|c|c|c|c|c|}
\hline \multicolumn{2}{|c|}{ YEAE } & 200 & धण & 2004 & 200 & 200 \\
\hline $\mathrm{CAOH}$ & s & 0.0 & 0.6 & 0.0 & 0.00 & 0.00 \\
\hline MOFT .1 & $t$ & . & " लि & ओ बो & 0.00 & 0.00 \\
\hline Fom, & is & ओ & ओ & ॠण & 0.00 & $\mathrm{oo}$ \\
\hline FUE: 1 & 管 & 120101,3 & A2țతat & 122113,24 & 12313190 & 124150.84 \\
\hline FUEL & $\%$ & 0.0 & ब. & $\mathrm{s}_{n} 00$ & o & 0.0 \\
\hline FUE & t & . & o & ? & 0.0 & ओo \\
\hline MAINT" & $\dot{\%}$ & 8919.2 & $674: 75$ & 8672,41 & 6600.47 & 8520.74 \\
\hline$\because F A F$ & 棒 & 1909206 & 49 & 17079,06 & 19179297 & $1=260758$ \\
\hline 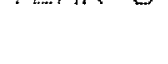 & t & $\begin{array}{l}1-0 \\
-1\end{array}$ & 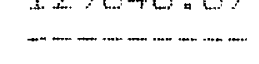 & $\frac{1}{-1}$ & $\begin{array}{l}1-1.6 \\
-\end{array}$ & $\begin{array}{l}1-200 \\
-0-10-0\end{array}$ \\
\hline & & 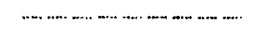 & - & 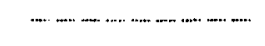 & 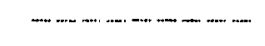 & $-\cdots-\cdots$ \\
\hline Uum. & 尝 & 25.16622 .20 & 2646471.00 & 2777257.00 & 2908999.20 & 5041676.80 \\
\hline
\end{tabular}

\begin{tabular}{|c|c|c|c|c|c|c|}
\hline \multicolumn{2}{|c|}{ YEAE } & 2007 & 200 & 2009 & 2010 & 2011 \\
\hline $\mathrm{CASH}$ & 索 & 0.00 & ${ }_{40}$ & 0.00 & 0.00 & 0.00 \\
\hline Mole? 1 & $\$$ & 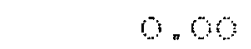 & . & 0,0 & .00 & 0.00 \\
\hline mol? & $\exists$ & o लो & ${ }_{n} 00$ & 0.0 & 0.00 & 0.0 \\
\hline FUEL. . & 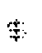 & 125194.46 & 1209.72 & . & .00 & 0.00 \\
\hline$F=10$ & s: & 0.00 & 0.00 & बो & .00 & 0.00 \\
\hline FUE & 穼 & 0.0 & . & 0.0 & .00 & " \\
\hline HATAT. & $\$$ & 845.60 & 837.05 & 0.0 & 0.0 & 0.00 \\
\hline$Y E A A=$ & $=$ & j335.05 & 134625.70 & 0.00 & \% & 0.00 \\
\hline & & ㄱ... & 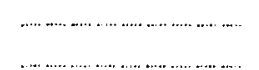 & & & \\
\hline & & 175329.90 & 9954.50 & 54.50 & 54.50 & $54 \times$ \\
\hline
\end{tabular}




\author{
FEFMILAE D-O EXFEFIMENTAL FACILITY \\ LIFE CYOLE COST ANALYIS \\ WHFIED FFEENT WOTH CUMARISUNS \\ FOF \\ MLI. SVSTENE \\ Z OCTTEEF, 1997 \\ ANALYET: ${ }_{\text {"F }}$ RESTLLOUTOH

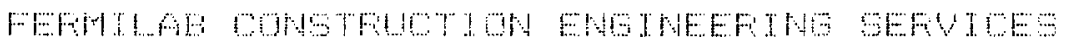




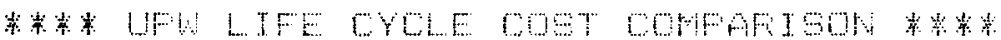
FIIE MO. : LOOI. DAT
FFOTEOT

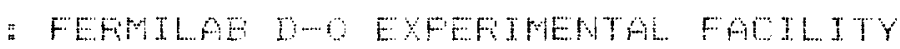
ANALYT
"S.F. IFSTLOUTH
CONMEATS
" DESTON EASTS STSTH
FILE NOW FrOTELT ANALYST DONHENTS
$\because \angle \mathrm{CO} . D A T$
- FEFMTLAE DW EXFEFTMETTAL FAOTLITY
: S.F.AFGTULOVICH
- ALTEFNATE SYSTEM NO. 1

\begin{tabular}{|c|c|c|c|c|}
\hline & (A) & (E) & $(A-B)$ & $(A-E)$ \\
\hline YEAF & FILE 1 \$ & FILE $=$ & $Y F: S$ & CuM. \$ \\
\hline 1987 & 752900.00 & 752900.00 & 0.00 & 0.00 \\
\hline 1908 & 71298.88 & 75291.50 & -3992.41 & -5972.41 \\
\hline 1999 & 71695.09 & 75720.00 & -4025.70 & -8018.12 \\
\hline 1990 & 72096.2 & $7 \in 154.52$ & -4059.30 & $-120 \% 7.41$ \\
\hline 1991 & 72502.50 & 76595.53 & $-409,16$ & -16170.56 \\
\hline 1992 & $799+54$ & 77040.64 & -4127.3 & $-2029,07$ \\
\hline 1993 & $7+29,79$ & 77491.46 & $-416 \mathrm{i}: 7 \mathrm{~d}$ & -34459.50 \\
\hline 1954 & $7 \% 71,0$ & 77947.46 & $-4+9644$ & $-286 \operatorname{al}$ \\
\hline 1995 & 74177,41 & 7840.84 & -4231 . 44 & -398.45 \\
\hline 1996 & 74608.91 & 79975.64 & $-4266=79$ & -27104.19 \\
\hline 1977 & 7504.56 & $793 \%, 90$ & $-402 \cdot 2$ & -41436 \\
\hline 1990 & 7546744 & 79525,64 & $-4-40$ & $-45794,7$ \\
\hline 1999 & 70934,5 & बणाओ : & -494 , 6 & -40169.99 \\
\hline 200 & $7-786$ "ө & $8 \% 7 \%$, 7 & -4410.67 & -3459.96 \\
\hline 2001 & 7694.47 & ச12\% - 4 & $\cdots \cdot 444 \% 67$ & $-590 \cdot 4$ \\
\hline 200 & $7790=41$ & ๑179. & $-\cdots 444,77$ & $-6012,59$ \\
\hline 200 & 7775.60 & 21\% . $\mathrm{\theta}$ & $-452,4$ & $-6004,57$ \\
\hline 2004 & 78249.3 & 8209,23 & -499.90 & -7259.47 \\
\hline 20 & $78720=40$ & 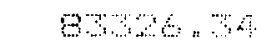 & $-459,96$ & $-77192 \times 41$ \\
\hline 200 & 79219.91 & 9649 , & $-46.96,30$ & -61928.72 \\
\hline 2007 & 7970.90 & $94,7.6$ & -46749 & -96503.69 \\
\hline 2000 & op. & 84912,3 & -4713.96 & -91217.64 \\
\hline
\end{tabular}

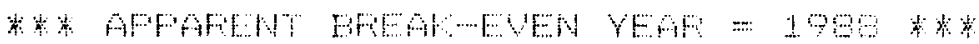




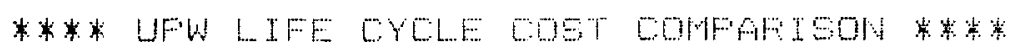

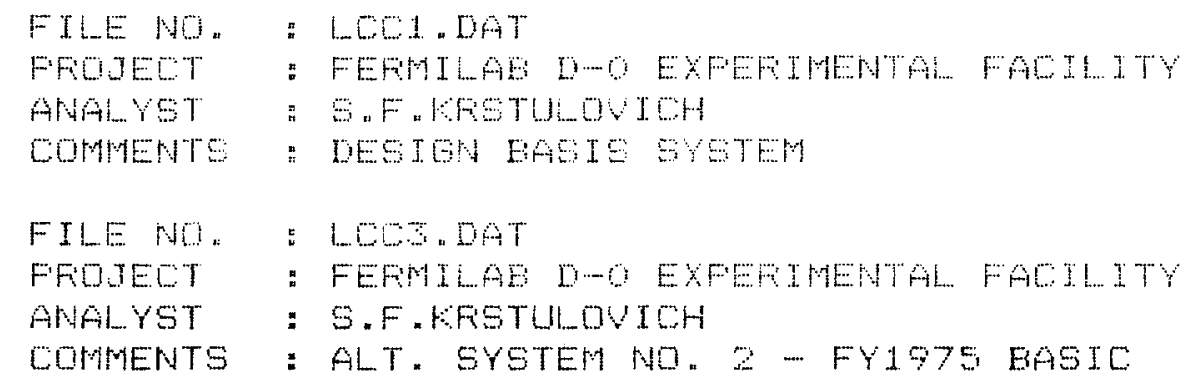

\begin{tabular}{|c|c|c|c|c|}
\hline & (A) & (E) & $(A-B)$ & $(A-E)$ \\
\hline YEAF: & FILE 1 \$ & FILE $2 \$$ & $Y F \cdot S \$$ & CUM. $\$$ \\
\hline 1907 & 752900.00 & 675400.00 & 77500.00 & 77500.00 \\
\hline 1980 & $71298.8 \mathrm{e}$ & $1168 x 2.70$ & -4553.82 & 51966.18 \\
\hline 1989 & 71695.09 & 117641.80 & -45946.70 & -1.980 .52 \\
\hline 1990 & 72096.23 & 118459.00 & -46862.77 & -60545.31 \\
\hline 1991 & 72502.90 & 119284.40 & $-46702,02$ & $-\cdots 1071$ \\
\hline 199 & 7291044 & 120110.10 & $-37204,56$ & -1543 \\
\hline $199 \%$ & $7+29,75$ & $120 \%$ ल0 & -47630 & -201960.19 \\
\hline 1994 & 7971.0 & 121810,0 & -48059.27 & -2500 \\
\hline 1996 & $7417 \% 41$ & 12269,0 & $-49491=59$ & -2965 \\
\hline 1996 & 7460.91 & $12+56.20$ & $-4892,-3$ & -247430.28 \\
\hline 1997 & 75045.56 & 12441.90 & $-49366,-6$ & -396804.62 \\
\hline 199 & 7346,44 & 1259960 & $-4960,77$ & $-446613.8 \mathrm{e}$ \\
\hline 1999 & $759-4.62$ & $12619 \%$, & -90546 & $-49686.0 \%$ \\
\hline 2000 & 7636,8 & 27का "न & $-50704,04$ & $-64752,06$ \\
\hline 2001 & 76044,47 & 12000,40 & 6.93 & $-59829,00$ \\
\hline 200 & $777 \%, 41$ & $129 \% 207$ & $-61-4,30$ & -650425 \\
\hline को & 77775 « & 129840.90 & -6207 & $-702415 \times 44$ \\
\hline 2004 & $79-49,6$ & 18976.10 & $-52-4,77$ & $-754952,25$ \\
\hline 200 & $7 \mathrm{a} / 4 \mathrm{a}$ & $10179, \infty$ & 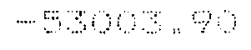 & -907966.19 \\
\hline $200 \%$ & $792+9$ & $18 \mathrm{6 \%}, 5$ & -69474.6 & -86140.60 \\
\hline बि & 77 क्ष 9 & 1852,0 & -5849.10 & $-915300,00$ \\
\hline बोक & की 19.9 & 15462.60 & $-6427+41$ & $-9690 \%$ \\
\hline
\end{tabular}

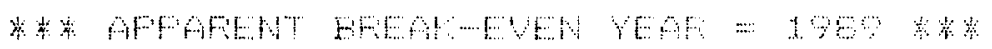




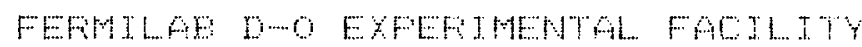 STMFE FAYAAR CALUUATTONS For
} AI $948 \mathrm{TH}$

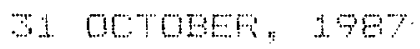

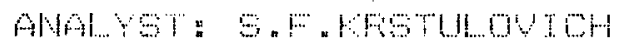

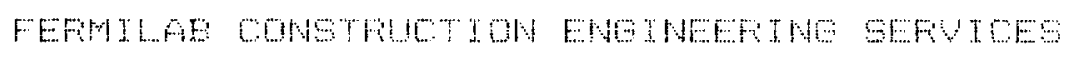


INFUT VALUES

INITIAL TOTAL COOST

ANNUAL DFEFATING COST

ANNUAL. MAINTENANCE COST

SALVAGE VALUE AT YEAF 20
DESTONED SYSTEM

$\begin{array}{rr}\$ & 752,900 \\ \$ & 59.125 \\ \$ & 12,000 \\ & \end{array}$

ALT SYSTEM NO. 1

$$
\begin{array}{rr}
\$ & 752,900 \\
\$ & 69,099 \\
\$ & 12,000 \\
\$ & 0
\end{array}
$$

DESIGNED SYSTEM

EXFENSES

YEAF: ANNUAL CUMIULATIVE
ALT SYSTEM NO. 1 EXFENSES

ANNUAL EUMULATIVE

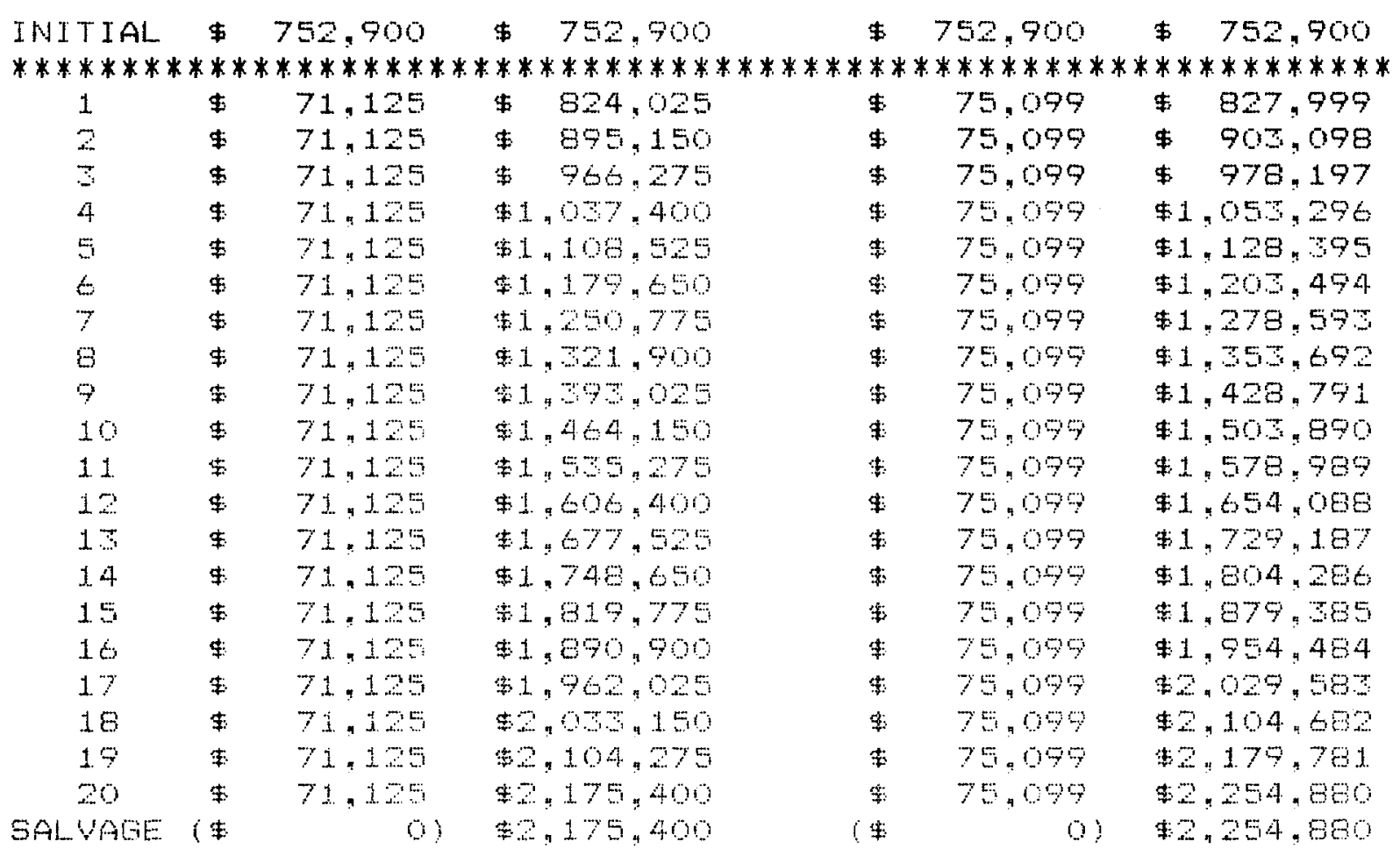

FAYEACK FOF DESIENES SYSTEM DOCUFS AFTEF O Y YEAFE ( 752,900 )

TOTAL COST OF DESIGNED SYSTEM FOF 20 YEAFS IS $2,175,400$

TOTAL COST OF AL.T SYSTEM NO. 1 FOF 20 YEAFS IS $2.254,880$ 
INUTT VALES

INTTIAL TOTAL COST

ANNUAL OFEFATINE OOST

ANUUA MATHTHANCE COST

SALVAGE VALUE AT YEAF 20
DESTGVED SYSTEM

$\begin{array}{rr}4 & 752900 \\ * & 59.120 \\ * & 12,000 \\ * & 0\end{array}$

FY1975 ALT NO. 2

$$
\begin{array}{rr}
\$ & 975.400 \\
* & 106.428 \\
* & 10.000 \\
* & 0
\end{array}
$$

FY1.775 ALT NO. 2 EXFENGES

DESIGNED SYSTEM EXFENSES

YEAE: ANRUAL CUMULATIVE

ANWUAL CUMULATTVE

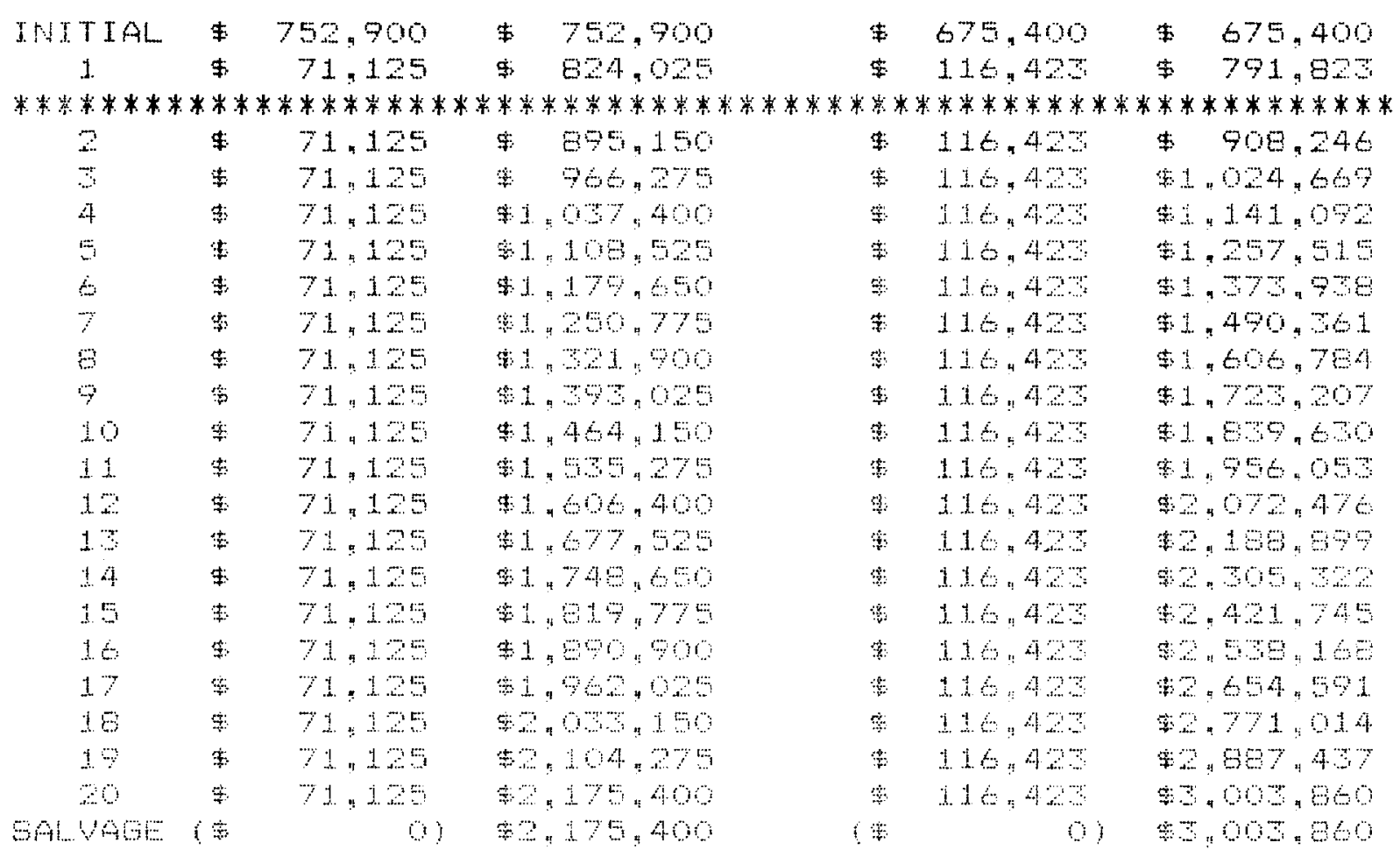

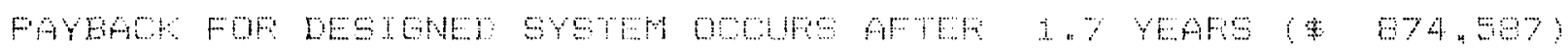

TOTAL COST OF DESTGNED SYSTEM FOF 20 YEAFS IS

TOTAL COST OF FYA7S ALT NO. 2 FOF 20 YEAFS IS $\$$. 004,860 